What is right? What is wrong?

And does the answer tell something about culture?

An investigation into culture and morality using the values concept

by

Christin-Melanie Vauclair

A Thesis

submitted to Victoria University of Wellington

in fulfilment of the requirements for the degree of

Doctor of Philosophy in Psychology

Victoria University of Wellington

2010 



\section{ABSTRACT}

Morality, or what is considered right or wrong, varies seemingly across cultures. However, the literature shows that moral psychologists have mainly investigated moral reasoning assuming a universal morality. Cross-cultural psychologists in contrast have widely neglected moral issues such as prescriptive beliefs of what people ought to do in a culture, and have predominantly measured culture through personal values. This thesis attempts to bridge this gap by focusing on the values concept. Four empirical studies were conducted to critically examine values as a measure of culture and their relationship to morality.

Chapter one is an introduction into the topics morality, culture and values. Chapter two outlines the conceptual and methodological issues associated with deriving cultural values through the statistical aggregation of individuals' personal values. A value taxonomy is presented in which personal moral values and societal moral values are proposed as alternatives for measuring the cultural context. Following this critique, personal values are examined in two empirical studies in a cross-cultural context scrutinizing the validity of Schwartz' (1994) Culture-level Value Theory. Study 1 is a cross-cultural meta-analysis using the Rokeach Value Survey (Rokeach, 1973) showing that Schwartz' culture-level value structure was replicable with different samples, and a different method for assessing value priorities. Nonetheless, a set of values not included in Schwartz' analysis formed a new value type: Self-fulfilled Connectedness (SFC) which is related to the pursuit of non-material goals and endorsed in countries in which basic needs are fulfilled. Study 2 tested in a multilevel regression model whether Schwartz' cultural values predicted individuals' moral attitudes with data from more than 40 different countries. The findings indicated that the value dimension Autonomy- 
Embeddedness explained individuals' lenient attitude towards personal-sexual, but not towards dishonest-illegal issues.

Study 3 dealt with the fundamental critique raised in chapter two that Schwartz (1994) does not operationalize micro- and macro-level constructs independently when measuring cultural values. To address this empirically, personal moral values and societal moral values were explicitly measured in student samples from eight cultures. Societal moral values showed greater cross-cultural differences than personal moral values. Furthermore, societal moral values at the culture-level conceptually replicated the multilevel findings from Study 2. This suggests that societal moral values are a valid macro-level variable for the measurement of culture. Finally, Study 4 was conducted to elicit implicit moral values. Respondents from four cultures free-listed their associations of a 'moral person'. Correspondence analyses revealed that collectivistic-oriented samples mentioned more traditional moral attributes, whereas individualistic samples reported more liberal attributes. Furthermore, accessibility of implicit moral values - matched with the SVS - correlated with explicit ratings of personal moral values from Study 3, providing convergent validity for this kind of values. This multi-method finding corroborated that personal moral values and societal moral values are different concepts.

In sum, these studies support the validity of Schwartz' theory and suggest that notions of right and wrong do indeed tell us something about culture, but it depends on (1) the issues studied (personal-sexual issues), and (2) the kind of values measured (societal moral values). 


\section{ACKNOWLEDGEMENTS}

When I decided to come to New Zealand three and a half years ago to do my $\mathrm{PhD}$ in cross-cultural psychology, I embarked upon an uncertain and exciting journey. I did not have clear expectations what it would be like. Now, being at the point of looking back, I am deeply grateful for this experience. I would like to say thank you to a number of people who turned my academic journey into a great personal experience that I will never forget.

Thanks to Ronald Fischer, my primary supervisor, for his literally unlimited enthusiasm, dedication, and guidance into the world of science. I learned a lot - first and foremost that statistics can be fun. Thanks also to Marc Wilson, my secondary supervisor, who contributed significantly to my motivation by always having something positive and encouraging to say about my work.

I deeply appreciate my colleagues from the Centre of Applied Cross-cultural Research with whom I had not only stimulating (non-/) academic discussions, but also found lifetime friends. The 'moral' support, reading of manuscripts, statistical advice, assistance with translations and data collection were invaluable. Special thanks goes to (in alphabetical order): Diana Boer, Pip Collie, Carla Crespo, Pollyane Diniz, Katja Hanke, Sammyh Khan, Magda Kielpikowski, Larissa Kus, Vivian Miu-Chi Lun, Socorro Mendoza, Astrid Podsiadlowski, Paul Reddish, Maria Strobel, Elena Stevens, Jessie Wilson.

I am also grateful to the School of Psychology and the staff for their support - in particular Ngaire Lavery who was always very helpful with a big smile on her face. A number of scholarships facilitated my research for which I am very thankful: Victoria University of Wellington PhD and submission scholarship, and grants from NZPSAA, 
School of Psychology and the Faculty of Science for funding my conference attendances and research projects.

A very big thank you goes to all the collaborators from around the world who collected data and translated my survey. Without them, a substantial part of my work would not have been possible (in alphabetical order): Prof Allan Bernardo, Prof Maria Cristina Ferreira, Dr Valeschka Guerra, Prof Klaus Helkama, Dr Ulrich Hößler, Prof Serdar Karabati, Dr Vijyendra Pandey, Prof Astrid Podsiadlowski, Prof Juliana Porto, Prof Melissa Lopez Reyes, Jennie Rytkönen, Prof Moises Kirk de Carvalho Silva, and Prof Erika Spieß. At this point I would also like to thank the numerous anonymous students filling out my questionnaire and all the research assistants helping with data entry.

Thanks to my 'PhD coaches' - Dr Carla Crespo, Dr Rachna D. Jain, and Dr Fabrice Vauclair - who helped me through the dissertation process with very wise advice.

Thanks also to my parents, Leni and Peter Fuchs. Their different cultural background (Brazil and Germany) raised my interest in cross-cultural psychology in the first place. I appreciate that they are always happy, if I am happy with what I do - even if that means that I am literally at the other end of the world. Thanks also to my little brother Christian Fuchs for his steady encouragement and for doing some of the data entry.

A deep sense of appreciation goes to my husband Fabrice for his unconditional love, his understanding, encouragement and belief in me and for supporting me wherever possible. He is my source of energy.

This thesis represents to me more than an academic accomplishment - it has been a great chapter in my life. Thanks to all of you who shared it with me. 


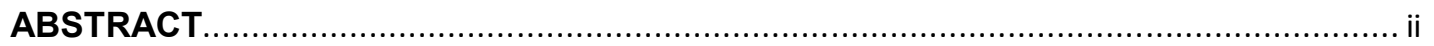

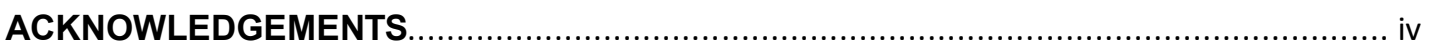

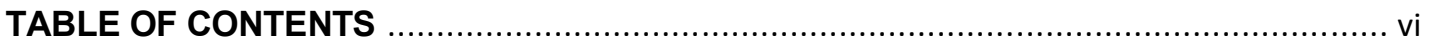

LIST OF TABLES

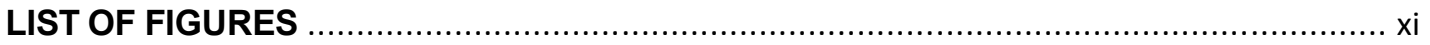

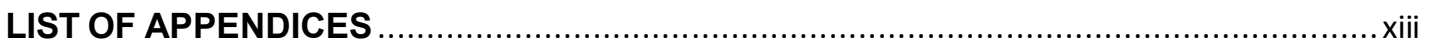

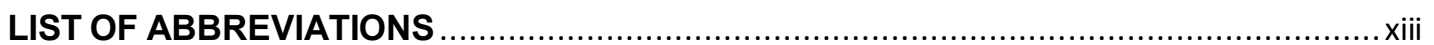

PREFACE

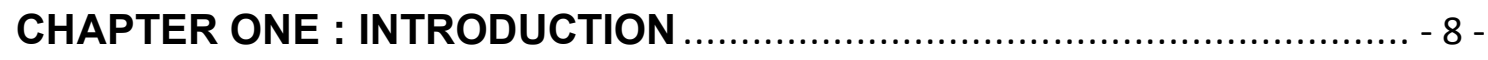

Defining Morality …............................................................................ 8 -

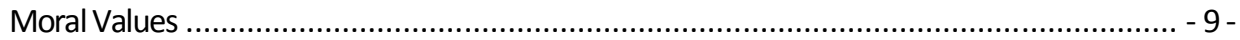

Culture and Morality................................................................................................ 12 -

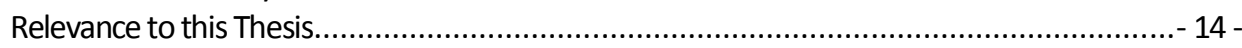

Moral Psychology ..................................................................... 16 -

The Normative Account........................................................................................... 16 -

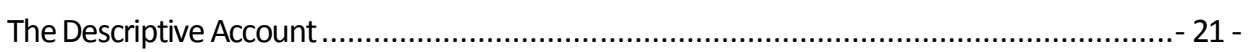

Theoretical Stance in this Thesis .................................................................................... 27 -

Cross-cultural Psychology .................................................................... 28 -

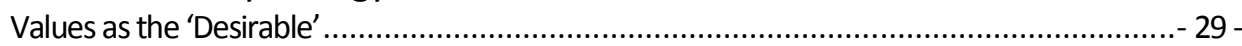

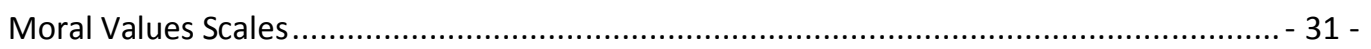

Values as Terminal and Instrumental Goals .................................................................. 34 -

Schwartz' Value Survey and Theory ...............................................................................- 37 -

The Individual-level ................................................................................................... 37 -

The Culture-level .................................................................................................. 43 -

A Note on the Ecological Fallacy................................................................................................

Conclusion.......................................................................................... 50 -

CHAPTER TWO : THEORETICAL PROPOSAL ................................. 51 -

Measuring Cultural Values at the Individual-level:

Considering Morality in Cross-cultural Value Research

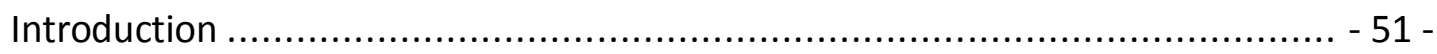

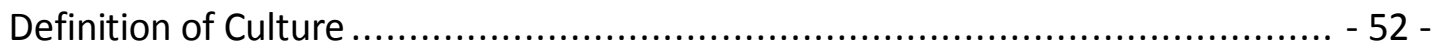

Past Approach in Identifying Cultural Values........................................ 54 -

The Culture-level Analysis Approach ............................................................................... 54 -

Conceptual and Methodological Problems...................................................................... 55 -

Empirical Evidence Underpinning the Problems................................................................ 58 -

Alternative Ways of Measuring Cultural Values........................................ - 60 -

Recent Approaches: Changing the Frame of Reference......................................................- 60 -

Classifying Value Research at the Individual-level: A Value Taxonomy ....................................- 62 Incorporating Morality in the Measurement of Values .................................................... 63 -

Benefits of Distinguishing Different Value Measurements ............................................... 67 -

Conclusion and Final Remarks.................................................... 70 - 
The Structure of Human Values at the Culture-level:

A Meta-analytical Replication of Schwartz' Value Orientations using the RVS

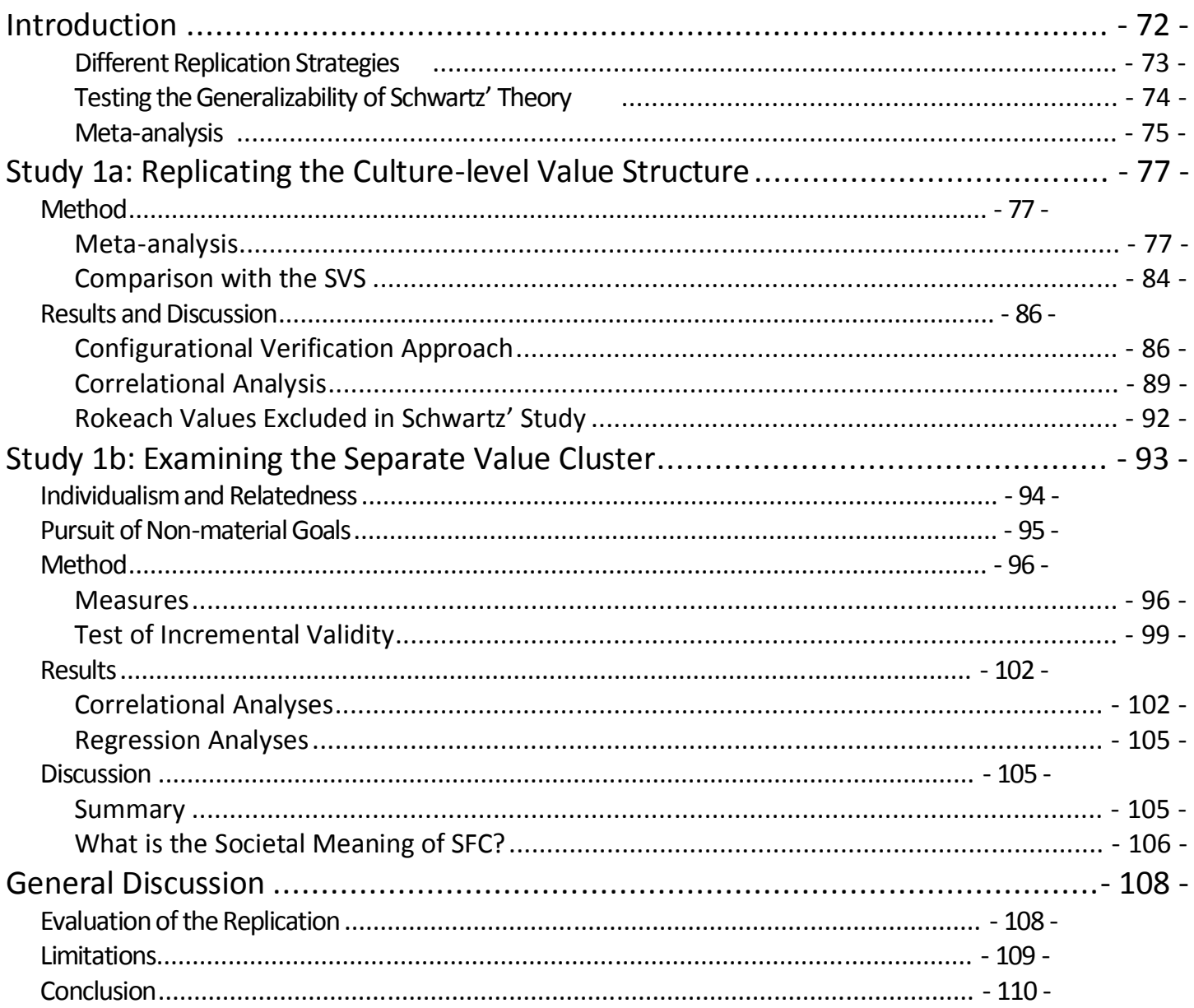

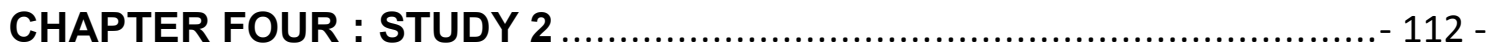

Do Cultural Values Explain Individuals' Moral Attitudes?

A Cross-cultural Multi-level Approach

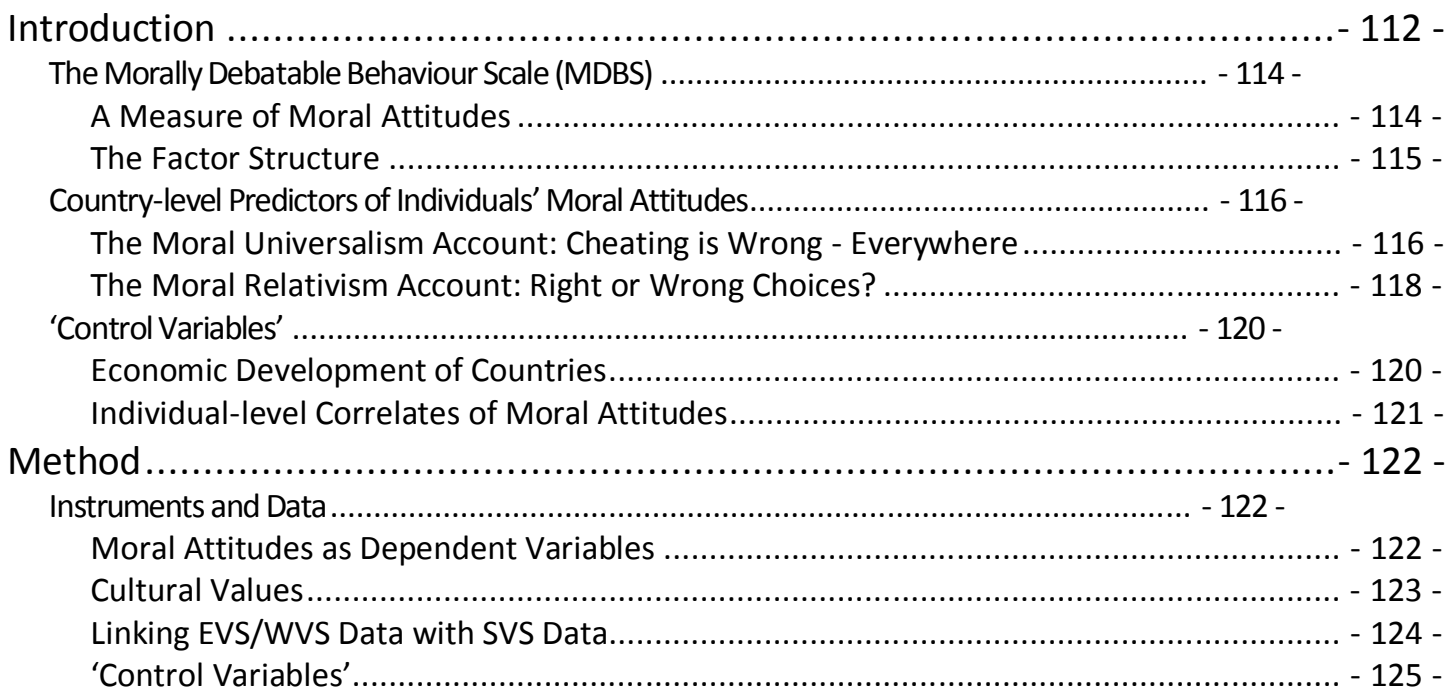


Analytical Strategy

Construct Equivalence of Moral Attitudes

Multilevel Regression Analysis ........................................................................... 127 -

Results

Factor Structure of the MDBS

$129-$

Prediction of Moral Attitudes

132

Attitudes towards Dishonest-illegal Behaviours......................................................... - 132 -

Attitudes towards Personal-sexual Behaviours................................................... 139 -

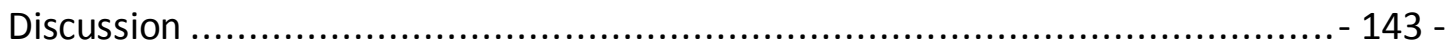

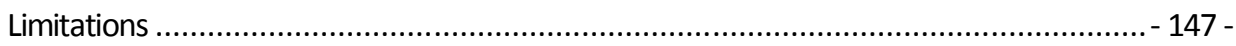

Conclusion .............................................................................................. 149 -

CHAPTER FIVE : STUDY 3

Identifying Cultural Differences and Similarities through the

Explicit Measurement of Moral Values

Introduction

An Alternative Value Measure

- $152-$

Which Values May Be Moral Values? Examining Value Hierarchies

- 154 -

Cultural Values and Individuals' Moral Attitudes............................................................. 157 -

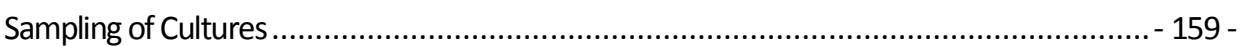

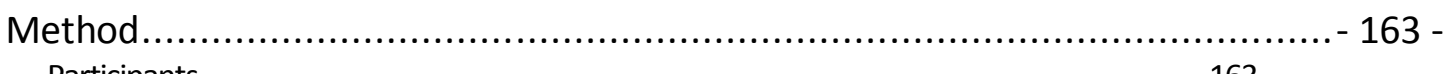

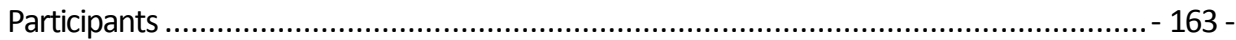

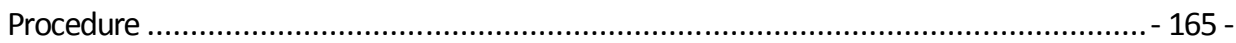

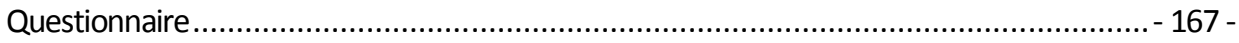

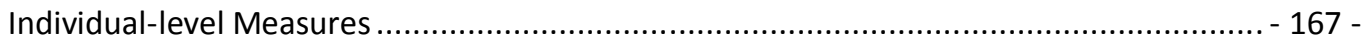

Translation ..................................................................................................... 170 -

Culture-level Value Measures ......................................................................... 170 -

Analytical Strategy ............................................................................................ 171 -

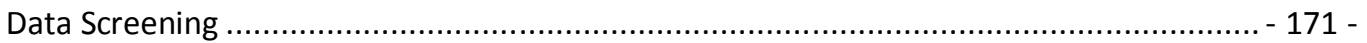

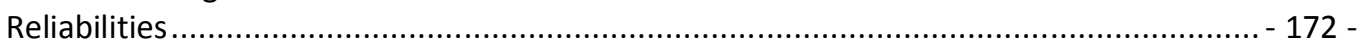

Analysis of Value Type Hierarchies ..................................................................... 174 -

Multilevel Regression Analysis ....................................................................... 175 -

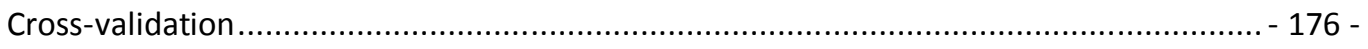

Results and Discussion ..................................................... 177 -

Confirmatory Factor Analysis on Moral Attitudes................................................. - 177 -

Reliabilities of Value Measures ........................................................................ 178 -

Differences and Similarities of Response Scales and Cultural Groups .................................. 180 -

The Pan-cultural Value Type Hierarchy............................................................ - 180 -

Culture-specific Value Type Hierarchies .................................................................. 181 -

Multilevel Regression Analysis ........................................................................ - 187 -

Follow-up Analyses: Individual-level Mediation Model .............................................. 193 -

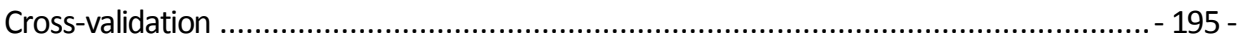

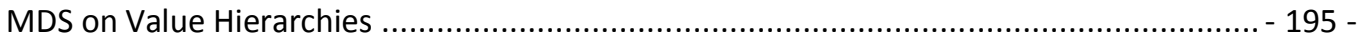

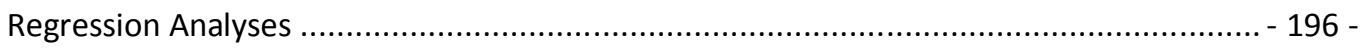

Individual-level Mediation Model .............................................................................. 198 -

General Discussion .......................................................... 199 -

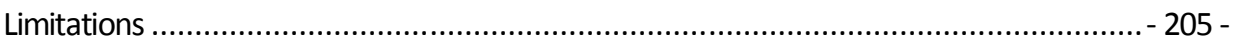

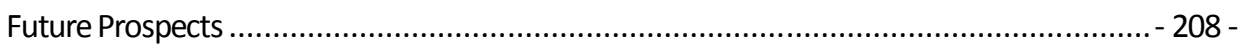


An Implicit Measure of Moral Values:

Laypeople's Associations of the Moral Person

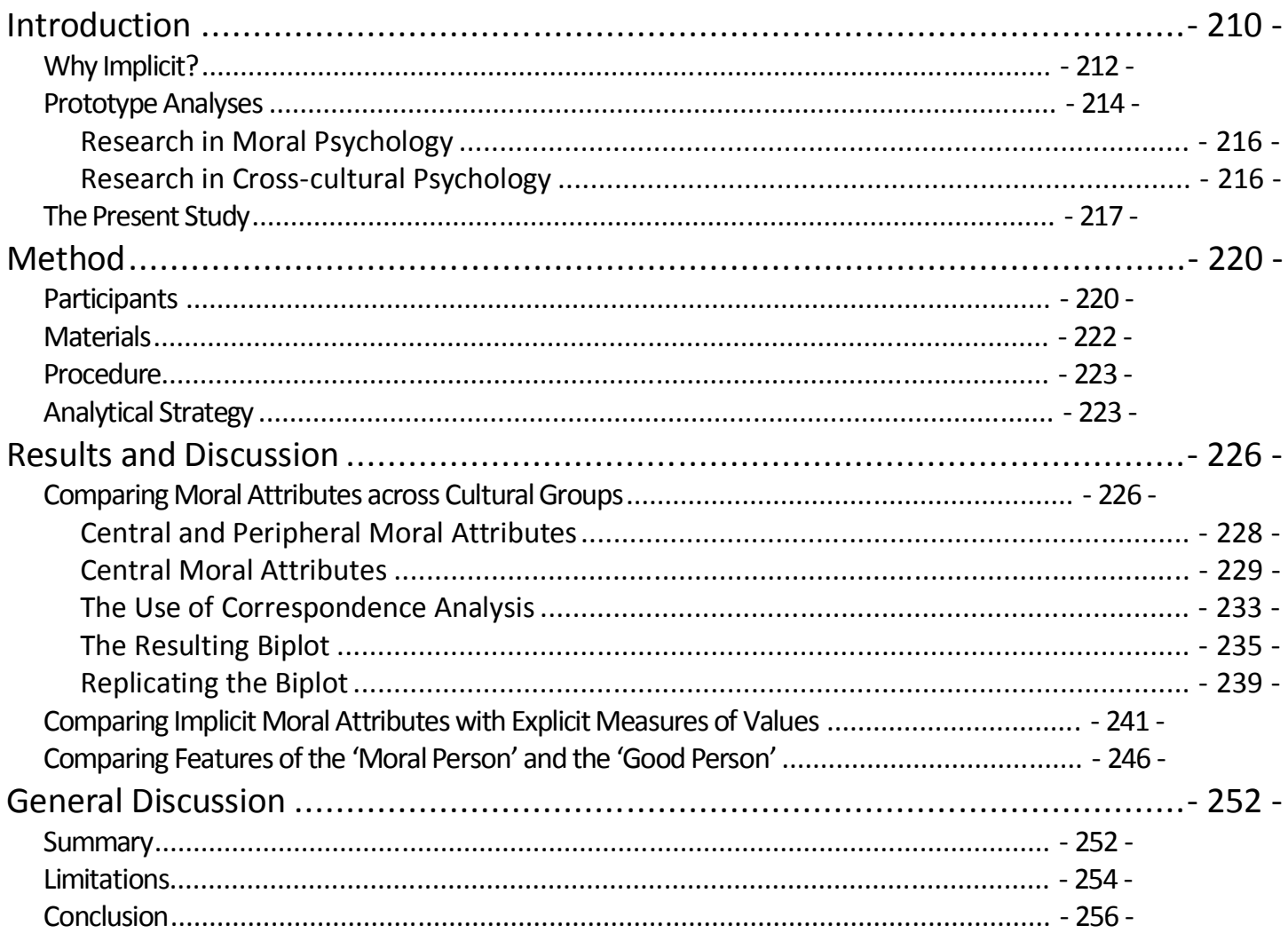

CHAPTER SEVEN : GENERAL DISCUSSION ................................ 260 -

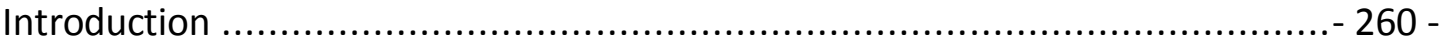

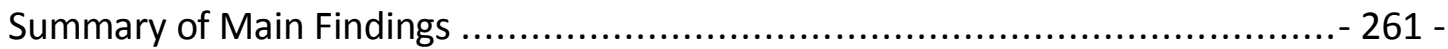

Strengths of this Research ............................................................... 268 -

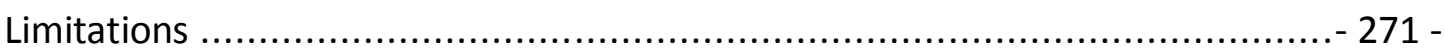

Contribution to the Literature ............................................................. 274 -

Cross-cultural Psychology.................................................................................... 274 -

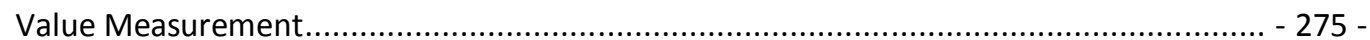

Individualism-Collectivism and Values ................................................................ 277 -

Individualism-Collectivism and Morality .............................................................. $278-$

Beyond Individualism-Collectivism? ............................................................... 279 -

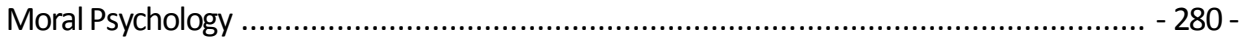

Investigating Morality from a Descriptive Approach........................................ - $280-$

Moral Values............................................................................................ - $282-$

Suggestions for Future Research..................................................................... - 286 -

Practical Implications ....................................................................... 289 -

Final Conclusion ..................................................................... 290 -

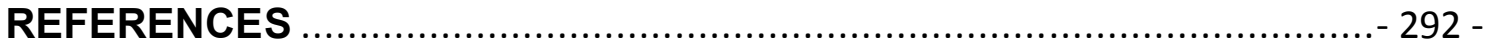

APPENDICES ........................................................................... 311 - 


\section{LIST OF TABLES}

Table 1.1: Value types, their definitions and cross-culturally equivalent value items that represent them. $-41-$

Table 2.1: Value taxonomy: The measurement of values at the individual-level ..... - 63 -

Table 3.1: Comparison of Rokeach and Schwartz' values.................................... - 78 -

Table 3.2: Sample characteristics of the meta-analysis per country. $-81-$

Table 3.3: Spearman correlations between Schwartz' and Rokeach culture-level value structure based on MDS. - 89 -

Table 3.4: Relative ranking of SFC and socio-economic indicators of 32 countries. $-100-$

Table 4.1: PCA factor loadings of the MDBS derived from the pooled sample......- 130 -

Table 4.2: Multilevel regression analyses for attitudes towards dishonest-illegal issues. $-133-$

Table 4.3: Multilevel regression analyses for attitudes towards personal-sexual issues. $-141-$

Table 5.1: Sample characteristics of Study 3. $-164-$

Table 5.2: Values included in Version 1 and Version 2 of the Moral Values Survey. $-166-$

Table 5.3: Individual-level mean item-intercorrelation (mean $r_{i j}$ ) for each sample and response scale averaged across value types. - 179 -

Table 5.4: Individual-level Cronbach alphas for each sample and response scale averaged across value orientations. 179

Table 5.5: Pearson correlations of predictors and criterion variable. $189-$

Table 5.6: Summary of hierarchical regression analysis predicting individuals' strictness in their attitudes towards personal-sexual issues (Survey Version 1, Model 1). $191-$

Table 5.7: Summary of hierarchical regression analysis predicting individuals' strictness in their attitudes towards personal-sexual issues (Survey Version 1, Model 2). $192-$

Table 5.8: Summary of cross-validating hierarchical regression model predicting individuals' strictness in their attitudes towards personal-sexual issues (Survey Version 2, Model 1). $197-$ 
Table 5.9: Summary of cross-validating hierarchical regression model predicting individuals' strictness in their attitudes towards personal-sexual issues (Survey Version 2, Model 2). .............................................................. - 198 -

Table 6.1: Sample characteristics of Study 4. ..................................................... - 222 -

Table 6.2: Attributes of a moral person in four cultures....................................... 227 -

Table 6.3: Correlations between percentages of free-listed moral features across four cultures. ............................................................................... 229 -

Table 6.4: Correlations between percentages of free-listed 'central' moral features across four cultures. $-231-$

Table 6.5: Adjusted frequencies of matches between moral trait categories and Schwartz'value types. $-242-$

Table 6.6: SVS dimensions correlated with frequencies of moral trait categories. - 244 -

Table 6.7: Spearman correlations between implicit and explicit measures of values. $-246-$

Table 6.8: Attributes of a 'good' and a 'moral' person free-listed by two New Zealand samples. $-248-$

Table 6.9: Attributes of a 'good' and a 'moral' person in two Filipino samples.... 249 _

\section{LIST OF FIGURES}

Figure 0.1: Schematic overview of purpose and main characteristics of the empirical studies conducted within this thesis

Figure 1.1: Circular model of relations among ten motivational value types at the individual-level (adapted from Schwartz, 2006b, p. 965;

Schwartz \& Sagiv, 1995, p. 96).

Figure 1.2: Circular model of relations among seven value dimensions at the culture-level (from Schwartz, 2006, p. 142). $-44-$

Figure 3.1: MDS representation of all 36 Rokeach values $-88-$

Figure 3.2: Spearman correlations between average ranking of SFC and Schwartz' culture-level value types. $-103-$

Figure 4.1: Hypothesized multilevel model for the prediction of individuals' moral attitudes. 
Figure 4.2: Cross-level interactions for attitudes towards dishonest-illegal and personal-sexual issues based on teachers' cultural values

Figure 5.1: Co-plot map of 67 national groups on the value dimension AutonomyEmbeddedness (adapted from Schwartz, 2006). $162-$

Figure 5.2: Example of bipolar rating scales measuring five different evaluations of values. 168

Figure 5.3: One-dimensional MDS representation of value hierarchies using five different response scales (pan-cultural sample, Version 1 and 2 of the Survey).

Figure 5.4: Two-dimensional MDS representation of response scales and cultural samples. $182-$

Figure 5.5: Mean ratings across ten value types displaying acquiescence tendencies in the collectivistic sample.

Figure 5.6: Value ranking profile of individualistic and collectivistic samples for societal moral values (societal expectation ratings) and personal moral values (moral value ratings).

Figure 5.7: Two mediation models for different value ratings at the individual-level. - 194 -

Figure 6.1: Judgment rules for the content analysis of moral attributes. 224 -

Figure 6.2: Examples of frequency scree plots of free-listed moral attributes in two samples ..... $232-$

Figure 6.3: Biplot of relative frequencies of moral trait categories across four cultural samples. $-237-$

Figure 6.4: Two-dimensional representation of correspondence analysis results for frequencies of 'moral character' and 'good character' trait categories across samples 250 -

Figure 7.1: Schematic overview of main findings and linkages between studies. ..- 262 - 


\section{LIST OF APPENDICES}

APPENDIX A: List of references included in the meta-analysis $-311-$

APPENDIX B: Figure illustrating the distribution of countries regarding their socio-economic development and importance of SFC values..... $315-$

APPENDIX C: Cross-cultural Moral Values Survey (Version 1) $-316-$

APPENDIX D: Value type rankings under five response scales in four individualistic and four collectivistic samples (Version 1)....

APPENDIX E: Comparison of MDS representations based on rankings (left figure) and mean ratings (right figure) after transformation to maximal similarity with GPA

APPENDIX F: Example of free-listed moral traits categories

(Filipino responses)

APPENDIX G: Biplots using correspondence analysis based on culturally idiosyncratic moral trait categories (left figure) and all 49 moral trait categories (right figure)

APPENDIX H: Moral trait categories matched to values of the Schwartz' Value Survey $-343-$

\section{LIST OF ABBREVIATIONS}

CLOP Cross-level Operator (a form of multilevel modelling)

$\boldsymbol{E} \boldsymbol{V S} / \boldsymbol{W} \boldsymbol{V} \boldsymbol{S}$ European/ World Value Survey

EXP Scale assessing societal moral values through expectation ratings

GPA Generalized Procrustes Analysis

HLM Hierarchical Linear Modelling

IMP Scale assessing personal values through importance ratings

MDBS Morally Debatable Behaviour Scale

MDS Multidimensional Scaling

MOR Scale assessing personal moral values through moral value ratings

$\boldsymbol{R} \boldsymbol{V} \boldsymbol{S} \quad$ Rokeach Value Survey

SFC Self-fulfilled Connectedness

SVS Schwartz' Value Survey 



\section{PREFACE}

This thesis is a critical examination of the cultural values concept employed in crosscultural research. Cultural values are usually measured through the aggregation of personal values at the culture-level. The study of moral values is here proposed as a valid alternative for identifying cultural differences and similarities.

The motivation to study moral values across cultures was stimulated by my Master thesis research (Fuchs, 2004) which dealt with cultural differences at the workplace experienced by German expatriates in Brazil (see also Brökelmann, Fuchs, Kammhuber, \& Thomas, 2005; Fuchs, 2005, 2006). I used the critical incident technique (Flanagan, 1954), refined and adapted to the investigation of intercultural encounters for German sojourners by my supervisor Prof. Alexander Thomas (see, e.g., Thomas, 1996). Critical incidents in the intercultural context are surprising or even upsetting events for an individual that occur because a person behaved in a way that was not expected. One of the take-home messages from this research for me was that people do have clear culturally-based expectations about how things 'ought to be' or 'ought to be done'. There are culturally different conceptions of what is right and acceptable which if violated can lead to very upsetting experiences in intercultural encounters. Hence, the idea emerged that identifying the more general standards that guide people in their sense of right and wrong - i.e. their moral values - should provide valuable insights into the understanding of cultural differences and similarities in behaviour. This thought was further nourished by a controversy and critique in cross-cultural psychology, directed at the common practice to derive culture from a descriptive (i.e. statistical) norm by taking the average of what a group of people personally want in their lives instead of measuring the external constraints or the deontological and ideological norm (see Gelfand, Nishii, \& Raver, 2006; cf. Hofstede, 1998; Moghaddam \& Studer, 1997). 
Besides intercultural encounters, the concept of moral values also plays an important role in other areas of societal relevance. In the political arena, for instance, moral values have been regarded as people's motivation to vote for President Bush in the 2004 presidential election in the United States (Skitka \& Bauman, 2008). Wright, Cullum and Schwab (2008) pointed out that much political and social unrest involves discrepancies in what people believe to be a moral value or not. Skitka and Mullen (2002) demonstrated this with a real-life example: the custody battle involving Elián González, a Cuban boy whose mother died when they fled from Cuba. The case aroused enormous public interest and attention, and people from the USA and Cuba were divided about the right course of action. The authors argue that the violation of moral values can motivate people to publicly defend them and can even lead to civil unrest which may range from peaceful demonstrations to violent protests and even turn into terrorism and revolution (see also Skitka, 2002). This is also applicable to the recent conflict concerning the protests in the Islamic world after the publication of the caricatures of Mohammed by a Danish cartoonist. Heine and Norenzayan (2006) argued that the protests are a result of different conceptions of right and wrong. In this case it may have been a clash between the value of freedom to express one's opinion in the Western world and the value of sacredness in the Islamic world. Though one might also argue that political reasons played an important role inciting the protests. Nonetheless, it was still a moral issue in the first place that motivated ordinary people to participate in demonstrations and that may have then been exploited for political reasons.

The great importance of moral values for social interactions lies in the fact that what is believed to be moral is psychologically experienced as objectively grounded and non-negotiable (Skitka \& Mullen, 2002). The intrinsic characteristic of moral beliefs as something non-negotiable renders divergent opinions especially difficult to deal with, since every side is convinced of knowing what is morally right or wrong. For example, 
in the case of political liberals and conservatives who differ fundamentally in their moral outlook and values (Graham, Haidt, \& Nosek, 2009). This makes it especially hard to find common ground in moral dialogues. Recent research has also shown that simply believing an issue to be 'moral' results in greater intolerance and distancing from people with divergent beliefs (Skitka, Bauman, \& Sargis, 2005; Wright, et al., 2008).

But what exactly is behind the concept of 'moral values'? What is the exact content of the moral values people adhere to, which seems to contain so much relevance and potential to mobilize people? Is there indeed a strong division in terms of the moral values people endorse in different cultures? Interestingly, there is not much research on moral values per se, i.e. on values operationalized as instrumental or terminal goals (Rokeach, 1973) and which may be believed to be morally relevant in a certain culture. Instead, moral values are often researched by focusing on attitudes towards transgressions or immoral behaviour. Hence, moral values are investigated in a form in which there is actually a lack of moral values. By reviewing the literature, one is left with the impression that it is a somewhat elusive concept. The difficulty in researching moral values may also lie in the fact that the term combines two distinct research traditions (Eckensberger, personal communication, August 28, 2007): research in moral psychology, which so far has not paid much attention to values, and research in social and cross-cultural psychology which has not focused on morality in the measurement of values. The vast majority of values research in cross-cultural psychology has dealt with personal values. However, values may be expressed in ways beyond personal importance and in ways that have important consequences for the assessment of culture.

A number of cultural psychologists have, for example, pointed out that morality and culture are inextricably linked to each other (Eckensberger, 2007; Miller, 2001) so that identifying the moral values of a culture goes hand in hand with identifying its 
cultural values. Nevertheless, there is a lack of research regarding the assessment of moral values in different cultures. This is also evident by inspecting the publication output of the two most prominent journals for cross-cultural studies: the Journal of Cross-cultural Psychology (JCCP) and Culture \& Psychology (C\&P). If "moral values" are used as a keyword in publication titles, there are zero hits for both journals. The term "moral*" yields 22 hits for JCCP and 3 for $C \& P$ which corresponds to $1.3 \%$ and $0.6 \%$ of all publications in the two journals. Most of the publications in JCCP $(81.1 \%)$ and all of the papers in $C \& P$ referring to "moral" in their title concern moral reasoning and/ or moral development. A similar search in the Journal of Moral Education (JME) using "cultur*" as a keyword in publication titles yields 20 hits and shows that the majority of these articles $(80 \%)$ are theoretical contributions or empirical papers dealing primarily with moral reasoning and moral development. Although the key term "moral values" in publication titles yields seven relevant hits in $J M E$, two of these articles are again theoretical papers and the remaining five are empirical studies measuring moral values through immoral behaviours, religious commitment or value inventories that assess the personal importance of values rather than moral values per se.

Hence, there is a lack of research on moral values across cultures. I intend to address this lack in my thesis by pursuing two goals. The first of these is a critical examination of the cultural values concept in the way it is commonly employed in cross-cultural research using the Schwartz' Value Survey (SVS, Schwartz, 1992) as an example. Schwartz' survey and the model he developed on the dynamic relations among value types, is widely regarded as the most important recent development in research on values. The second goal is to propose an alternative assessment of values that focuses on evaluations of what is morally relevant at the personal and societal-level, and to test this theoretical proposal empirically. The following paragraphs provide a brief outline of the chapters in this thesis. 
Chapter one is an introduction to the link between culture and morality, followed by a summary of the different theoretical frameworks, and their definitions of morality in moral psychology. In the process, I will elaborate on my own theoretical position. Cross-cultural value definitions and research are then critically reviewed in light of their link to morality. The Schwartz' Value Theory and survey is finally described.

Chapter two specifically scrutinizes the common practice in cross-cultural research of measuring culture through the aggregation of personal values. I take the Schwartz' Value Survey as an example and highlight conceptual and methodological issues associated with this practice. I propose to study moral values as an alternative for the measurement of culture and finish with making suggestions of how this could be implemented.

Chapters three to six describe the empirical studies in this thesis. A schematic overview of the purpose and main characteristics of the four empirical studies is provided in Figure 0.1. All four studies have a cross-cultural focus and comprise both individualistic and collectivistic samples, as identified in prior research (Hofstede, 1980; Schwartz, 1994b). The data are based on both primary and secondary sources and analyzed with a variety of statistical techniques including multidimensional scaling (Kruskal \& Wish, 1978), multi-level regression analysis (Raudenbush \& Bryk, 2002) and prototype analysis (Fehr \& Russell, 1984).

Study 1 in chapter three assesses the generalizability of Schwartz' (1994) Cultural Values Theory using different samples and a different value survey. The Rokeach Value Survey (RVS, Rokeach, 1968, 1973) was utilized for this purpose. Data from 37 cultural samples were gathered by conducting a meta-analysis on the RVS. The underlying culture-level value structure of the RVS was examined and compared to Schwartz' $(1994,2006)$ culture-level value structure. 
The critical examination of Schwartz' cultural values continues in chapter four by testing their predictive validity in the moral realm. Study 2 examines whether cultural values account for individuals' moral attitudes. A multilevel regression model was used with Schwartz' cultural values as the higher level independent variables and data from the European/ World Value Survey (EVS/ WVS) on moral attitudes as the lower level dependent variables.

Chapter five (Study 3) describes an empirical test of the theoretical proposal suggesting that moral values may be an indicator of culture (see chapter two). A survey was constructed using the Schwartz' Value Survey with modified response scales in order to measure moral values. Similarly to the previous study, a multilevel regression analysis was conducted to examine whether moral values at the aggregated level predicted moral attitudes at the individual-level.

Study 4 in chapter six was aimed at deriving moral values using a qualitative method. Individuals were asked about attributes they associate with a 'moral person'. The open-ended responses were examined using prototype and correspondence analysis. The resulting moral trait categories were also matched with values from the SVS and compared to the findings from Study 3.

Finally, chapter seven summarizes the main findings and their contribution to the literature. The strengths and limitations of the studies are discussed as well as some ideas on future studies and practical implications of this research. 


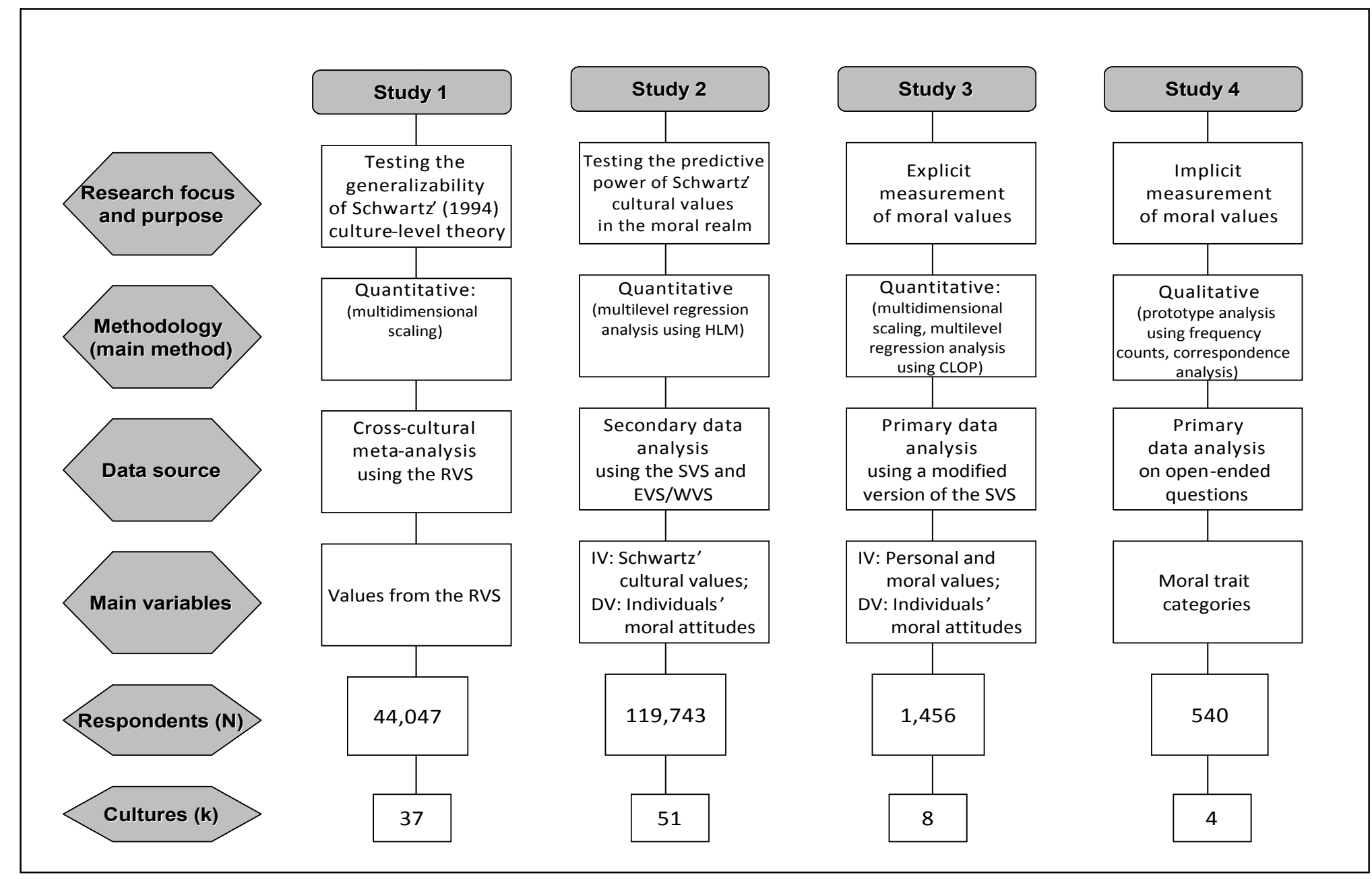

Figure 0.1: Schematic overview of purpose and main characteristics of the empirical studies conducted within this thesis.

Note. RVS = Rokeach Value Survey, SVS = Schwartz' Value Survey, EVS/ WVS = European/ World Value Survey, HLM = Hierarchical Linear Modelling, CLOP = Crosslevel Operator. 


\section{Defining Morality}

The moral sense is so ubiquitous that its lack is regarded as a pathological condition [...].

- Diane Sunar (2002)

The term morality is quite abstract for something that is central in our everyday social life. Morality facilitates group functioning (de Waal, 1996; Haidt \& Joseph, 2004; Krebs, 2008) and makes group living easier by regulating personal behaviours that reflect self-interest and self-indulgence (Janoff-Bulman, Sheikh, \& Hepp, 2009). A shared morality is vital for the functioning of any society (Bandura, 1991). Many behaviours are personally advantageous, but detrimental to others or violate their rights. Without some consensus in moral codes, people would disregard other's rights and welfare whenever their desires come into social conflict.

Despite agreement in the research community on the social psychological importance of morality, there is considerable disagreement on what exactly is morality. Definitions of morality vary depending on the underlying theoretical framework the researcher adopts (e.g. Shweder, Much, Mahapatra, \& Park, 1997; Turiel, 1983), so some of these frameworks will be briefly discussed below. What is common to the different perspectives on morality is that morality refers to notions of right and wrong. It concerns often actions and behaviours with an ought or should quality. When it refers to a 'code of conduct', the focus is usually on what a person should not do as, for example, in the moral rule 'You should not steal'. This focus on negative outcomes has been called 'proscriptive' morality (cf., for example, Janoff-Bulman, et al., 2009). 
An immoral person is one who has transgressed a rule and failed to inhibit a certain behaviour (for instance, not to steal).

Besides this mandatory and transgression-focused morality, there is also a 'prescriptive' morality which is more abstract and sensitive to positive outcomes and hence focused on what one should do. This kind of morality often refers to values like, for example, the value 'to be helpful'. A prescriptive immorality is an omission, i.e. a failure to activate a certain behaviour when, for example, not helping a person in need. Hence, a prescriptive morality has been ascribed to involve a motivational activation or an "approach-based motivational system" (Janoff-Bulman, et al., 2009, p. 533). It provides a more general than specific guidance of behaviour as is the case with proscriptive morals.

\section{Moral Values}

A prescriptive morality is sensitive to positive outcomes and refers as such to moral values (e.g., to help others, see Janoff-Bulman, et al., 2009). Since Rokeach's (1973) seminal research, values are commonly conceptualized in terms of instrumental values (i.e. modes of conduct, e.g. to be helpful) and terminal values (i.e. end-goals, e.g. equality). Moral values can be regarded as the modes of conduct or end-goals that are the "normatively prescriptive constructs by which we evaluate and regulate our social actions and shared lives" (Vine, 1983, p. 23). They are expectations defined for oneself and for others about how people should live their lives, serving as yardsticks against which people assess the social propriety of behaviours or outcomes (cf. Henry \& Reyna, 2007). Moral values can be similar to virtues, but they are not identical with them (Peterson \& Seligman, 2004). Virtues are commonly conceptualized as character strengths measured through traits and habitual actions (e.g. gratitude, persistence). Hence, virtues contain a narrower focus on morality (Graham, et al., 2009), whereas 
moral values are the broader concept including also values that are not character strengths, such as the value of social justice.

Moral values are mostly a group-level phenomenon that requires shared agreement, and refers to the individual's knowledge and internalization of moral norms and conventions. However, moral values exist also at the individual-level. A personal moral value can be defined as "a particular individual's concept of an ideal state of affairs or relations among people which he uses to assess the 'goodness' or 'badness'; the 'rightness' or 'wrongness' of [what] he observes" (Scott, 1959, p. 301). Personal moral values can be consistent with the moral values of the wider society. However, in individual cases personal moral values can also be distinct from the shared moral values of the collective, because society is not the only source of moral value education. Parents, peers, other adults and other symbolic models play an influential role, too, and can stimulate the development of differential moral values (cf. Bandura, 1991).

Another group-level phenomenon that is closely related to moral values is the concept of social norms. Social norms are expectations of "how people are supposed to act, think, or feel in specific situations" (Popenoe, 1983, p. 598). Much research on morality is focused on, or formulated around, the transgression of social norms (e.g. Turiel, 1983). Norms can be distinguished into injunctive norms, i.e. what most others approve or disapprove, and descriptive norms, i.e. what most others do (Cialdini, Reno, \& Kallgren, 1990). Cialdini and colleagues (1990) have argued, and empirically shown, that it is crucial to distinguish between the is (descriptive) and the ought (injunctive) meaning of social norms, because each of them refers to a different source of motivation. Injunctive norms and moral values share the concept of how something 'ought' to be and hence of moral and social approval. The difference between moral values and injunctive social norms is that the latter always refer to modes of conduct, whereas this may not be the case for moral values. For instance, the terminal values 
freedom, equality, and sanctity of life do not refer to specific behaviours, but are commonly regarded as moral values (see e.g. Skitka, et al., 2005). Moreover, moral values may be conceptualized as the general and trans-situational principles that guide injunctive norms (cf. Power, 2004). For instance, an injunctive norm that prohibits stealing is likely to be guided by the moral value of individual ownership or, in a moral community, of trust. Hence, injunctive social norms are specific prescriptions or proscriptions of how to behave in a certain way in a particular situation, whereas values, including moral values, are the general guidelines that may provide a rationale for why these behaviours are enacted in a given culture (cf. Smith, Peterson, \& Schwartz, 2002).

It may appear from the description above that a proscriptive morality (with its focus on negative outcomes) has more weight in guiding individuals' moral behaviour than a prescriptive morality. Although a proscriptive morality is more condemnatory and strict than a prescriptive morality, and often regulated by the legal system, a prescriptive morality is of equal importance for societies. Societies do not only want people to avoid bad behaviours, but also to engage in good deeds (Janoff-Bulman, et al., 2009). The mandatory nature of laws, for example, is not suitable for this kind of morality. Instead, prescriptive morality is more regulated through norms and values in regard to role expectations and obligations, and this in turn is likely to be influenced by the cultural context. A prescriptive kind of morality and its possible cultural variation is one of the main foci in this thesis. 


\section{Culture and Morality}

No topic is more central to an understanding of culture than perhaps morality. Indeed, it is often difficult to delineate where culture ends and morality begins as culture and morality share an intricate and intimate relationship.

- Joan G. Miller (2001, p. 151)

Definitions of what exactly constitutes culture vary greatly, similarly to definitions of morality. Whereas the problem of defining morality is partly due to different epistemological positions (discussed in more detail below), the problem of defining culture lies more in different focal points of what to include or exclude in the definition (for discussions see, for example, Jahoda, 1984; Rohner, 1984; and Segall, 1984). Evidence for the problem of defining culture was first provided by Kroeber and Kluckhohn (1952): After performing a thorough literature search, they were able to compile a list of 164 different definitions of culture, recently updated by Baldwin, Faulkner, Hecht and Lindsley (2006) to 313 definitions. Nevertheless, there are commonalities in these definitions which may be regarded as necessary elements if one wants to refer to 'culture': culture is passed on by exemplary behaviour rather than inherited; it incorporates values, ideas and other symbolic meaning systems; it distinguishes one group from another since it contains something that is shared by individuals of a group (cf. also Rohner, 1984).

The concept of cultural values has played a central role in differentiating one cultural group from another in cross-cultural research (cf. Gelfand, et al., 2006; Smith \& Schwartz, 1997). Contemporary cross-cultural studies of values use mostly structured questionnaires (Smith \& Schwartz, 1997). Cultural values are here commonly defined as the socially shared, abstract ideas about what is good, right, and desirable in a society or cultural group (Smith \& Schwartz, 1997). Values are a useful concept for cross- 
cultural research because they can be expressed in a decontextualized manner (Smith, et al., 2002), meaning that respondents can be asked about their values without specifying the context, which facilitates considerably cross-cultural comparisons. In contrast, behaviours and norms are always expressed within a certain context and the interpretation of behaviour may change depending on the context and also on the culture.

Besides these very general elements, there are also researchers who define or refer to culture in a way that is closely related to morality. For instance, Matsumoto (2007) says that culture is something that "prescribes limits and broad guidelines for acceptable personal and social behaviour" (p. 1302) which resembles a prescriptive kind of morality put forward earlier. In a similar vein, the anthropologist Shweder (2003) regards culture as shaping people's notion of what is good, moral and virtuous. Culture provides group members with answers to fundamental questions such as how things should be, and what is the right course of behaviour and values (Brewer \& Chen, 2007). In this way, morality can be regarded as one of the culture's rule system (Eckensberger, 2007). Henry and Reyna (2007) expressed this intrinsic relationship between culture and morality as follows:

\begin{abstract}
When a person or group behaves in ways that are consistent with important cultural ways, they demonstrate that they are upholding the moral foundations of the society and contributing to the overall benefit of its members [...] On the other hand, those who violate values threaten the stability and moral foundations of that society and are therefore likely to be shunned or ostracized from that group.
\end{abstract}

(Henry \& Reyna, 2007, p. 293)

Haste and Abrahams (2008) pointed out that it is the early experiences with care givers and peers that play an important role in shaping conceptions of the self and culturally based understandings of right and wrong. Each culture has its own values and expectations concerning its members (Zavalloni, 1980) and socialisation processes have the function that members of a society are taught to behave according to those established norms and values. 
Hence, what has been mentioned so far as necessary elements for defining culture (sharedness, symbolic meaning systems, socialization) seems to apply equally to morality. In fact, some theorists in moral psychology have pointed out that culture and morality are intrinsically related to each other in a way that morality is a constitutive feature of the culture concept (e.g. Eckensberger, 2007; Miller, 2001). Culture cannot be defined or investigated without taking into account morality, and morality cannot equally be understood without reference to the culture concept. Definitions of moral and cultural values also seem to be intertwined when cultural values are seen as the socially shared, abstract ideas about what is good, right, and desirable in a culture (Smith \& Schwartz, 1997) and moral values as the beliefs "shared in a social group about what is good or right" (Maccoby, 1968, p. 229). If what is moral is cultural and what is cultural is - at least in part - moral then it seems difficult to distinguish one term from the other.

\section{Relevance to this Thesis}

The position adopted in this thesis is that when the moral domain is investigated in different cultures, then culture is simultaneously studied. However, it needs to be pointed out that this conclusion is based upon the view of cultural and cross-cultural psychologists as referenced above. Moral psychologists adopting a universalist perspective on morality, regard cultural conventions of 'right and wrong' as operating on a lower level of morality (e.g. Kohlberg, 1984) or as not pertaining to the moral domain at all (e.g. Turiel, 1983).

A main focus in this thesis lies in a critical examination of the values concept as it has mainly been employed and studied in cross-cultural psychology. The novel contribution of this thesis is to combine moral psychology with values research, with the final aim of identifying cultural similarities and differences. The difficulty of this endeavour lies in the fact that the term 'moral values' combines two distinct research 
traditions (Eckensberger, personal communication, August 28, 2007), i.e. research in moral psychology that is mostly not concerned with the concept of values, and research in social psychology/ cross-cultural psychology on values that is mostly not concerned with assessing the concept of morality. Not only is the concept of moral values neglected in some of the theories in moral psychology (e.g. Kohlberg, 1984), but also is the variable culture disputed as having an effect on morality (e.g. Kohlberg, 1984; Turiel, 1983, 2002). The social psychological (e.g. Rokeach, 1973) and cross-cultural (e.g. Schwartz, 1994b) literature on values face yet another issue: there is a mismatch between defining values in moral terms but operationalizing them as personal preferences. These two main issues will be explained in more detail below, followed by the presentation of a value measure that has been widely employed in cross-cultural studies, i.e. the Schwartz Value Survey, (SVS, Schwartz, 1992) and that has also been used in this research to study moral values.

I begin with a brief introduction to moral psychology and I will focus in particular on the question of cultural variation in morality. The controversy of whether culture is related to morality is fundamental since it tackles not only the issue of defining constructs, but also of epistemology, i.e. beliefs about what is true moral knowledge, how it is acquired, and how it can be studied. The epistemology in moral psychology can be roughly distinguished into a normative and descriptive account. In the following section, I will briefly outline these two perspectives and then position my own research. 


\title{
Moral Psychology
}

\section{The Normative Account}

\begin{abstract}
Moral psychology is controversial stuff. Every psychologist who studies moral topics has had the experience of trying to share some fascinating nuance of his or her research with a (non-academic) friend only to encounter stiff resistance. The friend is not ready to concede the existence of morality in the first place, what morality means in the second and what gives the scholar any authority to determine right or wrong in the third. Controversial issues pervade the academic study of moral topics just the same and have since the field's inception.
\end{abstract}

- Jeremy A. Frimer and Lawrence J. Walker (2008, p. 333)

In the normative account, morality and culture are seen as mutually exclusive (see e.g. Turiel, 1983). This is based on the assumption that there is a universal morality which can be attained through rational reflection, and which may or may not be reflected in social conventions. The epistemological perspective is that qualities such as goodness and rightness are real and knowable and that moral statements can therefore be objectively determined as either true or false (see Gewirth, 1984). This kind of moral cognitivism was the predominant position in the study of morality in the 1960's (Shweder \& Haidt, 1993) and its most prominent representative is Lawrence Kohlberg. Kohlberg (1984) further developed Piaget's (1932/1965) theory on children's moral reasoning, which proposed that there are two sources of moral obligation: a heteronomous orientation where the child's reasoning is dominated by its own egocentrism within the context of the adult and child relationship, and an autonomous orientation where children develop a more equilibrated understanding of justice in the context of peers who are seen as equal. Drawing upon this distinction, Kohlberg provided a more detailed model of moral reasoning by distinguishing six successive stages of moral development. The three major phases were called preconventional, conventional and postconventional morality. 
Kohlberg (1984) was not concerned with moral values, but only with the formal aspects of moral development, i.e. the different stages that lead to moral maturity (see also Pittel \& Mendelsohn, 1966). Nevertheless, each phase may be associated with a certain value orientation. In the preconventional phase, the child judges behaviour in a self-focused manner, i.e. right or wrong is determined in terms of personal gain and loss. Moral values that may be associated with this stage are those of an hedonistic kind. In the second phase, the child judges behaviour in terms of the known convention or "what people would say" is right or wrong, which therefore may depend on the cultural context. Trust, loyalty, respect, and gratitude are the guiding moral values in this stage (cf. Krebs \& Denton, 2005). In the final - postconventional - phase, the child judges behaviour according to internalized moral principles. This final phase of moral reasoning must be based upon the principle of justice to qualify as truly moral. The principle of justice as the pinnacle of morality had been derived from philosophy- more precisely from Kant's (1785/1994) deontological theorizing about what constitutes morality. Hence, morally mature reasoning must be based on the moral values of rights, interpersonal justice and fairness which is expected to be universally valid. Kohlberg tested his model of moral development by interviewing children and adults in the USA about hypothetical moral dilemma and asking them how they would react and why they would react in this way.

In the years after Kohlberg's (1958) doctoral dissertation, some researchers examined whether the stages of moral reasoning were associated with the endorsement of certain values (e.g. Diessner, Mayton, \& Dolen, 1993; Gorsuch \& Barnes, 1973; Helkama, 1982). Helkama et al. (2003) recently reviewed the literature on the empirical link between personal values and Kohlberg's stages, concluding that the pattern of relationships between moral reasoning and value priorities does not allow distinguishing a set of values that operates distinctively at 'stage two', 'stage three' and 'stage four'. It 
rather appears that Kohlberg's stages and personal value priorities assess something different.

Despite the enormous impact Kohlberg's model has had on the research community and on subsequent studies in moral psychology (see, for example, Turiel, 2008), his theory has also been criticized as being sexist (Gilligan, 1982), confusing the moral domain with the social conventional domain (e.g. Turiel, 1983), as being culturally biased (e.g. Shweder, Mahapatra, \& Miller, 1987) and neglecting the content of morality (cf., for example, Walker \& Pitts, 1998b).

The US-American feminist Gilligan (1982) argued against Kohlberg's theory and findings which showed that women were typically ranked on lower stages and hence were more deficient in the morality of justice than men. She proposed that there may be two principles which underlie moral reasoning: the principle of justice and the principle of care. She showed that US-American females see justice and fairness as less important and are more concerned with prosocial responsibilities and the moral values of trust, loyalty and respect which Gilligan subsumed under the principle of care. However, subsequent research did not reveal consistent gender differences in moral reasoning (cf. Walker, 1991). Additionally, Gilligan's theory does not consider possible cultural variations in the ethics of care and justice. Morality is still placed into an universalistic framework in which all cultures embody the same patterns of structural inequalities associated with gender which are supposed to have an effect on moral outlooks (Miller, 2001).

Turiel et al. (e.g. Nucci \& Turiel, 1978; Turiel, 1983, 2002) have criticized Kohlberg for not clearly separating social conventional rules from moral rules. They developed the Social Domain Theory which posits that there are three different domains which differ in the extent to which behaviour is socially regulated: the personal, the social conventional and the moral domain (Nucci \& Turiel, 1978; Turiel, 1983). The 
personal domain is defined by actions considered to be outside the realm of societal regulation and moral concern (e.g. watching TV on a sunny day). Social conventions are defined as arbitrary acts that are based on consensus, and which serve the function to coordinate social interactions within social systems (e.g. whether or not to address the teacher by his/her first name). They may produce culturally idiosyncratic rules and are arbitrary because they do not have an intrinsically prescriptive basis, i.e. alternative actions can serve the same function. The moral domain is defined as "prescriptive judgments of justice, rights and welfare pertaining to how people ought to relate to each other" (Turiel, 1983, p. 3; e.g. not to hit other people with no reason). Turiel's (1983) early studies were based on interviews with children and adolescents in the USA about transgressions of moral, social conventional and personal rules. On the basis of his definitions of what constitutes morality, he specified a priori which acts were to be counted to which domain of social regulation. He measured the domain distinction with a number of questions such as rule contingency (is it $\mathrm{OK}$ if there was no rule about it?), rule relativity (would the act be $\mathrm{OK}$ in another country?), deserved punishment (should the transgressor be punished?) and evaluation of seriousness (how bad is the transgression?).

In a series of studies, Turiel and his colleagues were able to show that children experience only moral rules as obligatory, universal and unalterable. Social conventional rules however were regarded as based on social consensus and seen as alterable (see Turiel, 1983, for a review of studies). This domain distinction was even found with children as young as two years old (Smetana, 1981) and with crossculturally diverse samples (e.g. Nucci, Turiel, \& Encarnacion-Gawrych, 1983) which indicate an innate and universal kind of morality with moral values centred around the principle of justice. Hence, contrary to Kohlberg's (1984) proposition, Turiel et al. 
showed that moral development is not a process of progressively differentiating morality from social convention (see e.g. Nucci \& Turiel, 1978).

Despite compelling arguments and empirical evidence, Turiel's strict differentiation of the moral, social conventional and even personal domain has been challenged. Smetana (1982), for instance, found that a women's reasoning for or against abortion depends in many circumstances on whether the woman views abortion as a personal or moral issue. Research conducted by cultural and cross-cultural psychologists has also challenged Kohlberg's and Turiel's assumption that the moral domain is universally centred on the moral value of justice. Cross-cultural studies found that in different cultures and at different times people are morally mature when they respect principles other than justice, such as their codes of honour, their karma, or their desire to actualize their potential as human beings (see Campbell, Christopher, \& Bickhard, 2002). Cross-cultural research testing the cultural invariance of Turiel's Social Domain Theory has not always found the clear-cut division between morality and social convention (e.g. Nisan, 1987, in Israel; and Shweder, et al., 1987, in India). These studies have shown, that the intrinsic criteria for 'harm' and 'suffering' may not be as objective as proposed by the Social Domain Theory and that the distinction of moral and social conventional rules are in some cultures blurred (see also Krebs, 2008). For example, it is highly immoral for a son to cut his hair during the ten days that follow the death of his father among Brahmans in India (Shweder, et al, 1987). Hence, what causes harm is often understood from a localized normative point of view (see also Miller, Bersoff, \& Harwood, 1990).

The problem with Piaget's, Kohlberg's and Turiel's research paradigm is that they defined morality through the adoption of externalistic criteria (see also Pittel \& Mendelsohn, 1966). This means that the standards for judging a person's morality were taken from sources outside the person being assessed (a Kantian philosophy) and neither 
her own moral values nor her cultural context were taken into account as possible sources of alternative moralities.

Instead of normatively specifying as a researcher what morality is (or should be) for all human beings, some psychologists advocate to simply describe the different moral conceptions that exist (Haidt, 2001; Walker \& Pitts, 1998b). This does not necessarily imply the adoption of a moral relativism position. The goal of moral psychology is simply viewed as understanding the values that human beings acquire, even if their moral ideology is immoral and detrimental to others. As Campbell, Christopher and Bickhard (2002) stated: "Acknowledging a plurality of moral conceptions does not mean endorsing all as equally right or good, [but ...] to explain how human beings could arrive at such diverse endpoints through the normal process of development" (p. 798). In order to do so, it is necessary to change the concept of morality from an individual perspective, where the individual is isolated from the society and its influence, to a socio-cultural perspective of morality (Tappan, 2006). This allows (1) broadening the moral domain from the issue of harm (and justice) to social expectations and social conventional values, and (2) relying on what laypeople commonly regard as moral without subjugating their responses to philosophical definitions (see also Walker \& Pitts, 1998b).

\section{The Descriptive Account}

Shweder and colleagues (e.g. Shweder, et al., 1987; Shweder, et al., 1997) developed the Social Communication Theory which takes the cultural context into account in the development of morality. Children acquire morality through engagement in and perception of social communications and interactions which contain cultural judgments and ideologies. Social practices contain the main sources of moral content and can be perceived as moral imperatives, as research in India has shown (Shweder, et 
al., 1997). Shweder et al. (1987) found in qualitative interviews that the concept of social conventions, in relation to arbitrary rules that are based on social consensus and that are alterable, was not meaningful to Indians of any age. With increasing age, Indian children saw more and more issues as a question of universal moral principles, whereas US-American children showed the opposite tendency, i.e. that the same issues pertained to social conventions. Based on their findings, Shweder et al. (1997) proposed that there are two alternative types of morality to the justice-based morality that stem from discourses with different conceptions of the self and social relations. Together with the justice-morality, they were called the 'big three' of morality: the ethics of Autonomy, Community, and Divinity.

The ethic of Autonomy is similar to Turiel's (1983) justice-based morality with moral values related to justice, human rights and prevention from harm. The moral agent is defined as an autonomous person who is free to make his/her own choices, only restricted by concerns whether a behaviour would inflict harm on others or restrict their rights. The ethic of Community defines the moral agent in terms of their membership in social groups (e.g. family) and the obligations that go along with this membership. Moral values are those that benefit the functioning of the community, such as duty, honour, chastity, respect, modesty, and self-control (see also Vélez García \& OstroskySolís, 2006). The ethic of Divinity defines the moral agent as a spiritual entity who aims to follow divine laws trying to achieve moral purity. Moral values are grounded in divine law and often derived from religious authorities and texts (Jensen, 1995), and are those that protect and dignify the divinity inherent in people (Vélez García \& OstroskySolís, 2006). These ethics vary in their emphasis and distribution across and within cultures. The ethic of Autonomy has been found to be dominant in the Western moral discourse, whereas the ethic of Community is more prominent in collectivistic-oriented cultures such as Asian cultures where there is a moral obligation in regard to the 
sensitivity of others' needs, and individual self-fulfilment is regarded as secondary (e.g. Chiu \& Hong, 1997). Chiu et al. (1997) labelled these two different ethics as a 'rightsbased morality' (ethic of Autonomy) and a 'duty-based morality' (ethic of Community).

Although the moral theories discussed so far - i.e. Gilligan's Ethic of Care, Turiel's Social Domain Theory and Shweder et al's Big Three of Moraliy - differ in critical ways from Kohlberg's Cognitive-developmental Theory, their emphasis remains on moral reasoning (Walker \& Pitts, 1998b), i.e. people's explanations for why a behaviour is right or wrong. Researchers have recently pointed out that moral reasoning is not sufficient for a comprehensive understanding of moral functioning (e.g. Frimer \& Walker, 2008). In this context, it has been proposed that morality consists of two different processing systems of which only one pertains to moral reasoning (Haidt, 2001; Lapsley \& Hill, 2008): (1) the explicit/ deliberative process, and (2) the implicit/ tacit process. The explicit process has been widely studied in the past by analyzing individuals' reasoning on moral dilemmas or moral transgressions (e.g. Kohlberg, 1984; Turiel, 1983). The implicit process operates at another more unconscious level; it is automatic and relatively effortless. This process has only recently drawn attention as a research focus (Lapsley \& Narvaez, 2004) partly triggered by Haidt's (2001) Social Intuitionism Theory.

Haidt (2001) argues that people rely on gut feelings to distinguish right from wrong. Moral intuitions are both innate and learned or enculturated respectively. Moral intuitions can be defined as "the sudden appearance in consciousness of a moral judgment, including an affective valence (good-bad, like-dislike), without any conscious awareness of having gone through steps of searching, weighing evidence, or inferring a conclusion" (p. 818). Haidt regards morality, like language, as a major evolutionary adaptation that is universal, but requires input and shaping from a particular culture. His Social Intuitionism Theory is social, because it emphasizes the importance of social and 
cultural influences on morality. It is an intuitionist model, because moral judgment is viewed in general as a result of quick and automatic evaluations. One of the main differences to rationalist models (morality as an explicit/ deliberative process) lies in the hypothesised causes. Haidt proposes that moral intuitions come first and directly cause moral judgment. The moral reasoning serves to rationally justify the intuition in the first place. The opposite causal relationship is assumed in the rationalist model.

Social Intuitionism Theory is consistent with recent findings from diverse disciplines such as social psychology, evolutionary psychology, anthropology, and primatology (Haidt, 2001). Empirical evidence has so far underpinned the importance of affective reactions in moral judgments. Haidt, Koller and Dias (1993), for example, found in a cross-cultural study (Brazil and USA) that 'harm' cannot explain all moral judgments that individuals make. They constructed stories in which no plausible harm was involved, for instance, cleaning one's toilet with the national flag, eating a dog or masturbating with a dead chicken. Respondents' affective reactions to these stories were better predictors of their moral judgments than their claims about harmful consequences. Haidt and Hersh (2001) found similar results when interviewing liberals and conservatives in the USA on the issue of homosexuality.

The social intuitionism model not only accommodates affectively heavily charged actions, but the entire range of moral judgments. Haidt (2001) points out that in every society people make evaluations. Many of these evaluations, with respect to virtues or goods, are applied to everyone in a certain society or to everyone in a certain social category. These virtues are obligatory in the sense that everyone (within the relevant category) is expected to strive for them. People who fail to do so are subject to social criticism or punishment. Moral judgments can then be defined as "evaluations (good vs. bad) of the actions or character of a person that are made with respect to a set of virtues held obligatory by a culture or subculture" (p. 817). Haidt emphasizes that his 
definition of moral judgment is an intentionally broad definition to accommodate different possible moral outlooks. For instance, eating a low fat diet may not readily qualify as a moral issue from a philosophical point of view, but within many healthconscious subcultures people who eat junk food are seen as morally inferior to those who eat healthy food (Stein \& Nemeroff, 1995).

Haidt's epistemological approach in researching morality is to study individual and cultural facts about moral functioning and not to impose a priori definitions of what qualifies as truly moral. This approach is more bottom-up, both empirically and conceptually (Haidt \& Joseph, 2007). He takes it as given that what people think are their moral concepts are in fact moral concepts - rather than separating them into moral and social conventional concepts on the basis of externalistic criteria. By doing so, he leaves room for a culturally relative morality. Nevertheless, his position is also that some aspects of morality are universal. In line with evolutionary theorists (e.g. Richerson \& Boyd, 2005), he suggests that a universal morality evolved because it was most effective in upholding mutually beneficial cooperative relations. A culturally relative morality evolved because people from different cultures experience different adaptive problems in different ways, and because norms may emerge from social and cultural processes that stand only in indirect relationship to biological evolution.

Haidt's social intuitionism model of morality is highly flexible, since there are no a priori assumptions of what counts as morally relevant. Haidt empirically evokes moral evaluations through vignettes containing short stories about behaviours that may be intuitively judged as immoral (see e.g. Haidt, et al., 1993). However, Social Intuitionism Theory can also be applied to the concept of values. Batson, Engel and Fridell (1999), for example, used the values freedom and equality and gave participants false physiological feedback about their emotional reactions when listening to stories in which these values were threatened. Respondents relied on their assumed visceral 
reaction to make value-relevant decisions in subsequent tasks. Another very different approach would be to ask individuals to judge which values are intuitively morally relevant or not. This approach, with the goal to compare the outcome cross-culturally, has not been adopted so far.

More recently, Haidt et al. (Haidt \& Graham, 2007; Haidt \& Joseph, 2004) developed the Moral Foundations Theory which posits that people use five sets of moral intuitions to judge wether an issue is right or wrong: harm/ care, fairness/ reciprocity, ingroup/ loyalty, authority/ respect, purity/ sanctity. These five moral foundations provide a taxonomy for the bases of moral judgments, intuitions, and concerns. Graham, Haidt and Nosek (2009) suggest to use Moral Foundation Theory if one's goal is to study the sources of moral intuitions across cultures. Yet, if one's goal is to describe moral discourse across cultures, then Shweder et al.'s (1997) three ethics can be used and if one is interested in examining values, then Schwartz' (1992) value types are useful. In fact, the measure that Haidt and colleagues (Moral Foundations Questionnaire, Graham, et al., 2009) developed to test the different moral foundations does not assess values as instrumental or terminal goals, but the relevance of various concerns tapping into the five sets of moral intuitions (e.g. whether or not someone was harmed). Hence, it can be said that there have not been attempts so far to map the moral domain from a motivational perspective using the values concept. 


\section{Theoretical Stance in this Thesis}

As an empiricist, I believe that we can study things like life without defining them...This strategy, I believe, works just as well for the aspect of life that we call 'morality.' For empiricists, rigorously defining morality is a distant goal, not a prerequisite. If anything, I believe that defining morality at this point is more of a hindrance than a help, as it may artificially narrow the scope of inquiry...Rather than seeking out morality by the light of a philosopher's definition (Kantian or otherwise), I and like-minded scientists choose to study decisions that ordinary people regard as involving moral judgment.

- Joshua Greene (2007, October 9)

Greene's quote summarizes the position that is taken in this thesis in regard to a psychological and cross-cultural investigation of morality. This research does not take an a priori stance on what qualifies as moral and what does not from a researcher's or philosopher's perspective - this judgment is left to the respondents. This thesis is a cross-cultural investigation of what is morally relevant in different cultures. As such, the outcome of this research is to be understood as descriptive - in terms of what people in different cultures regard as morally relevant - and not as prescriptive - in terms of how morality should be construed. Equating what 'is' with what 'ought to be' would in fact mean committing the naturalistic fallacy (Moore, 1903). Hence, the study of morality is here seen as the study of agreements among people and is therefore a kind of social knowledge (cf. Haan, 1982).

Haidt's (2001) Theory of Social Intuitionism is the most flexible model of all to study this kind of social knowledge. A cross-cultural study of moral values and virtues, i.e. those that elicit judgments of good and bad, or right and wrong, should provide valuable insights into cultural differences and similarities. Despite the fact that values have played a major role in assessing cultural differences in cross-cultural psychology (see e.g. Gelfand, et al., 2006), moral values have not been a major focus in previous investigations. 


\section{Cross-cultural Psychology}

Culture is often seen as a system of values (e.g. Rohner, 1984). As a consequence, research on cultural differences and similarities is frequently centred on the concept of values (see e.g. Gelfand, et al., 2006). Cross-cultural research that takes a quantitative approach to the study of culture conceptualizes values as broad frameworks that underlie the functioning of cultural groups and aid to understand the differences between them (Smith, et al., 2002).

Similarly to the culture concept, there are many different definitions of values which amounted to 180 different conceptualizations in the 1980's (see Harding \& Phillips, 1986). Values are often so broadly defined, that anything wanted by a person is regarded as a value (Kilby, 1993). Despite all these different definitions, there are commonalities which aid pinning down the very essence of the value concept: values are always positive (Roe \& Ester, 1999) and they can be endorsed by a single person or by a collective unit (e.g. Rokeach, 1973). Values are positive, because they have an intrinsic worth. A certain value that is endorsed by one person (or collective unit) may not be regarded as something positive to another person (or collective unit), but in this case this other person (or collective unit) would not regard it as a value. Hence, the intrinsic worth of values is relative.

Many value definitions refer to values as something 'desirable' and not as something 'desired'. It will be shown that while the latter is unrelated to morality, the former has been implicitly linked to the concept of moral values. The concepts of the 'desired' and the 'desirable' will be contrasted in more detail and it will be shown that despite the consensus that values pertain to the 'desirable', the operationalization in practice often does not match the conceptualization in theory. 


\section{Values as the 'Desirable'}

\section{Man is an evaluating animal.}

- Clyde Kluckhohn (1952, p. 403)

It has often been stressed in the literature that the concept of values includes a valuation and differs from something that is simply 'desired' which can be free from an evaluation (e.g. Dewey, 1939, in his manuscript the Theory of Valuation). Something that is 'desired' can just be something that is 'wanted' by someone but it does not necessarily contain a valuation of being something positive, worthwhile, right or prescriptive (cf. Kilby, 1993). Kluckhohn (1952), for instance, in his seminal paper on values emphasized that it is critical to distinguish values as the 'desired' and the 'desirable' ${ }^{1}$. In all cultures people have desires or wants for themselves. However, this is not the same as the 'desirable' which is not just a preference, but a standard that is felt to be morally justified. Social life would be impossible without values as the 'desirable' (Kluckhohn, 1952).

'Ought' and 'should' statements do not vary randomly, but show some degree of pattern and add predictability to social life. Values are for the most part cultural products and can be distinctive of a plurality of individuals. Group values consist of socially sanctioned ends and socially approved modes and means. Values as the 'desirable' refer to goals of action which are evaluated on an approval-disapproval continuum and therefore have an affective component. They represent what individuals believe they 'ought' or 'should' desire for their own sake, or the sake of the group.

\footnotetext{
1 Hofstede (1980) later referred to the pitfall of equating the 'desired' and the 'desirable' as the 'positivistic fallacy' after Levitin (1973).
} 
Value statements can then be regarded as normative or prescriptive statements and the “cue words are right or wrong" (Kluckhohn, 1952, p. 398).

Subsequent value researchers and theorists have followed Kluckhohn's suggestion to regard values as bipolar in their valence (e.g. Rokeach, 1973) with evaluations in terms of what is good and desirable as opposed to bad and undesirable (e.g. Harding \& Phillips, 1986; Kilmann, 1981) and to relate them implicitly or explicitly to the moral realm. For instance, Smith (1969) posits that "values pertain to the desirable, the preferable, rather than the merely desired or preferred; to the realm of 'ought' rather than that of [...] 'want' (p. 102). The contemporary philosopher Louis Pojman (1995) argues that "...values are central to the domain of morality" $(1995, \mathrm{p}$. 93) and that from "our values, we derive principles" (p. 94). Kristiansen and Zanna (1994) suggested that from a psychological perspective values are people's beliefs about obligations and what they ought to do and strive for. Prominent cross-cultural and value researchers also include values as the "desirable" in their value definition (e.g. Feather, 1996, p. 222; Kluckhohn, 1952, p. 395; Schwartz, 1994, p. 21). Cultural values are commonly regarded as the socially shared, abstract ideas about what is good, right and desirable in a society or cultural group (Smith \& Schwartz, 1997).

To conclude, there is a consensus in the research community that values contain a moral connotation. Despite the widespread definition of values as the 'desirable', surveys that indeed operationalize moral values are scattered and few (Zavalloni, 1980). And therefore there are only a few empirical investigations of moral values (cf. Harding \& Phillips, 1986). The scales that exist can be put into two categories: (1) scales for which it is claimed that they measure moral values, but which do not directly assess values, but other psychological constructs such as attitudes and beliefs, and (2) measures for which values are defined in moral terms (as the 'desirable'), but are 
operationalized as personal preferences (see also Harding \& Phillips, 1986). The most relevant research falling under these two categories is summarized next.

\section{Moral Values Scales}

Research on moral values is scarce, especially in terms of more recent research. Empirical studies on moral values until the mid 1960's have been reviewed by Pittel and Mendelsohn (1966). Their review is summarized here since it provides an insight into the origins of some of the measures that are still used, and the issues associated with research on moral values. Pittel and Mendelsohn (1966) divided the developments in the measurement of moral values into three different periods. The first period from 1900 to 1930 was marked by paper and pencil tests of moral knowledge mostly used to distinguish between normal and delinquent adolescents (e.g. Brotemarkle, 1922). From 1932 to the 1950 's, moral values were examined in the scope of broader theoretical orientations. There was a methodological shift from paper and pencil tests of moral knowledge to studying the formal aspects of the child's moral behaviour through interviews and observation (e.g. Piaget, 1932). The second phase was also marked by omnibus investigations in the USA in which personality was assessed primarily based on psychoanalytic and behaviouristic models. The main interest was to investigate individual differences in 'normal' subjects on diverse aspects related to morality, such as impulse control, guilt and judgments of moral transgressions. This was also the era in which scales were developed to assess moral values which are still in use - in the same or a more contemporary version. One such example is Crissman's (1942) scale, that includes a number of 'immoral' behaviours (e.g. 'having sex while unmarried' or 'taking one's own life'). The third period, the 1960's and 1970's, were characterized by the use of projective techniques such as the Rorschach test to examine psychoanalytical moral concepts like the super-ego. 
Pittel and Mendelsohn (1966) heavily criticized all these attempts to measure moral values. Among their main arguments were that the investigators decided a priori what is moral and what is not; the majority of instruments have not been standardized or validated; and often knowledge of legal, moral and ethical standards were assessed rather than the individual's moral beliefs. Their conclusion was that there had been insufficient effort to conceptualize the nature of moral values.

Since the 1970's, there have been a number of cross-cultural studies mainly using a version of Crissman's (1942) scale in which respondents were asked to evaluate a number of different behaviours in terms of their 'rightness' or 'wrongness'. Tomeh (1968), for instance, used the Crissman scale in a cross-cultural sample with respondents from the Middle East (Christians and Muslims) as well as an American sample. She found that Middle Easterners were more severe in their overall judgment of immoral behaviours, while Americans were more severe in codes regarding individual responsibility. Klinger (1962) also studied offences with a scale more adapted to students' (immoral) behaviour (developed by Mueller, 1958) in a cross-cultural sample of male students living in the USA (American Protestants, Arab Moslem, Chinese nonChristians, Indian Hindus, Roman Catholics from Latin-America and Turkish Moslems). He found certain behaviours to be condemned by all (e.g. drunk driving) and others to be judged differently depending on the culture (e.g. premarital sex).

More recent research has been conducted with data from the European and World Value Survey in which a more contemporary version of Crissman's scale- the Morally Debatable Behaviour Scale (MDBS, Harding \& Phillips, 1986)- has been used to assess people's moral outlook. Using these data, individuals' moral attitudes have, for example, been linked to religiosity (Nevitte \& Cochrane, 2006) and also studied in terms of their change over time (Moors \& Wennekers, 2003). 
When reviewing mono-cultural studies that claim to have measured moral values, it becomes evident that the term moral values is stretched to include a number of other concepts such as norms and schemas (Biel, Fransson, \& Dahlstrand, 1997), ethical ideologies (Singh \& Forsyth, 1989), moral attitudes towards transgressions (Bovasso, Jacobs, \& Rettig, 1991; Halpern, 2001; Hardy, Padilla-Walker, \& Carlo, 2008) and more specifically towards aggression, sexuality or discipline (Rosenthal, 1955; Schmidt, 1988). Further assessments of individuals' moral values include open-ended questions (Hogan \& Dickstein, 1972) and scenarios focusing on the moral value of justice (Herndon Jr., Fraedrich, \& Yeh, 2001). Other researchers used personal value measures such as the Rokeach Value Survey (Rokeach, 1973) or Chinese Value Survey (Chinese Culture Connection, 1987) and claimed that some of the values in these inventories assess moral values, however without further conceptual or empirical justification (e.g. Shen \& Yuan, 1999; Sikula \& Costa, 1994).

To conclude, Pittel and Mendelsohn's (1966) main criticism of moral value measures in the 1970's can also be applied to the more recent publications: it is the researcher who defines what is moral or not (e.g. Hardy, et al., 2008; Herndon Jr., et al., 2001; Schmidt, 1988; Shen \& Yuan, 1999; Wojciszke, 1997); what is moral is often equated with a strict moral outlook on sexual and criminal behaviours (e.g. Halpern, 2001; Singh \& Forsyth, 1989). In addition to that, values are often not operationalized as end-goals or modes of conduct, but inferred from other constructs such as moral attitudes (e.g., Bovasso, et al., 1991; Moors \& Wennekers, 2003; Nevitte \& Cochrane, 2006). This confusion of concepts impedes a deeper understanding of their relationship, such as whether people's specific attitudes can be predicted from their more general value orientation (see, e.g., Rohan, 2000). 


\section{Values as Terminal and Instrumental Goals}

One of the most innovative contributions to values research is attributable to Rokeach's work (Rokeach, 1968, 1973). He defined a value as "an enduring belief that a specific mode of conduct or end-state of existence is personally or socially preferable to an opposite or converse mode of conduct or end-state of existence" (p. 5). One of his major contributions lies in the fact that he does not infer values from responses to specific attitude statements, but measures them directly. The survey he developed, i.e. the Rokeach Value Survey (RVS, Rokeach, 1973), operationalizes values by explicitly naming them as either terminal goals (represented by nouns, e.g. freedom) or instrumental goals (represented by adjectives, e.g. helpful). He gathered his data by presenting respondents two lists of terminal and instrumental values (with 18 values in each), and asking them to rank order the values "in order of their importance to YOU, as guiding principles in YOUR life" (Rokeach, 1973, p. 27).

Rokeach proposed a theoretical taxonomy of values by subdividing terminal values into those with a personal or social focus, and instrumental values into those referring to one's competence or morality. However, a factor analysis on the basis of data from 1409 American adults could not support this theoretical distinction of values. He concluded that "it is unlikely that the 36 values can be effectively reduced to some smaller number of factors" (Rokeach, 1973, p. 44). This is one of the main criticism that has been raised in regard to the RVS. Single-item measures are problematic according to psychometric theory. No single item is a pure measure of the construct of interest, because each reflects error, some attributable to other irrelevant constructs or to random fluctuation (Braithwaite \& Scott, 1991). A further point that can be added to this critique is, that the survey assesses what is personally important and not what is regarded as prescriptive. 
Hence, despite the widespread consensus in the literature that values refer to what is 'desirable' and therefore to the moral realm and not the 'desired', which is a personal preference, many value measures (including the RVS) do not operationalize values as a conception of the 'desirable'. Kilmann (1981), for instance, criticized that it is often the personal importance of values that is assessed and that the outcome can be interpreted as what people merely want in their lives. Kilmann (1981) concludes in his review on value measures that "researchers may define their concept of values a particular way, the critical issue is how the value is operationalized in the measurement process" (p. 956) which usually does not match the concept of values as the 'desirable'. Smith (1969) has also criticised popular value inventories for focusing on personal preferences and neglecting the social aspect of values. Cross-cultural research on values has examined almost exclusively value importance (Smith \& Schwartz, 1997).

There have been a few studies that have addressed this very issue. Mueller and Wornhoff (1990), for example, measured personal values (the 'desired') and social values (the 'desirable') in regard to different aspects such as work ethic and altruism. Personal values (e.g. independence) were assessed in terms of what people like, prefer or regard as personally important and social values in terms of whether they think other people should value these goals. Although, this study is very promising in regard to researching prescriptive values, the authors used only seven value items, and their list is therefore not exhaustive. Another relevant study has been conducted by Wojciszke (1997). He asked individuals whether some of the instrumental values of the RVS were related to competence or morality, the latter defined as traits pertaining to breaking or maintaining moral rules and/ or doing good or bad things for another person. He found that collectivistic values as defined by Schwartz and Bilsky (1987) and Schwartz (1992) were more strongly related to morality, whereas individualistic values were more related to competence. This study is one of the few that comes close to the aims of my thesis. 
Yet, the exclusive focus on instrumental values, the use of a single-item measure and the lack of a more precise theory about the underlying value system structure, makes it impossible to understand how the rating of one value item is related to the rating of another value item (cf. Schwartz, 1996). A theoretical pattern of value responses is also crucial for predictions of how ratings of a particular value type are related to other variables. Furthermore, a theoretical value structure is necessary to test for crosscultural equivalent meaning of values prior to investigating cross-cultural comparisons.

More recently, a value theory and corresponding measure has been developed that meets these stringent requirements (Schwartz \& Bilsky, 1987; 1990). The Schwartz Value Survey assesses individuals' personal values by asking them to rate terminal and instrumental values in terms of their importance to them as guiding principles in their lives. Schwartz and his colleagues (e.g. Schwartz \& Bardi, 2001) define values as “desirable, transsituational goals, varying in importance, that serve as guiding principles in people's lives" (pp. 269, see also Schwartz, 1992, for a fuller elaboration). Hence, Schwartz intentionally links values as the 'desirable' with their operationalization through personal importance ratings. His value definition can be viewed in terms of both the 'desired' and the 'desirable' (Rohan, 2000). He derives cultural values through the statistical aggregation of personal values which are claimed to reflect shared conceptions of what is good and desirable in a culture (Schwartz, 2004).

Recently, researchers have used Schwartz' list of values, but modified the response scale to assess, for instance, societal values rated as how important the values are to a particular society (Bernard, Gebauer, \& Maio, 2006), social value systems assessed as how important each value is for most people in the respondents' country of birth (Fischer, 2006), and perceived cultural values rated as how important each value is for an average person from a particular cultural group (Wan, Chiu, Peng, \& Tam, 2007; Wan, Chiu, Tam, et al., 2007). So far, there has been no study in which the response 
scale has been changed to assess moral values. Yet, there is clearly a precedent for such appropriate modification.

Schwartz' theory, his survey for the assessment of values, and his concept of cultural values play a central role in contemporary value research. They were used in this thesis and will therefore be presented in more detail below.

\section{Schwartz' Value Survey and Theory}

Schwartz and Bilsky $(1987,1990)$ developed a theory about how instrumental and terminal values are organized in a coherent value system. One of their main statements is that the value system is organized in a quasi-circumplex structure. As such, value priorities have an effect on all the other values in the value system. Neighbouring values in the circle are closely related in their underlying meaning and will most likely be endorsed in a similar fashion. However, opposite values in the circumplex exhibit opposite underlying meanings and are therefore most likely to be endorsed in opposite ways. The theory was first developed for the individual-level (see also Schwartz, 1992). However, since values are presumed to encapsulate the aspirations of both individuals and societies (Braithwaite \& Scott, 1991; Parsons \& Shils, 1952), Schwartz (1994) also developed a theory for the culture-level. Despite the fact that there are two different theories, the hypothesized underlying dynamic of value conflicts and compatibilities is the same at both the individual- and culture-level. The next sections outline the two theories and also the measurement of values in more detail.

\section{The Individual-level}

The Theory. Schwartz and Bilsky $(1987 ; 1990)$ proposed a nearly universal theory of human values. They suggested that there are three universal human needs to which all individuals must respond: (1) biologically based needs of the organism, (2) 
social interactional requirements for interpersonal coordination, and (3) social institutional demands for group welfare and survival. Values are the cognitive representations of these needs and they can be categorized into different motivational goals. Individuals differ only in the relative importance they place on these universal motivational goals.

Schwartz (1992) later proposed that two higher order motivational dimensions structure the value system. Each of the two dimensions represents a fundamental human problem that needs to be solved. And each dimension is marked by a bipolar value orientation which stands for the fundamental value conflict. One dimension is labelled as openness-to-change versus conversation and relates to the conflict of being motivated to follow one's own interests (intellectual and emotional) or to follow other's interests by "preserving the status quo" (Schwartz, 1992, p. 43). The second dimension is labelled as self-enhancement versus self-transcendence and represents the conflict of being motivated to enhance one's own personal interests (even at the expense of others) or to transcend selfish concerns and promote the welfare of others (both close and distant ones as well as nature). Schwartz (1992) proposed ten value types which represent universal motivational goals and which are arranged along these two dimensions: power, achievement, hedonism, stimulation, self-direction, universalism, benevolence, conformity, tradition, and security. Figure 1.1 shows the location of these value types along the two higher order value dimensions. 


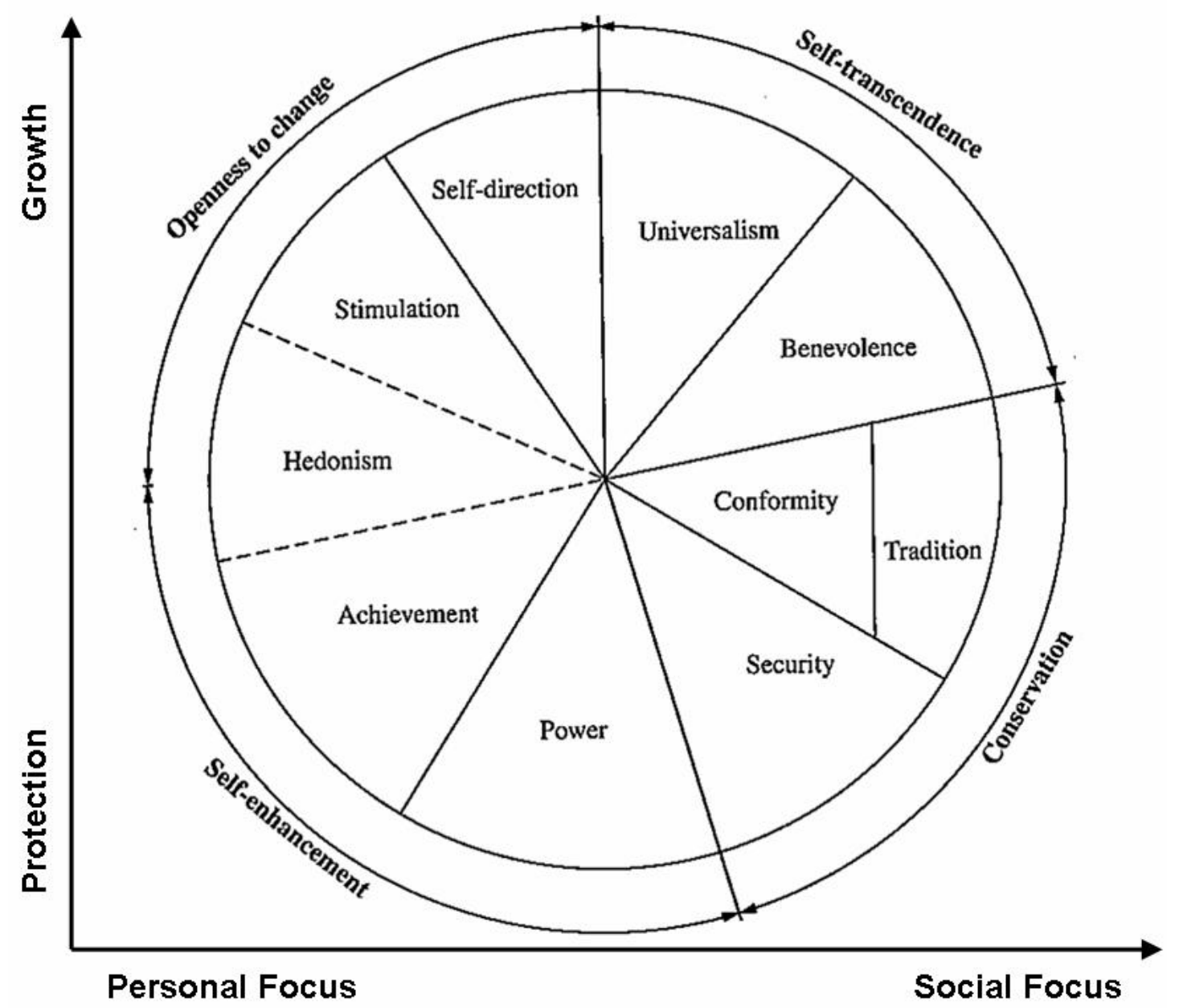

Figure 1.1: Circular model of relations among ten motivational value types at the individual-level (adapted from Schwartz, 2006b, p. 965; Schwartz \& Sagiv, 1995, p. 96).

Note. The dashed lines delineate the region occupied by the value type 'hedonism', because Schwartz (1992) indicated that this value type can be considered as part of both adjacent value types.

The Survey. In order to empirically test the value theory, Schwartz (1992) developed the Schwartz Value Survey (SVS) which has become a 58 item value measure (Schwartz, 2007a) assessing the ten motivational goals. Table 1.1 shows the motivational goals, their definition and the value items through which they are operationalized in the survey. Respondents are instructed to rate how important each of the values is to them as guiding principles in their lives. Schwartz (1992) conducted an 
extensive cross-cultural study which so far has amounted to samples from 66 nations composed of secondary school teachers, university students and the general public (Schwartz, 2007b). Using smallest space analysis, which is a non-metric multidimensional scaling technique (van de Vijver \& Leung, 1997), he demonstrated that the hypothesized value structure was present in most of the cultural samples (Schwartz, 1992; Schwartz \& Sagiv, 1995). Of all value items, 45 indicated a nearly universal meaning by clustering consistently together in the hypothesized way in at least $75 \%$ of the samples (Schwartz, 1992). Schwartz recommended using only these 45 values since cross-cultural equivalence had been established only for them (see Table 1.1.).

The relation of value types to other variables can empirically be investigated by constructing indexes of each value type. This is done by averaging the ratings of the specific value items that are representative of the respective value type (cf. Schwartz, 1992). The value type indexes can then be examined in relation to other variables through correlation or regression analysis, for example. The circumplex structure of the theory implies that there is a sinusoidal curve to expect when correlating the value types with other relevant variables. This curve follows the order of the value types around the circle with first decreasing and then increasing correlations when moving away from the main diagonal (Schwartz, 1992, 1996). The highest positive correlations are to be expected with the value type that is theoretically related to the variable of interest and the highest negative correlation with the value type that is opposite to it. For example, Schwartz (1996) investigated the relationship between individuals' personal values and their readiness to cooperate with another person in an adaptation of the prisoner's dilemma game. He found that benevolence was most positively correlated with cooperation and power most negatively with it. Moreover, the order of the correlations followed the order around the value circle from benevolence to power. 
Table 1.1: Value types, their definitions and cross-culturally equivalent value items that represent them.

Value domain

Motivational goal

Value items

\begin{tabular}{|c|c|c|}
\hline $\begin{array}{l}\text { UNIVERSALISM } \\
\text { (UN): }\end{array}$ & $\begin{array}{l}\text { Understanding, appreciation, tolerance } \\
\text { and protection for the welfare of all } \\
\text { people and for nature. }\end{array}$ & $\begin{array}{l}\text { broadminded, wisdom, social justice, } \\
\text { equality, a world at peace, a world of } \\
\text { beauty, unity with nature, protecting the } \\
\text { environment }\end{array}$ \\
\hline $\begin{array}{l}\text { BENEVOLENCE } \\
(\mathrm{BE}):\end{array}$ & $\begin{array}{l}\text { Preservation and enhancement of the } \\
\text { welfare of people with whom one is in } \\
\text { frequent personal contact. }\end{array}$ & $\begin{array}{l}\text { helpful, honest, forgiving, loyal, } \\
\text { responsible }\end{array}$ \\
\hline $\begin{array}{l}\text { CONFORMITY } \\
(\mathrm{CO}):\end{array}$ & $\begin{array}{l}\text { Restraint of actions, inclinations, and } \\
\text { impulses likely to upset or harm others } \\
\text { and violate social expectations or norms. }\end{array}$ & $\begin{array}{l}\text { politeness, obedient, self-discipline, } \\
\text { honoring parents and elders }\end{array}$ \\
\hline $\begin{array}{l}\text { TRADITION } \\
\text { (TR): }\end{array}$ & $\begin{array}{l}\text { Respect, commitment and acceptance of } \\
\text { the customs and ideas that traditional } \\
\text { culture or religion provide the self. }\end{array}$ & $\begin{array}{l}\text { humble, accepting my portion in life, } \\
\text { devout, respect for tradition, moderate }\end{array}$ \\
\hline $\begin{array}{l}\text { SECURITY } \\
(\mathrm{SE}):\end{array}$ & $\begin{array}{l}\text { Safety, harmony, and stability of society, } \\
\text { of relationships, and of self. }\end{array}$ & $\begin{array}{l}\text { family security, national security, social } \\
\text { order, clean, reciprocation of favours }\end{array}$ \\
\hline $\begin{array}{l}\text { POWER } \\
(\mathrm{PO}):\end{array}$ & $\begin{array}{l}\text { Social status and prestige, control or } \\
\text { dominance over people and resources. }\end{array}$ & $\begin{array}{l}\text { social power, authority, wealth, social } \\
\text { recognition }\end{array}$ \\
\hline $\begin{array}{l}\text { ACHIEVEMENT } \\
\text { (AC): }\end{array}$ & $\begin{array}{l}\text { Personal success through demonstrating } \\
\text { competence according to social standards. }\end{array}$ & successful, capable, ambitious, influential \\
\hline $\begin{array}{l}\text { HEDONISM } \\
(\mathrm{HE}):\end{array}$ & $\begin{array}{l}\text { Pleasure and sensuous gratification for } \\
\text { oneself. }\end{array}$ & pleasure, enjoying life \\
\hline $\begin{array}{l}\text { STIMULATION } \\
(\mathrm{ST}):\end{array}$ & Excitement, novelty, and challenge in life. & daring, a varied life, an exciting life \\
\hline $\begin{array}{l}\text { SELF-DIRECTION } \\
(\mathrm{SD}):\end{array}$ & $\begin{array}{l}\text { Independent thought and action-choosing, } \\
\text { creating, exploring. }\end{array}$ & $\begin{array}{l}\text { creativity, freedom, independent, curious, } \\
\text { choosing own goals }\end{array}$ \\
\hline
\end{tabular}

Note. Adapted from Schwartz \& Sagiv (1995, p. 95).

For less refined distinctions among values, it is also possible to use the four higher order value orientations (for example, Bernard, et al., 2006; Verkasalo, Lönnqvist, Lipsanen, \& Helkama, 2009). A score for conservation is computed by the mean of conformity, security and tradition items, for self-transcendence by the mean of benevolence and universalism items, for self-enhancement by averaging power and achievement items and for openness-to-change by averaging self-direction, stimulation and hedonism items (see also Schwartz, 2003). Like the ten basic value types, the higher 
order value orientations should exhibit a sinusoidal trend when correlated to other relevant variables.

Benefits of the SVS. There are a number of benefits of Schwartz' theory and value measure. The theory is explicit in the hypothetical relationship between value types and other relevant variables, and the empirical approach is therefore theoretical and not exploratory which renders the survey more advanced compared to a number of other value measures (e.g. the Rokeach Value Survey, Rokeach, 1973). Through the predictions about motivational conflicts and compatibilities between values, the model provides a single nomological net which allows it to merge diverse research on values across cultures (Pakizeh, Gebauer, \& Maio, 2007). Pakizeh et al. (2007) argue that because of this characteristic, the SVS is the most important recent development in research on values.

Schwartz (1992; Schwartz \& Sagiv, 1995) also pointed out that the theory includes a comprehensive set of values. No major value dimensions seem to be left out. The SVS is highly suitable for cross-cultural research: so far it has been translated into 47 languages using rigorous translation methods (Schwartz, 2006a). Data have been gathered from more than 70 nations including university students, secondary teachers and near-representative samples. Cross-cultural equivalence of 45 value items has also been established (Schwartz, 1992; Schwartz \& Boehnke, 2004; Schwartz \& Sagiv, 1995; Spini, 2003). The survey shows sound psychometric properties with satisfying reliabilities ranging from .61 (tradition) to .75 (universalism) when averaging across cultural samples (Schwartz, 2005b). Because of these benefits, Schwartz' theory and also his survey for the assessment of values were used in this thesis. 
The Culture-level

The Theory. Schwartz (1994b; 2006b) also developed a Culture-level Value Theory. Similarly to the individual-level, he postulated that there are basic issues in every society for which a solution must be found: (1) to what extent a person is embedded into a group, (2) how to preserve the social fabric, and (3) how to relate to the natural and social world. The solutions take the form of three bipolar value orientations: Autonomy (with the subtypes Intellectual and Affective Autonomy) versus Embeddedness; Hierarchy versus Egalitarianism; and Harmony versus Mastery. An Autonomy value orientation encourages individuals to pursue positive experiences for themselves (Affective Autonomy) as well as their own ideas and intellectual aspirations (Intellectual Autonomy). The opposite pole Embeddedness becomes more important in societies in which social relationships, the traditional order, and the maintenance of the status quo are seen as priorities. The second solution is either characterized by a Hierarchy orientation which emphasizes the legitimacy of a hierarchical social order and unequal resource allocation, or by Egalitarianism which emphasizes that all individuals of a given society are equal and mutual concern, as well as cooperation, is necessary to ensure everyone's welfare. The third societal issue is solved by a Harmony orientation which is accentuated in societies where the social and natural world is accepted as it is and emphasis is laid on fitting in harmoniously, or by a Mastery orientation which means to actively control the social and natural environment through self-assertion values. Cultural differences on these value dimensions emerge because culture has an influence on what pole of the orientation is emphasized in a society. The value dimensions again form a hypothetical circumplex structure which is depicted in Figure 1.2. Like the individual-level value structure, adjacent values signify compatibility and opposite values are in conflict with each other. 


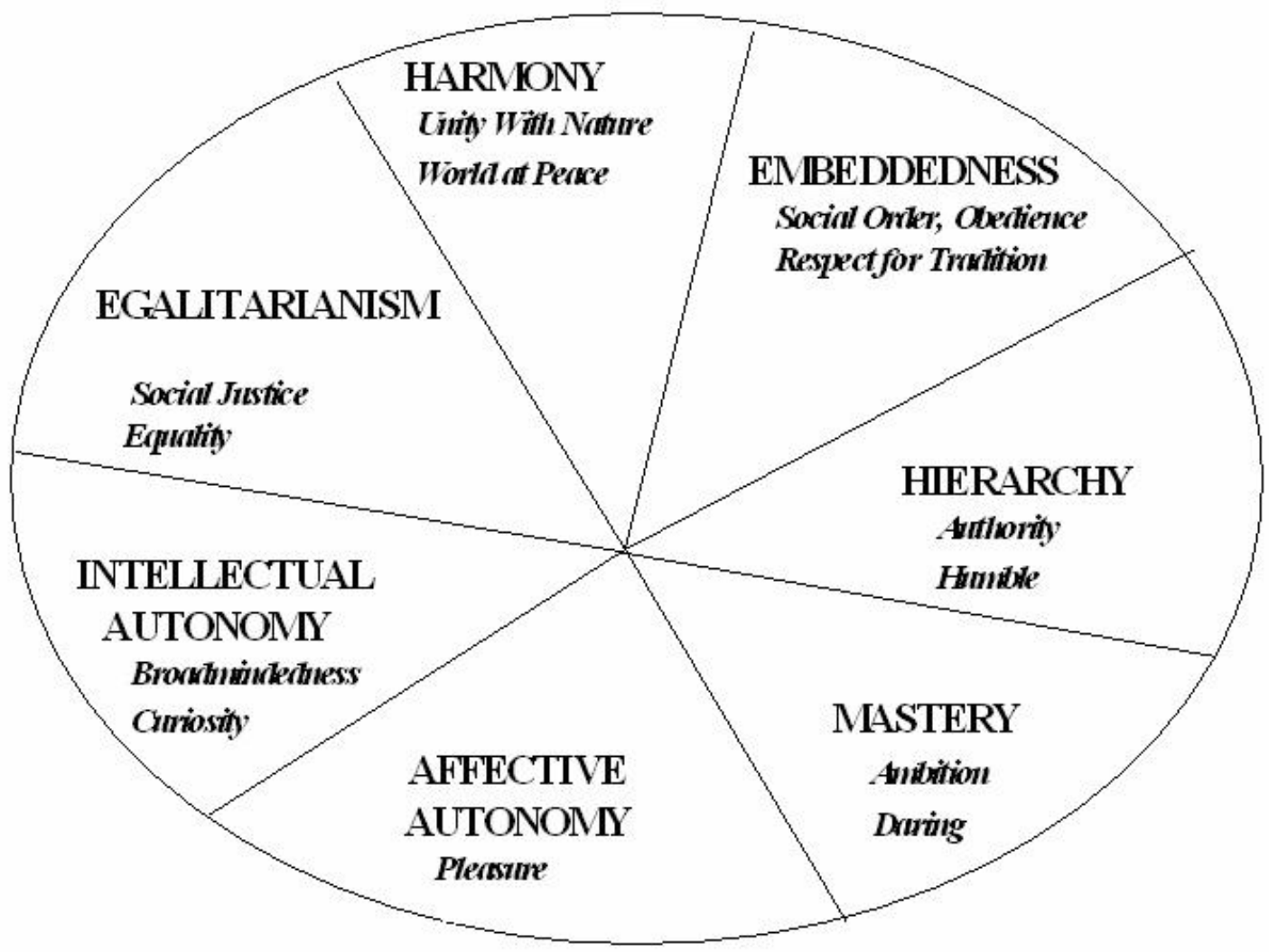

Figure 1.2: Circular model of relations among seven value dimensions at the culturelevel (from Schwartz, 2006, p. 142).

Note. Values in italics are example items for the respective value orientation.

The Empirical Test. Schwartz $(1994,2006)$ investigated the culture-level value structure by analyzing the same data as for his individual-level study (i.e. personal values), but this time at the aggregated culture-level. He used only the 45 value items that proved to be cross-culturally equivalent in meaning, and aggregated the data at the nation-level so that for each of the 45 value items a single average country score was available. Hence, any subsequent analysis was not based on the number of individual cases, but on the number of countries. He then examined the hypothesized value structure with smallest space analysis - initially with 38 nations (Schwartz, 1994) and later with 67 nations (Schwartz, 2006) - and was able to corroborate his theory. The culture-level value dimensions are computed by (1) aggregating the dataset at the 
culture-level so that for each value item there is a 'nation' score and then by (2) averaging the scores for value items that fall into the respective value cluster as depicted in Figure 1.2. The culture-level value types have been shown to relate meaningfully to a number of nation-level indicators, such as socio-economic development as well as political and population characteristics (Schwartz, 2006).

Benefits of the Cultural Values. Schwartz' cultural values dimensions belong to the broad frameworks that have advanced research in cross-cultural psychology (Smith, et al., 2002). Like Hofstede (1980), Schwartz $(1994,2006)$ has provided a map of cultures not based on geography, but on aggregated psychological data in terms of values. This has benefited cross-cultural research in two ways: First, with the help of this 'map' a systematic sampling could be employed in which cultures were selected in a systematic, theory-guided fashion to investigate cultural differences (cf. van de Vijver \& Leung, 1997). Secondly, it has become possible to 'unpackage' culture, i.e. to use values as a measure of culture and to investigate their effect on the variables of interest, instead of assuming cultural differences on the basis of prior cross-cultural research (cf. Kağıtçıbaşı, 1997; van de Vijver \& Leung, 1997). Both strategies will be employed in this thesis (systematic sampling in Study 3 and 4 and unpackaging of culture with the help of a multilevel model in Study 2).

Most studies have focused on individualism and collectivism which has initially been identified by Hofstede (1980). Individualism/ collectivism is still the most important dimension in cross-cultural theory and research (Matsumoto \& Yoo, 2006). It has been proposed that the fundamental difference between individualistic and collectivistic cultures lies in the way well-being is attained (see Oyserman, Coon, \& Kemmelmeier, 2002). Personal goals, happiness and personal control are central to the well-being of individuals in individualistic societies, while carrying out duties and obligations are central to the well-being in collectivistic societies. This bipolar value 
dimension is also present in Schwartz' Value Theory yet under a different name Schwartz' Autonomy versus Embeddedness dimension parallels the individualismcollectivism distinction (see also Gouveia \& Ros, 2000; Inglehart, 2006; Kağıtçıbaşı, 1997).

The Critique. While Schwartz has made an indelible contribution to the study of values, and the SVS is justifiably popular, it is not without its detractors. The main critique put forward in this thesis is that Schwartz' (1994) operationalization of cultural values does not assess the 'ought' character of desirable values in terms of a separate intrapsychic value system. The main drawback is one of 'circularity' which has already been identified as a problem for the construct individualism/ collectivism (Berry, 1992, as cited in Kağıtçıbaşı, 1997). Circularity is here a lack of independent measurement at the two levels of analysis (the individual- and the culture-level) combined with the use of correlations between them. This impedes a deeper understanding of the relation between the cultural context and the individual in research areas such as value change. According to Roe and Ester (1999), for example, it is difficult to conceptualize how a change in societal values may influence individuals' values if the societal values consist of the sum of all individuals' values. Hence, the operationalization of cultural values as the 'desirable' and as an independent macro-level construct is needed. This issue will be addressed in more detail in chapter two and empirically followed up in Study 3 and 4. 
Cross-cultural research on values is carried out on two different levels of analysis: the individual- and the culture-level (Smith \& Schwartz, 1997). As has been explained above, the two levels of analysis produce somewhat different value structures and some value items cluster differently across levels. Examples are the value authority and humble. At the individual-level, they are negatively correlated which means that individuals who see authority as a guiding principle in their life tend to reject being humble as their guiding principle (Smith, 2002). However, at the culture-level both values are positively correlated with each other, which means that nations that emphasize authority also show a preference for humility as a value. Hence, researchers have been advised to analyze their data at the individual-level if their interest lies in examining individual differences in values, and to analyze the data at the culture-level if their interest lies in comparing the values of different cultural groups (Smith \& Schwartz, 1997). Equating the culture-level with the individual-level, e.g. by claiming that individuals show an orientation towards Autonomy or Hierarchy, would mean committing the "ecological fallacy" (Hofstede, 1980, p. 29). The "reverse ecological fallacy" (Hofstede, 1980, p. 29) is committed when individuals' value types are taken to describe cultures.

Despite the compelling example from the Schwartz Value Survey corroborating the difference between the individual- and culture-level value structure, it seems that culture-level factors are not easy to understand, especially for psychologists who are used to interpret and operate with psychological phenomena at the individual-level (Bond \& Pang, 1991). Many writers in cross-cultural psychology have committed the ecological fallacy when they referred to individualism/ collectivism as a characteristic of individuals (Kağıtçıbaşı, 1997; Smith, 2002). As Kagitcibasi (1997) puts it: "The discrepancies between culture-level and individual-level constructs do not make 
immediate psychological sense and are confusing" (p. 16), even though it is statistically clear that a country-level analysis uses country scores and an individual-level analysis uses individuals' scores. This confusion is mainly based on one vexing question: "How can it be that when the same data are analyzed at two different levels, the results are not the same?” (Smith, Bond, \& Kağıtçıbaşı, 2006, p. 41). Since in the case of value measures the country scores are derived from averaging responses of a sample of individuals, the individual- and culture-level could be expected to have a parallel psychological meaning (Berry, 1992 as cited in Kağıtçıbaşı, 1997). However, with different value labels for dimensions at the culture- and individual-level, it is difficult to make sense of the culture-level dimensions. Kağıtçıaşı (1997, p. 33) even suggests that we may be dealing with a statistical artefact, instead of a true fallacy.

The issue is not only one of conceptual confusion, but also of methodological restriction: Value theories that can only be applied to certain levels impede, for example, a direct comparison of the predictive power of values at the individual- and the culture-level in multilevel models. For instance, Schwartz' (1992) openness-tochange versus conservation dimension has been compared with Hofstede's concept of individualism and collectivism (Kağıtçıbaşı, 1997). The question whether openness-tochange predicts criterion variables in a similar fashion at both the individual- and culture-level would be interesting to examine. Imagine that openness-to-change at the individual-level is a significant predictor of tolerance towards homosexuality. It would be interesting to know whether openness-to-change at the culture-level would also significantly predict individuals' tolerance towards homosexuality. Moreover, the question could be examined whether the same kind of values would add any additional explained variance at the culture-level if individual differences in value priorities are controlled for. This would provide a new insight into the effect of culture on 
individuals' attitudes. This question, however, cannot be investigated if value orientations are not equivalent at both levels of analysis.

Schwartz (1994) himself has pointed out that it is reasonable to assume that there is some structural isomorphism between values at the individual- and the culturelevel. However, until very recently it has never been studied how much overlap there is indeed between the two levels of analysis (cf. Matsumoto, 2003). Fischer, Vauclair, Fontaine and Schwartz (in press) directly examined the degree of similarity between the structure of values at the individual- and culture-level using recent data from the Schwartz Value Survey drawn from more than 50 countries. The findings suggest that Schwartz' individual- and culture-level value structures are very similar to each other and that a conceptual separation of value orientations according to the level of analysis is possibly not justified. Even though the authors did not find perfect isomorphism, they found a substantial similarity in structure across levels. Fischer and Poortinga (2009) also examined isomorphism of value structures across levels of analyses. They compared personal, social (ratings of other people's values) and cultural value systems as assessed with the SVS. They found that the value structures were highly similar which led to the conclusion that a single value structure is most parsimonious. There was little empirical evidence that would suggest that the individual- and culture-level value structure were different from each other to the extent that they needed to be treated as distinct. The authors stated that using the same individual-level value clusters at both levels of analysis would simplify value research without a substantive loss of information. This provides an empirical justification for a practice that has already been implemented in some studies (see e.g. Lönnqvist, et al., 2009; Verkasalo, et al., 2009). 


\section{Conclusion}

This chapter introduced the most relevant theoretical frameworks on morality and clarified in which framework this thesis is positioned. It has outlined the two distinct research streams of morality and social psychological values research that come together in the concept of moral values. I have pointed out that there is a gap of research in investigating the social psychological concept of values, as terminal or instrumental goals, in terms of their moral relevance to individuals. I have also indicated that the assessment of moral values may be a valid and necessary alternative to measuring cultural values through the statistical aggregation of personal importance ratings of values.

In the next section, I shall examine more thoroughly the issue of inferring culture from personal importance ratings of values. This theoretical chapter is aimed at addressing the following questions: What is culture? What does it mean if culture is measured by aggregating personal values? What kind of conceptual and methodological issues are associated with this practice? Is this a real problem or, in other words, is there empirical evidence that shows that it posits indeed a problem to the understanding of culture? This literature review will be followed by a proposal to measure culture through the assessment of moral values. Chapter two ends with a value taxonomy in which the different kinds of values (e.g. personal values, moral values) can be categorized and recent empirical research can be fitted in. 


\section{Measuring Cultural Values:}

\section{Considering Morality in Cross-cultural Value Research}

\section{Introduction}

Research in cross-cultural psychology has investigated culture by focusing mainly on cognitive systems comprising different concepts such as values, attitudes, and beliefs. Most of the research has dealt with the concept of values to understand culture (e.g. Bond, et al., 2004; Earley, 2006). This is probably due to the common understanding in the field that values are "among the building blocks of culture" (Hofstede, 1980, p. 25). More recently, cross-cultural researchers have raised doubts about the usefulness of the value concept (Gelfand, et al., 2006). Empirical findings show mixed results with some studies supporting the explanatory power of values and other studies showing that values are not sufficient for understanding cultural differences (see Gelfand, et al., 2006). Furthermore, there is growing scepticism at a theoretical level that the mere cognitive approach to culture, where cultural values are detached from contextual processes such as social constraints and norms, is useful to understand culture (Earley \& Mosakowski, 2002; Gabrenya, 1999; Mogghaddam \& Studer, 1997). It is not surprising that some researchers have suggested abandoning the value concept and have turned to potentially more useful concepts such as social axioms (Leung, et al., 2002) or 'cultural intelligence' (Earley \& Ang, 2003).

Note. An earlier version of this chapter has been published and is hereafter referenced as follows: Vauclair, C.-M. (2009). Measuring cultural values at the individual-level: Considering morality in crosscultural value research. Mackenzie Business Review. Special Issue: Human Values - Theory and Practices, 10(3), 60-83. 
The scepticism may be legitimate to the extent that most cross-cultural researchers define cultural values as a conception of 'the desirable' (Braithwaite \& Scott, 1991), by describing them as "desirable transsituational goals" (Feather, 1996; Kluckhohn, 1952; Schwartz, 1994b, p. 21), but surprisingly take a rather asocial or acultural stance, when measuring values through personal importance ratings of individuals. This approach assesses values as 'the desired' or in other words what people personally want in their lives. In contrast to that, values as 'the desirable' target people's opinion of what they 'ought' or 'should' strive for, which is influenced by culture and implies social constraints and pressures imposed upon the individual (see also Hofstede, 1980; Higgins, 2006; Kluckhohn, 1952; Lewin, 1952). The purpose of this chapter is to highlight this distinction and to stress the importance of social constraints and normative pressures for the measurement and understanding of culture if culture is defined as shared values and beliefs. With the help of a value taxonomy, past research is classified regarding their focus of measurement. Within this taxonomy suggestions are made how it may be possible to measure values as 'the desirable'. Finally, the benefits of distinguishing these different kinds of values are discussed in regard to future research.

\section{Definition of Culture}

While culture can be defined in many different ways (cf. Baldwin, et al., 2006; Kroeber \& Kluckhohn, 1952), there seems to be a minimal consensus - at least in the field of anthropology and psychology - that it is a phenomenon of a collective and it is shared among its members (Kashima, 2000; Kuper, 1999; Lehman, Chiu, \& Schaller, 2004; Rohner, 1984). Yet, there seems to be no agreement of what exactly this 'sharedness' means (Rohner, 1984). Some cross-cultural researchers have conceptualized it in terms of observable patterns of cognitive structures which are 
distinctive to social groups (e.g. Hofstede, 1980). These are concepts such as values and beliefs that are assumed to influence behaviour. This places culture inside the minds of individuals as members of a cultural group, like in Hofstede's definition of culture as "the collective programming of the mind" (Hofstede, 1980, p. 13). Other definitions of culture in cross-cultural psychology go beyond the structural view by including the functions of culture (e.g. Matsumoto, 2007). Functional definitions suggest that concepts such as cultural values exist for a reason or purpose. One of the most important functions of culture is possibly its "guidance function" (cf. Baldwin, et al., 2006, p. 38; cf. Kluckhohn \& Strodtbeck, 1961): Culture helps to organize collective life by providing solutions to problems of everyday activities. It guides individuals by reducing the number of available interpretations of an event or person and in this way it is giving order to the world. It is this sense of order that people transmit via cultural values to new cultural members, which can be employees in an organization, migrants in a host country, or growing children (Baldwin, et al, 2006; Roe \& Ester, 1999). Individuals are constrained by culture in so far that to be accepted as group members, they need to conform to cultural standards, which reinforce learning and internalization of cultural values (see also Triandis, 1972). This kind of structural-functional understanding of culture has been widely adopted by current cross-cultural theorists (e.g. Schwartz, 1994, 2006b; Triandis, 1994).

From this point of view, shared values serve a purpose in social groups or cultures. Shared values are general guidelines which regulate behaviour of its members so that collective life is organized and individuals interact more smoothly. New members are explicitly and implicitly taught what is appropriate or socially desirable. Thus, culture draws boundaries and limits the individual's freedom by creating external constraints on what is accepted or appropriate and what is not. In this way, cultural values refer to what is judged as right or wrong, i.e. they refer to issues of morality. In 
fact, it has been proposed that it is difficult to delineate where culture ends and morality begins as culture and morality share an intricate and intimate relationship (Miller, 2001). Yet, this close interrelationship between culture and morality has so far been neglected in quantitative research on cultural values.

In the following section the usual approach in identifying cultural values is briefly outlined as well as the methodological and conceptual problems associated with it. Subsequently, selected empirical studies are presented to corroborate the raised concerns of neglecting morality in form of social constraints in cultural values research. Recent alternative approaches of measuring cultural values are introduced and classified in a value taxonomy. A possible solution for measuring the so far neglected moral component of cultural values at the individual-level is finally presented.

\section{Past Approach in Identifying Cultural Values}

\section{The Culture-level Analysis Approach}

Cross-cultural research on values is carried out on two different levels of analysis: the individual- and the cultural-level (Smith \& Schwartz, 1997). At the individual-level, values are conceptualized as motivational goals serving as guiding principles in individuals' lives (Rokeach, 1973; Schwartz \& Bilsky, 1987, 1990). The procedure consists usually in asking individuals from different countries to respond to the same value survey and to indicate which values are personally important to them. After establishing equivalence of the scale, structure-oriented techniques, such as factor analysis or multidimensional scaling, can be employed to identify value dimensions that characterize individuals in their motivational goals. Schwartz (1992), for example, found that values are ordered in a circular fashion with the consequence that if individuals place emphasis on one value type (e.g. hedonistic values), they are likely to deemphasize the opposite value type on the value circle (here traditional values). Yet, 
these value dimensions do not represent cultural values, but just personal values. To measure cultural values, the responses of individuals need to be taken a step further and analyzed at the cultural-level. Here, the scores of individuals within countries are averaged, meaning that the culture-level dataset consists of countries as cases instead of individuals. These country scores can then also be analyzed with structure-oriented techniques to identify cultural value dimensions. Schwartz $(1992,1994)$, for example, found that a culture-level analysis of his cross-cultural data yielded also a circular value structure. Only that this time the value dimensions characterize whole societies and are different in their content compared to the individual-level dimensions. Countries that emphasize one value type (e.g. Egalitarianism) deemphasize the opposite value type in the value circle (here Hierarchy). These values are seen as representing the socially shared ideas of what is desirable or good and right in a society (cf. Smith \& Schwartz, 1997). They characterize cultures and are therefore labelled "cultural values".

\section{Conceptual and Methodological Problems}

As already indicated above, the concept of values is multifaceted in the sense that it can describe not only individuals, but also cultural groups. Yet, since Hofstede (1980) it is common knowledge that what applies to cultural groups does not necessarily apply in the same way to individuals. Hofstede (1980) found that his country-level dimensions, such as collectivism and individualism, could not be replicated at the individual-level. Since then, the two levels of analysis are neatly separated, and applying country-level dimensions to individuals is seen as committing the "ecological fallacy" (cf. Hofstede, 1980, p. 28-31). The consequence of this fallacy problem is, that in a strict sense one cannot compare individuals in terms of cultural value dimensions such as individualism and collectivism. This is a characteristic that applies only to countries, cultures or other social entities. From a psychological perspective, the 
ecological fallacy remains a puzzling and less well-understood phenomenon until today (Smith \& Schwartz, 1997). After all, the country-level dimensions are derived from individuals' responses. Yet, statistically aggregating their responses at the country-level renders them inapplicable to individuals. Another puzzling issue is that, only the culture-level approach is seen as measuring culture in the sense of what is socially shared and the abstract ideas of what is good, right, appropriate and desirable in a cultural group (Hofstede, 1980; Schwartz, 1994; Smith \& Schwartz, 1997). An inevitable conclusion is that if we want to study cultural values, we need to 'limit' ourselves to country-level analyses. However, there are several objections to this inference.

The first objection is epistemological in nature referring to the assumption that only country-level dimensions reflect shared cultural values. In country-level analyses, culture is treated as the sum of all sampled individuals from a specific cultural group. That means that culture is seen as only measurable when the responses of individuals within a cultural group are aggregated. This may be adequate for sociological endeavours, also reflected by the usual procedure of validating country-level dimensions with sociological indices such as gross domestic product (e.g. Hofstede, 1980). However, from a psychological perspective of culture, with the aim to understand human behaviour, it seems more relevant to try to comprehend each human being as an expression of culture (Bruner, 1990), and thus to investigate the manifestation of cultural values at the individual-level relying on intrapsychic cognitive structures of individuals. A possible way of doing this could be by including morality as a concept in the measurement of values to capture external normative pressures in a society. This will be discussed in more detail below.

A further objection concerns the way cultural values are operationalized. Most of the value surveys measure the personal importance of values (e.g. Chinese Value 
Survey, Chinese Culture Connection, 1987; Rokeach Value Survey, Rokeach, 1973; Schwartz Value Survey, Schwartz, 1992). The Schwartz Value Survey (SVS) (Schwartz, 1992), for example, requires individuals to indicate "What values are important to ME as guiding principles in MY life?" From a social psychological point of view, the statistical average of personal value priorities does not tell us what kind of social constraints or normative pressures are prevalent in a certain culture. It does not answer questions such as: What are we supposed to value or strive for? Or what kind of behaviour are we supposed to adopt in different cultures? Averaging personal value priorities reflects merely what a majority deems as important in a culture and can be called "actual rather than ideal cultural value priorities" (Schwartz, 1992, p. 51). The problem is that operationalizing values with personal importance ratings conflates two different motives of valuing: values may be seen as personally important, because (1) they are well internalized socially desirable values, or (2) they have an intrinsically positive quality which leads to a hedonic experience of appraisal when the value is fulfilled (see also Feather, 1999; Higgins, 2006). For instance, the value 'pleasure' from the SVS may be judged as important because of this very hedonic motive and not because it is a socially desirable standard. Values as 'the desirable' go beyond the evaluation of whether a value is just pleasant or unpleasant for oneself by implying judgments of what is desirable or morally right or wrong. The usual procedure of averaging personal values and deriving cultural values from that neglects these kinds of social constraints and expectations that culture imposes upon individuals.

The last objection deals with the methodological consequences. Sticking to the culture-level approach to identify cultural values restricts future research in a practical sense: either we limit ourselves to cultural value dimensions that have already been identified, for example, the seven culture-level value types by Schwartz (1994), or every researcher who suggests potentially new and meaningful values (e.g. 'protestant ethic 
values', Verkasalo, et al., 2008) needs to conduct an extensive cross-cultural study to be able to claim that they are valid cultural values. This would mean sampling of individuals from at least 50 countries or cultural groups to ensure that the new culturelevel value dimension is stable (Fischer, et al., in press). It would also mean conducting a very costly, effort-and time-intensive research project (cf. Norenzayan \& Heine, 2005).

\section{Empirical Evidence Underpinning the Problems}

One may wonder whether personal and cultural values are indeed different. Or whether it would really make a difference if cultural values were not derived from a culture-level analysis, but measured at the individual-level and differently from personal values. Would it really add something to our understanding? There is indirect empirical evidence that it would. It seems indeed that personal importance ratings of values do not capture the normative aspect of values prevalent in social groups.

As Fischer (2008a) pointed out, if culture is defined in terms of shared values, any measure of cultural values should show that individuals from the same culture endorse similar values, and that cross-national variation exceeds indeed intranational variability. Cross-cultural data from the Schwartz Value Survey study analyzed at the individual-level revealed that there is "substantial individual variance in response to every single value" (Schwartz, 1992, p. 50) instead of consensus which would have been an indicator for shared cultural values. Furthermore, this individual variance does not seem to reflect measurement error as it was systematically associated with individual differences in participants' background characteristics, their attitudes or behaviour. Schwartz eventually concludes that his survey is measuring personal values and that "one cannot derive the normative ideals of a culture from the average of individual responses" (p. 51). 
Furthermore, it seems that personal values can even be compromised when the normative pressure is high. Bardi and Schwartz (2003) conducted a study to investigate the value-behaviour link at the individual-level. They assessed values with the SVS and sampled behavioural items supposed to represent the values. Their results showed that the more frequent a behaviour was reported, the less consistently values were associated with the behaviour. In other words, if everybody seems to behave in a certain way or if a certain behaviour is the norm in a social group, people report to behave the same way even if it is not congruent with their personal values. Only when external pressure is absent, the personal importance of values may influence their behaviour. Since from a functional perspective culture can be seen as creating external constraints and putting normative pressures on the individual, Bardi and Schwartz' study underpins the here proposed argument that personal importance ratings may not reflect the 'shared' ideas in a social group what one should do or should strive for. And from a conceptual perspective this does not change if the data is analyzed at a statistically higher level.

Another area of research in which the conceptual and empirical distinction of personal values and those of the social context may play an important role, is in the area of value fit between individual and social group as well as value change. Rohan and Maiden (2000) used a modified form of the SVS to measure value priorities of teachers and what they call the 'ideological' value system of their schools which is the teachers' perception of the school's value priorities. They showed that the fit between teachers' personal values and their perception of the school's values strongly predicted reported stress, job commitment, and satisfaction. As Rohan and Maiden state, this prediction could not have been obtained by merely constructing an index of fit based on teachers' personal values and the aggregated value priorities of all teachers in the respective schools. The fundamental issue lies in the fact that aggregated personal values of individuals do not contain much social psychological information. 
If value change is investigated, the importance and benefit of operationalizing values of the social environment and of the individual differently becomes even more obvious. There is the problem of causal inconsistency if cultural values are represented by mere aggregation of individuals' personal values. As Roe and Ester (1999) highlight: "It is hard to assume that a change in cultural values causes a change in individual values if the change in cultural values is operationally defined as the sum of individual changes" (p. 4).

These empirical studies and examples suggest that personal value priorities (1) do not contain information about values that are socially desirable, and (2) impede a deeper social psychological understanding if they are not operationalized separately for the micro-and macro-level. The following section proposes how values can be operationalized at these two conceptually different levels.

\section{Alternative Ways of Measuring Cultural Values \\ Recent Approaches: Changing the Frame of Reference}

Recent studies have adopted the approach to measure cultural values by changing the frame of reference from 'self' to 'others'. Here individuals are asked about their perception of other people's values (Fischer, 2006; Wan, Chiu, Peng, et al., 2007; Wan, Chiu, Tam, et al., 2007). For instance, Wan et al.'s (2007) underlying assumption is that cultural values are those that are strongly endorsed by most members of the culture. Instead of just averaging the value priorities of a social group, they argue that it is more appropriate to let research participants estimate how an average member of a cultural group would respond to the value survey as culture resides in its members' intersubjectivity. Consequently, a value has high intersubjective importance when most of the research participants agree that an average member of their group would strongly endorse the value. Wan and colleagues used the Schwartz Value Survey (SVS) and 
measured personal values of Chinese university students. They also asked the participants in a modified version of the SVS to rate the importance of the values to 'an average student at the university' and to an average person of their cultural group. They called this measurement strategy 'intersubjective consensus approach' or 'perceived cultural importance' approach alternatively. They used this approach to identify those cultural values that differentiate between Chinese, Singaporean and the local university culture. Their findings showed a general tendency, namely, that the more personally important 'culturally' distinctive values were rated, the more respondents identified with the respective cultural groups suggesting that personal values may reflect cultural identity and thus cultural values. However, as the authors note this relationship did not hold for all cultural identifications, but only for those referring to subcultures, for instance, identification with the university culture. The reason may be that it is difficult for respondents to rate the value importance of an average person belonging to a greater cultural entity such as national culture.

Fischer's (2006) solution for measuring cultural values also consisted in changing the frame of reference. The SVS was taken for this purpose and modified by instructing respondents from ten different countries to rate the importance of each value for most of the people in their country of birth. He analyzed the data at the culture-level and correlated them with Schwartz' culture-level data on self-referenced ratings of values. The results illustrated that self- and culture-referenced ratings did not show great overlap, sharing only $7.84 \%$ of their variance. Only values of the cultural value dimensions embeddedness (feeling attached and socially connected to social groups) and affective autonomy (pursuing affectively positive experiences for oneself) were related to self-referenced ratings. Fischer concluded that researchers need to be careful about interpreting aggregated self-reports in terms of cultural values and that there is 
more research needed that investigates the normative aspects of culture to understand the functions and processes of culture.

These value studies have contributed to an advancement in cross-cultural value research by considering different references when using self-reported ratings of individuals. To provide a better overview, a value taxonomy is presented where these studies can be classified.

\section{Classifying Value Research at the Individual-level: A Value Taxonomy}

Table 2.1 shows how past research investigating values at the individual-level may be classified. There are two dichotomous features of values: they can either refer to the individual or collective and to 'the desired' (what and individual wants as a goal in life) or 'the desirable' (what an individual ought to want as a goal in life). This yields a 2 X 2 classification of four different value types. Personal values refer to the individual and what they desire for themselves which has, for example, been studied by Schwartz (1992). Studies that change the frame of reference to value importance ratings of a social group measure conceptually also what is desired (cf. Fischer, 2006; Wan, et al., 2007). However, here it does not apply to an individual, but to a whole social group. These kinds of values may be labelled 'social value system' (Rohan, 2000). The term does not refer to the content of values, for instance, prosocial, desirable or moral values, but to the holder of these values, which is a social entity. In order to assess the normative component of values in the sense of what one should or ought to strive for, values need to be measured as the desirable. Possible solutions to achieve this are proposed next. 
Table 2.1: Value taxonomy: The measurement of values at the individual-level

\begin{tabular}{lll}
\hline & \multicolumn{1}{c}{ Frame of Reference } \\
\cline { 2 - 3 } $\begin{array}{l}\text { Desired } \\
\text { (wanting) }\end{array}$ & \multicolumn{1}{c}{ Individual } & \multicolumn{1}{c}{ Collective } \\
& $\begin{array}{l}\text { Opersonal Value System } \\
\text { me/ you }\end{array}$ & Social Value System \\
\hline $\begin{array}{l}\text { Desirable } \\
\text { (ought) }\end{array}$ & $\begin{array}{l}\text { Personal Moral Value System } \\
\text { (personally desirable) }\end{array}$ & $\begin{array}{l}\text { Social Moral Value System } \\
\text { (socially desirable) } \\
\text { subculture) }\end{array}$ \\
& $\begin{array}{l}\text { Operationalization: What one culture (or specific } \\
\text { ought to strive for; right vs. } \\
\text { wrong; feeling of guilt if value } \\
\text { violated }\end{array}$ & $\begin{array}{l}\text { Operationalization: What one is } \\
\text { expected to strive for in the } \\
\text { res culture }\end{array}$ \\
\hline
\end{tabular}

Incorporating Morality in the Measurement of Values

The Difference between 'the Desired' and 'the Desirable'. While studies described above measure what is desired, cultural values may be better operationalized if measured as 'the desirable'. Values as 'the desired' refer to what individuals intrinsically 'want' to strive for or what they personally desire in their life (e.g. Braithwaite \& Scott, 1991). Value instruments that measure 'the desired' address individuals by instructing to ask themselves, for example, what values are important to them as guiding principles in their life (e.g. Schwartz, 1992, p. 50). In contrast to that, values as 'the desirable' refer to what individuals 'ought' to strive for or what they should desire (Dewey, 1939) which in regard to culture is something that has been extrinsically imposed upon them. An alternative way of conceptualizing the difference between 'the desirable' and 'the desired' is by assigning values 'direction and intensity' (Hofstede, 1980, p. 20; see also Higgins, 2006; Kluckhohn, 1952; Lewin, 1952). 'The desirable' represents the directional component of values and has an evaluative meaning, in the sense that individuals judge whether a certain value is right or wrong to 
strive for (cf. Kluckhohn, 1952). 'The desired' on the other hand refers to the strength or intensity of a value. Thus, holding a value means not only that this value is relevant or important to a certain degree (intensity), but at the same time it can also be evaluated in a sense of whether it is right or wrong to strive for it (direction). Hence, values as 'the desired' and 'the desirable' relate to values of two different natures. According to Hofstede (1980) this has implications for the interpretation of their norms. Taking the statistical average of values as 'the desired' indicates to what extent the majority endorses the values. This is an approach which has been mostly adopted in crosscultural value research (e.g. Chinese Culture Connection, 1989; Schwartz, 1994). In the case of 'the desirable', the assessed values contain implicitly an ideological norm in the sense of what is seen as morally ${ }^{2}$ right or wrong (cf. Hofstede, 1980; Kluckhohn, 1952). Hofstede (1980) provides a plausible example as an illustration of the difference between 'the desired' and 'the desirable': An individual may 'desire' "wealth" as a value or "having money" and consider it as highly relevant (intensity), in the sense that one needs some of it to lead a decent life. Thus, 'wealth' is seen as something important and its fulfilment may lead to a positive intrinsic feeling, such as satisfaction. However, the same person, especially if adhering to Christian principles, could judge this value as wrong in the sense of adopting it as 'a guiding principle in life'.

Yet, how can 'the desirable' component of values be measured? This is discussed next with regard to whether values refer to what an individual deems as desirable or to what a social group judges as desirable.

Social Moral Values: 'The Desirable' and the Collective. Similar to the operationalization of 'the desired' for a collective, the choice of reference may be that individuals are asked about other people in their culture and what kind of values others

\footnotetext{
${ }^{2}$ Hofstede (1980) does not use the term moral, but says that the ideological norm is "pertaining to what is ethically right" (p. 21). I use the term ethics and its derivations only to refer to philosophy as the science of ethical reasoning. When it is about people's everyday judgments of right and wrong, I assign it to the realm of morality.
} 
may regard as right to strive for. Values have been described as possessing a bipolar valence (e.g. Fishbein \& Ajzen, 1975; Rokeach, 1973). Thus, a possible way of measuring desirable values is to ask individuals whether other people in their society would judge certain values right or good as opposed to wrong and bad (see also Hofstede, 1980). The evaluation factor of the semantic differential developed by Osgood and colleagues (Osgood, Suci, \& Tannenbaum, 1957) may be very useful for this purpose. Osgood et al. (1957) found in an extensive cross-cultural study in more than 20 cultural groups that three distinctive factors emerge when bipolar adjectives are taken to assess different concepts: evaluation (e.g. good-bad), potency (e.g. strongweak), and activity (e.g. active-passive). The evaluative valence of values may be examined to find out which values are desirable or judged as right to strive for in a society. However, if questions are referred to other people, there is always the possibility that respondents simply do not know or are not aware of the values that others deem as desirable (see also Wan, et al., 2007). Another problem is that individuals may infer from their own values to those of others and regard them as normative, known as the false consensus effect in the social psychological literature (for a review see Marks \& Miller, 1987). A solution may be to rely on intrapsychic cognitive structures which are based on individuals' own experience. This could be done, for instance, by asking what kind of social expectations individuals experience in a particular social group or society. A possible question to measure socially desirable values is then, for instance, "to what extent do you think you are expected (or supposed) to follow this value in your society/ culture etc.?" Since internalization of cultural values is a lifelong process (cf. Allport, 1955) where individuals try to reconcile social constraints with their personal desires (see also Deci \& Ryan, 1995; Rohan \& Zanna, 1998; Zavalloni, 1980), the kind of social expectations that are imposed upon 
individuals may be psychologically very well accessible. To the best of my knowledge, there are no cross-cultural studies measuring this aspect of values.

Personal Moral Values: 'The Desirable' and the Individual. Assessing what is desirable for an individual gives moral values another spin. What is important here is that values are not only imposed, but are also internalized by the individual to a certain degree (cf. Ryan \& Deci, 2000). How can these kinds of values be measured? One could again use a bipolar evaluative response scale of 'good vs. bad' or 'right vs. wrong' (cf. Osgood, Suci \& Tannenbaum, 1957), but this time the choice of reference would be the individual in the sense of what she/he regards as right or wrong to strive for in life. The average of respondents' answers represents then not only a statistical norm in a cultural group, but also a deontological one (pertaining to what one ought to strive for). Following this procedure, a researcher may be able to ask simultaneously for each value the personal importance (important-unimportant) and its evaluative meaning. Thus, it would be possible to examine the congruence between what people 'want' to strive for in life and what they think they 'should want' to strive for as a guiding principle in life.

The drawback of this method could be that individuals may not assign 'deeper' meaning to the evaluative scale and rate what is 'important' and what is 'right' in the same way, possibly as an attempt to reduce cognitive dissonance (Festinger, 1957). Another possibility of measuring 'the desirable', which may be more reliable as it is less subjected to response styles, is to ask for moral emotions such as the feeling of guilt when violating a value. Moral emotions are in general seen as an important motivational factor to do good and avoid doing bad (Bedford \& Hwang, 2003; Tangney, 2002). It is the self-conscious emotion of guilt that plays a major role in morality (Amodio, Devine, \& Harmon-Jones, 2007; Baumeister, Stillwell, \& Heatherton, 1994; Eisenberg, 2000; Haidt, 2003). Guilt is widely recognized as an important self-punishing emotion for action evaluation and regulation (Fontaine, Poortinga, Setiadi, \& Markam, 2002). It is a 
negative affective experience that is evoked when one's behaviour falls short of either personal moral or societal standards (Amodio, et al., 2007). Shame is also a moral emotion, but associated with a rather egocentric concern for one's self-image. Guilt is more an allocentric concern for the consequences of one's behaviour on other people (Bedford \& Hwang, 2003; Tangey, 2002). As a consequence, moral transgressions have generally been considered to be more linked to guilt than to shame (Bedford \& Hwang, 2003). Thus, asking for guilt feelings when violating a value and examining its statistical average may be a good indicator for shared desirable and therefore cultural values that have been internalized.

\section{Benefits of Distinguishing Different Value Measurements}

Personally Desired versus Personally Desirable. The value taxonomy classifies value research into the dichotomous dimension of measuring 'the desired' aspect of values (through importance ratings) or the evaluative component of values in form of what is 'desirable'. Furthermore, it distinguishes whether values refer to the individual or a collective. Crossing these dimensions yield, for example, that values can be classified as 'personally desired' and 'personally desirable'. It may not be obvious to see the difference between these two kinds of values. This section deals with their fundamental distinction and how it may relate to the prediction of behaviour.

The problem with measuring values as 'the desired' through importance ratings is that there is a great deal of ambiguity regarding their underlying meaning. Values that individuals judge as important may have two different motivational sources: First, important values may be those that were initially extrinsic and have been imposed upon the individual by the social environment. Through socialization processes, the individual may have integrated it as part of the self, so that they become personally important (cf. Ryan \& Deci, 2000). The second motivational source is completely 
different representing an intrinsic kind of motive. Here, individuals may judge values as important, because fulfilling them leads to enjoyment or inherent satisfaction (see also Wan, et al., 2007; Ryan \& Deci, 2000). The problem is that assessing the personal importance of values does not allow distinguishing between these two different sources of motivation. As already outlined above in which the Bardi and Schwartz (2003) study has been referred to, it seems that this is not a trivial distinction. Bardi and Schwartz (2003) showed that personal values - which conflate the intrinsic and extrinsic dimension of values - were not related to behaviour when the normative pressure of the behaviour was high. It is possible that the value-behaviour link is more stable if not personally desired values are measured, but personally desirable values as they may provide an internal compass of 'oughts' and 'shoulds'.

Research on moral mandates may corroborate this assumption showing that personal moral values have a potential to influence people's behaviour (Skitka, 2002; Skitka \& Houston, 2001; Skitka \& Mullen, 2002). Skitka and colleagues showed that the value-behaviour link is especially strong for individuals with moral mandates, which are selective expressions of values and central to people's sense of personal identity. Moral mandates are characterized by attitude strength, importance, and certainty, but include the additional layer of moral conviction, i.e. a strong belief that an issue is right or wrong. People who develop moral mandates are highly motivated to express and protect them. Skitka and Mullen (2002) examined reactions of US-American adults to the Elian Gonzales case that became the centre of a heated public controversy and custody battle between his father in Cuba and his extended family in the USA. People in both the USA and Cuba engaged in demonstrations to defend their divergent position and values on the same issue. Skitka and colleagues research can be interpreted in the way that personally desirable values may yield strong links between values and behaviour. Hence, the reputation of values as being only distally related to behaviour 
may be eventually refuted when assessing values as the 'personally desirable' (cf. Hitlin \& Piliavin, 2004).

Individual versus collective - when is culture measured? Values may refer to the individual or a social entity. Socially desirable values as depicted in Table 2.1 have been discussed as measuring the values of the social environment such as the cultural group. Yet, personally desirable values may also be used to measure cultural values. If individuals are, for example, asked whether they would feel guilty after violating values, a high statistical average on certain values may indicate that these are the internalized shared values in the respective culture. Thus, both procedures may lead to the assessment of cultural values. However, they are fundamentally different in two ways: First, socially desirable values measure only the perceived values of a social group. This is adequate if operationalizing the cultural context, for example, is the focus of the study. Yet, it does not provide any information whether the respondents adhere to these values. Whether these values have been internalized or not may be important to assess in studies that focus on the relation of values to other variables, such as behaviour. As it has been outlined above, there may be a strong value-behaviour link if individuals' personal moral values are assessed. Secondly, if the focus of a study is on examining individual differences in personal moral values, assessing the perceived values of the social environment is inadequate.

The intriguing feature of moral values and the process of internalization is that they can stem from different sources located on a continuum of more proximal sources such as caregivers and peers to more distant sources such as teachers, media and society (Bandura, 1991). Therefore, internalized moral values may potentially reflect those of a culture. However, especially when it comes to strong political ideologies, it is also possible that individuals develop different internalized values, provided by their caregivers, for example, than those that are perceived as prevalent in the wider society 
and in the political propaganda. Prominent examples are the German siblings Sophie and Hans Scholl who during the third Reich in Germany opposed actively the national socialist regime and their supporters. They strongly supported the humanist values of their father. They regarded these values as the morally right ones and fought for it, taking into account that they may be and finally were sacrificing their own lives. The proposed value taxonomy takes this important aspect of moral values into account, i.e. the possibility that there are individual differences in moral value endorsement despite normative pressures.

\section{Conclusion and Final Remarks}

There has been a lot of definitional inconsistency in values-related theory and research (Rohan, 2000). Values have been used to refer to other psychological constructs such as attitudes, beliefs, norms, and behavioural self-reports (cf. Hitlin \& Piliavin, 2004) as well as interests, pleasures, likes, preferences, duties, moral obligations, desires, wants, goals, needs, and many other kinds of selective orientations (see Rohan, 2000). However, if values as 'the desirable' are the target concept to understand culture, it is important not to confound them with other related concepts. Each type of concept may play a distinctive role in explaining and understanding behaviour. This is why a value taxonomy has been proposed to clearly distinguish between individual and collective values as well as 'the desired' and 'the desirable'. While 'the desired' may be associated with a purely intrinsic motivation to pursue values, 'the desirable' contains also social psychological information, such as the internalization of extrinsic demands and behavioural regulation.

While some researchers argue to abandon the value concept (e.g., Gelfand, et al., 2006; Leung, et al., 2002), I see values as a useful concept to understand culture. The dissatisfaction with the value concept may rather be associated with the fact that value 
research has still not come to its full potential. Hopefully, the value taxonomy may inspire researchers to operationalize cultural values beyond mere importance ratings.

As De Munck (2001) states: "collective constructs, [must] be easily learned, acquired from everyday life experiences, abstract and flexible enough to be used for a wide range of situations" (p. 14). Values fulfil this task and have a number of other advantages. They are highly suitable for cross-cultural research as they are crossculturally relevant and valid and allow for both within- and between-group comparison (Hills, 2002). They are trans-situational guidelines and in this sense not tied to specific situations making it easier to assure cross-cultural equivalence for comparisons. The total number of values that people may pursue is assumed to be relatively small (Rokeach, 1973) which reduces their complexity in research. They are also less subjected to change as it is the case with attitudes (Hills, 2002). Last but not least, they may be essential in understanding the underlying motives of social norms, which are prescriptions/ proscriptions to behave in a certain way in a specific situation (Rokeach, 1973).

In this account, the Schwartz Value Survey has been referred to several times which can be understood as an indication of its popularity to measure explicit value priorities. Recent research has also been presented where the instruction of the survey has been modified to measure cultural values at the individual-level. The SVS is an excellent instrument to measure values and can be easily modified in its response scale to capture the different value types as proposed in the value taxonomy. Thus, the SVS provides a sound foundation for exploring and extending value research to enrich our understanding of values in different cultures. 


\title{
The Structure of Human Values at the Culture-level:
}

\section{A Meta-analytical Replication of Schwartz' Value Orientations Using the Rokeach Value Survey}

\begin{abstract}
Introduction
Values are considered to be one of the defining concepts for differentiating cultural groups from one another (Smith \& Schwartz, 1997). An important question is how values are structured across different societies, so that cultures can be meaningfully described and compared to each other. Schwartz (1994b, 2006) developed a value structure theory at the culture-level, which was validated with numerous samples from all inhabited continents. Applying multidimensional scaling techniques on 45 values, he was able to show that they form seven value types organized in a quasi-circumplex way in a two-dimensional space. This value configuration forms a coherent and integrated system of societal value preferences. The culture-level value structure has been shown to relate empirically to a number of nation-level indicators, such as socio-economic development, gender equality, justice perceptions and optimism (Fischer \& Chalmers, 2008; Fischer, et al., 2007; Schwartz, 2006b). Yet, before an empirical result can serve as established evidence, it must withstand the test of replicability (Amir \& Sharon, 1990).
\end{abstract}

Note. An earlier version of this chapter has been accepted for publication and is hereafter referenced as follows: Vauclair, C.-M., Hanke, K., Fischer, R. \& Fontaine, J. R. (in press). The structure of human values at the culture-level: A meta-analytical replication of Schwartz' value orientations using the Rokeach Value Survey. Journal of Cross-cultural Psychology. 
To the best of my knowledge, the culture-level structure has not been independently replicated yet $^{3}$. Hence, the first step in scrutinizing the validity of Schwartz' theory is here to test the replicability of his cultural value types. To accomplish that, I chose to conduct a meta-analysis on the Rokeach Value Survey (RVS, Rokeach, 1968, 1973). The RVS contains an additional set of value items that have either not been included in Schwartz' survey to assess values (SVS) or that have been excluded from the culturelevel analysis. I examined whether these omitted values form a value type of their own. Despite the commonly shared opinion about replication studies as not producing anything new (Neuliep \& Crandall, 1993), I will show that my replication contributes to the generation of new knowledge and prepares the ground for new theoretical perspectives.

For the sake of a more comprehensive structure of the paper, I have separated the analyses into two studies according to the aims of my research: Study 1a deals with the replicability of Schwartz' culture value structure; Study $1 \mathrm{~b}$ focuses on those Rokeach values that were omitted in Schwartz' analysis. Before presenting the results, I provide the rationale for using a meta-analysis to replicate Schwartz' culture-level value structure.

\section{Different Replication Strategies}

Aiming to replicate Schwartz' culture-level findings, I was confronted with choosing between two possible procedures: the test of reproducibility and the test of generalizability (Amir \& Sharon, 1990). These different kinds of replication strategies differ on their conceptual level, the method employed to carry them out, and also the value of their outcome. Testing the reproducibility has the aim to ensure that the

\footnotetext{
${ }^{3}$ An exception is Schwartz' (2006) culture-level analysis using the PVQ which replicated the structure of values. Yet, it is based on data from the European Social Survey project comprising European countries and Israel only.
} 
empirical results are reliable. It is important herein to conduct a study that is as identical as possible to the original one in terms of the specific context where it has been carried out, i.e. the population, the setting, and the time frame. If this so-called 'fairly precise' replication is successful, it supports the theory; if it is unsuccessful, it has the potential to impair the established theory (Rosenthal, 1991). Compared with reproducibility, replication studies concerned with the generalizability of an empirical result are located more on the opposite pole of precision and value of outcome. In the words of Rosenthal (1991), "The more imprecise the replications, the greater the benefit to the external validity of the tested relationship if the results support the relationship" (p. 5, italics added). Hence, the test of generalizability is achieved when certain aspects of the original study are modified, so that its external validity can be examined and the findings can be generalized across a larger sample or context.

\section{Testing the Generalizability of Schwartz' Theory}

In cross-cultural psychology, the actual interest for individual-level studies lies in conducting fairly imprecise replication studies. This is usually done by sampling respondents from other cultures, so that an extension of the theory in question to these cultures can be achieved if the replication is successful (van de Vijver \& Leung, 1997). If the replication fails, the theory or instrument may be specific to certain cultural groups. The same rationale can be applied to culture-level studies. If Schwartz culturelevel findings cannot be replicated with a different set of cultures, his value types may only be valid for a specific composition of cultures.

Yet, testing the generalizability of culture-level studies, such as Schwartz' cultural value dimensions, is more difficult to achieve than for individual-level studies. Culture-level studies require data collection from a wide range of different cultures, which is very costly, effort- and time-intensive. This difficulty applies also to the 
replication of them, if it involves primary data collection. A solution to overcome the problem of data collection is to carry out a meta-analysis on studies that have already been conducted in different countries (cf. Norenzayan \& Heine, 2005).

\section{Meta-analysis}

Meta-analysis can be used as a tool to obtain a cross-cultural dataset. Metaanalysis is a set of techniques that statistically combines the results of several studies to provide an answer to the overall research question (Lipsey \& Wilson, 2001). There is a distinction between a domain-based and instrument-based meta-analysis (van Hemert, 2003). The former uses a thematic domain from which cross-cultural studies are sampled. In an instrument-based meta-analysis, studies are sampled that uses the same psychological instrument. I chose to conduct an instrument-based meta-analysis since it fits my purpose to obtain a cross-cultural dataset on personal values. Yet, in terms of the specific instrument, I was confronted with two possible options: a) a meta-analysis on studies that used the SVS; or b) a meta-analysis on another value instrument containing conceptually similar values. One such an instrument is the Rokeach Value Survey (RVS), developed and published in the late 1960's by Rokeach $(1968,1973)$. The RVS is especially suitable as it has been widely used around the world (Rohan, 2000). Hence, a meta-analysis on the RVS should yield an extensive cross-cultural dataset.

There are both advantages and disadvantages regarding the two approaches mentioned above. A meta-analysis on the SVS would yield results that are better comparable to Schwartz' primary analysis, but at the same time it would comprise studies published in a much narrower time frame compared to a meta-analysis on the earlier published RVS. This may also result in the inclusion of samples that are very similar to the ones in Schwartz' analysis as most of the published papers emerge from Western countries (Quinones-Vidal, Lopez-Garcia, Penaranda-Ortega, \& Tortosa-Gil, 
2004), consisting mainly of university students as the most accessible group for empirical analyses (Norenzayan \& Heine, 2005). Eventually, this would come close to a 'fairly precise' replication which does not allow drawing conclusions on the generalizability of Schwartz' findings. In contrast, a meta-analysis on the RVS, which has been used in research for approximately 40 years, has the potential to yield a much bigger pool of studies that may allow generalizing beyond the sample characteristics of Schwartz' study.

Another point of consideration is that by choosing the RVS, there is the unique opportunity to test whether Schwartz' cultural value dimensions are also identifiable if the measurement of values is based on a different response format. The main difference between the SVS and the RVS is that the latter is based on a rank order procedure, whereas the former follows a rating instruction on a 9- point Likert scale ranging from "opposed to my values" (-1) through "important" (3) to "very important" (6) and "of supreme importance" (7). Comparing different measurement techniques that assess the same theoretical construct with the purpose of identifying the same underlying structure can be seen as another attempt of generalizing. If I find similar results with a different instrument, I can conclude that I have replicated a very robust phenomenon despite the variations in value assessment (cf. Kline, 2004).

I decided to conduct a meta-analysis on the RVS to obtain a cross-cultural dataset that would enable me testing the structure of values at the aggregated culturelevel. This implies (1) coding of the rank orders or ratings of Rokeach Values that are provided for specific samples in the studies, (2) aggregating the sample scores within cultural groups by using the arithmetic mean. This kind of meta-analysis in which the mean is coded as the variable of interest is less often reported in the literature. In conventional meta-analysis, the results are usually combined by taking effect sizes as an indicator of the strength of the relationship between two variables. Yet, meta-analyses 
using central distribution information are possible (Lipsey \& Wilson, 2001) and provide useful information for cross-cultural analyses (e.g., Dekker \& Fischer, 2008; Fischer \& Chalmers, 2008; Fischer \& Mansell, in press).

\section{Study 1a: Replicating the Culture-level Value Structure}

I conducted a meta-analysis on the Rokeach Value Survey (RVS) by coding samples' mean rankings or ratings of values as reported in the respective studies. The RVS consists of 18 terminal values (goals in life) and 18 instrumental values (modes of conduct) which are rank ordered separately by assigning low rank orders (minimum 1) to important values and high rank orders (maximum 18) to unimportant ones ${ }^{4}$. Each value is followed by a defining phrase which clarifies its meaning (see Table 3.1).

Following Schwartz' choice of method, I used multidimensional scaling (MDS) on all 36 Rokeach values to identify the culture-level value types. As the MDS is applied on a smaller set of values compared to Schwartz' (1994b) culture-level analysis (45 values), I expected that some neighbouring value types may merge. The Rokeach values may not provide as much detail in value type specification as the larger list of values in Schwartz' instrument. I did not expect to identify a Hierarchy value type as the RVS does not contain any values of this kind (e.g. social power and authority).

\section{Method}

\section{Meta-analysis}

Literature Search and Inclusion Criteria. I searched for relevant studies in the database PsycINFO covering the period 1968 (year of first publication of the RVS) to March 2006. The keywords used were Rokeach and values which resulted in 558 references.

\footnotetext{
${ }^{4}$ To facilitate the interpretation of correlations that I report later in the manuscript, I recoded the ranks so that higher numbers correspond to higher importance of the respective values and vice versa.
} 
Table 3.1: Comparison of Rokeach and Schwartz' values.

\begin{tabular}{|c|c|c|}
\hline & RVS (Rokeach, 1973) & SVS (Schwartz, 1992, 1994b) \\
\hline 1 & A Comfortable Life (a prosperous life) & - \\
\hline 2 & $\begin{array}{l}\text { A Sense of Accomplishment (lasting } \\
\text { contribution) }\end{array}$ & - \\
\hline 3 & A World at Peace (free of war and conflict) & A World at Peace (free of war and conflict) \\
\hline 4 & A World of Beauty (beauty of nature and arts) & A World of Beauty (beauty of nature and arts) \\
\hline 5 & Ambitious (hardworking, aspiring) & Ambitious (hardworking, aspiring) \\
\hline 6 & An Exciting Life (a stimulating, active life) & An Exciting Life (stimulating experiences) \\
\hline 7 & Broadminded (open-minded) & $\begin{array}{l}\text { Broad-minded (tolerant of different ideas and } \\
\text { beliefs) }\end{array}$ \\
\hline 8 & Capable (competent, effective) & Capable (competent, effective, efficient) \\
\hline 9 & Cheerful (lighthearted, joyful) & - \\
\hline 10 & Clean (neat, tidy) & Clean (neat, tidy) \\
\hline 11 & Courageous (standing up for your beliefs) & 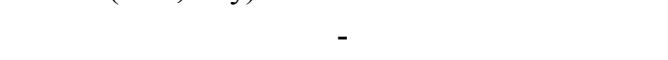 \\
\hline 12 & Equality (brotherhood, equal opportunity for all) & Equality (equal opportunity for all) \\
\hline 13 & Family Security (taking care of loved ones) & Family Security (safety for loved ones) \\
\hline 14 & Forgiving (willing to pardon Others) & Forgiving (willing to pardon Others) \\
\hline 15 & Freedom (independence, free choice) & Freedom (freedom of action and thought) \\
\hline 16 & Happiness (contentedness) & - \\
\hline 17 & Helpful (Working for the welfare of Others) & Helpful (Working for the welfare of Others) \\
\hline 18 & Honest (sincere, truthful) & Honest (genuine, sincere) \\
\hline 19 & Imaginative (daring, creative) & Creativity (uniqueness, imagination) \\
\hline 20 & Independent (self-reliant, self-sufficient) & Independent ( self-reliant, self-sufficient) \\
\hline 21 & Inner Harmony (freedom from inner conflicts) & Inner Harmony (at peace with myself) \\
\hline 22 & Intellectual (intelligent, reflective) & - \\
\hline 23 & Logical (consistent, rational) & Intelligent (Logical thinking) \\
\hline 24 & Loving (affectionate, tender) & - \\
\hline 25 & Mature Love (sexual and spiritual intimacy) & $\begin{array}{l}\text { Mature Love (deep emotional and spiritual } \\
\text { intimacy) }\end{array}$ \\
\hline 26 & National Security (protection from attack) & $\begin{array}{l}\text { National Security (protection of my nation } \\
\text { from enemies) }\end{array}$ \\
\hline 27 & Obedient (dutiful, respectful) & Obedient (dutiful, meeting obligations) \\
\hline 28 & Pleasure (an enjoyable, leisurely life) & Pleasure (gratification of desires) \\
\hline 29 & Polite (courteous, well-mannered) & Politeness (courtesy, good manners) \\
\hline 30 & Responsible (dependable, reliable) & Responsible (dependable, reliable) \\
\hline 31 & Salvation (saved, eternal life) & - \\
\hline 32 & Self-controlled (restrained, self-disciplined) & $\begin{array}{l}\text { Self-discipline (self-restraint, resistance of } \\
\text { temptation) }\end{array}$ \\
\hline 33 & Self-respect (self-esteem) & Self-respect (belief in one's own worth) \\
\hline 34 & Social Recognition (respect, admiration) & $\begin{array}{l}\text { Social Recognition (respect, approval by } \\
\text { Others) }\end{array}$ \\
\hline 35 & True Friendship (close companionship) & True Friendship (close, supportive friends) \\
\hline 36 & Wisdom (a mature understanding of life) & Wisdom (a mature understanding of life) \\
\hline
\end{tabular}

Note. Values in italics are those that were omitted in Schwartz' culture-level analysis . 
As my aim was to obtain value priorities of 'normal' adult samples belonging to different cultures, studies with children and young adolescents (younger than 16 years of age), clinical as well as delinquent samples were not included in the study. Furthermore, only those studies were selected where the original 18 terminal and 18 instrumental values had not been replaced by other values (as, for example, in Form $\mathrm{G}$ of the RVS in which happiness is replaced by health and cheerful by loyal). Furthermore, the mean or median rank orders needed to be reported for at least all 18 terminal or all 18 instrumental values. Some researchers also added specific values to the original list of Rokeach values. I dropped these additional values from the analysis and recalculated the rank orders for the 18 original values. Foreign-language articles (22 out of 130) were translated by me or bilingual speakers. The final dataset consisted of 344 samples for which value priorities were coded. These samples had been assessed in 173 independent studies. The findings had been published in $130 \operatorname{articles}^{5}$.

Samples. A summary of the sample characteristics can be seen in Table 3.2. The year of data collection of the studies ranged from 1967 to 2002. The majority of studies included in the meta-analysis were conducted in the USA, which is reflected in the large number of samples (98). The aggregated median sample size of cultural groups consisted of 349 respondents. Gender was not reported for $48 \%$ of all samples. Of those that provided this information, $47.2 \%$ of the samples were male. The age of respondents ranged from 16 to 75 . Samples were coded as falling either into the category of university students (54\% of all samples), working adults $(27 \%)$, general population $(8 \%)$ or 'others' $(11 \%)$ (e.g. seniors). In total, the meta-analysis is based on the responses of 44,047 respondents from 32 different countries and five ethnic minority groups such as Hispanics in the USA and Chinese in New Zealand.

\footnotetext{
${ }^{5}$ See Appendix A for the complete list of articles included in the meta-analysis.
} 
Similarly to Schwartz' dataset, the samples come from every inhabited continent. To compare the distribution of countries to Schwartz' initial dataset from which he derived his culture-level structure (cf. Schwartz, 1994aa, pp. 29-29), I assigned each country to geographical regions. In Schwartz' dataset, the majority of countries belonged to Europe/ Eurasia (52.3\%), followed by Asia (22.7\%) and Latin America (9.1\%). Countries from Oceania, North America and Sub Sahara constituted each $4.5 \%$ of the sampled countries and the Middle East was represented by $2.3 \%$ of all countries. My dataset consisted of proportionally less countries from Europe/ Eurasia (27\%) and the Sub Saharan (2.7\%) region and more countries from Asia (29.7\%), the Middle East (16.2\%), and Oceania (8.1\%). The proportions of countries representing Latin America (10.8\%) and North America (5.4\%) were about the same. In total, $62.2 \%$ of the countries overlapped between Schwartz' and my study.

Computation and Analysis. For each sample, the reported mean or median rank orders were coded. Mean ratings were coded in cases where a rating procedure $(10.5 \%$ of all samples) instead of the original ranking was used. To make this coded information maximally comparable, I rank ordered terminal and instrumental values again within each sample. I rank ordered the two sets of values separately. Before aggregating samples at the culture-level, I weighted all coded rank orders by their sample size in order to give more weight to large sample sizes. However, the weighting procedure transforms ordinal data into interval data. To obtain again ordinal data at the aggregated culture-level, I rank ordered the values, this time within each cultural group. For the nation Slovakia, only the instrumental values were available and for Slovenia only the terminal values. These countries were excluded from the analysis in Study 1a, as the statistical procedure I was employing deletes missing values listwise. Thus, all following analyses in this section are based on 30 nations and five ethnic minority groups. 
Table 3.2: Sample characteristics of the meta-analysis per country.

\begin{tabular}{|c|c|c|c|c|}
\hline & & k & $\mathrm{N}$ & \\
\hline Country & $\begin{array}{l}\text { Year of data } \\
\text { collection }\end{array}$ & samples & participants & Respondents \\
\hline Australia & $1986-2002$ & 64 & 5,589 & $\begin{array}{c}\text { University students, } \\
\text { Working population, } \\
\text { Others }\end{array}$ \\
\hline Bangladesh & 1982-2002 & 2 & 200 & University students \\
\hline Brazil & 1969-1988 & 18 & 3,136 & $\begin{array}{l}\text { General population, } \\
\text { University students }\end{array}$ \\
\hline Canada & 1974-1990 & 7 & 382 & $\begin{array}{c}\text { University students, } \\
\text { Working population, } \\
\text { Others }\end{array}$ \\
\hline Chile & 1985 & 1 & 70 & University students \\
\hline China & 1985-1997 & 14 & 2,424 & University students \\
\hline $\begin{array}{l}\text { China, Hong Kong } \\
\text { SAR }\end{array}$ & 1988-2002 & 5 & 1,672 & $\begin{array}{l}\text { University students, } \\
\text { Others }\end{array}$ \\
\hline Egypt & 1993 & 1 & 658 & Working population \\
\hline Finland & 1981 & 4 & 172 & University students \\
\hline Germany (West) & 1974-1990 & 5 & 1,093 & $\begin{array}{l}\text { University students, Working } \\
\text { population }\end{array}$ \\
\hline Israel & 1966-1985 & 25 & 1,059 & University students \\
\hline Japan & 1978-2002 & 9 & 709 & $\begin{array}{l}\text { University students, Working } \\
\text { population }\end{array}$ \\
\hline Jordan & 1986 & 2 & 2,000 & University students \\
\hline Malaysia & 1982-2002 & 2 & 150 & University students \\
\hline Mexico & 1983 & 6 & 168 & Working population \\
\hline New Zealand & 1974-2002 & 4 & 240 & University students \\
\hline $\begin{array}{l}\text { Papua New } \\
\text { Guinea }\end{array}$ & 1971-2002 & 8 & 1,645 & $\begin{array}{l}\text { University students, } \\
\text { Others }\end{array}$ \\
\hline Poland & 1982-1998 & 10 & 1,276 & $\begin{array}{c}\text { University students, } \\
\text { Others }\end{array}$ \\
\hline Romania & 1997 & 2 & 200 & $\begin{array}{c}\text { University students, } \\
\text { Others }\end{array}$ \\
\hline
\end{tabular}




\begin{tabular}{|c|c|c|c|c|}
\hline & & k & $N$ & \\
\hline Country & $\begin{array}{l}\text { Year of data } \\
\text { collection }\end{array}$ & samples & participants & Respondents \\
\hline Singapore & 1988 & 1 & 65 & University students \\
\hline Slovakia & 1995 & 2 & 179 & Others \\
\hline Slovenia & 1985 & 2 & 1,409 & Working population \\
\hline South Africa & 1984 & 3 & 123 & Working population \\
\hline Spain & $1982-2001$ & 2 & 632 & $\begin{array}{l}\text { University students, } \\
\text { Others }\end{array}$ \\
\hline Sweden & 1980 & 2 & 25 & Others \\
\hline Taiwan & 1982-2001 & 2 & 200 & University students \\
\hline Turkey & 1980 & 7 & 1,610 & $\begin{array}{l}\text { University students, } \\
\text { Working population }\end{array}$ \\
\hline Ukraine & 1969 & 2 & 84 & $\begin{array}{l}\text { University students, } \\
\text { Working population }\end{array}$ \\
\hline United Kingdom & $1975-1988$ & 14 & 917 & $\begin{array}{c}\text { University students, } \\
\text { Working population, } \\
\text { Others }\end{array}$ \\
\hline $\begin{array}{l}\text { United States of } \\
\text { America }\end{array}$ & 1967-2001 & 98 & 14,710 & $\begin{array}{c}\text { General population, } \\
\text { University students, } \\
\text { Working population, } \\
\text { Others }\end{array}$ \\
\hline Vietnam & 1977 & 1 & 349 & General population \\
\hline Malaysia Chinese & 1982 & 1 & 79 & University students \\
\hline NZ Chinese & 1982 & 1 & 100 & University students \\
\hline Hispanics in USA & 1987-1993 & 2 & 161 & $\begin{array}{l}\text { General population, } \\
\text { University students }\end{array}$ \\
\hline Africans in Egypt & 1992 & 1 & 43 & Working population \\
\hline $\begin{array}{l}\text { Non-Egyptian Arabs } \\
\text { in Egypt }\end{array}$ & 1992 & 1 & 101 & Working population \\
\hline Totals & 1967-2002 & 344 & 44,047 & \\
\hline
\end{tabular}

Note. The year of data collection is based on the information given in the respective papers. If this information was missing, I calculated an estimated year of data collection by subtracting the average delay between data collection and publication ( 3.44 years) as inferable from some papers. 
I applied a non-metric multidimensional scaling procedure (MDS; PROXSCAL in SPSS 14.01) on the Rokeach value rankings at the aggregated culture-level. MDS is like factor analysis a data reduction method, but with the difference that it visualizes the data structure. MDS represents the value items as points in a geometrical space in such a way that the distances between the points reflect the associations between the items, with small distances corresponding to strong positive associations and large distances to zero or negative associations (Borg \& Groenen, 1997). The dissimilarity measure for the MDS was based on the Spearman correlations between all 36 value items.

The Rokeach-MDS also includes values for which Schwartz' (1992, 1994b) has reported unstable meaning across cultures or for which cross-cultural equivalence has not been established yet (see Table 3.1). There are two reasons why I decided to use all Rokeach values to derive a MDS configuration: (1) Recent analysis suggested that shifts for values that were found to be unstable in meaning are mostly due to other reasons than cross-cultural inequivalence (see below in more detail, Fontaine, Poortinga, Delbeke, \& Schwartz, 2008); (2) if there was indeed a problem of unstable meaning of values, it would increase random error at the culture-level (Fontaine, 2008), and thus lead to very low correlations of those items with other items. Empirically, this would most likely be shown by value items that take a central position in the MDS, since the correlations - and by implication the distances - would be low and about equal to all other values. Therefore, I would be able to detect unstable meaning of values in the MDS and I could report for which values this is the case. I concluded that it is important to include all values, otherwise important content might be omitted.

I imposed a two-dimensional solution on the value items. The Kruskal stress index (Kruskal \& Wish, 1978) indicates how well the distances in the MDS solution approximate the original distances. A lower Kruskal stress (with a minimum of 0 ) suggests better solutions. The Kruskal stress for the two-dimensional configuration was 
relatively high with a value of .25 . While adding dimensions can decrease the stress to an acceptable fit value, there is a trade-off between improving fit and reducing the interpretability of the solution. According to Kruskal and Wish (1978), the fit index should be used flexibly when a MDS configuration is seen as the foundation on which to display clusters of items: a two-dimensional configuration can be more useful than a three- or four-dimensional one. To the best of my knowledge, Schwartz provides no stress indices for his culture-level analysis. Considering that I aimed to replicate Schwartz' model, I decided to keep the two-dimensional solution for the RVS-MDS.

\section{Comparison with the SVS}

I used archival datasets of the Schwartz Value Survey (SVS) collected by Schwartz and his colleagues (Schwartz, 1992, 2005) between 1988 and 2002, including samples of both students and teachers. Teacher data was available from 55 nations $(\mathrm{N}=$ $15,975)$ and student data from 66 nations $(\mathrm{N}=26,024)$. Further information on the samples is available in Schwartz (2005). I analyzed the SVS value structure separately for the student and teacher samples. The country level structure was derived from ztransformed Euclidean distances based on the aggregated country means of students' and teachers' responses. Using PROXSCAL in SPSS 14.01, an interval-level MDS was run.

I applied the MDS to all 55 value items ${ }^{6}$. Schwartz only analysed those 45 values that did not shift too much from the hypothesized value structure at the individual-level. Large value shifts across cultural groups indicate a shift in meaning and therefore a lack of cross-cultural equivalence. Schwartz (1992, 1994b) used the configurational verification approach to detect these value shifts. Yet, very recent

\footnotetext{
${ }^{6}$ The SVS consists of 58 values. I limited the analysis to 55 value items as the values 'detachment', 'privacy', and 'self-indulgent' were not consistently included in all studies.
} 
individual-level analyses using more rigorous methods showed that the average value structure across cross-cultural samples based on all 55 values is very robust (Fontaine, et al., 2008). Furthermore, Fontaine (1999) found that the actual size of shifts for excluded values from the culture-level analysis (e.g. mature love) were not systematically larger than for included values (e.g. clean, ambitious). Therefore, I used all 55 value items to derive the culture-level value structure.

Qualitative Comparison. Schwartz (1994b) hypothesized a quasi-circumplex model consisting of seven axes that form wedge-like regions emerging from a common origin (see Figure 1.2). He employed the configurational verification approach meaning that he checked visually whether the empirical structure was similar to the theoretically postulated one. This was done by drawing boundary lines around clusters of value items, while avoiding overlap between regions as much as possible (see Schwartz, 1992, p. 22 for details). Then, partition lines between these boundaries were drawn. I used the same approach on the Rokeach values. First, I examined which of the Rokeach values are conceptually similar to Schwartz' values. Schwartz (1992) indicated himself that the SVS contains some values which are "identical" (Schwartz, 1992, p. 17) to the RVS. I compared both surveys and found a conceptual overlap of 28 values altogether (see Table 3.1). Twenty-three of these values were part of Schwartz' culture-level value structure. Therefore, I used these 23 matching values as a guideline to employ the configurational verification approach.

Quantitative Comparison. I compared Schwartz' and the RVS-MDS configuration more precisely by correlating the coordinates of the 23 matching values to get an objective measure of their similarity. As can be seen in Table 3.1, I have identified another five Rokeach values that are conceptually similar to Schwartz' values, but were excluded from his culture-level analysis. Thus, additionally to the 23 matching values, I also examined the degree of similarity between all 28 matching values. 
Prior to the correlation of value coordinates, the Rokeach and Schwartz' MDS needed to be transformed to maximal similarity. The reason is that MDS can produce configurations that are altered through translation, reflection, rotation or shrinking and dilatation (Borg \& Groenen, 1997). If the RVS and SVS configurations are not transformed before they are correlated, their similarity may be underestimated. I used Generalized Procrustes Analysis (GPA, Commandeur, 1991) to transform the data. I performed two separate GPA's: one on 23 and another one on 28 matching values. Since I analyzed Schwartz' student and teacher data separately, there were three different MDS configurations to be transformed with GPA: (1) the configuration for the Rokeach values, (2) the configuration for Schwartz' students' values, and (3) the configuration for Schwartz' teacher's values.

\section{Results and Discussion}

\section{Configurational Verification Approach}

The General Value Structure. Figure 3.1 shows the MDS on all 36 Rokeach values in a two-dimensional space. The configurational verification approach revealed that there were four value clusters organized in a circular order that match Schwartz' value clusters. As can be seen in the figure, the adjacent and thus compatible value orientations Egalitarianism and Harmony merged into a single value cluster. Similarly, Affective and Intellectual Autonomy merged together ${ }^{7}$. In line with Schwartz' predictions, Embeddedness is opposite to Autonomy values and Egalitarianism and

\footnotetext{
${ }^{7}$ It may be possible that the merging of value types is caused by the absence of hierarchy values. To confirm that, I conducted a follow-up analysis on Schwartz' data. I applied a MDS on all 45 values that Schwartz used for the culture-level analysis, but I excluded the hierarchy values. The MDS solution shows that the overall value structure as such does not change. Yet, the mastery value type is more stretched and egalitarianism and harmony as well as intellectual and affective eutonomy are not clearly separable anymore. Drawing partition lines would result in at least one value that is misplaced in each of the value dimensions. This suggests that the distinction between these value types may indeed to some extent be driven by their differing relations with hierarchy values.
} 
Harmony are opposed to Mastery. This confirms the organization of culture-level value types according to their conflicts and compatibilities. In summary, it can be concluded that the overall structure of conflicting value orientations is present in the solution.

Shifting Values. There are five values, independent, capable, ambitious, a world at peace, and self-controlled (underlined in Figure 3.1), that are located in different value clusters as compared to Schwartz' solution. The arrows in Figure 3.1 indicate where they should fit in Schwartz' configuration. It is noteworthy that these values do not shift to opposite value types, but rather to adjacent ones with which they are theoretically compatible. Furthermore, the location of some of these values shows that they are meaningfully associated with the value types they shift to. The values independent and capable, for example, are not located in Mastery but in the Autonomy value domain. Independent can be regarded as a key value for both Intellectual and Affective Autonomy. Similarly, capable could be seen as a representative value for Intellectual Autonomy. Self-controlled falls into the domain Egalitarianism/ Harmony and not into Embeddedness as in Schwartz's findings. Yet, both solutions seem to be meaningful: Being self-controlled as fitting into the social group is compatible with Embeddedness, whereas being self-controlled as a moral obligation (consistent with values such as responsible, helpful etc.) implies living harmoniously with the natural and social world, which is corroborated by its location within the Harmony value cluster. Besides these possible explanations in terms of different meanings of shifting values, the value shifts may also be due to methodological reasons, including sampling of different cultural groups, a different composition of countries representing geographical regions, and differences in translation of items. It is difficult to identify the precise reasons with the present study. 


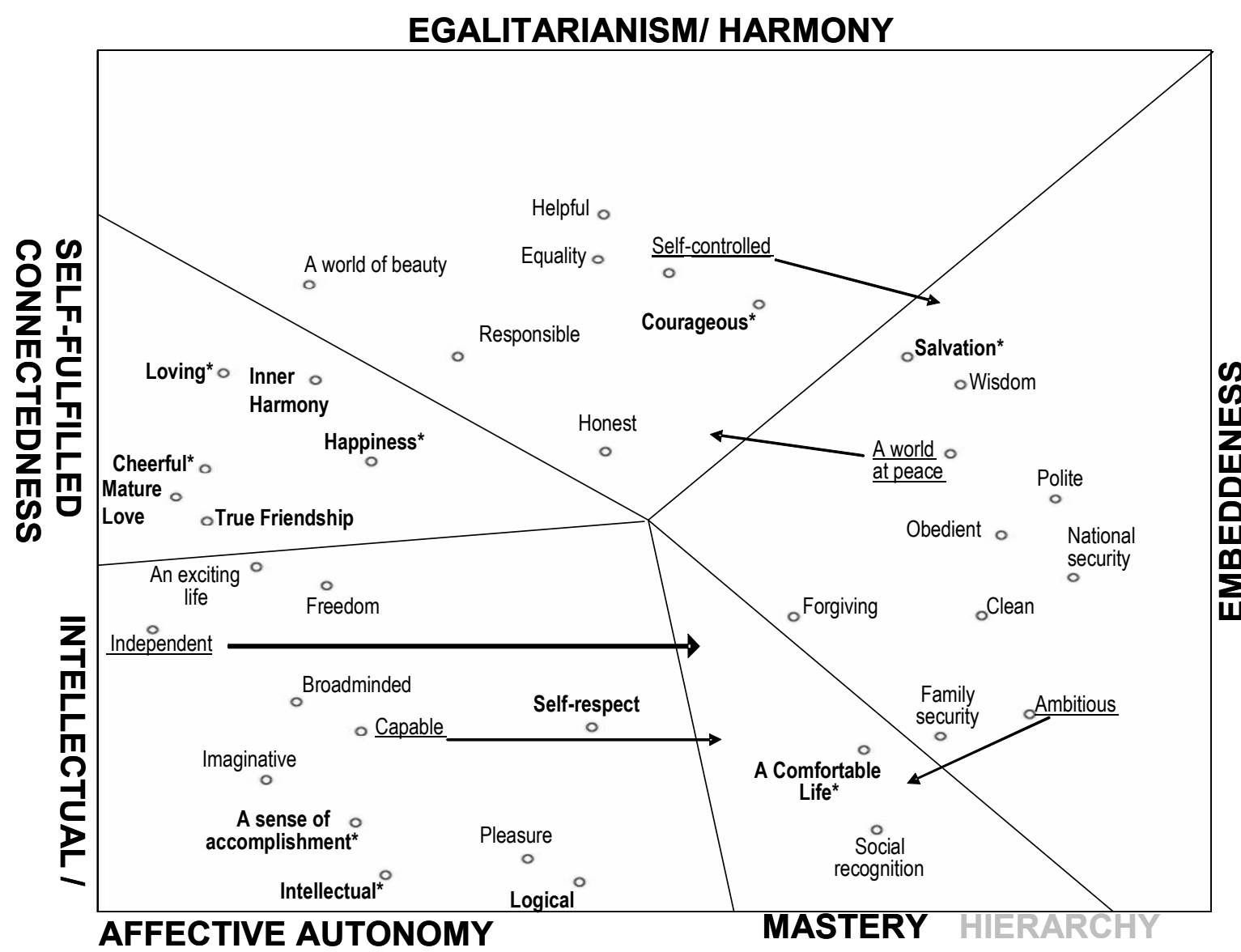

Figure 3.1: MDS representation of all 36 Rokeach values

Note. This MDS solution displays not the GPA transformed value configuration, since this can only be done for matching values and would not allow displaying all 36 Rokeach values. However, an examination of the transformed and untransformed solutions showed that they are very similar.

Values in bold were not included in Schwartz' (1994b) culture-level analysis; values with an asterisk do not appear in the SVS. Underlined values are located in different value types compared to Schwartz' solution. Arrows indicate their location in Schwartz (1994).

To summarize, I was able to replicate four distinct value clusters. Taking into account that it is not possible to replicate the Hierarchy value type as there are no Hierarchy values in the RVS, I can state that conceptually I was able to identify four out of six of Schwartz' value types. The reduced number of emerging value clusters may be a result of under-representation of value items in the respective value domains (Fontaine, 2005) and the absence of Hierarchy values. A comparison with Schwartz' MDS shows that five values shifted to adjacent value types. 


\section{Correlational Analysis}

Similarity of the Value Structure. In the next step, I moved beyond the configurational verification approach as a descriptive procedure and applied a more stringent method to measure the degree of similarity between the Rokeach and Schwartz' value structure. Table 3.3 shows the Spearman correlation coefficients for the value configuration based on 23 and 28 matching values after transforming the MDS solutions to maximal similarity with GPA. As can be seen, all correlations are significant and there is only little difference if congruence is tested for 23 matching values (upper diagonal) or 28 matching values (lower diagonal). The correlations for RVS and SVS value structures range from .48 to .85. There is a slightly higher similarity to the students' SVS-MDS than to the teachers' MDS. The first dimension shows a consistently higher correlation than the second dimension - a pattern that is also noticeable for correlations between the student and teacher value configuration of the SVS.

Table 3.3: Spearman correlations between Schwartz' and Rokeach culture-level value structure based on MDS.

\begin{tabular}{|c|c|c|c|c|c|c|}
\hline & $\begin{array}{l}\text { RVS } \\
\text { Dim } 1\end{array}$ & $\begin{array}{l}\text { RVS Dim } \\
2\end{array}$ & $\begin{array}{l}\text { SVS } \\
\text { student } \\
\text { Dim } 1\end{array}$ & $\begin{array}{l}\text { SVS student } \\
\text { Dim } 2\end{array}$ & $\begin{array}{l}\text { SVS teacher } \\
\text { Dim } 1\end{array}$ & $\begin{array}{l}\text { SVS } \\
\text { teacher } \\
\text { Dim } 2\end{array}$ \\
\hline RVS & & & & & & \\
\hline $\begin{array}{l}\text { Dim } 1 \\
\text { RVS }\end{array}$ & 1.00 & -.03 & $.85 * * *$ & -.14 & $.76 * * *$ & .07 \\
\hline $\begin{array}{l}\operatorname{Dim} 2 \\
\text { SVS student }\end{array}$ & -.04 & 1.00 & -.06 & $.51 * *$ & -.07 & $.59 * *$ \\
\hline $\begin{array}{l}\text { Dim } 1 \\
\text { SVS student }\end{array}$ & $.82 * * *$ & -.08 & 1.00 & -.02 & $.93 * * *$ & .10 \\
\hline $\begin{array}{l}\text { Dim } 2 \\
\text { SVS teacher }\end{array}$ & -.20 & $.56^{* *}$ & -.06 & 1.00 & .08 & $.64 * * *$ \\
\hline $\begin{array}{l}\text { Dim } 1 \\
\text { SVS teacher }\end{array}$ & $.75 * * *$ & -.03 & $.95 * * *$ & .02 & 1.00 & .23 \\
\hline $\operatorname{Dim} 2$ & .01 & $.48^{* *}$ & .03 & $.64 * * *$ & .10 & 1.00 \\
\hline
\end{tabular}

Note. $* p<.05, * * p<.01, * * * p<.001$.
Dim 1 and 2 refer to dimensions 1 and 2 in the two-dimensional space of the MDS. The lower diagonal shows the correlations for $\mathrm{N}=28$ matching values and the upper diagonal for $\mathrm{N}=23$ matching values. The correlations were computed after transforming the value configurations to maximal similarity with Generalized Procrustes Analysis (GPA). 
Considering that the RVS data has been collected with another instrument, over a different period of time and with different independent samples, the correlations show that the cultural value structure is quite robust. Overall, the results reflect the finding of the configurational verification approach, namely that there is a considerable degree of similarity between the SVS and RVS value structures.

Similarity of the Value Profiles of Countries. To test whether the value profile of countries obtained with the RVS data is similar to the SVS data, I also correlated country scores for the value types. The scores for value types were obtained by computing the mean importance ratings of the value items that represent them. Since Affective and Intellectual Autonomy merged into a single value type in the RVS- MDS, as well as Egalitarianism and Harmony, I could not differentiate between them. Thus, I had five Rokeach value types to correlate with the corresponding Schwartz' value types. In order to increase reliability of SVS-country scores, I combined the teacher and student samples (see also Schwartz, 2006b). For ease of comparison purposes with the RVS data which only showed a single Autonomy value type, I also combined Intellectual and Affective Autonomy for the SVS data. For Jordan, only the teacher scores were available and for Romania, South Africa and Ukraine only the student scores. I obtained 29 matching countries between the RVS and SVS dataset. I corrected for scale use by centring the responses as advised by Schwartz (1992, 2007). Since I expected that matching value types yield similar rankings of countries, I used one-tailed statistical tests. I found the strongest relationship for the Rokeach and Schwartz' Embeddedness value type $\left(r_{s}=.67, p<.001\right)$, followed by a moderate and marginally significant correlation between the RVS- and SVS-Mastery value type $\left(r_{s}=.31, p=\right.$ .06). There was no significant correlation between the SVS- and RVS-Autonomy value type $\left(r_{s}=.14, p=.23\right)$. There was also no significant correlation for RVSEgalitarianism/ Harmony and SVS-Egalitarianism $\left(r_{s}=.24, p=.11\right)$ or SVS-Harmony 
$\left(r_{s}=-.08, p=.34\right)$. Thus, for the more 'collectivistic-oriented' values, the country scores from the RVS-data and the SVS-data showed a considerable degree of similarity. Yet, for the value types on the other side of the value circle, i.e. the more 'individualisticoriented values, I found no significant relationships.

There are several possible reasons for this. It is noteworthy that the correlations were less well confirmed for those value types that merged with other value types or consisted only of a few value items. A second important issue is that the RVS data covers a long time period during which 'individualistic' values may have been shifting in their relative importance (Inglehart, 1997; Inglehart \& Baker, 2000). As a consequence the position of countries relative to the scores derived from Schwartz are likely to be different. While this can cause deviations in scores for specific countries, it does, however, not point to a real difference in the value structure as the position of values relative to each other was well replicated.

Robustness of the Rokeach Value Structure. I conducted a follow-up analysis with the Rokeach dataset to test whether the structure is replicable with sub-samples consisting of different sample types. I was to some extent restricted in the analyses since many countries were only represented by a few samples in the meta-analytical dataset which does not allow testing the direct impact of sample type on structural stability. Hence, I split the non-aggregated dataset, i.e. the sample-level dataset, into two subsamples consisting either of student or non-student samples. I again aggregated these two datasets at the culture-level. The student sample dataset contained data from students of 27 different cultural groups. The non-student dataset contained primarily data from the working or general population from 24 cultural groups. There was an overlap of 15 cultural groups between the two sub-samples. I then performed a MDS on each of the two sub-samples. Afterwards, the MDS configuration for the student and non-student sub-sample were rotated to maximal similarity with GPA. Spearman 
correlations of the two configurations for all 36 values revealed that the first dimension correlated $.72(p<.001$, one-tailed $)$ and the second dimension $.60(p<.001$, one-tailed $)$. Similar to the comparison with Schwartz' value structure, I found that the second dimension correlated somewhat less than the first dimension. Considering that the two sub-samples consisted of different sample types coming to some extent from different countries, I concluded that the Rokeach value structure was replicable. Specific sample characteristics seem to have had little impact on the overall structure.

\section{Rokeach Values Excluded in Schwartz’ Study}

The other focus of the study was aimed at the 13 Rokeach values that were not included in Schwartz' study on cultural value orientations. A visual examination of these values showed that they were not in the centre of the circle where they would be expected, if they were indeed unstable in their meaning across cultural samples. Therefore, a culture-level analysis of these values was meaningful. Schwartz did not rule out the possibility that other value types might be missing in his value circle. It may be possible that the excluded values form an additional value type of their own.

Value Items Falling into Other Value Types. Figure 3.1 shows in bold the 13 values that Schwartz omitted from his analysis, either because they were not included in the survey or because they did not pass his test of cross-cultural equivalence (the latter are additionally marked with an asterisk). Most of the values fall into value domains supporting their underlying meaning: courageous (standing up for your beliefs) clusters together with the 'moral obligation' values such as helpful and responsible. Salvation falls into the value type Embeddedness confirming its religious or conservative nature. A comfortable life is assigned to Mastery which can be seen as the final consequence of leading a self-assertive life. A sense of accomplishment, intellectual, self-respect, and 
logical fit very well into the value type Autonomy, capturing the intellectual aspect of Autonomy.

Values Forming a Separate Value Cluster. The remaining Rokeach values loving, cheerful, and happiness merit a closer examination. These three values were not included in the SVS, but clearly cluster together with the values true friendship, mature love and inner harmony that Schwartz excluded from his culture-level analysis. They fall in-between Autonomy and Egalitarianism/ Harmony. I suggest that this cluster represents a new value domain consisting of self-focused values on the one hand and other-focused values on the other hand. The self-focused values (cheerful, happiness, inner harmony) refer to a strongly positive emotional state of mind. The other-focused values represent mature relationships with profound feelings and attachment to others. I labelled this value cluster Self-fulfilled Connectedness (SFC) emphasizing the joint qualities of a sense of flourishing and satisfaction in life as well as profound attachment to others.

Study $1 \mathrm{~b}$ is devoted to examine this new value type. The aim is to answer the following questions: What is its meaning and correlates? Does this value type add something to what we already know about cultural values? Or in other words: Does the new value type have incremental validity?

\section{Study 1b: Examining the Separate Value Cluster}

Based on the meta-analytical Rokeach data, I found that $S F C$ is a value type that is located adjacent to Autonomy and opposed to Embeddedness in the value circle. This is a first indication of its underlying meaning. It suggests that $S F C$ is an individualistic value orientation, also fitting with the fact that individualism/ collectivism is a very broad theme in research on cross-cultural differences (Inglehart, 2006). Value orientations such as individualism/ collectivism (Hofstede, 1980), Autonomy/ 
Embeddedness (Schwartz, 2006), as well as survival/ self-expression (Inglehart \& Welzel, 2005), all reflect the common idea to what extent a given society emphasizes autonomous human choice (Inglehart, 2006). SFC may be another one of them. To make sure that $S F C$ is indeed closely related to 'individualistic' values of the value circle, I tested its correlation with all other value types using the independent dataset from Schwartz. Due to the circumplex structure of Schwartz' culture-level value theory, correlations of SFC and Schwartz' cultural value orientations should produce a sinusoid association, i.e. correlations should first decrease and then again increase when moving away from $S F C$ in the value circle. Thus I hypothesized that:

H1: SFC and compatible value types, i.e. Autonomy and Egalitarianism, yield significant positive correlations. The conflicting value type Embeddedness shows the strongest negative correlation.

\section{Individualism and Relatedness}

Despite the fact that different value dimensions assessing individualism/ collectivism share a great deal of commonality in their underlying meaning, they also have been found to capture unique aspects of culture (cf. Schwartz, 2006). This may be equally true for $S F C$. Individualism and collectivism are multidimensional constructs (Berry, Poortinga, Segall, \& Dasen, 2002) and SFC may measure one aspect of individualism that is not included in Schwartz' value types of Autonomy. In Schwartz' conceptualization, the focus of Autonomy is on the possibility to pursue hedonistic experiences (Affective Autonomy) and to follow own ideas and intellectual aspirations (Intellectual Autonomy). SFC appears to go beyond this 'ego-centric' focus. In SFC there is a synthesis of self- and other-focused values, i.e. a sense of personal gratification and relatedness. This may appear as a contradiction at first sight, yet, there 
is theoretical evidence that this synthesis may be a meaningful variation of an individualistic value orientation at the societal-level.

Hofstede (1980), for example, explicitly included relatedness in the form of 'concern for the immediate family' into his definition of individualism. Waterman (1981) pointed out that individualism does not necessarily mean disconnectedness or alienation from others. He argued that societies may develop an 'ethical kind of individualism', in which not only self-determined choices and the pursuit of personal goals are important, but also self-chosen and pro-social interdependencies. These kinds of societies derive a number of social benefits from emphasizing interrelatedness compared to societies emphasizing purely self-focused values. Individuals in 'interrelated' societies engage in pro-social behaviours not because they have to, but because they experience it as personally satisfying. The well-being of others becomes a function of their own personal well-being. According to Waterman (1981), social relationships that involve the most extensive mutuality and interdependence are friendship and romantic love, which are also values found in SFC. From this follows that societies emphasizing SFC should show higher levels of well-being and consequently lower levels of depression. Furthermore, pro-social behaviours in these countries should be motivated by autonomous reasons such as personal satisfaction. I predicted that:

$\mathrm{H} 2$ : SFC is positively related to well-being and inversely to depression.

H3: SFC is positively correlated with pro-social behaviour motivated by intrinsic reasons.

\section{Pursuit of Non-material Goals}

Another indication of the meaning of $S F C$ is that it contains values which are of non-material nature. This resembles the postmaterialism value orientation proposed by 
Inglehart (1997). Societies with a postmaterialist orientation emphasize self-expression and belonging besides intellectual and aesthetic satisfaction. A materialist orientation constitutes the opposite pole of this value dimension and goes along with an emphasis on survival, as expressed by values which relate to economic and physical security. Inglehart also offers an explanation why materialist values are prioritized in certain societies. He draws upon the scarcity hypothesis which says that a materialist orientation is prevalent in societies where the economic and physical security is not guaranteed. Once these basic concerns are settled, there is a shift to postmaterialist values and non-material ambitions become primary concerns. He empirically showed that advanced industrial societies shift from materialist to postmaterialist values. This phenomenon is not uniquely Western but appears in any society that has experienced the transition to high mass security. Therefore, the hypotheses for $S F C$ were:

H4: SFC is positively related to postmaterialism.

H5a: According to the scarcity hypothesis, SFC should be more strongly endorsed in countries with higher level of socio-economic security.

H5b: The positive relationship between SFC and socio-economic security is not unique to Western societies.

\section{Method}

Measures

Values. I averaged the country-level mean scores for both the terminal values mature love, true friendship, happiness, and inner harmony as well as the instrumental values loving and cheerful as obtained from the meta-analysis. In that way, I derived a single country score for the new value type $S F C$ (see Table 3.4). For the nation Slovakia, only the instrumental values were available and used for the computation of 
the country score. Similarly, I used only the terminal values to calculate the country score for Slovenia as data were only available for terminal values ${ }^{8}$.

I computed country scores for Schwartz' cultural value types with the same archival dataset I used in Study 1a. In accordance with Schwartz' (1994b) procedure, I used the same 45 values with which Schwartz obtained the seven value types Egalitarianism, Harmony, Embeddedness, Hierarchy, Mastery, Affective Autonomy, and Intellectual Autonomy. To enhance the reliability of the values, I combined student and teacher data. Prior to correlational analyses, I corrected for scale use by centring the responses as advised by Schwartz (1992, 2007a).

Subjective Well-being. I obtained country scores on Subjective Well-being (SWB) from Diener, Diener and Diener (1995). They used data of national SWB surveys compiled by Veenhoven (1993). SWB comprises questions about people's happiness and their life satisfaction and is therefore a relatively comprehensive assessment of people's state of well-being.

Depression. Country indices of depression were derived from van Hemert, van de Vijver and Poortinga (2002). The authors carried out a cross-national meta-analysis on studies using the Beck Depression Inventory (BDI) in normal populations and gathered data from 28 countries. Aggregated and standardized BDI mean scores at country-level (weighted by sample size) were directly obtained from van Hemert.

Post-materialism and motives for helping behaviour. I used data on the materialism/ post-materialism value orientation from all waves (1981-2004) of the European and World Value Survey study which can be downloaded from the World Value Survey webpage 9 . Data were available from individuals belonging to 78 countries

\footnotetext{
${ }^{8}$ Note that in Study 1a, Slovakia and Slovenia were excluded from the MDS due to listwise deletion of missing values. In Study 1b, I ran all correlational analyses with and without the two countries and found very similar results. To enhance the sample size and therewith the reliability of results, I decided to report the analyses that includes these two countries.

${ }^{9}$ Retrieved, October 2008, from http://www.worldvaluessurvey.org/
} 
whose responses were aggregated at the country-level. I used the twelve-item instead of the four-item scale as it has been found to be more reliable (Inglehart, 1990). It consists of six questions measuring materialism (i.e. attitudes towards physical security and economic security) and six items measuring postmaterialism (i.e. non-material goals).

I chose two items from the same database which assess autonomous motives for engaging in voluntary work (i.e. 'personal satisfaction' and 'gaining new skills and useful experiences'). I also chose two items tapping to extrinsic reasons (i.e. 'did not want to, but could not refuse' and 'to make a contribution to my local community') to examine the discriminant validity of $S F C$. Data were available from the year 1990, from individuals belonging to 35 countries, which were again aggregated at the country-level.

Basic Need Fulfillment. I obtained several country indices related to the socioeconomic development of nations. When possible, I computed average scores of these indices for the last 40 decades to deal with the fact that the Rokeach data had been collected across different times (see also Table 3.2). I took the Human Development Index (HDI) which combines indicators of life expectancy, educational attainment and income. It is a single statistic which serves as a reference for both social and economic development of a country. The data were available from 1975 to 2005 and published in the Human Development Report of the United Nations Development Programme (UNDP, 2006). I obtained the Gross Domestic Product(GDP) per capita from 1968 to 2003 as a measure of income (Maddison, n.d.). I derived measures of individual rights through the Gender Empowerment Measure (GEM). The GEM is published in the Human Development Report 2007/ 2008 (UNDP, 2006) and indicates the current national level of equal opportunities for men and women concerning political and economic participation, decision power and command over resources. I also included indices measuring the level of political rights (e.g. freedom of the press and freedom to peacefully assemble) and of civil liberties (e.g. freedom of religion and the right to fair 
trial) as assessed by Freedom House (n.d.). These civil rights indices were available for the years 1972 to 2005 .

Since these five indices were highly correlated, I performed a principal component analysis. The scree plot indicated a one-factor solution (eigenvalues: 4.07, $0.64,0.21,0.07,0.02)$ accounting for $81.32 \%$ of the total variance. I labelled it "Basic Need Fulfillment Index" (BNFI) and obtained a single BNF Index for each country by standardizing each of the five indices and then averaging the standardized scores. Countries that score high on BNFI show higher level of human development, higher gross domestic product per capita, more equal opportunities between men and women, and more political rights and civil liberties. Countries with the highest and lowest score on this index were the United States and Egypt respectively. An overview of all country scores that were used in the analyses are presented in Table 3.4.

\section{Test of Incremental Validity}

I have so far interpreted $S F C$ as an individualistic value orientation, sharing some similarity with Autonomy. Since SFC is conceptually very close to Autonomy, I examined whether $S F C$ significantly explains unique variance in the dependent variables over and above Autonomy. Thus, I ran six sets of regression analyses, i.e. one for each dependent variable. For each regression analysis Schwartz' data on Autonomy was entered as a predictor in the first block and $S F C$ was entered as a predictor in the second block. To reduce the likelihood of multicollinearity, I combined Schwartz' Intellectual and Affective Autonomy into one single Autonomy value type. 
Table 3.4: Relative ranking of SFC and socio-economic indicators of 32 countries.

\begin{tabular}{|c|c|c|c|c|c|c|c|c|c|}
\hline \multirow[b]{2}{*}{ Nation } & \multirow[b]{2}{*}{$\begin{array}{c}\text { Mean } \\
\text { ranking } \\
\text { score of SFC }\end{array}$} & \multirow[b]{2}{*}{$\begin{array}{l}\text { Subjective } \\
\text { Well-being }\end{array}$} & \multirow[b]{2}{*}{ Depression } & \multirow[b]{2}{*}{ Postmaterialism } & \multirow[b]{2}{*}{ BNFI\$ } & \multicolumn{4}{|c|}{ Motives for voluntary work } \\
\hline & & & & & & $\begin{array}{c}\text { 'Personal } \\
\text { satisfaction' }\end{array}$ & $\begin{array}{c}\text { 'Good } \\
\text { experience' }\end{array}$ & $\begin{array}{l}\text { 'Contribution to } \\
\text { community' }\end{array}$ & $\begin{array}{c}\text { 'Could } \\
\text { not } \\
\text { refuse' }\end{array}$ \\
\hline Australia & 14.33 & 1.02 & .05 & 2.75 & 1.14 & - & - & - & - \\
\hline Bangladesh & 7.42 & -.29 & - & 1.79 & -1.29 & - & - & - & - \\
\hline Brazil & 14.83 & .57 & .12 & 2.19 & -.43 & 4.01 & 4.34 & 3.99 & 2.71 \\
\hline Canada & 12.00 & .97 & .83 & 2.66 & 1.17 & 3.21 & 3.38 & 3.85 & 1.96 \\
\hline Chile & 14.33 & .13 & - & 2.41 & -.37 & 2.98 & 3.87 & 3.85 & 2.03 \\
\hline China & 8.33 & -1.92 & -.22 & 1.27 & -1.30 & 2.35 & 3.65 & 3.58 & 2.07 \\
\hline Egypt & 8.00 & -.78 & - & 1.66 & -1.40 & - & - & - & - \\
\hline Finland & 14.33 & .74 & -.97 & 2.90 & .99 & 3.25 & 3.94 & 3.29 & 2.52 \\
\hline Germany & 15.67 & .18 & -.47 & 2.56 & 1.00 & 3.37 & 3.72 & 3.02 & 2.05 \\
\hline $\begin{array}{l}\text { Hong Kong, } \\
\text { China (SAR) }\end{array}$ & 12.33 & - & 1.74 & - & .74 & - & - & - & - \\
\hline India & 9.67 & -1.13 & 1.60 & 1.73 & -.70 & - & - & - & - \\
\hline Israel & 11.33 & -.18 & - & 2.12 & .50 & - & - & - & - \\
\hline Japan & 9.17 & -.86 & .96 & 2.34 & .73 & 2.62 & 3.03 & 3.28 & 2.00 \\
\hline Jordan & 7.33 & -.77 & - & 1.60 & -.94 & - & - & - & - \\
\hline Malaysia & 8.33 & .08 & - & - & -.54 & - & - & - & - \\
\hline Mexico & 14.17 & -.28 & -.15 & 2.36 & -.24 & - & - & - & - \\
\hline New Zealand & 13.83 & .82 & -.33 & 2.43 & 1.00 & - & - & - & - \\
\hline $\begin{array}{l}\text { Papua } \\
\text { New Guinea }\end{array}$ & 8.17 & - & - & - & -.40 & - & - & - & - \\
\hline Poland & 11.67 & -.90 & - & 1.91 & -.25 & 3.66 & 3.55 & 3.65 & 3.58 \\
\hline
\end{tabular}




\begin{tabular}{|c|c|c|c|c|c|c|c|c|c|}
\hline \multirow[b]{2}{*}{ Nation } & \multirow[b]{2}{*}{$\begin{array}{c}\text { Mean ranking } \\
\text { score of SFC }\end{array}$} & \multirow[b]{2}{*}{ Happiness } & \multirow[b]{2}{*}{ Depression } & \multirow[b]{2}{*}{ Postmaterialism } & \multirow[b]{2}{*}{ BNFIt } & \multicolumn{4}{|c|}{ Motives for voluntary work } \\
\hline & & & & & & $\begin{array}{c}\text { 'Personal } \\
\text { satisfaction' }\end{array}$ & $\begin{array}{c}\text { 'Good } \\
\text { experience' }\end{array}$ & $\begin{array}{c}\text { 'Contribution to } \\
\text { community' }\end{array}$ & $\begin{array}{c}\text { 'Could } \\
\text { not } \\
\text { refuse' }\end{array}$ \\
\hline Romania & 9.33 & - & - & 1.81 & -.79 & 2.91 & 2.80 & 2.73 & 2.33 \\
\hline Singapore & 13.67 & .43 & - & 1.95 & -.05 & - & - & - & - \\
\hline Slovakia $†$ & 10.00 & - & - & 1.79 & .13 & 2.85 & 3.98 & 3.93 & 2.47 \\
\hline Slovenia† & 8.50 & - & - & 2.25 & .35 & 2.64 & 3.25 & 3.46 & 1.47 \\
\hline South Africa & 11.67 & -.63 & - & 1.70 & -.64 & - & - & - & - \\
\hline Spain & 13.83 & -.41 & -.31 & 2.58 & .60 & 3.27 & 3.05 & 3.27 & 1.50 \\
\hline Sweden & 14.83 & 1.03 & -.45 & 2.60 & 1.22 & 2.30 & 3.92 & 3.21 & 1.77 \\
\hline Taiwan & 14.42 & - & - & 1.52 & .01 & - & - & - & - \\
\hline Turkey & 13.00 & -1.02 & 1.64 & 2.32 & -.75 & - & - & - & - \\
\hline Ukraine & 11.42 & - & - & 1.45 & -.62 & - & - & - & - \\
\hline $\begin{array}{l}\text { United } \\
\text { Kingdom }\end{array}$ & 13.67 & .69 & .02 & 2.42 & .99 & 2.87 & 2.95 & 3.77 & 1.72 \\
\hline United States & 10.17 & .91 & -.11 & 2.31 & 1.23 & 3.18 & 3.06 & 3.91 & 2.05 \\
\hline Vietnam & 8.00 & - & - & - & -1.33 & - & - & - & - \\
\hline Mean & 11.49 & -.07 & .25 & 2.12 & -.075 & 3.03 & 3.50 & 3.52 & 2.15 \\
\hline SD & 2.66 & .82 & .84 & .44 & .86 & .46 & .46 & .38 & .53 \\
\hline
\end{tabular}

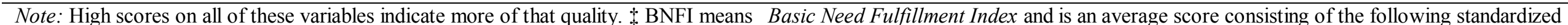

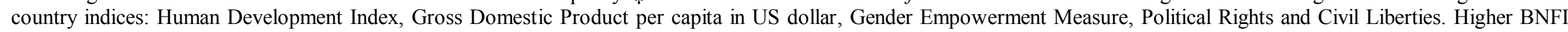
scores denote higher fulfilment of these basic needs. $\dagger=$ Mean rank for these countries is based on a subset of $S F C$ values. 


\section{Results}

Since I have directional hypotheses, the results reported in this section are based on one-tailed statistical tests.

\section{Correlational Analyses}

SFC and Schwartz' Values. The Spearman correlations between SFC and Schwartz' cultural value types confirmed the hypothesized sinusoidal pattern (see Figure 3.2) with significant positive correlations for Autonomy values (Intellectual Autonomy: $r_{s}=.39, p<.05$, Affective Autonomy: $\left.r_{s}=.36, p<.05\right)$ as well as Egalitarianism $\left(r_{s}=.45, p<.01\right)$ and Harmony $\left(r_{s}=.36, p<.05\right)$. There were significant negative correlations for Embeddedness $\left(r_{s}=-.56, p<.001\right)$ and Mastery $\left(r_{s}\right.$ $=-.31, p<.05)$, but not for Hierarchy $\left(r_{s}=-.21, p=.13\right)$. The correlations support in general the location of SFC in Schwartz' value circle as displayed in Figure 3.1. SFC is compatible with cultural values that lay emphasis on the individual and is conflicting with the more 'group-focused' values. 


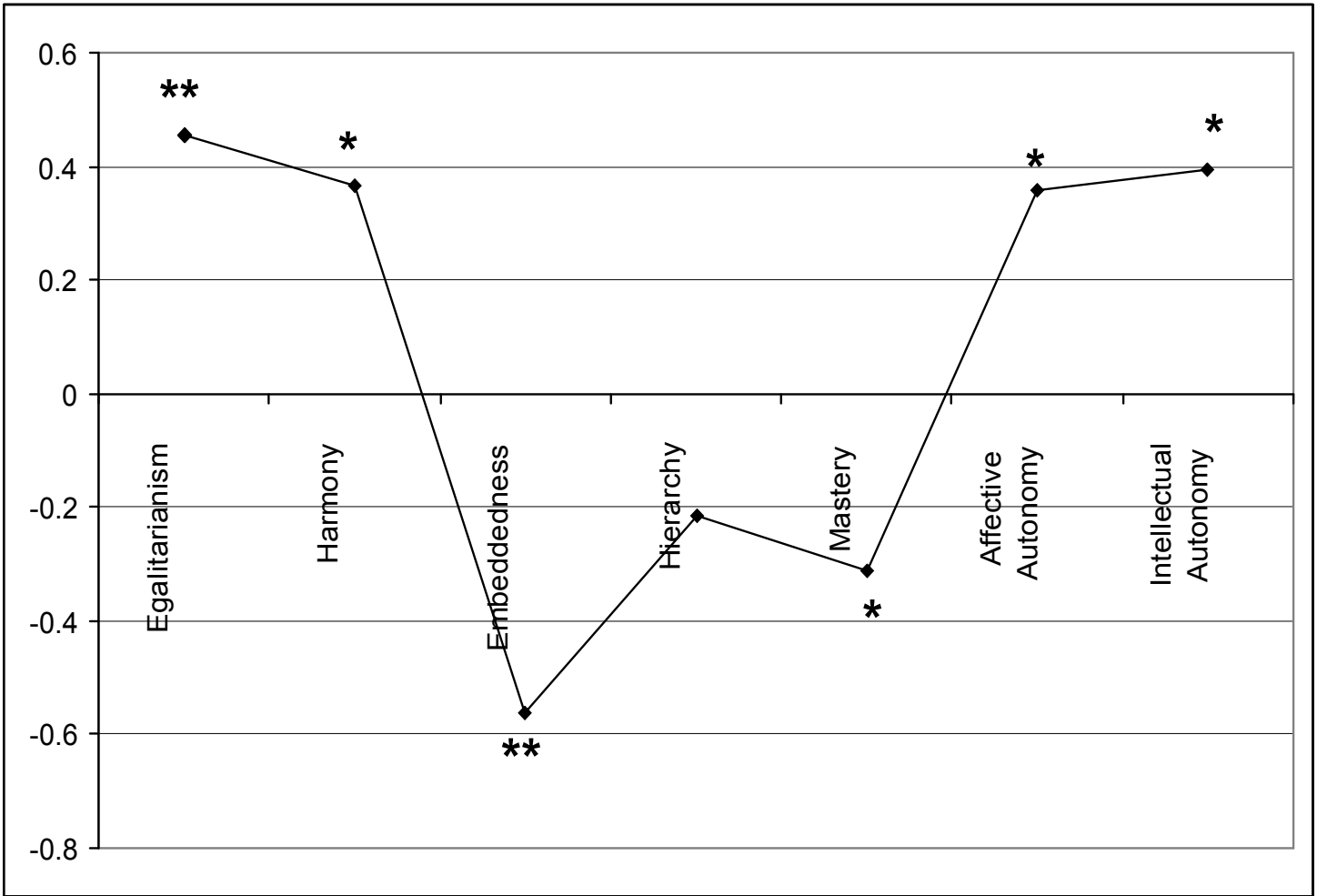

Figure 3.2: Spearman correlations between average ranking of SFC and Schwartz' culture-level value types Note: ${ }^{*} p<.05,{ }^{* *} p<.01$, one-tailed.

SFC and External Validity Measures. Concerning the relationship between SFC and Subjective Well-being, I found the hypothesized significant positive correlation $\left(r_{s}\right.$ $=.59, p<.001)$. The correlation with the Depression scores on the BDI shows a relationship of similar strength, but as hypothesized in the opposite direction $\left(r_{s}=-.54\right.$, $p<.05)$. As predicted, I found a correlation between $S F C$ and postmaterialism that is significantly positive $\left(r_{s}=.61, p<.001\right)$, meaning that a priority on $S F C$ is related to an emphasis on non-material goals. I also found that intrinsic motives to engage in prosocial behaviour are related to $S F C$. There was a significant positive correlation between $S F C$ and the reason for doing voluntary work because of 'personal satisfaction' $\left(r_{s}=.53, p<.05\right)$ and because of the opportunity to 'gain new skills and useful experience' $\left(r_{s}=.48, p<.05\right)$. As hypothesized, $S F C$ was unrelated to non-autonomous or extrinsic motives for engaging in voluntary work which was tested with the items 
'did not want to but could not refuse' $\left(r_{s}=-.04, p>.05\right)$ and 'making a contribution to the local community' $\left(r_{s}=.08, p>.05\right)$. These findings support the discriminant validity of $S F C$.

Regarding the question which societies tend to endorse $S F C$, I found the trend that countries with higher level of basic need fulfilment showed higher endorsement of $S F C$ values $\left(r_{s}=.60, p<.001\right)$. I also looked into the correlations of all other Rokeach value types with BNFI. The finding is that $S F C$ is the only value type that shows this strongly positive relationship. The Egalitarianism/ Harmony type was only marginally significant $\left(r_{s}=.24, p<.10\right)$ and the Autonomy value type did not reach statistical significance when correlated with BNFI $\left(r_{s}=.09, p>.05\right)$. There were significant negative correlations with Embeddedness $\left(r_{s}=-.46, p<.01\right)$ and with Mastery $\left(r_{s}=-\right.$ $.62, p<.001)$.

In order to test Hypothesis $5 \mathrm{~b}$, i.e. to find out whether the relationship between BNFI and SFC applies only to Western countries, or is also replicable in non-Western nations, I split the dataset accordingly. Fourteen countries were identified as belonging to the West (Anglo-Saxon countries and Europe) and eighteen countries as pertaining to non-Western countries (Asia, Africa, Middle East, and South-America). I computed the correlations between $S F C$ and BNFI for each sub-sample again. I found moderate correlations for both the Western $\left(r_{s}=.53, p<.05\right)$ and non-Western sub-sample $\left(r_{s}=\right.$ $.60, p<.01)$. These results support Inglehart's (1997) proposition: the higher the level of socio-economic development of countries, the greater is the tendency to pursue nonmaterial goals, independent of cultural region. Hence, it is not a uniquely Western phenomenon, but holds in non-Western societies, too ${ }^{10}$.

\footnotetext{
${ }^{10}$ See also Appendix B for a figure illustrating the distribution of countries regarding their socioeconomic development and importance of $S F C$ values.
} 
Regression Analyses

I ran hierarchical multiple regression analysis to test whether $S F C$ adds unique explained variance after controlling for the Autonomy value type. The finding was that $S F C$ significantly accounted for unique variance, except for the case where Depression was the dependent variable $(\Delta F(1,20)=.95, p>.05, \beta=-.25)$. It can be concluded that $S F C$ possesses incremental validity as a predictor for $\operatorname{SWB}(\Delta F(1,20)=5.03, p<.05, \beta$ $=.43)$, for postmaterialism $(\Delta F(1,24)=6.30, p<.05, \beta=.39)$, for intrinsic motives of pro-social behaviour ('personal satisfaction'- item: $\Delta F(1,12)=7.10, p<.05, \beta=.67$; 'useful experience'- item: $\Delta F(1,12)=10.27, p<.01, \beta=.74)$ and for BNFI $(\Delta F(1,26)$ $=5.28, p<.05, \beta=.31$ ), after controlling for Autonomy in the first step.

\section{Discussion}

\section{Summary}

I validated the location of $S F C$ in the value circle by correlating it with Schwartz' data. SFC is compatible with Autonomy, Egalitarianism and Harmony, and conflicting with Embeddedness, Hierarchy, and Mastery. I further tested the relationship of $S F C$ with a number of external variables. $S F C$ is related to higher levels of Subjective Well-being and lower levels of Depression in a society. I also found that $S F C$ is correlated with postmaterialism values indicating that it is a pursuit of nonmaterial goals. Regarding Waterman's (1981) theoretical suggestions, that individualism may imply interrelatedness and express itself in pro-social behaviours motivated by the intrinsic satisfaction to help others, I found indeed evidence that a priority of $S F C$ was related to engagement in voluntary work motivated by intrinsic reasons, and not by social obligations. The motive of helping behaviour corroborates my assumption that despite its relational component, $S F C$ belongs to an individualistic value orientation. I also tested the scarcity hypothesis, as proposed by Inglehart (1997), 
that the fulfillment of basic needs in a society gives way to the pursuit of non-material goals. While I cannot test causality, I found at least a significant correlation between the level of basic need fulfillment in a country and the importance of $S F C$ values. Also consistent with Inglehart, I was able to show that this phenomenon is not unique to Western countries. I tested the incremental validity of $S F C$ by assessing its potential to account for unique variance after controlling for the Autonomy value type. I found the incremental validity of $S F C$ in all cases confirmed, except for Depression (which may be due to the smaller number of countries included in that analysis, see Table 3.4).

\section{What is the Societal Meaning of SFC?}

What does $S F C$ mean for societies from a theoretical point of view? What are the basic issues with which each society is confronted and for which it needs to find a solution? I have so far interpreted $S F C$ as an individualistic value orientation that is closely related to Autonomy. There were a number of reasons that guided me to interpret it that way and that I also found empirically confirmed: $S F C$ is located very closely to Autonomy and clearly opposite to Embeddedness in the MDS. Furthermore, it contains a number of self-focused values and is empirically related to self-focused variables. Moreover, I did not find a new opposite value type to $S F C$ which could have been interpreted as an entirely new value dimension. Taken all this together, it led me to conclude that $S F C$ is a value type that is complementary to Autonomy. From this it follows that it relates to the same basic societal issue as Autonomy and Embeddedness, i.e. to the question of the boundaries between the person and the group.

While Schwartz proposed that relationships play a major role in Embeddedness cultures, they do not seem to be important for Autonomy-oriented societies. Yet, establishing relationships with others is an important universal need (cf. Ryan \& Deci, 2002). I suggest that some societies may achieve it with embedding their members into 
social groups (as in Embeddedness cultures), other societies may compensate the lack of social networks by emphasizing the value of independence and hedonistic experiences (as in Autonomy cultures) or by leaving it upon the individual to discover the intrinsic value of relationships (as in Self-fulfilled Connectedness cultures). But what is then the difference of relationships in 'embedded' and 'connected' cultures?

In 'embedded' cultures, there is a social structure that assures that individuals are cared for by automatically belonging to an established social group. There is not much choice: the social expectation tends to fit into the pre-established social fabric with all its accompanying obligations. Thus, a sense of meaning and purpose in life may be derived from social relationships and from identifying with the group, but this is rather imposed by society than based on free choice. Consequently, there may be a lesser awareness of the positive impact that social relationships have for the self. The great sense of personal gratification derived from relationships may be less accentuated in 'embedded' than in 'connected' societies. In Self-fulfilled Connectedness societies on the other hand, the society provides the freedom for individuals to engage in discretionary relationships that in turn provide a sense of meaning and satisfaction. The resulting positive feelings of happiness and well-being derived from voluntary relationships provide the underlying motivation to pursue this value type when relatedness is not 'institutionalized' in a society.

I provided empirical evidence that $S F C$ is a meaningful value type. Based on the current evidence, I can conclude that Schwartz' approach in constructing the SVS and conducting the culture-level analysis may have led to its omission. It would be of great interest to include these kinds of values in further studies. There is already empirical evidence at the individual-level that autonomy and relatedness are indeed compatible and not conflicting (for a review see, Kağıtçıbaşı, 2005). Studies that examine this phenomenon with the inclusion of $S F C$ values in their value inventories, may certainly 
give an extended insight into the system of values and its meaning for both individuals and societies.

\section{General Discussion}

\section{Evaluation of the Replication}

The main purpose of this study was to replicate Schwartz' culture-level value types by conducting a meta-analysis on the RVS. By deriving a MDS on Rokeach values and using the configurational verification approach, I was able to identify four out of six of Schwartz' cultural value types. Some of the value types were not clearly separable, probably because of item under-representation in the respective value clusters (i.e. in Harmony and in Affective Autonomy). I assessed the degree of similarity between the RVS- and SVS-MDS more precisely by correlating the coordinates of matching values and found significant correlations of moderate sizes. The question remains whether the replication can be regarded as successful. I suggest that two factors need to be taken into account to answer this question: (a) the plausibility of the MDS; and (b) the level of imprecision in relation to the original study. Schwartz' theory of cultural dimensions is based on the assumption that every society needs to solve universally occurring issues. The solutions to these issues take the form of value orientations with conflicting poles. The RVS-MDS confirms an approximate structure of these conflicting value types. Despite the merging of some value clusters, the MDS shows a plausible result: only value types that are compatible, and thus share some degree of similarity, merge and this is probably due to item under-representation and the absence of Hierarchy values. Given the fact that the comparison is based on a rather limited set of 23 or 28 values respectively, it is encouraging to have been able to replicate a rough structure of culture-level value types. 
Regarding the level of imprecision to the original study, the present study can be regarded as 'fairly imprecise'. Schwartz' (1994a, 2006) samples consisted of university students and teachers, whereas the meta-analysis included diverse samples such as working adults, and samples from the general population. The sampled cultural groups reflect also a somewhat different distribution in terms of geographical regions they belong to, compared to Schwartz' first culture-level analysis (see Schwartz, 1994a). The response format of the RVS is entirely different to the SVS (ranking vs. rating). Finally, the time frame for which data is available is larger for the RVS dataset than for the SVS dataset. In regard of all these differences, I think that the replication can be considered as successful. The similarity of the Schwartz- and Rokeach values-MDS results, despite the high degree of 'imprecision' in the replication, can also be interpreted as a strength in terms of the robustness of Schwartz' theory.

\section{Limitations}

There are a number of limitations in this study. A major limitation is that I needed to average items across different decades to enhance the sample size of countries. In this way I ignored temporal effects, such as value changes, that may occur within societies. For some countries, data on the Rokeach values were only available for specific years or different time frames than for other countries. I tried to address this issue for the correlational analyses in Study $1 \mathrm{~b}$. I aimed to obtain scores for the external variables that match the large time frame of the RVS-dataset. In some cases this was not possible (e.g., pro-social behaviour items from the World and European Value Survey). This mismatch of time frames of country scores may be one explanation why I consistently found correlations of only moderate sizes and not higher. However, there are probably a number of other factors that may have lowered the correlations which are difficult to identify or which I could not control. Yet, the fact that I still found 
significant correlations in the hypothesized direction suggests that the relationships are relatively stable. Countries from Eastern Europe, for example, experienced great political and socio-economic changes. Yet, I found a consistent result: they scored relatively low on the Basic Need Fulfilment Index and also showed relatively low priority on the $S F C$ value type. This finding still emerged as significant even if the country scores were averaged across almost four decades, or if some data points were missing, such as in the case of Slovenia and Slovakia. Despite all these shortcomings, there is theoretical and empirical evidence that $S F C$ is a meaningful value type at the culture-level.

Another major limitation is that I was not able to examine the value structure at the individual-level. The meta-analytical dataset for the Rokeach values consists of mean or median rank orders for samples. In most of the cases the number of samples within countries is relatively low which does not allow conducting analyses at the sample-level. On the other hand, the aim was to replicate Schwartz' culture-level value structure and not to examine the individual-level value structure. I showed that the RVS- and SVS- culture-level value structure were significantly related to each other despite a number of dissimilarities between both datasets, such as sample characteristics, sampling time frame, etc.

\section{Conclusion}

The emergence of a new finding is rather unusual when conducting replications. This may also be the reason why replication studies are often felt to be redundant and as not adding new knowledge or advancing the understanding of an issue. New findings are commonly seen as more informative and interesting, even if they are of unknown reliability and generalizability (Neuliep \& Crandall, 1993). The present study meets both objectives: ensuring the generalizability of Schwartz' theory and adding new 
knowledge. I successfully replicated the structure of values at the culture-level and at the same time I was able to identify a new value type which I labelled Self-fulfilled Connectedness. I hope that this discovery stimulates further studies to explore this particular value type and its meaning to individuals in different cultures. 


\section{CHAPTER FOUR: STUDY 2}

\section{Do Cultural Values Explain Individuals' Moral Attitudes?}

\section{A Cross-cultural Multi-level Approach}

\section{Introduction}

Values - including cultural values - are seen as the abstract motivations that guide, justify, and explain individuals' attitudes (Feather, 1985; Rokeach, 1973; Schwartz, 2007c). But do cultural values also relate to moral attitudes? This question, as simple as it appears, taps into a major controversy of whether morality is universal or culturally determined. Moral universalists (e.g. Kohlberg, 1984; Piaget, 1932/1965; Rawls, 1999; Turiel, 2002) posit that issues of justice and fairness are the common cornerstones of morality. Evolutionary theorists explain these universal moral norms with the adaptive benefit of social cooperation (see Krebs, 2008, for a review on morality and evolutionary theory). As expressed by Rawls (1999): "social cooperation makes life better for all than any would have if each were to live solely by his own efforts" (p. 4). From an evolutionary perspective, people who engage in unfair behaviour and thus take advantage of the system of social cooperation are judged as wrongdoers - regardless of the cultural context.

Note. An earlier version of this chapter has been submitted for publication to the European Journal of Social Psychology and is hereafter referenced as follows: Vauclair \& Fischer (2009). Do cultural values explain individuals' moral attitudes? A cross-cultural multi-level approach. Manuscript under review. 
In contrast, cultural psychologists (e.g. Haidt, et al., 1993; Miller, 2001; Shweder, et al., 1987) argue that judgments of right and wrong are influenced by culturally based conceptions of the self. People who live in societies in which they regard themselves as rather independent from the social group adopt the moral discourse of Autonomy (Shweder, et al., 1997). What is right or wrong depends on whether it affects individual rights and freedoms and has been called a rights-based morality (Chiu, et al., 1997; Dworkin, 1978). In a moral discourse of Community, however, individuals perceive themselves as interconnected with the social group and judge right and wrong on the basis of interpersonal duties, social roles and obligations - called a duty-based morality.

Previous research on these two very different positions of morality has been mostly qualitative (e.g. Turiel, 2002; Shweder, et al., 1997) and based on comparisons between only a few cultural groups (e.g. Haidt, et al., 1993; Miller, et al., 1990; Nucci, et al., 1983; Shweder, et al., 1997). In the present study, I examined commonalities and differences in morality across a large number of countries. I conducted an analysis of the Morally Debatable Behaviour Scale (MDBS, Harding \& Phillips, 1986) included in the European and World Value Survey (EVS/ WVS). The scale maps onto two different theories of morality by measuring individuals' attitudes towards (1) cheating behaviours which violate the code of social cooperation, and (2) behaviours involving choices in the personal and sexual domain, such as divorce, abortion and prostitution.

In order to rigorously test whether any observed differences between countries are indeed due to cultural effects, I adopted two strategies recommended for advanced cross-cultural research (see e.g. Matsumoto \& Yoo, 2006). First, I unpackaged culture, i.e. I used specific variables as a measure of culture in the form of Schwartz' cultural values (Schwartz, 1994b, 2006). Secondly, I employed a multilevel model in which I tested whether cultural values accounted for individuals' moral attitudes. This was done 
after controlling for individual differences and economic development, as a contextual factor, to rule out the possibility that these non-cultural variables contribute to any observed country differences. To the best of my knowledge, this is the first study to use such a comprehensive statistical model to shed light on both cultural similarities and differences in morality.

In the following paragraphs, I will provide a brief summary of past research using the MDBS. I will then outline relevant theories tapping into the moral universalism and relativism debate from which I derive the hypothesis.

\section{The Morally Debatable Behaviour Scale (MDBS)}

\section{A Measure of Moral Attitudes}

The initial Morally Debatable Behaviour Scale was developed in the 1980's and consisted of 22 items (Harding \& Phillips, 1986). The purpose of the scale was to assess the justifiability of behaviours reflecting moral issues (e.g. divorce) which people may confront in their lives or have an opinion about. There are no test-retest indices or reliability coefficients reported (see also Braithwaite \& Scott, 1991). The items have been interpreted as measuring values in general (Braithwaite \& Scott, 1991; Inglehart, 2006), moral values in particular (e.g. Halpern, 2001; Nevitte \& Cochrane, 2006), moral beliefs (e.g. Halman, 1996) or social attitudes (e.g. Schwartz, 2006b). This conceptual conflation of attitudes and values impedes a deeper understanding of the relationship between these two concepts, such as whether people's attitudes can be predicted from their cultural values. Value theorists have differentiated attitudes from values by defining attitudes as evaluative judgments of specific entities and values as abstract beliefs about desirable goals, ordered by relative importance, that guide individuals as they evaluate events, people, and actions (Rohan, 2000; Rokeach, 1973; Schwartz, 1992). 
Attitudes elicit affective, behavioural and cognitive responses (Rosenberg \& Hovland, 1960). In the case of the MDBS, the items are rated on a scale ranging from 'never justified' to 'always justified'. It can be said that these items elicit a cognitive response since individuals express their beliefs about specific behaviours related to morality. I conceptualized the scale as a measure of attitudes. The response to the items can also be called a moral judgment ranging from more lenient to stricter judgments. In regard to the MDBS, I did not draw a distinction between the terms moral attitude and moral judgment. For the present study, they both refer to an individuals' evaluation of a moral issue as rather permissible and tolerable or not.

\section{The Factor Structure}

Harding and Phillips (1986) reported the most comprehensive analysis on the scale using data from the EVS carried out in 1981 in ten Western European countries. They found that the scale typically yielded a three factor structure distinguishing moral issues into a (1) personal-sexual morality with a focus on matters of life and death, as well as sexual relations, a (2) self-interest (or honest-dishonest) morality focusing on issues of personal integrity and honesty, and a (3) legal-illegal or (legal-punitive) morality defined by behaviours which are regulated by law. The authors also reported that items tapping into the personal-sexual domain loaded relatively clearly on a single factor. The other two factors had some cross-loading items (e.g., 'avoiding a fare on public transport' which may be expected as it is both 'dishonest' and 'illegal'), raising the question of whether a more parsimonious two-factor structure would not fit individuals' perception of moral issues better than a three-factor structure. Furthermore, the cross-cultural validity of the factor structure was relatively consistent within all Western European countries (Harding \& Phillips, 1986); however, it still needs to be shown whether this also applies to non-European countries which exhibit very different 
cultures compared to Europe, and in particular when equivalence in factor structures is evaluated with more rigorous tests such as confirmatory factor analysis.

\section{Country-level Predictors of Individuals' Moral Attitudes}

The Moral Universalism Account: Cheating is Wrong - Everywhere

According to the universalistic paradigm (e.g. Kant, 1785/1994; Kohlberg, 1984; Piaget, 1965; Rawls, 1999; Turiel, 2002), morality is regarded as unrelated to the social or cultural context. Empirically, there are particular aspects of morality which have been found to be relatively invariant across cultures. This includes intentional acts with intrinsically harmful consequences to others, such as stealing or hitting, which are universally judged as wrong and as moral matters (Turiel, 1983, 2002). While this research gives some insight into cross-cultural commonalities, it does not provide an explanation why there is such a universal sense of justice and fairness. Yet, it seems paramount to uncover the underlying mechanism of morality in order to understand, explain and further investigate its manifestation in human behaviour. Theorists attempting to explain morality often adopt evolutionary theories (e.g. Axelrod, 1984; Cosmides \& Tooby, 1992; Dawkins, 1976; Hamilton, 1964; Trivers, 1971) which provide an intriguing framework from which predictions on human morality can be derived.

The main argument of evolutionary theory is that the driving force of evolution consists of individuals trying to maximise the transmission of their genes to the next generation (Darwin, 1874; see Krebs, 2008). For the human species, this is facilitated through altruism and cooperation. In the ancestral environment, social cooperation in the form of group hunting or protection from predators has increased the likelihood of survival for both the individual and the whole group (Krebs, 2005; Krebs, 2008; Wright, 1996). Thus, a genetic disposition for a moral code of social cooperation has been 
transmitted to following generations and finally persisted as a human universal until today. Without this sense of morality, it would be difficult for members of a social group to coordinate their behaviours in a way that maximizes the benefits for themselves and the group as a whole. The regulatory force for the development of social cooperation has presumably been reciprocity (Gouldner, 1960): cooperation is rewarded with cooperation (Axelrod \& Hamilton, 1981). In contrast, selfishness and cheating in the form of free riding and rule breaking are ultimately sanctioned socially (Broom, 2003) or legally (cf. Harding and Phillips, 1983) - even if people are not personally affected by the act (Price, Cosmides, \& Tooby, 2002). Hence, there is a deep universal sense of justice that also involves a desire to condemn and even punish wrongdoers (Wilson, 1993). Evolutionary theorists propose that this universal moral norm evolved because it is an effective mean of upholding mutually beneficial cooperative relations (Krebs, 2005).

The dishonest and illegal factor of the MDBS may be interpreted as capturing issues of selfishness and breaches of social cooperation (e.g., 'cheating on taxes' or 'avoiding a fare on public transport'). Considering the importance of social cooperation in the ontogenetic development of humans, I expected that attitudes towards these kinds of behaviours are not determined by culture or cultural values. This does not mean that there may be no individual differences or within-country variation respectively. Even in the framework of evolutionary psychology it is acknowledged that there are individual differences in adherence to moral codes with some individuals trying to take advantage of others (cf. Krebs, 2008). However, given the importance of social cooperation for the cohesion of all social groups, I assumed that it would be dysfunctional for any society to endorse cultural values which promote these kinds of behaviours. Hence, I expected cultural values not to be significant predictors of individuals' attitudes towards dishonest and illegal behaviours. 
The Moral Relativism Account: Right or Wrong Choices?

According to the relativism paradigm, morality is seen as culturally influenced (e.g. Haidt, et al., 1993; Miller, 2001; Shweder, et al., 1987). Empirically it has been shown that this applies specifically to issues that are related to a culturally based conception of the self (e.g. Shweder, et al., 1997). The psychological dichotomy of independent and interdependent selves originally coined by Markus and Kitayama (1991) is here relevant. Miller et al. (1990), for example, found that Hindu-Indians, who endorse an interdependent conception of the self, tended to view 'helping strangers' as an interpersonal responsibility and therefore as a moral matter. In contrast, for European-Americans who tend to endorse an independent conception of the self, 'helping strangers' was affected by personal preferences and non-moral considerations such as liking the stranger. They also thought that greater satisfaction is linked to voluntary, as opposed to role-based helping. Hindu-Indians assumed that individuals experience the same satisfaction in both cases.

The moral discourses that goes along with these different cultural conceptions of the self have been labeled ethic of Autonomy versus ethic of Community (Shweder, et al., 1997) or a rights-based versus duty-based morality respectively (Chiu, et al., 1997; Dworkin, 1978). A rights-based morality is more person-centred by focusing on individual rights, including the right to pursue own interests and personal goals for the sake of one's own personal well-being. An act is only judged as wrong if it causes harm or restricts another person's freedom or rights (Shweder \& Haidt, 2000). A duty-based morality on the other hand is more system-oriented and serves to maintain the status quo in a social group (Chiu, et al., 1997). Fulfilling social obligations is of supreme importance. A person's choices and actions, such as whom to marry, are not seen as only affecting the individual but also closely related others. Hence, these decisions can 
be cause of shame and dishonour and can be judged as wrong if the interests of the community are disregarded.

Whether a society lays more emphasis on the individual or the social group or on Autonomy versus Community respectively can be empirically assessed with Schwartz' (1994) cultural value orientations Autonomy versus Embeddedness. Autonomy values represent an emphasis on being independent, with the right to pursue one's own selffulfilment, be it in an intellectual or affective way. Embeddedness values, however, resemble a duty-based moral orientation. They emphasize the importance of social relatedness, the traditional order and the maintenance of the status quo. This bipolar value orientation may also be regarded as paralleling the classical individualism and collectivism distinction (Inglehart, 2006; Kağıtçıbaş1, 1997) originally put forward by Hofstede (1980). Since the personal-sexual items from the MDBS draw on behavioural choices that are directly related to a person's own life, I expected that countries endorsing Autonomy rather than Embeddedness provide a more lenient context in regard to these issues which in turn allows individuals to form more lenient attitudes ${ }^{11}$. Thus, I hypothesized that:

In countries characterized by an Autonomy value orientation, individuals show a more lenient attitude towards personal-sexual issues than in countries characterized by an Embeddedness value orientation.

\footnotetext{
${ }^{11}$ Additionally to the Autonomy/ Embeddedness dimension, Schwartz $(1994,2006)$ proposed two further bipolar cultural value orientations: Hierarchy vs. Egalitarianism and Harmony vs. Mastery. Relationships of these additional cultural values with individuals' personal-sexual attitudes was not expected. However, they were included in the regression model to examine whether they contributed to the explanation of moral attitudes. Therefore, it was tested whether Autonomy vs. Embeddedness is the active ingredient of culture, as predicted.

Figure 6.1 in chapter five shows a co-plot of countries on these cultural value orientations.
} 


\section{'Control Variables'}

Economic Development of Countries

Between-country differences in moral attitudes may not only occur because of cultural differences, but also because of 'non-cultural' variables such as economic factors. Affluence has often been found to be closely related to cultural values assessing individualism and collectivism (e.g. Hofstede, 1980; see also Inglehart \& Welzel, 2005; Triandis, 2001). Schwartz (2006) reported correlations as high as .73 between GDP per capita and the Autonomy-Embeddedness value dimension. Furthermore, time-series analyses have shown that, with economic growth, societies tend to move from selfconstraining values to more secular-rational and self-expressive ones (Inglehart \& Baker, 2000; Inglehart \& Welzel, 2005). Hence, a country's increasing affluence may provide a context in which individuals form more permissive moral attitudes. Given the considerable link between cultural values and the socio-economic development of countries found in past research, I examined the extent to which affluence operates as a predictor of moral attitudes. Since economic factors belong to the contextual variables that are usually regarded as non-cultural (cf. Matsumoto \& Yoo, 2006), I controlled for it in the regression analysis when investigating the unique contribution of cultural values. In this way I was able to examine whether any of the observed between-country differences occur because of economic factors only or whether cultural values add incremental validity in the prediction of moral attitudes. 


\section{Individual-level Correlates of Moral Attitudes}

The most comprehensive study of individual-level differences in moral attitudes has been conducted by Harding and Phillips (1986) using data from ten Western European countries. The authors concluded that older age, religiosity and conservative political beliefs were all associated with greater strictness on moral issues. They did not find strong gender differences, but pointed out that women emerged as slightly stricter in their moral judgment. There were also differences in associations between the content of moral issues and individuals' socio-economic status: the higher individuals' income, their social class and their level of education, the more lenient they judged personalsexual issues. This relationship was less consistent in regard to the judgment of dishonest and illegal behaviours.

It is unclear whether these relationships hold when non-European countries are examined. The findings nevertheless provide a rationale for controlling for certain individual-level predictors when aiming to explain between-country variation in moral attitudes. Hence, I examined whether socio-demographic variables (age, gender, religiosity, political orientation and socio-economic status) predicted moral attitudes. I also investigated whether the relationship between socio-demographics and moral attitudes varies across countries and might be moderated by context variables. For a better overview, the multilevel model with all hypothesized variables is depicted in Figure 4.1. 


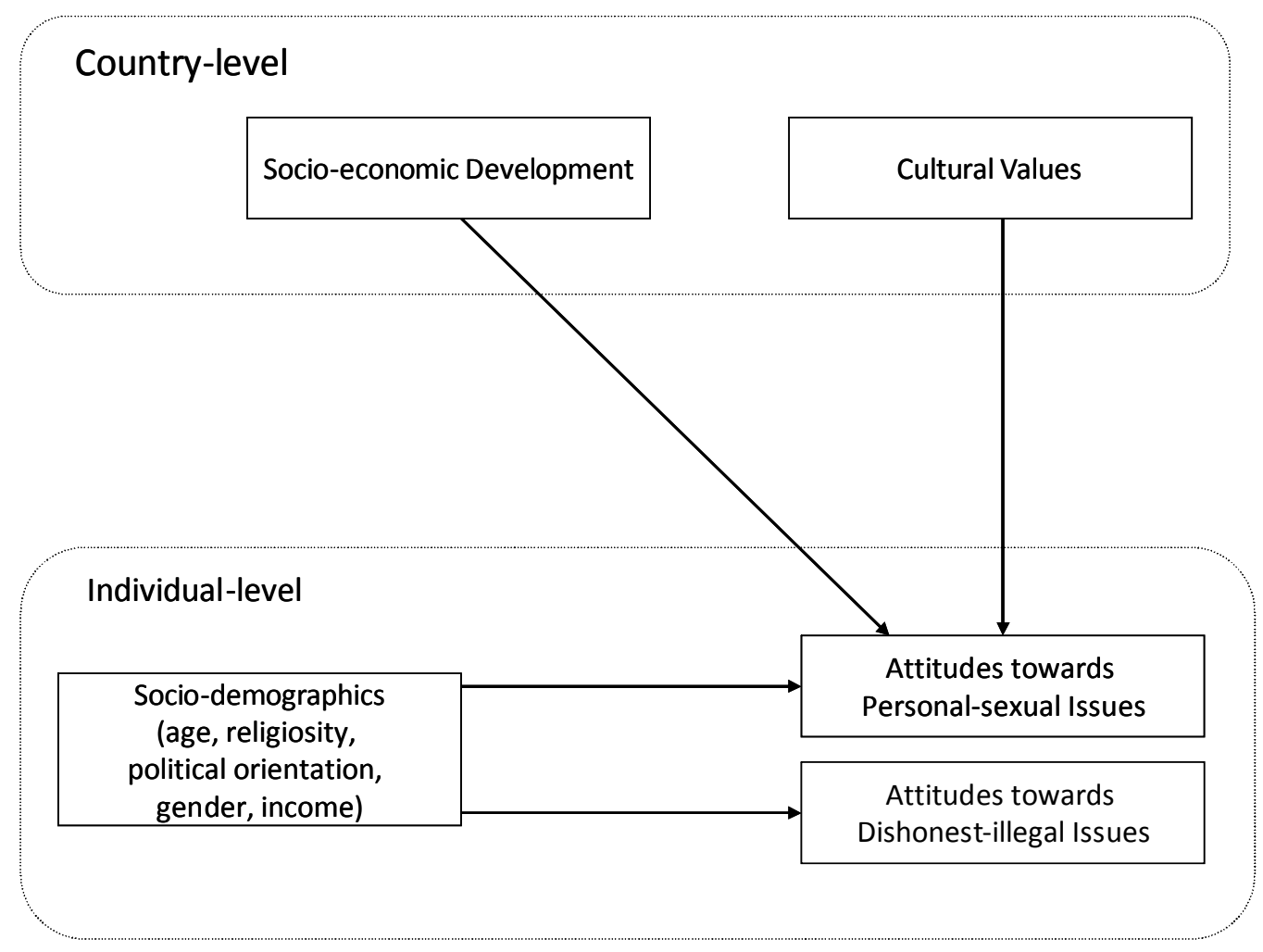

Figure 4.1: Hypothesized multilevel model for the prediction of individuals' moral attitudes.

\section{Method}

\section{Instruments and Data}

\section{Moral Attitudes as Dependent Variables}

To assess individuals' moral attitudes across different countries, I used data on the MDBS gathered through the European and World Value Survey (EVS/ WVS) as available from the World Value Survey webpage ${ }^{12}$. The WVS together with the EVS is carried out by a worldwide network of social scientists studying value change. All samples are representative within a country in terms of age, sex, occupation and regional distribution. Respondents are interviewed using a standardized questionnaire with a number of questions in regard to religion, gender roles, work, politics, attitudes towards other groups, environmental protection and subjective well-being.

\footnotetext{
${ }^{12}$ Retrieved October 2008, from http://www.worldvaluessurvey.org/. Note that all details regarding the survey and samples are on this website.
} 
I used survey data on moral attitudes from four study waves $(1981-1984,1989$ - 1993, 1994 - 1998 and 1999 - 2004). A number of items have been added to the MDBS until the 2004 study wave, so that the total scale consists of 31 items; however only ten of them have been used consistently across all study waves and countries. Therefore, I used only these ten items for the present analyses (see Table 4.1 in the results section for the content of items). The items are rated on a 10-point Likert scale with lower numbers reflecting a more severe attitude towards the issue in question $(1=$ 'never justified') and higher numbers a more lenient or tolerant attitude (10 = 'always justified'). Since my focus in this study was on cross-national differences and not on attitude change over time, I combined data from different time periods (from 1981 to 2004). Responses to all ten items consisted of 242,048 individuals in average ( $S D=$ $9,978)$ coming from 84 countries. The number of respondents within countries ranged from 417 (for the Dominican Republic) to 10,070 (for Spain) with a mean of 3,189 (SD $=1,930)$.

\section{Cultural Values}

As a measure of cultural values, I used archival data of the Schwartz Value Survey (SVS) collected by Schwartz $(1992,2006)$. The data comprise samples of both university students $(\mathrm{N}=26,024$ from 66 countries $)$ and secondary teachers $(\mathrm{N}=15,975$ from 55 countries) and was collected in a similar timeframe as the EVS/WVS (from 1988 to 2002). Further information on the samples is available in Schwartz (2006). Respondents rated the importance of each of 58 values as a guiding principle in their life on a 9-point scale ranging from -1 (opposed to my values), to 7 (of supreme importance). I used those 45 items that, according to Schwartz (2006), are crossculturally equivalent in their meaning. By analyzing the value items at the country-level with smallest space analysis (a form of multi-dimensional scaling), Schwartz derived 
Chapter four: Predicting moral attitudes with cultural values seven distinct value orientations which are arranged in a circular order: Affective and Intellectual Autonomy, Egalitarianism, Harmony, Embeddedness, Hierarchy and Mastery. Adjacent value orientations signify value compatibilities, whereas opposite ones imply value conflicts and can be summarized in bipolar value dimensions. I derived country scores for the value orientations by aggregating the data at the countrylevel and averaging the items that fall into the respective value domain as reported by Schwartz (2006). To reduce the likelihood of multicollinearity in the regression model, I decided to average the two conceptually similar value orientations Affective and Intellectual Autonomy (see also Schwartz, 2006). Since, I was interested in broad cultural dimensions I used a single country score for each of the three bipolar value orientations, i.e. Autonomy (positive pole) versus Embeddedness (negative pole), Egalitarianism (positive pole) versus Hierarchy (negative pole) and Harmony (positive pole) versus Mastery (negative pole) (cf. Schwartz, 2006).

\section{Linking EVS/WVS Data with SVS Data}

In order to conduct a multi-level regression analysis, I needed to link the individual-level data (level 1) on moral attitudes from the EVS/ WVS to the Schwartz' country-level data (level 2) on cultural values. The variable that linked the two datasets was 'country'. For instance, the responses of individuals from Brazil on their moral attitudes (EVS/ WVS data) were linked to the aggregated country-level responses on values also obtained from individuals from Brazil. Missing values were deleted listwise within level 1 and level 2 as well as across levels (e.g. if data were available for a country at level 2, but not at level 1 and vice versa). Hence, after matching the datasets, I obtained a somewhat reduced dataset. Furthermore, I first analyzed the effect of cultural values on individuals' moral attitudes with SVS student scores. I then validated the effect with the teacher scores. Hence, I obtained the following two datasets: (1) data 
from 119,743 individuals at level 1 and from 51 countries at level 2 based on aggregated students' value responses, (2) data from 100,621 individuals at level 1 and from 43 countries at level 2 based on aggregated teachers' value responses. Note, that the level 1 datasets overlap and only level 2 data are entirely different (aggregated student scores versus teacher scores).

\section{'Control Variables'}

Economic Development of Countries. As a measure of socio-economic status of countries, I utilized the Human Development Index (HDI) from the United Nations Development Programme (2006). It is composed of a number of important aspects characterizing the development of countries, which are: life expectancy at birth, adult literacy rate, index for primary, secondary and tertiary education, and GDP per capita (PPP). I replaced the missing value for the HDI index for Taiwan with the Hong Kong score which shows relatively similar level of economic development (see, for example, the CIA World Factbook ${ }^{13}$ ). I computed an average HDI score for each country across the time span 1981 and 2004. This was done to match the HDI scores to the timeframe of data collected for the EVS/ WVS and SVS.

Individual-level Predictors. I included individuals' socio-demographic variables in the model to examine whether they accounted for differences in moral attitudes. Guided by previous research (Harding \& Phillips, 1986), I selected the variables age (labelled as X003 in the EVS/ WVS dataset), gender $(X 001,1=$ male, $2=$ female), religiosity $($ F063, $1=$ God not at all important, $10=$ God very important $)$, political orientation $(E 033,1=$ left-wing oriented, 10 = right-wing oriented) and income $(X 047$, $1=$ lower step, $10=$ tenth step $^{14}$ ).

\footnotetext{
${ }^{13}$ Retrieved, June 2009, from https://www.cia.gov/index.html

${ }^{14}$ Respondents were presented with a ten-step scale of incomes and asked to choose the group their household belongs to before taxes and other deductions.
} 


\section{Analytical Strategy}

\section{Construct Equivalence of Moral Attitudes}

Prior to conducting the regression analysis, I needed to ensure that individuals from different countries differentiate between dishonest, illegal and personal-sexual issues. If the issues had different meanings across cultures, I would be comparing apples with oranges in the subsequent analytical models. A way to establish equivalence in meaning of items is to examine whether the scale in question has the same structure across cultures. This is called structural or construct equivalence (van de Vijver \& Leung, 1997). Hence, the first analysis targeted the factor structure of the scale and its cross-cultural application. The resulting factors were then treated as dependent variables in the subsequent multilevel regression analyses.

Construct equivalence can be examined with confirmatory factor analysis (CFA) which allows testing a priori specified hypotheses regarding the underlying structure of a scale (van de Vijver \& Leung, 1997). Past research has shown that six out of the ten attitudinal items formed a subscale labeled as personal-sexual attitudes. However, the underlying meaning of the four remaining items is inconclusive to date (cf. Harding \& Phillips, 1986). As it was not possible to derive a clear hypothesis on the factor structure of all items, I first explored it with principal component analysis (PCA) and then evaluated it with confirmatory factor analysis (CFA). Since exploratory and confirmatory analyses should be conducted on independent samples, I randomly split the total dataset into two equal datasets and performed the exploratory analysis on one dataset and the confirmatory analysis on the other. In order to avoid conducting an exploratory factor analysis for each country separately, which would have resulted in 84 PCA's, I performed a factor analysis on the overall pooled within-country correlation matrix. This procedure weighs the correlation matrices of each country equally, so that 
they have the same influence on the overall matrix (Fontaine \& Fischer, in press; Muthén, 1994). The PCA represents the average factor solution at the individual-level across all cultural groups. I then used the second dataset and tested the fit of the factor solution with CFA. I first examined whether the factor structure fits well for the pooled within-country data. If so, I proceeded using it as a norm solution for a multi-group CFA in which I also tested whether the average structure fits well in each country individually. This is the first study to report such multi-group analyses for the MDBS across the complete WVS data set.

\section{Multilevel Regression Analysis}

I used HLM 6 for the multilevel analyses, since individuals are nested within countries. Multilevel models have the advantage that they simultaneously analyze variables from different levels and that they statistically take into account the various dependencies occurring in hierarchical datasets (Raudenbush \& Bryk, 2002). In HLM terms, individuals' moral attitudes and their socio-demographic characteristics were the level-1 variables. Cultural values and socio-economic development of countries were the level-2 variables. HLM can compute regressions within and across countries. The analytical procedure consisted here in five steps. First, I conducted a one-way ANOVA (Model 1) in which no predictors were included (all following equations are adopted from Raudenbush \& Bryk, 2002):

$$
Y_{\mathrm{ij}}=\gamma_{00}+u_{0 j}+r_{i j}
$$

$Y_{\mathrm{ij}}$ is the dependent variable (DV), $i$ indexes the individual within a country and $j$ indexes the country; $\gamma_{00}$ is the grand mean of the DV across all countries when all predictors are zero, $u_{0 j}$ is the unique effect of country $j$ on the intercept and $r_{i j}$ is the deviation for case $i$ from its country $j$. This model allowed me to examine whether there was sufficient variation in mean differences in moral attitudes within and between 
countries. Using the Intraclass correlation coefficient (ICC), I determined how much of the total variance was associated with country differences as opposed to individual differences:

$$
I C C=\tau_{00} /\left(\tau_{00}+\sigma^{2}\right)
$$

in which $\tau_{00}$ is the variance between countries and $\sigma^{2}$ is the level-1 variance.

Secondly, if there was sufficient variation at both levels, I tried to account for it by first examining socio-demographic variables as predictors (Model 2). In this model the slope coefficients of the individual-level predictors were set to be fixed across countries and only the intercept was allowed to vary randomly:

$$
Y_{\mathrm{ij}}=\gamma_{00}+\gamma_{10} * X_{\mathrm{ij}}+u_{0 j}+r_{i j}
$$

in which $\gamma_{10} * X_{\mathrm{ij}}$ is a level-2 regression coefficient times a level-1 predictor. The percentage of explained variance was computed with the following equation:

$$
R^{2}=\left(\sigma_{\text {ANOVA }}^{2}-\sigma_{\text {Model }}^{2}\right) / \sigma_{\text {ANOVA }}^{2}
$$

Third, I allowed the slopes to vary randomly across countries (Model 3). This permitted me to test for cross-cultural variability in regression coefficients and to assess whether a random slope model fitted the data better than a fixed slope model:

$$
Y_{\mathrm{ij}}=\gamma_{00}+\gamma_{10} * X_{\mathrm{ij}}+u_{0 j}+u_{1 j} * X_{\mathrm{ij}}+r_{i j}
$$

in which $u_{1 j} * X_{\mathrm{ij}}$ is the deviation of the country slopes from the overall slope for the level-1 predictor $X_{\mathrm{ij}}$.

Fourth, I added country-level predictors to the best fitting level-1 model and examined whether they accounted for cross-cultural differences in moral attitudes (i.e. the intercept $\gamma_{00}$ ) when individual differences were controlled for (Model 4). Cultural values as country-level predictors were first derived from students' responses in Schwartz' dataset: 
$Y_{\mathrm{ij}}=\gamma_{00}+\gamma_{01} * W_{\mathrm{j}}+\gamma_{10} * X_{\mathrm{ij}}+u_{0 j}+u_{l j} * X_{\mathrm{ij}}+r_{i j}$

in which $\gamma_{01} * W_{\mathrm{j}}$ stands for a level-2 regression coefficient times a level-2 predictor. The percentage of explained variance for country-level predictors was computed with the following equation:

$$
R^{2}=\left(\tau_{00 \text { Anova }}-\tau_{00 \text { Model }}\right) / \tau_{00 \text { Anova }}
$$

Fifth, if the slopes in Model 3 proved to be significantly different across countries, I entered cross-level interaction terms $\left(\gamma_{11} * W_{\mathrm{j}} * X_{\mathrm{ij}}\right)$ to examine whether they may explain the significant slope variations (Model 5):

$$
Y_{\mathrm{ij}}=\gamma_{00}+\gamma_{01} * W_{\mathrm{j}}+\gamma_{10} * X_{\mathrm{ij}}+\gamma_{11} * W_{\mathrm{j}} * X_{\mathrm{ij}}+u_{0 j}+u_{1 j} * X_{\mathrm{ij}}+r_{i j}
$$

The final model was validated with cultural values derived from teachers' responses from Schwartz' dataset.

Throughout the five models, I excluded non-significant predictors in subsequent models. I applied grand-mean centring to all predictor variables except for gender which was dummy coded $(1=$ female). I used full maximum likelihood (ML) as a method of estimation instead of restricted maximum likelihood (REML), since it has the practical advantage that any pair of nested models can be tested for significant differences with a likelihood ratio test (see Tabachnick \& Fidell, 2007, p. 830):

$$
\chi^{2}=(\text { Deviance })_{\text {simpler model }}-(\text { Deviance })_{\text {complex model }}
$$

Degree of freedom for this chi-square test is the difference in the number of parameters for the models that are compared.

\section{Results}

\section{Factor Structure of the MDBS}

The PCA for the pooled within-country data in the first random split-half sample yielded a clear two-factor solution based on the scree plot and Kaiser's criterion. Factor one consisted of six items and replicated the personal-sexual component (eigenvalue 
3.50). Factor two comprised the remaining four items which were labeled as attitudes towards 'dishonest-illegal' behaviours (eigenvalue 1.61). Table 4.1 shows the factor loadings. The two factors explained together $51.1 \%$ of the total variance.

Table 4.1: PCA factor loadings of the MDBS derived from the pooled sample.

\begin{tabular}{llcc}
\hline & & \multicolumn{2}{c}{ Factor Loadings } \\
\cline { 3 - 4 } Morally Debatable Behaviour Items & $\begin{array}{c}\text { Attitudes towards } \\
\text { Personal-sexual } \\
\text { Behaviours } \\
\text { (Factor 1) }\end{array}$ & $\begin{array}{c}\text { Attitudes towards } \\
\text { Dishonest-illegal } \\
\text { Behaviours } \\
\text { (Factor 2) }\end{array}$ \\
\hline 1. Someone accepting a bribe & .17 & $\mathbf{. 6 4}$ \\
2. Claiming government benefits & .03 &. $\mathbf{7 0}$ \\
3. Avoiding a fare on public transport & .14 &. $\mathbf{7 4}$ \\
4. Cheating on taxes & .15 &. $\mathbf{7 5}$ \\
5. Suicide & & .21 \\
6. Euthanasia & .60 & .11 \\
7. Prostitution & .64 & .22 \\
8. Homosexuality & .69 & .10 \\
9. Divorce & .71 & .05 \\
10. Abortion & .75 & .06 \\
\hline & & .77 & $16.08 \%$ \\
& Explained variance & & $(1.61)$ \\
\hline
\end{tabular}

Note. Employed rotation method was varimax with Kaiser Normalization. Substantial factor loadings on Factor 1 and Factor 2 are in bold.

I conducted a CFA (in AMOS 6) in the second split-half sample to test whether a two-factor model fitted the pooled-within data better than a one-factor model, using the pooled-within covariance matrix as data input. Each item was specified as loading on one single latent factor. The latent constructs in the two-factor model were assumed to be intercorrelated. In none of the models errors were allowed to correlate. The hypothesized two-factor model yielded a significant chi-square statistic $\left(\chi^{2}(34)=\right.$ 15616.54, $p<.001$ ) which is common with big sample sizes (Bentler, 1992b). Yet, other goodness of fit indices suggested that the two-factor model fitted the data reasonably well. Bentler (1992b) suggested a cutoff value beyond .90 for approximate 
fit indices such as the Normed Fit Index (NFI) or the Comparative Fit Index (CFI). The fit indices were all within this range: The NFI was .93 and CFI was .93. The root-meansquare error of approximation (RMSEA) which considers the parsimony of the model was .07 , which is below the .08 cutoff value that indicates approximate fit (Browne $\&$ Cudeck, 1992). The factor loadings were all significant and sizeable for the dishonestillegal factor (.53-.67) and the personal-sexual factor (.55-.69). There was a correlation of moderate size between the two factors $(r=.44, p<.001)$. A comparison with the one-factor model showed that it yielded worse fit indices $(\mathrm{NFI}=.75 ; \mathrm{CFI}=.75$; and $\operatorname{RMSEA}=.13)$, and also a significant chi-square statistic $\left(\chi^{2}(35)=52981.64, p<.001\right)$. Based on the goodness of fit indices for both models, I concluded that the two-factor structure fitted the data well in the pooled-within sample and I used it as a norm solution for the subsequent multi-group analyses.

I next tested configural invariance by examining the cross-cultural validity of the hypothesized two-factor structure across all 84 countries. Hence, I tested whether the two-factor structure fitted the data of all countries when no cross-country constraints are imposed. The results showed again a significant chi-square $\left(\chi^{2}(2822)=136425.26, p<\right.$ $.001)$. The values for NFI, CFI and RMSEA (.91, .91 and .01) indicated that the twofactor structure fitted relatively well across all 84 countries. This high level is even more impressive considering that we are dealing with 84 data matrices and that these fit criteria were developed for assessing fit in single-group models. For multi-group and intercultural comparisons it may be acceptable to relax these criteria somewhat (van de Vijver \& Fischer, 2009).

I next tested for metric invariance by imposing equality constraints on the factor loadings. The results showed that the Goodness-of-fit statistics for this 84-group model were still acceptable $(\mathrm{NFI}=.91 ; \mathrm{CFI}=.91$ and $\mathrm{RMSEA}=.01)$. Cheung and Rensvold (2002) recently suggested that values for $\Delta \mathrm{CFI}$ smaller than or equal to -.01 indicate that 
Chapter four: Predicting moral attitudes with cultural values

the null hypothesis of invariance should not be rejected. In this case $\Delta$ CFI was -.006 leading me to accept invariance in factor loadings across all countries. This confirms structural equivalence for the ten items.

To summarize, I found that the ten items of the MDBS are distinguishable into six items measuring attitudes towards personal-sexual behaviour and four items measuring attitudes towards dishonest-illegal behaviour. This distinction held for both the average individual-level data as well as for each of the 84 countries. Thus, I was able to use these two factors as distinct dependent variables for the subsequent multilevel regression analyses. I computed for each individual an average score for items tapping into (1) dishonest-illegal behaviours and (2) personal-sexual behaviours, analyzing them separately in the multilevel regression models.

\section{Prediction of Moral Attitudes}

The regression results for dishonest-illegal issues are displayed in Table 4.2 and for personal-sexual issues in Table 4.3.

\section{Attitudes towards Dishonest-illegal Behaviours}

Multilevel Regression. The one-way ANOVA model (Model 1, Equation 1) yielded an estimated grand mean of $2.26(S E=0.07, p<.001)$ indicating that individuals tended to hold a rather severe attitude towards these issues (note that the rating scale ranged from 1 'never justified' to 10 'always justified'). Computing the ICC (Equation 2) showed that only about $8.7 \%$ of the total variance was associated with differences between countries. Hence, the larger part of the total variance, $91.3 \%$, was due to individual differences within countries. This partition of variance provided to some extent first evidence that the rejection of dishonest and illegal behaviours is not strongly related to culturally specific attributes. 
Table 4.2: Multilevel regression analyses for attitudes towards dishonest-illegal issues.

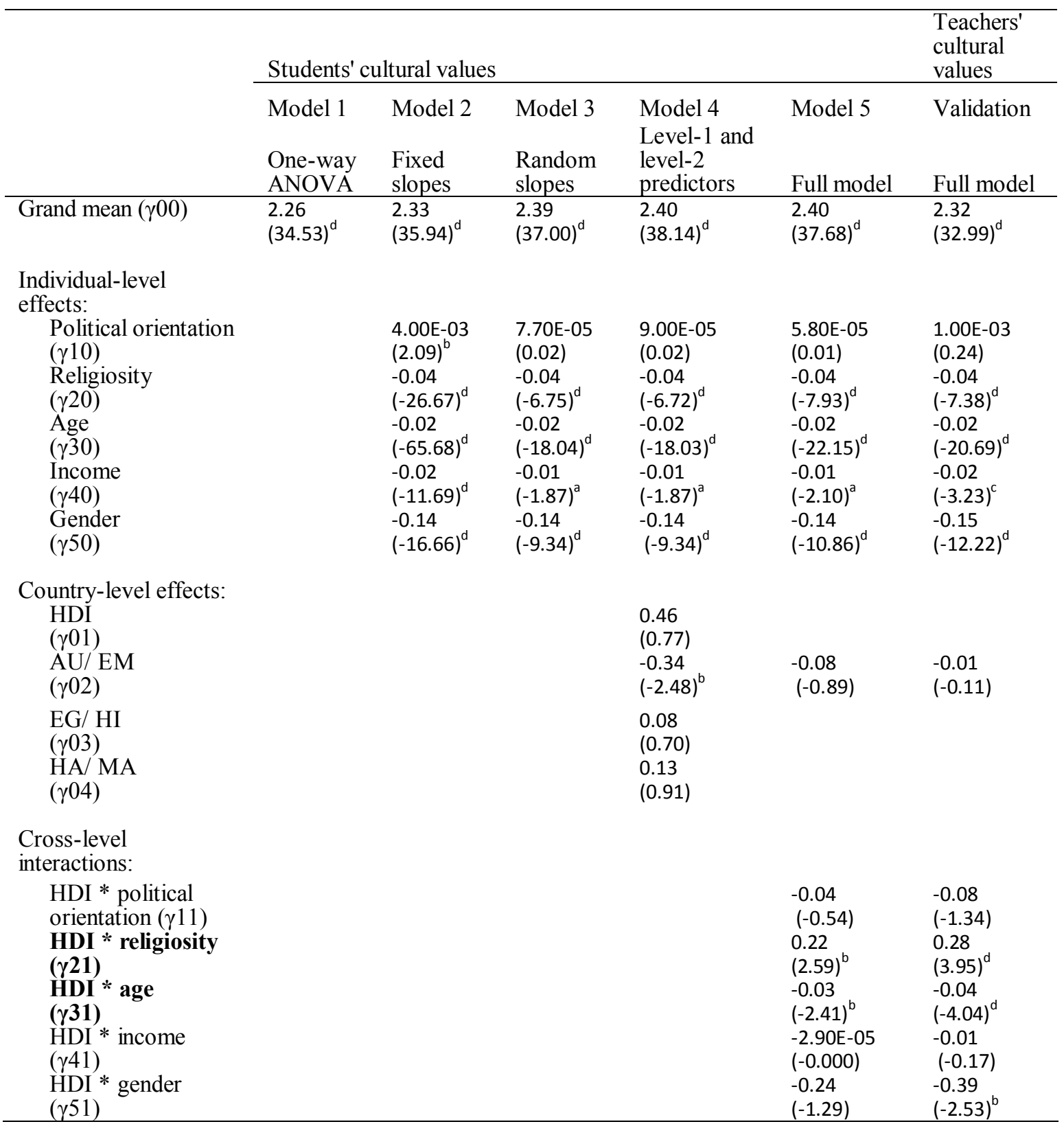




\begin{tabular}{|c|c|c|c|c|c|c|}
\hline & \multicolumn{5}{|c|}{ Students' cultural values } & \multirow{2}{*}{$\begin{array}{l}\begin{array}{l}\text { Teachers' } \\
\text { cultural } \\
\text { values }\end{array} \\
\text { Validation }\end{array}$} \\
\hline & $\begin{array}{l}\text { Model } 1 \\
\text { One-way } \\
\text { ANOVA }\end{array}$ & $\begin{array}{l}\text { Model } 2 \\
\text { Fixed } \\
\text { slopes }\end{array}$ & $\begin{array}{l}\text { Model } 3 \\
\text { Random } \\
\text { slopes }\end{array}$ & $\begin{array}{l}\text { Model } 4 \\
\text { Level-1 and } \\
\text { level-2 } \\
\text { predictors }\end{array}$ & Full model & \\
\hline \multicolumn{7}{|l|}{$\begin{array}{l}\text { Cross-level } \\
\text { interactions: }\end{array}$} \\
\hline $\begin{array}{l}\text { AU/ EM }{ }^{*} \text { political } \\
\text { orientation }(\gamma 12)\end{array}$ & & & & & $\begin{array}{l}-0.01 \\
(-0.93)\end{array}$ & $\begin{array}{l}-8.00 \mathrm{E}-03 \\
(-0.85)\end{array}$ \\
\hline $\begin{array}{l}\mathrm{AU} / \mathrm{EM}^{*} \\
\text { religiosity }(\gamma 22)\end{array}$ & & & & & $\begin{array}{l}8.15 \mathrm{E}-04 \\
(0.05)\end{array}$ & $\begin{array}{l}-0.01 \\
(-0.95)\end{array}$ \\
\hline $\begin{array}{l}\mathrm{AU} / \mathrm{EM}^{*} \text { age } \\
(\gamma 32)\end{array}$ & & & & & $\begin{array}{l}-3.00 E-03 \\
(-1.35)\end{array}$ & $\begin{array}{l}-1.00 \mathrm{E}-03 \\
(-0.67)\end{array}$ \\
\hline $\begin{array}{l}\mathrm{AU} / \mathrm{EM}^{*} \text { income } \\
(\gamma 42)\end{array}$ & & & & & $\begin{array}{l}1.00 \mathrm{E}-03 \\
(0.06)\end{array}$ & $\begin{array}{l}2.00 \mathrm{E}-03 \\
(0.152)\end{array}$ \\
\hline $\begin{array}{l}\text { AU/ EM }{ }^{*} \text { gender } \\
(\gamma 52)\end{array}$ & & & & & $\begin{array}{l}-0.05 \\
(-1.37)\end{array}$ & $\begin{array}{l}-0.02 \\
(-0.70)\end{array}$ \\
\hline $\begin{array}{l}\text { EG/ HI }{ }^{*} \text { political } \\
\text { orientation }(\gamma 13)\end{array}$ & & & & & $\begin{array}{l}8.00 \mathrm{E}-03 \\
(0.75)\end{array}$ & $\begin{array}{l}-4.00 \mathrm{E}-03 \\
(-0.38)\end{array}$ \\
\hline $\begin{array}{l}\text { EG/ HI }{ }^{*} \text { religiosity } \\
(\gamma 23)\end{array}$ & & & & & $\begin{array}{l}-0.04 \\
(-3.20)^{c}\end{array}$ & $\begin{array}{l}-0.04 \\
(-3.17)^{c}\end{array}$ \\
\hline $\begin{array}{l}\mathrm{EG} / \mathrm{HI}^{*} \text { age } \\
(\gamma 33)\end{array}$ & & & & & $\begin{array}{l}2.00 \mathrm{E}-03 \\
(1.44)\end{array}$ & $\begin{array}{l}2.00 \mathrm{E}-03 \\
(0.89)\end{array}$ \\
\hline $\begin{array}{l}\mathrm{EG} / \mathrm{HI}{ }^{*} \text { income } \\
(\gamma 43)\end{array}$ & & & & & $\begin{array}{l}-0.04 \\
(-2.97)^{c}\end{array}$ & $\begin{array}{l}-0.040 \\
(-3.31)^{c}\end{array}$ \\
\hline $\begin{array}{l}\mathrm{EG} / \mathrm{HI}^{*} \text { gender } \\
(\gamma 53)\end{array}$ & & & & & $\begin{array}{l}-6.00 \mathrm{E}-03 \\
(-0.20)\end{array}$ & $\begin{array}{l}0.02 \\
(0.51)\end{array}$ \\
\hline $\begin{array}{l}\mathrm{HA} / \mathrm{MA}^{*} \text { political } \\
\text { orientation }(\gamma 14)\end{array}$ & & & & & $\begin{array}{l}1.78 \mathrm{E}-04 \\
(0.01)\end{array}$ & $\begin{array}{l}0.02 \\
(1.29)\end{array}$ \\
\hline $\begin{array}{l}\mathrm{HA} / \mathrm{MA}^{*} \\
\text { religiosity }(\gamma 24)\end{array}$ & & & & & $\begin{array}{l}0.03 \\
(1.69)^{a}\end{array}$ & $\begin{array}{l}0.03 \\
(2.22)^{b}\end{array}$ \\
\hline $\begin{array}{l}\mathrm{HA} / \mathrm{MA}^{*} \text { age } \\
(\gamma 34)\end{array}$ & & & & & $\begin{array}{l}-4.44 \mathrm{E}-04 \\
(-0.23)\end{array}$ & $\begin{array}{l}-8.16 \mathrm{E}-04 \\
(-0.43)\end{array}$ \\
\hline $\begin{array}{l}\text { HA/ MA }{ }^{*} \text { income } \\
(\gamma 44)\end{array}$ & & & & & $\begin{array}{l}0.05 \\
(3.20)^{c}\end{array}$ & $\begin{array}{l}0.05 \\
(3.96)^{d}\end{array}$ \\
\hline $\begin{array}{l}\text { HA/ MA }{ }^{*} \text { gender } \\
(\gamma 54)\end{array}$ & & & & & $\begin{array}{l}0.01 \\
(0.39)\end{array}$ & $\begin{array}{l}-4.23 \mathrm{E}-04 \\
(-0.01)\end{array}$ \\
\hline \multicolumn{7}{|l|}{ Explained variance: } \\
\hline Within countries & & $4.9 \%$ & $6.3 \%$ & $6.3 \%$ & $6.3 \%$ & $6.5 \%$ \\
\hline Between countries & & & & $9.6 \%$ & $7.1 \%$ & $4.1 \%$ \\
\hline
\end{tabular}

${ }^{\mathrm{a}} p<.10 ;{ }^{\mathrm{b}} p<.05 ;{ }^{\mathrm{c}} p<.01 ;{ }^{\mathrm{d}} p<.001$.

Note. $\mathrm{AU} / \mathrm{EM}=$ Autonomy vs. Embeddedness, EG/ HI = Egalitarianism vs. Hierarchy, HA/ MA = Harmony vs. Mastery, HDI = Human Development Index (UNDP, 2006). Entries are unstandardized regression coefficients with T-ratios in parentheses. All predictors are grand-mean centred, except for gender which is dummy coded $(1=$ female). Coefficients in bold are highly significant moderations at $p<$ .01 for both the student-based and teacher-based model. 
However, there was some variability between-countries and a chi-square test indicated that this was significantly different from zero $\left(\chi^{2}(50)=28157.90, p<.001\right)$. I proceeded testing whether country-level predictors accounted for the between-country variance when individual-level predictors were controlled for.

The second model included only individual-level predictors with fixed slopes (Equation 3). Consistent with past research (Harding \& Phillips, 1986), I found that females were stricter in their moral judgments and that age, religiosity, and right-wing orientation were also related to a stricter moral outlook. Income was a significant negative predictor: lower income was related to a more tolerant attitude towards dishonest-illegal behaviours. This individual-level model explained $4.9 \%$ of the withincountry variance (Equation 4).

The next model (Model 3, Equation 5), in which I tested whether slopes for individual-level predictors vary across countries showed that there was indeed considerable variation across all variables (political orientation: $\chi^{2}(50)=219.60, p<$ .001 , religiosity: $\chi^{2}(50)=709.70, p<.001$, gender: $\chi^{2}(50)=144.68, p<.001$, age: $\chi^{2}(50)=495.84, p<.001$, and income: $\left.\chi^{2}(50)=614.89, p<.001\right)$. Although the predictor variable 'political orientation' showed varying slopes across countries, its overall regression coefficient for the prediction of moral attitude did not reach statistical significance anymore $(p>.05)$. This indicates that political orientation is differently related to dishonest-illegal attitudes across cultures, but shows no main effect independent of cultural context. This model explained $6.3 \%$ of the within-country variance. A chi-square test for nested models (Equation 9) yielded a significant difference between the two models $\left(\chi^{2}(20)=1463.24, p<.001\right)$ leading me to conclude that the random slope model fitted the data better than the fixed slope model.

I proceeded testing whether the context variables, i.e. Schwartz' cultural values and HDI, may account for the between-country variation in dishonest-illegal issues 
when individual-level predictors are controlled for (Model 4, Equation 6). Autonomy/ Embeddedness significantly accounted for between-country differences $(B=-0.34, S E=$ $0.14, p<.05)$. However, the cultural value orientations Egalitarianism/ Hierarchy and Harmony/ Mastery as well as HDI were not significant predictors. This model explained $9.6 \%$ of the between-country differences (Equation 7). The finding that the cultural value orientation of Autonomy/ Embeddedness was a predictor might be attributable to a suppression effect which renders a variable statistically significant when other variables, such as level-1 predictors, are considered in the analysis (see, for example, Tabachnick \& Fidell, 2007). I tested this possibility by running a model in which only level-2 variables were included. I found that Autonomy/ Embeddedness was not a significant predictor anymore $(B=-0.31, S E=0.17, p>.05)$. Egalitarianism/ Hierarchy, Harmony/ Mastery and HDI remained non-significant.

Since I found that slopes of individual-level predictors varied across countries, I ran a moderation model to explore whether cultural values and HDI accounted for these variations (Model 5, Equation 8), with all individual-level predictors and Autonomy/ Embeddedness as a country-level predictor. The findings showed that in this model Autonomy/ Embeddedness was not a significant predictor $(B=-0.08, S E=0.09, p>$ $.05)$. There were seven significant cross-level interaction effects at an alpha level of .05 (see Table 4.2). This explorative moderation model included 20 tests which increased the probability of spurious effects by $64.15 \%$. To counteract a possible Type I error, I decided to interpret only those interactions that were significant at a more stringent alpha level of .01 - for both students' and teachers' cultural values scores. I present the significant interaction findings below when discussing the teacher scores. The full regression model explained $6.3 \%$ of the within country variance and $7.1 \%$ of the between-country variance. 
Validation with Teachers' Cultural Values. I tested whether the final model could be replicated with cultural values derived from teachers' responses (see Table 4.2, right panel). Religiosity, age, and income significantly predicted stricter attitudes towards dishonest-illegal issues. Furthermore, females were stricter than males in their moral judgment and political orientation was not a predictor. The Autonomy/ Embeddedness dimension was not a significant country-level predictor. All slopes of the individual-level predictors proved to be significantly different across countries (political orientation: $\left(\chi^{2}(38)=165.63, p<.001\right)$, religiosity: $\chi^{2}(38)=412.47, p<.001$, gender: $\chi^{2}(38)=79.71, p<.001$, age: $\chi^{2}(38)=309.24, p<.001$, and income: $\chi^{2}(38)=223.74, p$ $<.001)$. There were the same moderation effects as in the previous analysis based on Schwartz' students' data and an additional significant cross-level interaction effect (i.e. gender and moral attitude moderated by HDI). In the following, I report only those interactions that were significant at $p<.01$ in both the 'student' and 'teacher' sample. There were three interactions that met this criterion, i.e. the moderation effect of (1) Egalitarianism/ Hierarchy on religiosity and moral attitude, (2) Egalitarianism/ Hierarchy on income and moral attitude, and (3) Harmony/ Mastery on income and moral attitude. These cross-level interactions are depicted in Figure 4.2 for the 'teacher' sample. The graphs show that individuals in countries emphasizing Egalitarianism are more tolerant towards dishonest and illegal issues when their religiosity and income are low compared to individuals in countries emphasizing Hierarchy. With increasing income and religiosity, however, individuals in egalitarian countries exhibit stricter moral judgments to such an extent that at a certain point they are less tolerant than individuals in Hierarchy countries. The same effect is found for Harmony/ Mastery in the sense that individuals with low income are more tolerant in Mastery countries than in Harmony countries, but with increasing income this relationship is reversed. 

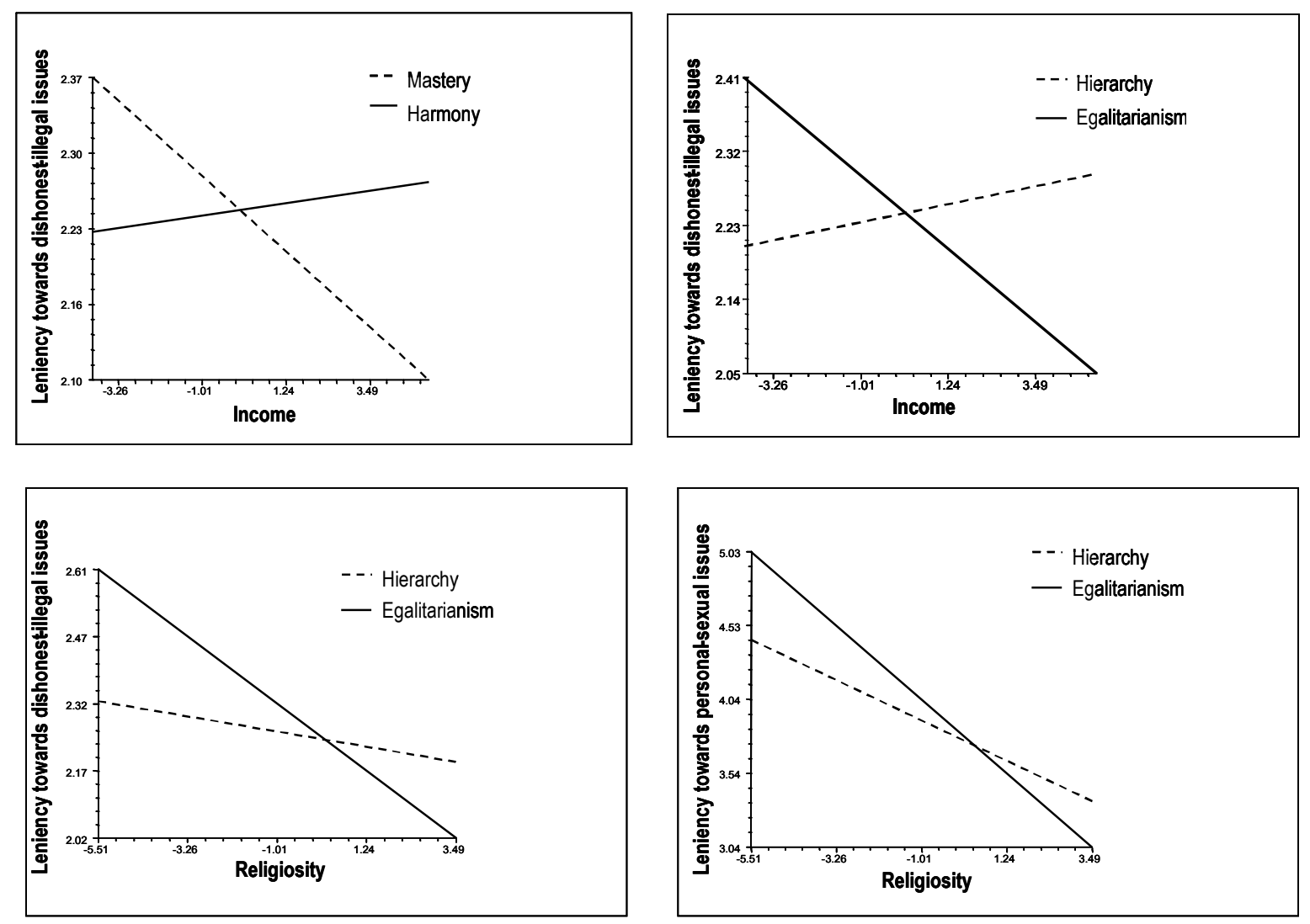

Figure 4.2: Cross-level interactions for attitudes towards dishonest-illegal and personalsexual issues based on teachers' cultural values 
Multilevel Regression. The one-way ANOVA model (Model 1, Equation 1) produced an estimated grand mean of $3.63(S E=0.14, p<.001)$. It indicated that on average individuals across countries judged these issues in a rather strict way. However, they were not as strict in their judgments as towards dishonest-illegal behaviours. The ICC (Equation 2) showed that the total variance in personal-sexual attitudes was partitioned into $21.9 \%$ between-country variance and consequently $78.1 \%$ withincountry variance. Hence, there was considerably more cross-country variance in personal-sexual issues than in dishonest-illegal ones.

Model 2 showed that right-wing orientation, religiosity, age and lower income were all significantly related to a stricter attitude towards personal-sexual issues. Contrary to previous findings (cf. Harding \& Phillips, 1986), I found that women were more tolerant than men when judging personal-sexual behaviours. The model explained $12.7 \%$ of the within-country variance (Equation 4$)$.

Model 3, which tested for random slopes across countries (Equation 5), showed that the slopes for all individual-level predictors varied significantly across countries (political orientation: $\chi^{2}(50)=1205.38, p<.001$, religiosity: $\chi^{2}(50)=1647.34, p<.001$, age: $\chi^{2}(50)=862.11, p<.001$, income: $\chi^{2}(50)=455.43, p<.001$, and gender: $\chi^{2}(50)=$ $281.75, p<.001)$. The model explained $16.1 \%$ of the within-country variance and fitted the data significantly better than the fixed slope model $\left(\chi^{2}(20)=4189.74, p<.001\right)$.

I included country-level predictors, i.e. Schwartz' cultural values and HDI, in the next model (Model 4, Equation 6). I found my hypothesis confirmed that the cultural value orientation Autonomy is related to greater tolerance towards personal-sexual behaviours. I also found support for my expectation that individuals in countries with higher level of human development tended to exhibit more lenient moral judgments. None of the other cultural value dimensions were significant predictors. In order to test 
Chapter four: Predicting moral attitudes with cultural values whether the cultural value dimension Autonomy/ Embeddedness added unique variance to the prediction of personal-sexual attitudes, I ran two additional models and statistically compared them. The first model included individual-level predictors and the two significant country-level predictors HDI and Autonomy/ Embeddedness. It explained $76.8 \%$ of the between-country variance (Equation 7 ). The second model was identical except that it contained only HDI as a country-level predictor. It explained $73.4 \%$ of the variance between countries. A chi-square test for these two nested models (Equation 9) confirmed that the regression model containing both HDI and Autonomy/ Embeddedness was significantly better than the model with HDI only $\left(\chi^{2}(1)=4.41, p<\right.$ $.05)$.

Model 5 included cross-level interactions to account for the random slopes across countries (Equation 8). Both Autonomy/ Embeddedness and HDI were still significant predictors and there were eight moderation effects ranging from marginally to highly significant (see Table 4.3). I proceeded in the same way as above for dishonest-illegal issues, to interpret only those interactions that were significant at an alpha level of .01 for the 'student' and 'teacher' scores. The findings are reported below. This final model explained $16.1 \%$ of the within country variance and $77 \%$ of the between country variance.

Validation with Teachers' Cultural Values. I obtained very similar results with cultural values derived from the teacher dataset, although the HLM dataset was reduced to somewhat less respondents and countries concerning the individual-level variables ( $\mathrm{N}$ $=100,621, \mathrm{k}=43)$. The level-1 predictors, political orientation, religiosity, age, income and gender, were all still significant. The country-level predictors HDI and Autonomy/ Embeddedness were also replicated. 
Table 4.3: Multilevel regression analyses for attitudes towards personal-sexual issues.

\begin{tabular}{|c|c|c|c|c|c|c|}
\hline & \multicolumn{5}{|c|}{ Students' cultural values } & \multirow{2}{*}{$\begin{array}{l}\text { Teachers' } \\
\text { cultural } \\
\text { values } \\
\text { Validation }\end{array}$} \\
\hline & $\begin{array}{l}\text { One-way } \\
\text { ANOVA }\end{array}$ & $\begin{array}{l}\text { Fixed } \\
\text { slopes }\end{array}$ & $\begin{array}{l}\text { Random } \\
\text { slopes }\end{array}$ & $\begin{array}{l}\text { Model } 4 \\
\text { Level-1 } \\
\text { and } \\
\text { level-2 } \\
\text { predictors }\end{array}$ & $\begin{array}{l}\text { Full } \\
\text { model }\end{array}$ & \\
\hline Grand mean $(\gamma 00)$ & $\begin{array}{l}3.63 \\
(25.07)^{d}\end{array}$ & $\begin{array}{l}3.58 \\
(31.22)^{d}\end{array}$ & $\begin{array}{l}3.61 \\
(32.60)^{d}\end{array}$ & $\begin{array}{l}3.60 \\
(32.60)^{d}\end{array}$ & $\begin{array}{l}3.60 \\
(51.38)^{d}\end{array}$ & $\begin{array}{l}3.72 \\
(45.30)^{d}\end{array}$ \\
\hline \multicolumn{7}{|l|}{ Individual-level effects: } \\
\hline $\begin{array}{l}\text { Political orientation } \\
(\gamma 10)\end{array}$ & & $\begin{array}{l}-0.06 \\
(-23.98)^{d}\end{array}$ & $\begin{array}{l}-0.06 \\
(-5.08)^{d}\end{array}$ & $\begin{array}{l}-0.06 \\
(-5.14)^{d}\end{array}$ & $\begin{array}{l}-0.06 \\
(-6.48)^{d}\end{array}$ & $\begin{array}{l}-0.06 \\
(-6.58)^{d}\end{array}$ \\
\hline $\begin{array}{l}\text { Religiosity } \\
(\gamma 20)\end{array}$ & & $\begin{array}{l}-0.19 \\
(-93.25)^{d}\end{array}$ & $\begin{array}{l}-0.17 \\
(-5.05)^{d}\end{array}$ & $\begin{array}{l}-0.17 \\
(-15.18)^{d}\end{array}$ & $\begin{array}{l}-0.17 \\
(-18.22)^{d}\end{array}$ & $\begin{array}{l}-0.17 \\
(-17.33)^{d}\end{array}$ \\
\hline $\begin{array}{l}\text { Age } \\
(\gamma 30)\end{array}$ & & $\begin{array}{l}-0.02 \\
(-52.34)^{d}\end{array}$ & $\begin{array}{l}-0.02 \\
(-2.07)^{d}\end{array}$ & $\begin{array}{l}-0.02 \\
(-2.15)^{d}\end{array}$ & $\begin{array}{l}-0.02 \\
(-14.14)^{d}\end{array}$ & $\begin{array}{l}-0.02 \\
(-14.58)^{d}\end{array}$ \\
\hline $\begin{array}{l}\text { Income } \\
(\gamma 40)\end{array}$ & & $\begin{array}{l}0.07 \\
(32.21)^{d}\end{array}$ & $\begin{array}{l}0.08 \\
(9.60)^{d}\end{array}$ & $\begin{array}{l}0.08 \\
(9.68)^{d}\end{array}$ & $\begin{array}{l}0.08 \\
(10.11)^{d}\end{array}$ & $\begin{array}{l}0.08 \\
(8.53)^{d}\end{array}$ \\
\hline $\begin{array}{l}\text { Gender } \\
(\gamma 50)\end{array}$ & & $\begin{array}{l}0.14 \\
(13.39)^{d}\end{array}$ & $\begin{array}{l}0.13 \\
(5.31)^{d}\end{array}$ & $\begin{array}{l}0.14 \\
(5.44)^{d}\end{array}$ & $\begin{array}{l}0.13 \\
(6.69)^{d}\end{array}$ & $\begin{array}{l}0.14 \\
(6.40)^{d}\end{array}$ \\
\hline \multicolumn{7}{|l|}{ Country-level effects: } \\
\hline $\begin{array}{l}\text { HDI } \\
(\gamma 01)\end{array}$ & & & & $\begin{array}{l}2.54 \\
(2.80)^{b}\end{array}$ & $\begin{array}{l}2.76 \\
(2.51)^{b}\end{array}$ & $\begin{array}{l}4.15 \\
(4.30)^{d}\end{array}$ \\
\hline $\begin{array}{l}\mathrm{AU} / \mathrm{EM} \\
(\gamma 02)\end{array}$ & & & & $\begin{array}{l}0.36 \\
(2.23)^{b}\end{array}$ & $\begin{array}{l}0.46 \\
(2.42)^{b}\end{array}$ & $\begin{array}{l}0.43 \\
(2.01)^{b}\end{array}$ \\
\hline $\begin{array}{l}\text { EG/ HI } \\
(\gamma 03)\end{array}$ & & & & $\begin{array}{l}0.05 \\
(0.36)\end{array}$ & & \\
\hline $\begin{array}{l}\mathrm{HA} / \mathrm{MA} \\
(\gamma 04)\end{array}$ & & & & $\begin{array}{l}0.11 \\
(0.64)\end{array}$ & & \\
\hline
\end{tabular}

Cross-level interactions:

HDI * political

orientation $(\gamma 11)$

$\mathrm{HDI} *$ religiosity

$(\gamma 21)$

$\mathrm{HDI} *$ age

$(\gamma 31)$

$\mathrm{HDI} *$ income

$(\gamma 41)$

HDI * gender

$(\gamma 51)$

AU/ EM * political

orientation $(\gamma 12)$

$\mathrm{AU} / \mathrm{EM} *$

religiosity

$(\gamma 22)$

AU/ EM * age

$(\gamma 32)$

\begin{tabular}{ll}
0.04 & -0.07 \\
$(0.28)$ & $(-0.52)$ \\
-0.09 & -0.02 \\
$(-0.58)$ & $(-1.21)$ \\
-0.04 & -0.04 \\
$(-1.86)^{a}$ & $(-2.44)^{b}$ \\
-0.10 & 0.13 \\
$(-0.79)$ & $(1.05)$ \\
0.270 & 0.63 \\
$(0.86)$ & $(2.19) b$ \\
-0.05 & -0.07 \\
$(-1.88)^{a}$ & $(-3.38) c$ \\
& \\
-0.02 & 0.01 \\
$(-0.85)$ & $(0.27)$ \\
$-1.00 \mathrm{E}-03$ & $-6.00 \mathrm{E}-03$ \\
$(-0.35)$ & $(-2.26) \mathrm{b}$ \\
\hline
\end{tabular}




\begin{tabular}{|c|c|c|c|c|c|c|}
\hline & \multicolumn{5}{|c|}{$\begin{array}{l}\text { Students' cultural } \\
\text { values }\end{array}$} & \multirow{2}{*}{$\begin{array}{l}\text { Teachers' } \\
\text { cultural } \\
\text { values } \\
\text { Validation }\end{array}$} \\
\hline & $\begin{array}{l}\text { Model } 1 \\
\text { One- } \\
\text { way } \\
\text { ANOV } \\
\text { A }\end{array}$ & $\begin{array}{l}\text { Model } 2 \\
\text { Fixed } \\
\text { slopes }\end{array}$ & $\begin{array}{l}\text { Model } 3 \\
\text { Random } \\
\text { slopes }\end{array}$ & $\begin{array}{l}\text { Model } 4 \\
\text { Level-1 } \\
\text { and } \\
\text { level-2 } \\
\text { predictors }\end{array}$ & $\begin{array}{l}\text { Full } \\
\text { model }\end{array}$ & \\
\hline \multicolumn{7}{|l|}{ Cross-level interactions: } \\
\hline $\begin{array}{l}\text { AU/ EM * income } \\
(\gamma 42)\end{array}$ & & & & & $\begin{array}{l}0.04 \\
(2.04)^{b}\end{array}$ & $\begin{array}{l}-0.01 \\
(-0.64)\end{array}$ \\
\hline $\begin{array}{l}\text { AU/ EM * gender } \\
(\gamma 52)\end{array}$ & & & & & $\begin{array}{l}0.11 \\
(1.92)^{a}\end{array}$ & $\begin{array}{l}0.03 \\
(0.53)\end{array}$ \\
\hline $\begin{array}{l}\mathrm{EG} / \mathrm{HI} * \text { political } \\
\text { orientation }(\gamma 13)\end{array}$ & & & & & $\begin{array}{l}-0.05 \\
(-2.47)^{b}\end{array}$ & $\begin{array}{l}-0.02 \\
(-1.01)\end{array}$ \\
\hline $\begin{array}{l}\text { EG/ HI * religiosity } \\
(\gamma 23)\end{array}$ & & & & & $\begin{array}{l}-0.06 \\
(-2.71)^{c}\end{array}$ & $\begin{array}{l}-0.08 \\
(-3.52)^{d}\end{array}$ \\
\hline $\begin{array}{l}\mathrm{EG} / \mathrm{HI} * \text { age } \\
(\gamma 33)\end{array}$ & & & & & $\begin{array}{l}2.00 \mathrm{E}-03 \\
(0.92)\end{array}$ & $\begin{array}{l}7.00 \mathrm{E}-03 \\
(2.53)^{\mathrm{b}}\end{array}$ \\
\hline $\begin{array}{l}\mathrm{EG} / \mathrm{HI} * \text { income } \\
(\gamma 43)\end{array}$ & & & & & $\begin{array}{l}-0.03 \\
(-1.55)\end{array}$ & $\begin{array}{l}-2.00 \mathrm{E}-03 \\
(-0.09)\end{array}$ \\
\hline $\begin{array}{l}\text { EG/ HI * gender } \\
(\gamma 53)\end{array}$ & & & & & $\begin{array}{l}0.02 \\
(0.40)\end{array}$ & $\begin{array}{l}0.07 \\
(1.19)\end{array}$ \\
\hline $\begin{array}{l}\mathrm{HA} / \mathrm{MA} * \text { political } \\
\text { orientation }(\gamma 14)\end{array}$ & & & & & $\begin{array}{l}0.03 \\
(1.17)\end{array}$ & $\begin{array}{l}0.02 \\
(0.99)\end{array}$ \\
\hline $\begin{array}{l}\text { HA/ MA * religiosity } \\
(\gamma 24)\end{array}$ & & & & & $\begin{array}{l}0.08 \\
(2.90)^{c}\end{array}$ & $\begin{array}{l}0.06 \\
(2.58)^{c}\end{array}$ \\
\hline $\begin{array}{l}\mathrm{HA} / \mathrm{MA} * \text { age } \\
(\gamma 34)\end{array}$ & & & & & $\begin{array}{l}-0.01 \\
(-1.72)^{a}\end{array}$ & $\begin{array}{l}-0.01 \\
(-2.69)^{c}\end{array}$ \\
\hline $\begin{array}{l}\mathrm{HA} / \mathrm{MA} * \text { income } \\
(\gamma 44)\end{array}$ & & & & & $\begin{array}{l}2.00 \mathrm{E}-03 \\
(0.10)\end{array}$ & $\begin{array}{l}-7.00 E-03 \\
(-0.34)\end{array}$ \\
\hline $\begin{array}{l}\mathrm{HA} / \mathrm{MA} * \text { gender } \\
(\gamma 54)\end{array}$ & & & & & $\begin{array}{l}-0.01 \\
(-0.17) \\
\end{array}$ & $\begin{array}{l}6.00 \mathrm{E}-03 \\
(0.10)\end{array}$ \\
\hline \multicolumn{7}{|l|}{ Explained variance: } \\
\hline Within countries & & $12.7 \%$ & $16.1 \%$ & $16.1 \%$ & $16.1 \%$ & $16.6 \%$ \\
\hline Between countries & & & & $76.7 \%$ & $77 \%$ & $77 \%$ \\
\hline
\end{tabular}

${ }^{\mathrm{a}} p<.10 ;{ }^{\mathrm{b}} p<.05 ;{ }^{\mathrm{c}} p<.01 ;{ }^{\mathrm{d}} p<.001$

Note. AU/ EM = Autonomy vs. Embeddedness, EG/ HI = Egalitarianism vs. Hierarchy, HA/ MA = Harmony vs. Mastery, HDI = Human Development Index (UNDP, 2006). Entries are unstandardized regression coefficients with T-ratios in parentheses. All predictors are grand-mean centred, except for gender which is dummy coded $(1=$ female). Coefficients in bold are highly significant moderations at $p<$ .01 for both the student-based and teacher-based model. 
All slopes of the individual-level predictors proved to be significantly different across countries (political orientation: $\left(\chi^{2}(38)=524.50, p<.001\right.$ ), religiosity: $\chi^{2}(38)=683.52$, $p<.001$, gender: $\chi^{2}(38)=158.72, p<.001$, age: $\chi^{2}(38)=401.32, p<.001$, and income: $\left.\chi^{2}(38)=392.03, p<.001\right)$. There were eight significant moderation effects which largely replicated the cross-level interactions found with the student sample (see Table 4.3). Only one of these interactions met the criterion of $p<.01$ in both the 'student' and the 'teacher' sample: Egalitarianism/ Hierarchy moderated the relationship between religiosity and attitude towards personal-sexual issues (see Figure 4.2). Individuals in egalitarian countries exhibited more lenient moral judgments compared to individuals in hierarchical countries under the condition of low religiosity. With increasing religiosity this relationship was reversed with highly religious individuals being stricter in their moral judgments in egalitarian countries than in hierarchical ones.

\section{Discussion}

The present study investigated cultural similarities and differences in individuals' attitudes towards moral issues. The aim was to shed light on the moral universalism versus relativism debate in the literature. My position is that both the universalist and cultural relativist paradigms are legitimate and contribute to our understanding of morality. The main objective of this study was to show empirically across a large number of cultural contexts, that whether morality is universal or culturally determined depends on the moral issues studied. I showed that moral attitudes can be universally differentiated into behaviours that violate the moral code of social cooperation and into behaviours that involve individual choices, based on data from the EVS and WVS across 84 countries. I conducted multilevel regression analyses which allowed me to estimate the effects of cultural values while statistically controlling for 
the effects of non-cultural variables (cf. Matsumoto \& Yoo, 2006). There are four conclusions that can be drawn based on these results.

First, consistent with evolutionary theories on the importance of cooperation for social cohesion (cf. Axelrod \& Hamilton; Broom, 2003; Gouldner, 1960; Krebs, 2008; Price, et al., 2002), I found that breaches of social cooperation, in the form of dishonest and illegal behaviours, are strongly and rather commonly disapproved. I found evidence that there is relatively little between-country variance in attitudes towards these issues. Cultural values and the socio-economic context of countries did not significantly account for the between-country variation in the full model. Hence, I conclude that attitudes towards dishonest-illegal behaviours are not strongly related to cultural values, therefore corroborating evolutionary theories on the universality of social cooperation. Maintaining the system of cooperation has widely been seen as the root of morality (cf. Durkheim, 1893/1984; Krebs \& Denton, 2005; Piaget, 1932/1965; Rawls, 1971). People are naturally inclined to behave in a socially cooperative way, since it is in everybody's interest to maintain this system and to condemn behaviours that may jeopardize it. Hence, selfishness, cheating and free-riding are recognized as undesirable behaviours and are rejected or even punished (Wilson, 1993). This is consistent with findings from qualitative research in moral psychology in which it has been shown that intentionally hitting, or stealing, without any reason are commonly judged as wrong (Turiel, 1983, 2002).

Secondly, I also believe that there is more to morality than this very basic moral code of social cooperation. I agree with cultural psychologists that a part of morality is also socially and culturally constructed (e.g. Haidt, et al., 1993; Miller, 2001; Shweder, et al., 1987). I found evidence for my hypothesis that cultural models of the self are related to moral judgments. I reasoned that because Schwartz' cultural value orientation of Autonomy emphasizes independence and self-determination, it provides a context in 
which people focus on the self and on individual rights. Hence, individuals in Autonomy-oriented countries should make more lenient judgments towards behaviours that concern their self and that are not evidently harming others. The opposite relationship applies to countries in which Embeddedness is valued, as this value orientation emphasizes duties and social obligations. My analyses showed that this hypothesis was confirmed.

I also found that part of the observed differences could be explained by a country's affluence. Economic development has been shown to influence a number of psychological variables, including value orientations (Hofstede, 1980; Schwartz, 2006), optimism (Fischer \& Chalmers, 2008), achievement motivation (Dekker \& Fischer, 2008; Maehr \& Nicholls, 1980), personality (van Hemert, van de Vijver, Poortinga, \& Georgas, 2002), subjective well-being (Diener, et al., 1995) or organizational commitment (Fischer \& Mansell, in press). However, the theoretical explanations for these consistent relationships are often more difficult to determine. This certainly requires more research to understand why affordances, associated with economic development, change moral judgments. However, it is noteworthy that values and socioeconomic development together explained almost three-quarter of the between-country variance, with the cultural value orientation Autonomy/ Embeddedness adding some incremental validity to the prediction of moral attitude.

Third, the larger part of the total variance (more than $75 \%$ ) was within countriesfor both personal-sexual and dishonest-illegal issues. I found that age, religiosity and gender were related to stricter moral judgments towards both dishonest-illegal and personal-sexual issues. Interestingly, the association between gender and moral attitude depended on the moral domain: men were more tolerant towards dishonest-illegal issues, whereas women showed more tolerance towards personal-sexual issues. Regarding dishonest-illegal issues, the result may reflect sex differences in both 
tolerance and engagement in deviant behaviour which have been found in past studies (see Kaplan \& Johnson, 2001). Concerning the personal-sexual domain, it may be that some of the items (e.g. abortion) that are specific to female issues are judged in a more lenient way by women, since more reasons are accessible to them under which this behaviour may be justifiable. A similar asymmetric pattern of association emerged with income and moral attitudes: individuals with higher income were more liberal in their attitude towards personal-sexual issues supporting past findings in which single items of the scale have been analysed (e.g. Norris \& Inglehart, 2004). Yet, for dishonest-illegal issues, I found that individuals with lower income were more tolerant. Furthermore, I found that right-wing political beliefs were only related to a stricter attitude towards personal-sexual issues. However, other individual-level predictors might be more important since the five individual-level predictors measured in the ESS/ WVS did not explain much of the within-country variance (as little as $4.9 \%$ for dishonest-illegal issues). Future research could considerably add to our understanding of these kinds of moral attitudes by identifying more 'powerful' individual-level predictors.

Fourth, the relationship between individual-level predictors and moral attitudes was not the same across countries. I tried to account for these varying slopes with the country-level variables and found four highly significant interaction effects across both student and teacher scores (see Figure 4.2). A common trend was that individuals in egalitarian countries were more tolerant towards personal-sexual and dishonest-illegal issues than individuals in hierarchical countries, when their level of religiosity and their income was low. Yet, with increasing income and religiosity they exhibited stricter moral judgments than individuals in hierarchical countries. An explanation could be that individuals in egalitarian societies are more free to develop and express lenient personal attitudes than individuals in hierarchical countries. However, religiosity seems then to compensate for the lenient cultural context. In hierarchical countries, right and wrong 
may already be prescribed by society so that there is only little scope left for religion to have much of an impact on individuals' attitudes.

The last cross-level interaction effect concerns income and dishonest-illegal issues: Individuals in Mastery-oriented countries showed more tolerance towards these behaviours than individuals in Harmony-oriented countries when their income was low. With increasing income, however, this relationship reversed. As Mastery values stand for ambition and success (Schwartz, 2006), it may be that individuals with low income regard minor rule breaking and free-riding as a legitimate way to achieve their goals or as a way to compensate for an unjust social system that is to their disadvantage. With increasing income they are more likely to be the ones who are negatively affected by behaviours that challenge the social system and their position within it (through, for example, free-riding), and hence they show less tolerance towards these behaviours. Individuals in Harmony cultures, on the contrary, try to accept others as they are, rather than to change or direct (Schwartz, 2006) which may also make them more lenient in their moral judgments and this is not much affected by their income.

\section{Limitations}

This study also has some limitations that need to be considered when evaluating the results. I obtained data from the EVS/ WVS from 1981 to 2004 (except for 19851988 which was not surveyed). Hence, individuals answered the survey during different decades which I did not take into account in the analyses. For example, I did not examine to what extent there may have been a change in attitudes within countries across different time periods. In a similar vein, I aggregated Schwartz' data on cultural values which was gathered from 1988 to 2002. However, in the case of values, Schwartz' stated that they are relatively stable across time (see Schwartz, 2006). A similar argument may be used for moral attitudes if they are regarded as influenced by 
cultural values. Since my focus in this study was on cross-national differences and not on attitude change over time, I did not take into account the time factor, but aimed at obtaining an extensive cross-national dataset by combining data across different time periods. Nevertheless, future research may look more specifically into value change over time and how it may affect moral attitudes. Another related limitation is that I analyzed the data in a cross-sectional fashion by averaging data points across different time periods. Therefore, I cannot draw conclusions on causality. Nevertheless, I have a strong theoretical argument to suggest that values as the more general concept influence the more specific concept of attitudes. Future research on moral attitudes should conduct longitudinal analyses cross-culturally to examine the causal relationships.

Another limitation is that the distinction between dishonest-illegal and personalsexual behaviours may in some cases be an artificial one. Consider the case of polygamy in Islam. Similarly to the item 'divorce', which I found to belong to the 'personal-sexual' factor, polygamy has something to do with marriage and could fall under 'personal-sexual' behaviours. Islamic countries, which tend to show an Embeddedness value orientation, should exhibit stricter judgments towards polygamy than countries emphasizing Autonomy, such as Western countries. However, we know that under Islamic law polygamy is allowed, whereas in many Western countries it is prohibited which renders it at the same time a behaviour that falls under the 'dishonestillegal' category in these countries. Hence, counter-examples may be found that contradict my results. I would like to emphasize at this point that the findings must be understood in the context of the items that have been assessed. Nevertheless, in regard to these items I am confident that they are cross-culturally comparable which I demonstrated with the test of equivalence.

Eventually, I would like to point out that I did not use all items of the MDBS as they were not constantly employed across all time waves and countries in the EVS/ 
WVS. It may be interesting to examine whether, besides the issues of fairness and matters of life and death, there are also other moral domains. The scale contains, for example, items on environmental behaviour and medical ethics such as experiments with embryos. Future research may examine whether attitudes towards these issues are comparable (or equivalent respectively) across a selection of countries and whether there are any cross-cultural similarities or differences.

\section{Conclusion}

The present study has demonstrated that individualistic and collectivistic values do predict moral attitudes in the domain of individual choices of life and death issues. While cross-cultural theorists have criticised that the value orientation individualism/ collectivism (or Autonomy/ Embeddedness respectively) is a 'catchall' explanation for any observed differences (e.g. Brewer \& Chen, 2007; Kağıtçıbaşı, 1997; Poortinga, 2003), I demonstrated that this dimension has its limits. Here I provided some evidence for its discriminant validity, i.e. it does not relate strongly to moral issues concerning dishonest and illegal behaviours. I also have shed light on the moral universalismrelativism debate by separating morality into the issues of fairness/ social cooperation, which I found to be judged in a nearly universal way, and the concept of individual rights which I found to be influenced by cultural models of the self. Hence, I showed that there are both universal and idiosyncratic aspects of morality across cultures. By adopting a multilevel approach, in which I considered both contextual and individual variation in moral attitudes, I believe that I contributed to research investigating important social and moral issues in a global context. 


\section{CHAPTER FIVE: STUDY 3}

\section{Identifying Cultural Differences and Similarities through the Explicit Measurement of Moral Values}

\section{Introduction}

Values are widely seen as one of the core aspects of culture (e.g. Triandis, 2001; Schwartz, 1994, 2006). Cultural value priorities can be investigated by examining the value hierarchies of cultural groups. A culture' s value hierarchy reflects the normative preference of certain life principles (Wan, Chiu, Peng, et al., 2007). Cultural value hierarchies have typically been measured by asking individuals about their personal values (self-importance ratings) and statistically aggregating the responses at the culture-level (see Schwartz, 1994). In chapter two, I argued that deriving cultural values from self-importance ratings leads to important conceptual and methodological issues. There are dynamic negotiations among individuals who share a culture, which is not reflected in the statistical aggregation of personal values (cf. Wan, Chiu, Tam, et al., 2007). There is in particular the issue that the actual self-importance of values does not take into account the inherently social function of cultural values. An important social function of culture is to guide members of a group in their behaviour and aspirations, so that collective life is organized and operates smoothly. From this perspective, culture draws boundaries on what is accepted, appropriate and socially desirable. Culture can then be regarded as inherently related to what is regarded as right or wrong or to a 'prescriptive' morality which stipulates what one should do. 
Theorists in moral psychology have already pointed out that culture and morality share an intricate and intimate relationship and that it is even difficult to delineate where culture ends and morality begins (e.g. Eckensberger, 2007; Miller, 2001). Hence, knowing what kind of moral values are prioritized in different cultures can provide an important insight into cultural differences.

The approach taken here regards culture as a product of collective processes rather than a mere aggregate of individuals' personal characteristics (see also Wan, et al., 2007). The critique on previous operationalizations of cultural values is not targeted at the statistical aggregation of group data as such, as this is a necessary procedure to derive macro-level data (cf. Liska, 1990) and will also be employed in the present study. Rather, the critique is targeted at what exactly is aggregated to derive cultural variables. The point has been made in chapter two that measuring values as personal moral values or societal expectations captures what is prescriptive in a certain culture and is therefore closer to the definition of culture as exerting a "guidance function" (Baldwin, et al., 2006, p. 38) to organize collective life (Kluckhohn \& Strodtbeck, 1961). Nevertheless, Study 2 in this thesis has demonstrated that cultural values based on personal importance ratings performed well in the prediction of individuals' moral attitudes. Any new proposed measures of cultural values must then perform equally well within this research context or add incremental validity over and above aggregated importance ratings. The present study is targeted at answering this question by examining which values of the Schwartz Value Survey (SVS, Schwartz, 1992) are personal moral values and societal expectations across different cultures.

Furthermore, the aim in this study is to examine values as predictors at the individual- and the culture-level. A direct comparison of values at both levels of analysis requires using the same value types, i.e., it involves equating the individualand culture-level value types. This practice has commonly been considered as a fallacy 
(e.g., Hofstede, 1980; Schwartz, 1994). Nevertheless, there are a number of cases in which Schwartz' individual-level value types have been used to describe cultures theoretically (Kağıtçıbaşı, 1997) and empirically (Lönnqvist, et al., 2009; Verkasalo, et al., 2009). Since it is not appropriate to apply culture-level constructs to individuals (Hofstede, 1980), I will follow previous research and apply individual-level constructs at the culture-level. This approach will allow me to investigate the unique contribution of values at the culture-level after controlling for values at the individual-level in the prediction of the dependent variable.

In the course of exploring and analyzing new value measures assessing prescriptive beliefs at the individual- and culture-level, a number of additional questions are answered such as: To what extent do the different value measures yield indeed convergent or divergent results? Do the proposed value measures capture cultural differences consistent with the most prominent cultural distinctions, i.e. the individualism/ collectivism dimension? To the best of my knowledge, this study is the first to assess which values from the SVS are regarded as prescriptive in different cultures.

\section{An Alternative Value Measure}

Chapter two provided an outline of how values may be measured by capturing their prescriptive aspect. It was suggested that there are two classes of prescriptive values: (1) those that have been internalized and are highly relevant to the individuals' self-concept, called 'personal-moral' values, and (2) those that are perceived as societal expectations. These values may be measured through the SVS by simply adapting the response scale to the respective content. Hence, societal expectations may be measured by asking individuals to what extent they believe they are expected to strive for the respective value in their society versus to what extent it is their free choice to strive for 
it. A similar response format and content has already been proposed for the measurement of social norms (cf. Aijzen, 1991; Kristiansen \& Hotte, 1996; Schwartz \& Tessler, 1972). Societal expectation ratings should elicit cultural variation since they assess what is prescriptive in a given society, which varies from society to society (cf. Haidt, 2001; Shweder, et al., 1997). In Kohlberg's (1984) terms, societal expectations would belong to the social conventional domain, however, consistent with cultural theories in moral psychology (Haidt, 2001; Miller, et al., 1990; Shweder, et al., 1997), social conventions are here regarded as part of the moral domain.

In the case of personal moral values, individuals can be asked to rate each value on a response scale indicating to what extent they believe the value is a moral value. This item possesses face validity as an assessment of personal moral values. However, since the term 'moral' is somewhat abstract, ambiguous even among experts (see introduction of this thesis) and may be understood differently depending on the layperson's conception of morality, further response scales tapping into the realm of moral values are worthwhile to explore. Another possible response scale related to morality is the question of how right or wrong it is to strive for a certain value. The terms right and wrong are strongly associated with the concept of morality. In the literature, they are used in an interchangeable fashion (cf. Wierzbicka, 2007). Hence, if individuals are asked about which values are right or wrong, their responses should be a reflection of their moral beliefs. Nevertheless, cross-cultural linguists have also pointed out the difficulty of translating the English meaning of right and wrong into other languages. Wierzbicka (2006), for instance, suggested that right and wrong possesses a unique meaning in the English language. She found that only the evaluation of good and bad was universal in cross-cultural studies. It seems that in all languages and cultures people distinguish between 'doing something bad' and 'doing something good' (for more details, see Wierzbicka, 2001). Because of the controversy whether the widely 
established term right and wrong in the Anglophone literature on morality would hold the same meaning for laypeople in different cultures, an additional response scale defined by the poles good and bad is worthwhile to look at when assessing personal moral values.

\section{Which Values May Be Moral Values? Examining Value Hierarchies}

Group-based value hierarchies are particularly interesting for cross-cultural research, because they indicate the normative preferences of life principles in the respective cultures (Wan, Chiu, Tam, et al., 2007). A common strategy to identify the core values of a cultural group is to measure its member's personal values and to compute the sample means on the surveyed values in order to infer the value importance hierarchy of the culture (Wan, Chiu, Peng, et al., 2007). "The values that most members of the culture strongly endorse indicate what the culture's core values are" (Wan, Chiu, Peng, et al., 2007, p. 213). An intriguing question in regard to alternative value measures is whether they produce similar or different value hierarchies compared to the conventional importance ratings. Schwartz and Bardi (2001) found, for instance, that the pan-cultural value hierarchy of personal values based on students' responses from 54 nations consisted of the following rank order: benevolence, self-direction, universalism, achievement, security, conformity, hedonism, stimulation, tradition and power values. Hence, any assessment of personal values (importance ratings) should elicit a similar rank order of value types. However, the rank order may be different when personal moral values or societal expectations are assessed.

Personal moral values that rank highest may be those that have commonly be regarded as related to morality by philosophers, social scientists and laypeople alike because they are concerned with actions that affect the welfare of others (e.g. Helkama, 2004; Kurtines \& Gewirtz, 1984). Helkama (2004) recently suggested that the main 
functions of morality lie in moral problem-solving (resolving value conflicts and issues of justice), promotion of prosocial actions and control of antisocial action (resistance to temptation). He pointed out that these main functions roughly correspond to Schwartz' (1992) value types of universalism, benevolence and conformity/ tradition. Empirical research seems to corroborate the linkage between morality and the endorsement of certain value types. For instance, individuals who strongly endorse universalism and benevolence values describe themselves as more empathetic (see Helkama, 2004 for a review) which is an important individual-level variable for morality (see Eisenberg, 2000). Further empirical support comes also from a preliminary mono-cultural study conducted by Schwartz (2007b). He presented the SVS to 100 Israeli adults and asked them to indicate which of the values they consider as moral values. More than $70 \%$ of them labelled all or most of the values falling into the value types universalism, benevolence, conformity, tradition and security as moral values. Hence, when personal moral values are assessed, the highest ranking value types may be of the selftranscendence and conservation kind.

However, there may also be some cultural variation, which can be even more pronounced when values are assessed as societal expectations. Shweder et al. (1997) proposed a taxonomy called the big three of morality: the ethics of Autonomy, Community, and Divinity. Western societies tend to endorse an ethic of Autonomy with a focus on harm, rights and justice, whereas non-Western societies tend to emphasize an ethic of Community with a focus on duty, respect, obedience to authority and conformity to social role expectations (see also Haidt, et al., 1993). More recently, Haidt and colleagues refined this taxonomy by proposing the Moral Foundation Theory (Haidt \& Graham, 2006; Haidt \& Joseph, 2004) which posits that there are five sets of moral intuitions: harm/ care, fairness/ reciprocity, ingroup/ loyalty, authority/ respect, and purity/ sanctity. The first two moral foundations are referred to as the individualizing 
foundations, because the source of the moral intuitions is seen in an emphasis on the rights and welfare of individuals (Graham, et al., 2009). The latter three are referred to as the binding foundations, because the source of the moral intuitions are seen in an emphasis on group-binding loyalty, duty, and self-control. This individualizing versus binding foundation resembles strongly the individualism versus collectivism distinction in the cross-cultural literature. Hence, one would expect to find the individualism and collectivism distinction mirrored in the moral values of different cultures.

Graham et al. (2009) drew parallels between their Moral Foundation Theory and Schwartz' (1992) list of ten value types as well as Shweder et al.'s (1997) three ethics: the self-transcendence quadrant of Schwartz' multidimensional plot of values relates to the harm and fairness foundations and is also similar to the ethic of Autonomy. The adjacent conservation quadrant is related to ingroup, authority, and purity and parallels the ethic of Community and Divinity. They also indicate that Moral Foundation Theory does not offer an exhaustive taxonomy and that, for example, Schwartz' value types of achievement and hedonism may point to additional psychological mechanisms that support some moral systems. It could be that opennessto-change values, with their focus on independence and autonomy, belong to an additional moral value cluster. Hence, self-transcendence and openness-to-change values, with their general focus on fairness and justice as well as the individual's autonomy, may be very salient as societal expectations in individualistic cultures. On the other hand, conservation values, with their focus on fitting into the community and conforming to group-expectations, may be more salient in collectivistic cultures. This has not been empirically investigated before and the analysis will therefore be exploratory. If cultural differences emerged for these prescriptive values and in line with cultural theories on morality and individualism and collectivism, then they would support the utility of this value construct as a measure of the cultural context. 


\section{Cultural Values and Individuals’ Moral Attitudes}

In order to empirically test the proposed value measures as an assessment of the cultural context, I examined them in their validity as predictors of individuals' moral attitudes. Study 2 showed that cultural values accounted for individuals' attitudes towards personal-sexual behaviours. Hence, these kinds of attitudes were again examined in the present study. The items for assessing individuals' moral attitudes towards personal-sexual behaviour were derived from the Morally Debatable Behaviour Scale (MDBS, Harding \& Phillips, 1986) which has already been introduced in Study 2. The original response scale assesses the justifiability of various behaviours (e.g. abortion, divorce, prostitution) ranging from 1 ('behaviour is never justified') to 10 ('behaviour is always justified'). It may be argued that this kind of response scale measures a form of moral reasoning, defined as the "conscious mental activity that consists of transforming given information about people in order to reach a moral judgment" (Haidt, 2001, p. 818). By 'conscious', it is meant that the process is intentional, effortful and controllable and the person is aware that it is going on. Hence, if provided with an item on "abortion", for instance, and a response scale eliciting to what extent this act is justifiable, individuals may start thinking about reasons under which the behaviour may indeed be justifiable (e.g. an abortion may be seen as justifiable if it posits a health risk for the mother). Since, for almost all items there may always be reasons found under which the behaviour in question becomes justifiable, if one thinks about it long enough, the answers to the scale become rather a matter of competence in moral reasoning than an assessment of moral attitude per se. For the present study, it was considered preferable to measure moral attitudes by eliciting spontaneous moral judgments (cf. Haidt, 2001). Moral judgments may be defined as evaluations (good vs. bad) of the actions or character of a person that are made with respect to a set of values regarded as prescriptive in a culture or subculture (Haidt, 
2001). Hence, the opportunity was taken in the present study to change the original response scale from asking for the justifiability of behaviours to asking for the wrongness of the respective behaviours. Note that degrees of 'wrongness' as a response scale has already been employed in the Moral Behavior Scale (Crissman, 1942) which is a forerunner of the MDBS (see also studies by Bovasso, et al., 1991; Consalvi, 1971).

Theories regarding how cultural conceptions of the self may influence individuals' attitudes towards personal-sexual behaviours have already been discussed in chapter three. A cultural orientation towards the community (Chiu, et al., 1997; Shweder, et al., 1997) goes hand in hand with a restriction of personal choices and should be expressed in a relatively strict attitude towards personal-sexual behaviours. On the other hand a cultural orientation which focuses on the individual is accompanied by an emphasis on the individual's needs and well-being and should be expressed in a more lenient attitude towards these issues. Study 2 empirically confirmed these predictions using a multi-level model with Schwartz' cultural value dimension Autonomy vs. Embeddedness as a predictor of individuals' moral attitudes. The central research question of the present study was: Do the new measures of values predict individuals' moral attitudes in the same way as personal importance ratings of values? And do values at the culture-level still add explained variance when values at the individual-level are controlled for?

Guided by the results in Study 2, the focus is on examining values that assess a form of individualistic and collectivistic value orientation such as Autonomy and Embeddedness. Kağıtçıbaşı (1997) theoretically equated Schwartz' higher order value orientation openness-to-change versus conservation to Hofstede's (1980) culture-level dimension individualism versus collectivism, which is conceptually very similar to Schwartz' Autonomy-Embeddedness dimension (see e.g., Gouveia \& Ros, 2000). Hence, I expected that the aggregated personal importance of openness-to-change vs. 
conservation would also predict individuals' moral attitudes. A cultural emphasis on openness-to-change should be related to a more lenient moral attitude. I also expected that openness-to-change vs. conservation rated as personal moral values and societal moral values should likewise be related to individuals' moral attitudes. Moreover, these value measures should add explained variance over and above personal value ratings since they directly assess the values that are regarded as prescriptive in a certain cultural group. Furthermore, the same predictive relationship was expected for values analyzed at the individual-level; however, value ratings at the culture-level should add incremental validity to the prediction of individuals' moral attitudes.

\section{Sampling of Cultures}

Since the exploration of cultural differences and similarities was the focus of the present study, a 'systematic sampling' was aimed for (cf. van de Vijver \& Leung, 1997). Cultural groups were selected from different ends along the individualism-collectivism continuum (Hofstede, 1980; Schwartz, 1994). This bipolar value orientation is the most important of all cultural dimensions and has been widely used for comparing human values across cultures (see e.g. Gouveia \& Ros, 2000; Kağıtçıbaşı, 1997; Triandis, 1995; Triandis, 2001). The reason for using the individualism-collectivism dimension as a sampling criterium was as follows: If the sampled cultural groups showed the expected similarities and differences in the proposed measures of values according to the individualism-collectivism distinction, it would indicate that the new measures converged with one of the most prominent distinctions of cultures corroborating their validity as a measure of culture.

At the same time, this research relied on collaborators from different countries who were willing and able to gather data at their universities. A collaborative network was established that allowed selecting four cultures that have been regarded as 
individualistic-oriented in the cross-cultural literature - namely Germany, Finland, New Zealand and the United Kingdom - and five cultures that tend to be categorized as more collectivistic - namely Brazil, India, Japan, Turkey and the Philippines (Hofstede, 1980; Schwartz, 2006b; Triandis, 2001). Figure 5.1 shows the distribution of these cultural samples along Schwartz' (2006) Autonomy-Embeddedness dimension which is conceptually related to individualism-collectivism (Inglehart, 2006; Kağıtçıbaşı, 1997). The selected individualistic samples are more centered at the Autonomy pole of the value orientation, whereas the collectivistic samples tend to be more spread along this dimension, but still cluster towards the Embeddedness pole.

A rule of thumb in cross-cultural studies is that the greater the number of cultures employed that can be ordered along a dimension, the more rival explanations can be ruled out for the phenomenon studied (Campbell, 1986). As a consequence, the conclusion that the observed differences between cultures can be explained with the theoretical dimension of interest is more convincing (van de Vijver \& Leung, 1997). Taking this into consideration, the sampling procedure employed in this study should fulfill this requirement. For instance, all sampled countries - regardless of whether they are characterized as individualistic or collectivistic - are located in geographically very different regions (Asia, Europe, Middle East, Oceania, and South America). If differences are found between the individualistic and collectivistic countries, that they are due to geographical distance can be ruled out, because almost all samples are located in very different regions. Furthermore, the collectivistic samples differed in terms of the predominant religion in their countries. According to the CIA World Factbook $^{15}$, the Philippines and Brazil are predominantly Christian countries. The main religion in Turkey is Islam, in India it is Hindu and in Japan it is Shinto and Buddhism. The individualistic samples were all from predominantly Christian countries. In terms of

\footnotetext{
${ }^{15}$ Retrieved June 2009, from https:/www.cia.gov/index.html
} 
social and economic development, the human development report ${ }^{16}$ indicates that among the highest ranking countries on the human development index (HDI) are both individualistic and collectivistic countries (Japan, Finland, New Zealand, U.K., Germany and Brazil). Turkey, India and the Philippines are classified as countries with medium human development.

\footnotetext{
${ }^{16}$ Retrieved June 2009, from http:/hdr.undp.org/en/statistics/
} 


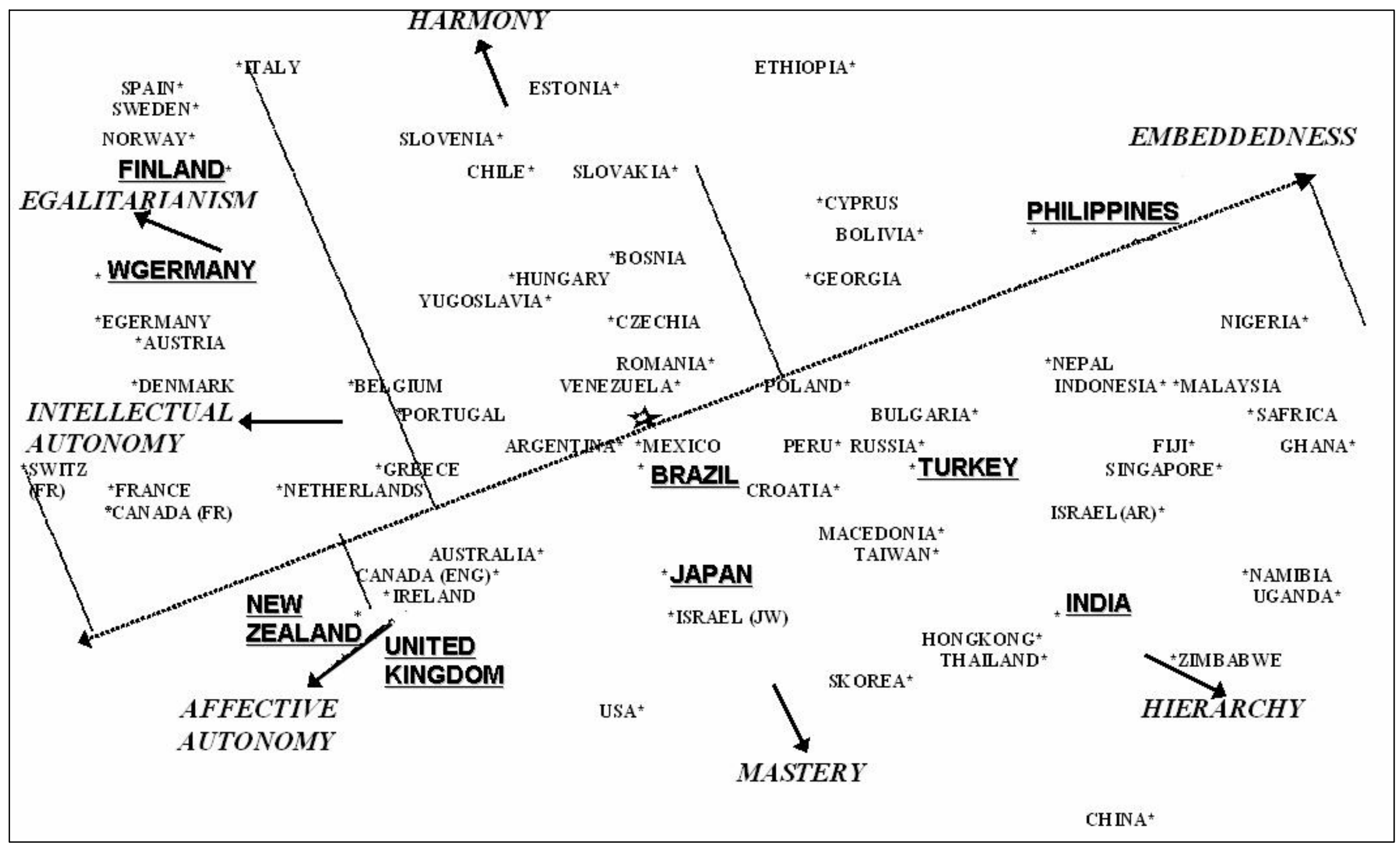

Figure 5.1: Co-plot map of 67 national groups on the value dimensionAutonomy-Embeddedness (adapted from Schwartz, 2006).

Note. Countries in bold and underlined were selected for the present study . 


\section{Method}

\section{Participants}

Ethics approval for this study was granted by the Victoria University of Wellington Human Ethics Committee. The author collected data in New Zealand in 2007. Collaborators in eight different countries (Brazil, Finland, Germany, India, Japan, the Philippines, Turkey and the United Kingdom) collected data at their universities between 2007 and 2009. The sample target was a total of at least 100 students preferably from the social sciences, with men and women equally balanced if possible. The sample from India had an insufficient number of participants, and thus, was excluded from the analysis. A total of 1,535 university students, predominantly with a major in social sciences, participated in this study. Respondents were only included in the analysis if they identified with the country of residence as assessed in the survey, reducing the total number of individuals to 1,456 (see Table 5.1 for more details on sample characteristics). Total sample size in each cultural group varied from 108 (U.K.) to 293 (Brazil). Even though a gender and age balance was aimed for in each cultural group, there were significant differences in the percentages of females $\left(\chi^{2}(7)=24.01, p\right.$ $<.001)$ and the average age $(F(7,1401)=52.00, p<.001)$. The Finnish sample showed the highest percentage of females (79.6\%) and the Turkish sample the lowest $(57.0 \%)$. Females were the majority in all cultural groups and made up $68 \%$ of the total sample. Respondents' average age was highest in Brazil $(M=27.35, S D=8.96)$ and lowest in the Philippines $(M=20.24, S D=1.41)$. The mean age of the total sample was 23.62 $(S D=6.02)$ 
Table 5.1: Sample characteristics of Study 3.

\begin{tabular}{|c|c|c|c|c|c|c|c|c|c|c|c|c|}
\hline \multirow[b]{2}{*}{ Country } & \multicolumn{4}{|c|}{ Total } & \multicolumn{4}{|c|}{ Version 1} & \multicolumn{4}{|c|}{ Version 2} \\
\hline & $\mathrm{N}$ & $\begin{array}{c}\text { Female } \\
(\%)\end{array}$ & Age (SD) & $\begin{array}{c}\text { Major in } \\
\text { Social } \\
\text { Sciences } \\
(\%)\end{array}$ & $\mathrm{N}$ & $\begin{array}{c}\text { Female } \\
(\%)\end{array}$ & Age (SD) & $\begin{array}{c}\text { Major in } \\
\text { Social } \\
\text { Sciences } \\
(\%)\end{array}$ & $\mathrm{N}$ & $\begin{array}{c}\text { Female } \\
(\%)\end{array}$ & Age (SD) & $\begin{array}{c}\text { Major in } \\
\text { Social } \\
\text { Sciences } \\
(\%)\end{array}$ \\
\hline Brazil & 293 & 71 & $27.35(8.96)$ & 66 & 141 & 67 & $27.20(9.11)$ & 58 & 152 & 74 & $27.50(8.85)$ & 75 \\
\hline Finland & 147 & 80 & $24.93(5.00)$ & 87 & 74 & 81 & $25.14(4.74)$ & 86 & 73 & 78 & $24.72(5.29)$ & 88 \\
\hline Germany & 169 & 76 & $26.68(6.24)$ & 67 & 68 & 75 & $26.92(6.54)$ & 64 & 101 & 76 & $26.52(6.06)$ & 69 \\
\hline Japan & 197 & 63 & $21.05(1.68)$ & 100 & 100 & 63 & $21.63(1.70)$ & 100 & 97 & 63 & $20.45(1.44)$ & 100 \\
\hline New Zealand & 175 & 66 & $23.69(5.98)$ & 72 & 88 & 73 & $23.13(5.30)$ & 78 & 87 & 59 & $24.27(6.60)$ & 66 \\
\hline The Philippines & 186 & 71 & $20.24(1.41)$ & 83 & 97 & 73 & $20.14(1.22)$ & 81 & 89 & 70 & $20.35(1.59)$ & 86 \\
\hline Turkey & 181 & 57 & $21.28(1.69)$ & 37 & 70 & 50 & $21.56(1.74)$ & 42 & 111 & 64 & $21.11(1.65)$ & 32 \\
\hline U.K. & 108 & 62 & $21.28(2.62)$ & 73 & 57 & 45 & $20.90(1.46)$ & 45 & 51 & 78 & $21.70(3.46)$ & 100 \\
\hline Totals & 1,456 & 68 & $23.62(6.02)$ & 73 & 695 & 66 & $23.57(5.91)$ & 69 & 761 & 70 & $23.66(6.12)$ & 77 \\
\hline
\end{tabular}

Note. SD denotes 'standard deviation'. Version 1 and Version 2 refer to the version of the survey containing different sets of Schwartz' values. 


\section{Procedure}

The majority of university students participated in the study to receive credits as part of a research participation requirement or an in-class exercise. Collaborators ensured that participants completed the survey silently and independently. Respondents from the U.K. completed the survey online.

The survey contained the 45 values that Schwartz recommended for crosscultural comparisons (Schwartz, 1994, 2006). The aim was to elicit ratings of each value on different response scales assessing the personal, moral and societal relevance of the values. A total of five different response scales for each value were constructed for this purpose. This means that by using all 45 values, participants would have needed to make 225 value ratings. To minimize participants' fatigue on this rather tedious task, the value survey was split into two versions. Versions 1 and 2 contained 22 and 23 value items respectively. Values were randomly selected from each of Schwartz' 10 motivational value types ensuring that all value types were represented in each version by an approximately equal number of values. Table 5.2 shows which values were included in which version of the questionnaire. Value items were presented in alphabetical order in the survey. Translations of the survey into other languages retained the value order of the English version.

The two versions were distributed randomly to the students. Regarding the age of participants, there was no significant interaction effect between cultural sample and version of the survey $(F(7,1393)=.84, p>.05)$. There were neither differences between the cultural sample and number of respondents per version $\left(\chi^{2}(7)=13.92, p>\right.$ $.05)$ nor the percentage of females per version $\left(\chi^{2}(7)=11.42, p>.05\right)$. See Table 5.1 for a summary of participants' characteristics completing the different versions of the survey. 
The instruction encouraged participants to answer the value section as quickly as possibly to elicit spontaneous and intuitive ratings of the values. It took about 30 minutes on average to complete the survey.

Table 5.2: Values included in Version 1 and Version 2 of the Moral Values Survey.

\begin{tabular}{|c|c|c|c|c|c|c|}
\hline & \multicolumn{3}{|c|}{ Survey Version 1} & \multicolumn{3}{|c|}{ Survey Version 2} \\
\hline & Value & Value & Value & Value & Value & Value \\
\hline & & Type & Orientation & & Type & Orientation \\
\hline 1. & $\begin{array}{l}\text { A world of } \\
\text { beauty }\end{array}$ & UN & STRAN & A varied life & ST & $\mathrm{OPCH}$ \\
\hline 2. & $\begin{array}{l}\text { Accepting } \\
\text { my portion }\end{array}$ & TR & CONS & A world at peace & UN & STRAN \\
\hline 3. & Ambitious & $\mathrm{AC}$ & SENH & Capable & $\mathrm{AC}$ & SENH \\
\hline 4. & $\begin{array}{l}\text { An exciting } \\
\text { life }\end{array}$ & ST & $\mathrm{OPCH}$ & Creativity & SD & $\mathrm{OPCH}$ \\
\hline 5. & Authority & $\mathrm{PO}$ & SENH & Curious & SD & $\mathrm{OPCH}$ \\
\hline 6. & Broadminded & UN & STRAN & Devout & $\mathrm{TR}$ & CONS \\
\hline 7. & $\begin{array}{l}\text { Choosing } \\
\text { own goals }\end{array}$ & SD & $\mathrm{OPCH}$ & Enjoying life & $\mathrm{HE}$ & $\mathrm{OPCH}$ \\
\hline 8. & Clean & SE & CONS & Family security & SE & CONS \\
\hline 9. & Daring & ST & $\mathrm{OPCH}$ & Forgiving & $\mathrm{BE}$ & STRAN \\
\hline 10. & Equality & UN & STRAN & Freedom & SD & $\mathrm{OPCH}$ \\
\hline 11. & Helpful & $\mathrm{BE}$ & STRAN & Humble & TR & CONS \\
\hline 12. & Honest & $\mathrm{BE}$ & STRAN & Influential & $\mathrm{AC}$ & SENH \\
\hline 13. & $\begin{array}{l}\text { Honouring } \\
\text { parents }\end{array}$ & $\mathrm{CO}$ & CONS & Loyal & $\mathrm{BE}$ & STRAN \\
\hline 14. & Independent & SD & $\mathrm{OPCH}$ & National security & SE & CONS \\
\hline 15. & Moderate & TR & CONS & Obedient & $\mathrm{CO}$ & CONS \\
\hline 16. & Pleasure & $\mathrm{HE}$ & $\mathrm{OPCH}$ & Polite & $\mathrm{CO}$ & CONS \\
\hline 17. & $\begin{array}{l}\text { Reciprocation } \\
\text { of favours }\end{array}$ & SE & CONS & $\begin{array}{l}\text { Preserving my } \\
\text { public image }\end{array}$ & $\mathrm{PO}$ & SENH \\
\hline 18. & $\begin{array}{l}\text { Respect for } \\
\text { tradition }\end{array}$ & TR & CONS & $\begin{array}{l}\text { Protecting the } \\
\text { environment }\end{array}$ & UN & STRAN \\
\hline 19. & $\begin{array}{l}\text { Self- } \\
\text { discipline }\end{array}$ & $\mathrm{CO}$ & CONS & Responsible & $\mathrm{BE}$ & STRAN \\
\hline 21. & Social justice & UN & STRAN & Social order & SE & CONS \\
\hline 22. & Successful & $\mathrm{AC}$ & SENH & Social power & PO & SENH \\
\hline 23. & Wealth & $\mathrm{PO}$ & SENH & Unity with nature & UN & STRAN \\
\hline 24. & - & - & - & Wisdom & UN & STRAN \\
\hline
\end{tabular}




\section{Questionnaire}

Individual-level Measures

Personal values. Schwartz $(1992,2007$ a) developed a 58 item value measure to assess the "personal" importance of values as guiding principles in people's life. Each value is followed by a defining phrase that further clarifies the meaning of the value. The personal importance ratings of values are elicited by instructing individuals to indicate "What values are important to ME as guiding principles in MY life?" The rating scale is composed of a 9-point Likert scale with the following labels: opposed to my values (-1), not important (0), (unlabeled; 2, 1), important (3), (unlabeled; 5, 4), very important (6) of supreme importance (7). Schwartz chose this asymmetric response scale with the reasoning that values are typically viewed as positive entities, usually ranging from somewhat to very important.

In the present study, only those 45 value items were used that Schwartz (1994, 2006) recommended for cross-cultural comparisons. The original response scale was changed into a 7-point bipolar scale defined by opposite poles (cf. Osgood, et al., 1957). This was done because values have been described as bipolar (Rokeach, 1973) and in order to match it to moral value ratings which were elicited with bipolar response scales (e.g. right vs. wrong). The bipolar anchors for the measurement of personal values were important to me and unimportant to me (IMP). The range of the scale was not numbered, but specified by the following qualitative labels (scale points for computation in brackets): extremely $(1,7)$, quite, $(2,6)$, slightly $(3,5)$ and neither one nor the other/ both equally (4) (see also Figure 5.2). 
To be HELPFUL (to work for the welfare of others) is ...

\begin{tabular}{|c|c|c|c|c|c|c|c|c|}
\hline & 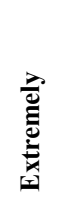 & 咅 & $\frac{\vec{D}}{\bar{E}}$ & 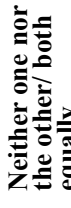 & 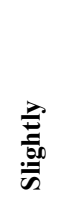 & $\stackrel{\mathscr{\Xi}}{\ddot{\Xi}}$ & 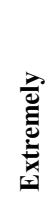 & \\
\hline ... important to me & 0 & 0 & 0 & 0 & 0 & 0 & 0 & ... unimportant to me \\
\hline $\begin{array}{l}\text {... something that I am } \\
\text { expected to strive for in my } \\
\text { society }\end{array}$ & O & $\mathrm{O}$ & 0 & 0 & 0 & $\mathrm{O}$ & $\mathrm{O}$ & $\begin{array}{l}\text {... something that is my free } \\
\text { choice to strive for in my } \\
\text { society }\end{array}$ \\
\hline ... a moral value to me & 0 & O & O & O & $\bigcirc$ & 0 & O & ... an immoral value to me \\
\hline ... something bad to follow & 0 & $\mathrm{O}$ & 0 & 0 & 0 & 0 & $\mathrm{O}$ & ... something good to follow \\
\hline$\ldots$ the right thing to strive for & 0 & O & O & 0 & 0 & 0 & O & $\ldots$ the wrong thing to strive for \\
\hline
\end{tabular}

Figure 5.2: Example of bipolar rating scales measuring five different evaluations of values.

Note. For the present analysis all response scales were recoded, except for 'bad vs. good' ratings.

In addition to ratings assessing the personal preferences of values, several additional 7-point bipolar response scales were constructed to elicit answers on the moral relevance of value items. Following the value terminology put forward in chapter two of this thesis, the following moral values were operationalized.

Personal moral values. In order to measure personal moral values, individuals were asked to rate each value on a bipolar response scale ranging from extremely immoral to me to extremely moral to me (MOR). Because of the ambiguity of the term 'moral' as pointed out in the introduction, two further bipolar response scales were constructed: (1) the right thing vs. the wrong thing to strive for (RIGHT), and (2) something bad vs. something good to follow (GOOD).

Societal moral values. These kinds of values were measured by asking individuals on a bipolar response scale to what extent they believed that they are expected to strive for the value in question in their society, versus to what extent it is 
their free choice to strive for it (EXP). This can be regarded as a direct measurement of the "cultural press" (Smith \& Schwartz, 1997, p. 111) to which people feel they are exposed.

Individuals were asked to rate each value on all five response scales. Figure 5.2 shows an example of the employed rating scales for the value "helpful" (see also Appendix $\mathrm{C}$ for the whole questionnaire). For all response scales, contextual cues were included where it was appropriate. For instance, the expectation response scale may refer to expectations of family, co-workers, friends and whatever relevant social group comes to mind. Including the contextual cue of 'societal expectations' ensures that individuals employ the same 'frame of reference' (cf. Lievens, De Corte, \& Schollaert, 2008) when answering the value items. Prior to data collection, the response scales had been discussed in individual sessions with respondents from a culturally diverse background in regard to their comprehensibility. They were modified in their wording if necessary.

Moral attitudes. Items assessing individuals' moral attitudes were obtained from the Morally Debatable Behaviour Scale (MDBS, Harding \& Phillips, 1986). Study 2 in chapter three of this thesis has already shown that there are pronounced cultural differences in attitudes towards matters of life and death, labelled as personal-sexual issues. Hence, the same items that were used in Study 2 to assess these moral attitudes were included in the present survey. The response scale assessed the 'wrongness' of the respective behaviours and ranged from not wrong at all (1) to extremely wrong (5) (see also Appendix C).

Demographic Variables. The questionnaire also included the usual set of demographic questions (e.g. age and gender). Respondents were also asked to rate their religiosity on a 5-point Likert scale ranging from $1=$ 'not important' to $5=$ 'very 
important'. Their political orientation was measured on a 7-point Likert scale ranging from $1=$ 'extremely liberal' to $5=$ 'extremely conservative'.

\section{Translation}

The questionnaire was developed in English. Since the value items came from the SVS which has been translated into 47 languages (Schwartz, 2006a), established translations were used for them. Translations of the Morally Debatable Behaviour Scale were obtained from the World Value Survey webpage ${ }^{17}$. The English version of the remaining parts of the questionnaire was translated into German and Portuguese by the author and its accuracy was verified with at least one other bilingual colleague. Collaborators from Finland, Japan and Turkey translated the survey into the local language. For all translations a 'committee approach' was employed in which the translation was discussed within a group of bilinguals (Harkness, 2003). The Filipino sample received the English version, since English is one of the official languages in the Philippines.

\section{Culture-level Value Measures}

Personal values (IMP ratings), personal moral values (RIGHT, GOOD, MORal ratings) and societal moral values (EXPectation ratings) were aggregated at the culturelevel to obtain a measure of these values from each cultural sample. This was done by computing the cultural sample means of each value item rated on the respective response scale.

\footnotetext{
${ }^{17}$ Retrieved October 2008, from http://www.worldvaluessurvey.org/
} 


\section{Analytical Strategy}

\section{Data Screening}

The value part of the survey was screened first for univariate outliers and then for missing values. The value survey contained a relatively large number of items due to the fact that each value was rated on five different response scales. Some of the response scales were phrased reversely to counteract possible response tendencies to rate every item in a similar fashion. It was anticipated that during this cognitively demanding task, participants might make mistakes by confusing the poles of the rating scales. If so, these responses should be detectable as univariate outliers. Outliers are observations that show an unusually high or low value on a variable that make the observation stand out from the others (Hair, Black, Babin, Anderson, \& Tatham, 2006). They distort statistics and lead to Type I and Type II errors (Tabachnick \& Fidell, 2007). Univariate outliers defined as exceeding 2.5 standard scores (cf. Hair, et al., p. 75) were removed from the analysis on a pairwise basis. Across all value items, these constituted on average $1.52 \%$ of all responses (Version 1 and Version 2 combined) of the survey ${ }^{18}$.

Schwartz (2007a) highly recommended dropping respondents from the analysis who show too many missing values. Hence, participants with more than $30 \%$ of value responses missing were excluded listwise. They constituted $3.5 \%$ of the total sample. Loss of cases to an extent of less than $5 \%$ due to missing data can be regarded as small (cf. Graham, 2009).

\footnotetext{
${ }^{18}$ Preliminary analyses using multidimensional scaling on the cultural value type hierarchies (Version 1 of the survey) showed that the results plotted in a two-dimensional space were very similar with and without outliers. Pearson correlations between the two solutions were .98 (for dimension 1) and .86 (for dimension 2).
} 


\section{Reliabilities}

A preliminary analysis was conducted to examine whether measures assessing values and moral attitudes have satisfying psychometric properties in all samples.

Values. The primary interest lay in examining whether Schwartz' value items possessed adequate internal consistencies across cultural samples and response scales. Hence, reliability indices were computed by averaging across value types and value orientations respectively. This was done for each version separately.

Schwartz (1992) reported that country-specific reliabilities averaged across value types ranged from .67 (in Australia) to .71 (in Israel). He argues that these reliabilities are reasonable considering the small number of items in each index. More recently, Schwartz (2003) reported reliabilities of a shortened value survey (21 items: the Portrait Values Questionnaire, PVQ) employed in the European Social Survey. The PVQ is an alternative to the SVS and includes verbal portraits of 40 different people to measure personal values in samples less accustomed to abstract and context-free thinking (Schwartz, Melech, Lehmann, Burgess, \& Harris, 2001). Preliminary analyses of the short version showed that the alpha reliabilities were low (e.g. .49 for selfdirection in a combined U.K. and Netherlands sample), however, it was still possible to show that the value types predicted behavior and attitudes systematically (Schwartz, 2003).

Cronbach's alpha (Cronbach, 1951) is typically lower for short scales (Cortina, 1993). In these cases it is more appropriate to report the mean inter-item correlation (mean $\mathrm{r}_{\mathrm{ij}}$ ). Considering that the two value surveys in the present study contained value types that did not consist of more than four items (see Table 5.2.), the mean itemintercorrelation was regarded as a more adequate gauge for assessing their reliabilities. The optimal range for mean $r_{i j}$ has been recommended to be between .20 and .40 (Briggs \& Cheek, 1986). Reliabilities of the four higher order value orientations, 
containing somewhat more value items than the ten value types, were assessed with the commonly employed Cronbach alpha. Relatively low reliabilities were still expected due to the small number of items ranging from four (self-enhancement) to seven (conservation). Based on Schwartz' $(1992,2003)$ findings, alphas greater than .50 were regarded as acceptable.

Moral attitudes. The reliability of the moral attitude measure across cultural samples was assessed with a multigroup confirmatory factor analysis (CFA) in AMOS 6. Despite having already evaluated the factor structure of this scale in Study 2 of this thesis, there were considerable differences between the previous and the present study: the sample of this study consisted of university students only, the response scale had been modified, and the focus lay on examining the cross-cultural reliability of the personal-sexual factor only.

Moral attitude items were the same in Version 1 and Version 2 of the present survey, therefore, individuals from the same country were grouped together across survey versions. This increased the sample size, so that running a multi-group CFA was permissible. Similarly to Study 2, missing values were deleted listwise.

The first step in conducting the CFA consisted of testing the fit of the one-factor model in the pooled within-country data. The pooled within-group covariance matrix served as data input. This procedure weighs the correlation matrices of each country equally so that they have the same influence on the overall matrix (Fontaine \& Fischer, in press; Muthén, 1994). The results of the pooled within-group CFA provide a general picture of the average solution across all countries in terms of model fit and estimated regression weights. Errors were not allowed to be correlated in the model. The second step consisted of testing cross-cultural equivalence by conducting a multigroup CFA. The final aim was to test metric invariance of the scale, i.e. that the factor loadings are similar across cultural samples (Chen, 2008; Cheung \& Rensvold, 2000). A well-fitting 
model across samples would justify the computation of a factor mean and therefore its use as a dependent variable in subsequent analyses.

\section{Analysis of Value Type Hierarchies}

Mean ratings of the ten value types were computed for the different response scales and for all cultural samples. Mean ratings were also computed across all samples to obtain a pan-cultural baseline of value endorsement (cf. Schwartz \& Bardi, 2001).

The first analysis focused on the pan-cultural findings. The aim was to assess whether there are differences and similarities in value hierarchies derived from different response scales. Multidimensional scaling $\left(\mathrm{MDS}^{19}\right)$ was conducted for this purpose. MDS is a useful alternative to factor analysis for culture-level data (Chinese Culture Connection, 1987), since the latter requires a data point to item ratio of 10:1 (Nunnally, 1978) which is usually not fulfilled with aggregated datasets. The pan-cultural valuetype hierarchies, obtained with different response scales, served as data input.

The second analysis focused on the culture-specific value ratings. MDS was again performed, this time across response scales and cultural groups. The aim was to confirm the similarities and differences in response scales even after taking into account culture as a variable. Response scales that are located very closely to each other in the map indicate highly similar value type hierarchies and are therefore redundant for subsequent analyses. Furthermore, this kind of analysis allows identifying similarities and differences between cultural samples. It was expected that samples categorized a priori as individualistic or collectivistic would cluster together according to this distinction.

\footnotetext{
${ }^{19}$ See chapter 2, study 1 , for more technical details on MDS.
} 


\section{Multilevel Regression Analysis}

A multilevel regression analysis was conducted to examine the effect of different values (personal values, personal moral values and perceived societal expectations) at the individual- and culture-level on individuals' moral attitudes. Similarly to chapter three (Study 3), the interest lay in examining the impact of broad cultural dimensions on moral attitudes. Hence, Schwartz' higher order value orientations were taken for this purpose. Only the value orientations openness-tochange versus conservation were examined, because hypotheses based on cultural theories and previous empirical findings (e.g. Study 2, chapter three) were derived only for these kinds of values. Country scores for openness-to-change and conservation were computed by aggregating the value items at the culture-level and calculating the mean of the scores for the items that index the respective higher order value orientation. A single country score was then obtained by subtracting the score for conservation (negative pole of the bipolar value dimension) from openness-to-change (positive pole of the bipolar value dimension). This procedure reduces the likelihood of multicollinearity of opposite value types, which are supposed to correlate highly negatively with each other (cf. Schwartz, 2007a).

Hierarchical linear modelling (HLM, Raudenbush \& Bryk, 2002) was used in chapter three to conduct the multilevel regression analysis. HLM is the method of choice for estimating cross-level relationships. It is a sophisticated statistical procedure that estimates parameters on the different levels of analysis simultaneously. As such, it takes into account the standard error estimates associated with the various levels of analysis. The drawback is that a large number of data points are necessary at each level. Rules of thumb have been proposed for a two-level model ranging from 30 observations at both level 1 and level 2 to a ratio of 50/20 or 100/10 (see Matsumoto \& Yoo, 2006). The data for the present study comprising eight cultural groups do not meet these 
minimum requirements. A solution is to use a regression method that is conceptually identical to HLM, but does not require large scale data input. CLOP (Cross-Level OPerator, James \& Williams, 2000) is a variant of multiple regression analysis that can be used to test cross-level effects in SPSS with small number of units. In a CLOP analysis, individual-level scores are substituted by unit-level scores. This was done in the present study by assigning all individuals within a given cultural group the group's score on the respective predictor. Hence, individuals from the same cultural group obtained the same score for the higher level predictor variable.

The results of HLM and CLOP analysis are similar in terms of the estimated regression coefficients. However, a major difference is that CLOP does not partition variance into within- and between-unit components. This has important implications, such as higher level predictors typically showing smaller effect sizes than they would show with HLM (Klein, et al., 2000). Nevertheless, CLOP is a good alternative if sample size is not sufficient for HLM (James \& William, 2000). Fischer (2008b), for example, used CLOP in an analysis involving four countries to predict the use of the seniority principle (individual-level) in organizations with Hofstede's culture-level values.

\section{Cross-validation}

All analyses were first conducted with Version 1 of the survey. Data from the second version served as a cross-validation sample. Datasets for the two versions differed from each other in terms of participants and value items. Nevertheless, participants were from the same countries across the two versions and value items included in the two versions were from the same value types. Results that are replicated across the two versions, despite these differences, would point to robust findings. 


\section{Results and Discussion}

\section{Confirmatory Factor Analysis on Moral Attitudes}

The one-factor model for the pooled within-sample yielded a significant chisquare statistic $\left(\chi^{2}(5)=51.35, p<.001\right)$ which is common with large sample sizes (Bentler, 1992a) ${ }^{20}$. However, other commonly used fit indices suggested that the model fitted the data well (see $\mathrm{Hu} \&$ Bentler, 1999, for cutoff values): CFI was .97 and RMSEA was .08. The factor loadings were all significant and sizeable (.54-.72).

Next, the model was tested across all eight cultural groups, first without any cross-country constraints. This is the most basic level of measurement invariance, known as configural invariance (Chen, 2008; Cheung \& Rensvold, 2000). The results showed again a significant chi-square $\left(\chi^{2}(40)=120.49, p<.001\right)$. The values for CFI and RMSEA (.95 and .04) indicated that the one-factor structure fitted relatively well across all eight countries. Next, equality constraints were imposed on the factor loadings. This is the second level of measurement invariance, known as metric invariance (Chen, 2008). The CFI dropped to .90, but RMSEA still indicated a good fit with a value of .04. Considering that the fit criteria were developed for evaluating single-group models, it may be acceptable to relax the criterium for CFI somewhat (Browne \& Cudeck, 1992) and to regard the values here as acceptable. On the basis of this analysis, mean scores were computed composed of the moral attitude items.

\footnotetext{
${ }^{20}$ For the sake of consistency in rejecting the chi-square test as indicator of model fit, nested models in subsequent analyses were not compared on the basis of their chi-squares as it is usually done.
} 


\section{Reliabilities of Value Measures}

Mean inter-item correlations (Mean $\mathrm{r}_{\mathrm{ij}}$ ) averaged across value types are displayed in Table 5.3 for the pan-cultural and culture-specific samples and for all response scales. The indices are shown separately for Version 1 and Version 2 of the survey. The mean $r_{i j}$, were of acceptable size (cf. Briggs \& Cheek, 1986) for both the pan-cultural and culture-specific samples and for all response scales. The lowest mean $\mathrm{r}_{\mathrm{ij}}$ was .21 for 'importance' ratings in the Philippines (Version 1) and the highest was .51 for 'societal expectation' ratings in Japan (Version 1). Even though there was some variation in mean $r_{i j}$, there was no evidence that a specific cultural sample yielded consistently higher or lower mean $\mathrm{r}_{\mathrm{ij}}$. In order to compare how well the newly constructed response scales perform in relation to the original 'IMPortance rating' scale, the pan-cultural mean $\mathrm{r}_{\mathrm{ij}}$ can be inspected. It appears that the new scales perform as well as the 'IMPortance rating' scale or even a bit better, as in the case of the 'societal expectation' scale.

Cronbach alphas averaged across higher order value orientations are displayed in Table 5.4 for the pan-cultural and culture-specific samples and for all response scales. Cronbach alphas for all value orientations were acceptable ranging from .51 ('good' ratings in Brazil, Version 1) to .77 ('societal expectation' ratings in Japan, Version 1). Again, there was no evidence that a specific cultural sample yielded consistently higher or lower reliabilities or that the 'moral value' rating scales performed poorer than the 'IMPortance' scale. 
Table 5.3: Individual-level mean item-intercorrelation (mean $r_{i j}$ ) for each sample and response scale averaged across value types.

\begin{tabular}{|c|c|c|c|c|c|c|c|c|c|c|}
\hline \multirow[b]{2}{*}{ Sample } & \multicolumn{5}{|c|}{ Version 1} & \multicolumn{5}{|c|}{ Version 2} \\
\hline & IMP & EXP & MORAL & RIGHT & GOOD & IMP & EXP & MORAL & RIGHT & GOOD \\
\hline Pan-cultural & .31 & .39 & .37 & .30 & .29 & .30 & .35 & .40 & .34 & .34 \\
\hline Brazil & .24 & .29 & .26 & .22 & .24 & .27 & .35 & .35 & .30 & .32 \\
\hline Finland & .24 & .34 & .28 & .28 & .27 & .23 & .29 & .31 & .28 & .30 \\
\hline Germany & .27 & .39 & .33 & .25 & .26 & .22 & .29 & .24 & .26 & .29 \\
\hline Japan & .30 & .51 & .30 & .24 & .27 & .29 & .27 & .32 & .31 & .26 \\
\hline New Zealand & .27 & .42 & .32 & .32 & .22 & .24 & .35 & .31 & .30 & .30 \\
\hline The Philippines & .21 & .32 & .31 & .33 & .33 & .27 & .35 & .40 & .34 & .34 \\
\hline Turkey & .33 & .40 & .35 & .35 & .31 & .29 & .36 & .37 & .29 & .31 \\
\hline U.K. & .38 & .23 & .32 & .41 & .37 & .24 & .35 & .29 & .20 & .23 \\
\hline
\end{tabular}

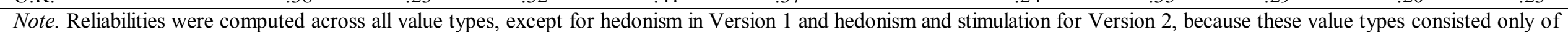

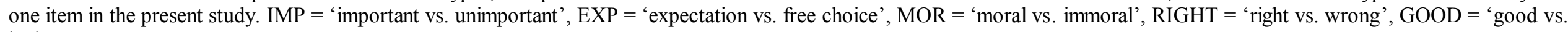
bad'.

Table 5.4: Individual-level Cronbach alphas for each sample and response scale averaged across value orientations.

\begin{tabular}{|c|c|c|c|c|c|c|c|c|c|c|}
\hline \multirow[b]{2}{*}{ Sample } & \multicolumn{5}{|c|}{ Version 1} & \multicolumn{5}{|c|}{ Version 2} \\
\hline & IMP & EXP & MORAL & RIGHT & GOOD & IMP & EXP & MORAL & RIGHT & GOOD \\
\hline Pan-cultural & .63 & .73 & .72 & .66 & .64 & .69 & .67 & .66 & .66 & .67 \\
\hline Brazil & .54 & .65 & .60 & .58 & .51 & .58 & .57 & .56 & .56 & .57 \\
\hline Finland & .61 & .69 & .56 & .66 & .63 & .63 & .62 & .64 & .63 & .63 \\
\hline Germany & .61 & .75 & .66 & .56 & .62 & .65 & .62 & .61 & .62 & .62 \\
\hline Japan & .61 & .77 & .64 & .66 & .69 & .69 & .67 & .67 & .68 & .68 \\
\hline New Zealand & .59 & .69 & .64 & .61 & .56 & .62 & .61 & .60 & .60 & .61 \\
\hline The Philippines & .57 & .70 & .65 & .69 & .67 & .68 & .67 & .67 & .67 & .67 \\
\hline Turkey & .65 & .70 & .70 & .69 & .65 & .68 & .68 & .67 & .67 & .68 \\
\hline U.K. & .66 & .63 & .67 & .64 & .67 & .65 & .65 & .65 & .66 & .65 \\
\hline
\end{tabular}

Note. IMP = 'important vs. unimportant', EXP = 'expectation vs. free choice', MOR = 'moral vs. immoral', RIGHT = 'right vs. wrong', GOOD = 'good vs. bad'. 


\section{Differences and Similarities of Response Scales and Cultural Groups}

\section{The Pan-cultural Value Type Hierarchy}

The differences and similarities of pan-cultural value hierarchies produced by different response scales (see rank orders in Appendix D) were graphically displayed through MDS. With five elements to display (here the five response scales), there were 5-1 possible dimensions as solutions (Hair, et al., 2006). A suggested guideline is to have more than four times as many objects to display as dimensions desired (cf. Hair, et al., 2006, p. 645). Hence, at least five objects are required for a one-dimensional map. Therefore, a one-dimensional solution was most appropriate for the present data. Tucker's coefficient of congruence is an indicator of the fit of the solution with higher fit measures (to a maximum of 1) indicating better solutions. The fit for the present solution was high with a value of .99 .

The perceptual map can be seen in Figure 5.3. A clear feature is that personal values (IMP) and perceived societal expectations (EXP) are located opposite to each other. In MDS, spatial distance is a measure of association between objects. Hence, the further apart two objects, the more dissimilar they are to each other. Inspecting Figure 5.3 it becomes clear that EXPectation and IMPortance ratings produce different value hierarchies whereas judgments of right and wrong and good and bad were more closely related to IMPortance ratings. Personal moral value ratings were located in-between societal expectations and personal preferences. Spearman correlations with Schwartz and Bardi's (2001) pan-cultural value hierarchies based on students' personal values (see Appendix D) showed that: (1) IMPortance, GOOD, RIGHT and to some extent also MORal ratings produced value hierarchies very similar to Schwartz and Bardi's pancultural value hierarchy $\left(r_{s}(9)_{\mathrm{IMPortance}}=.95, p<.001 ; r_{s}(9)_{\mathrm{GOOD}}=.94, p<.001 ; r_{s}\right.$ $\left.(9)_{\mathrm{RIGHT}}=.93, p<.001 ; r_{s}(9)_{\mathrm{MORal}}=.86, p<.01\right)$; (2) EXPectation value hierarchies were different from Schwartz and Bardi's pan-cultural value hierarchy $\left(r_{s}(9)=.55, p>\right.$ 
.05). Therefore, there seems to be some overlap between what people actually desire and the personal moral obligations they perceive, but not between desires and societal expectations.

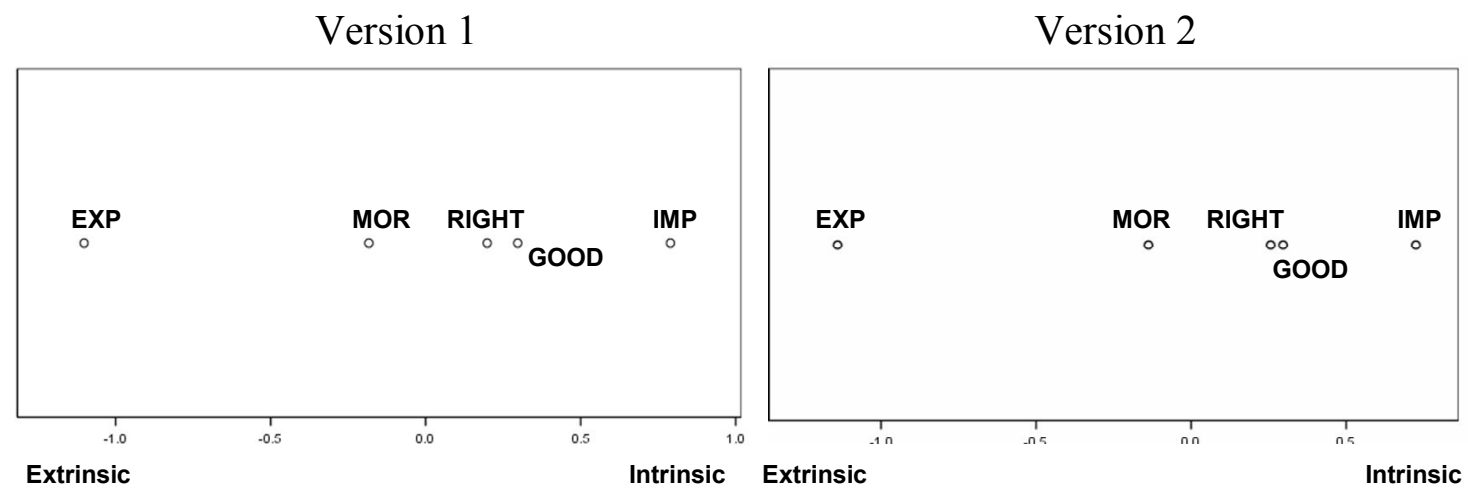

Figure 5.3: One-dimensional MDS representation of value hierarchies using five different response scales (pan-cultural sample, Version 1 and 2 of the Survey).

Note. $\mathrm{EXP}=$ 'expectation vs. free choice response scale', $\mathrm{MOR}=$ 'moral vs immoral response scale', RIGHT $=$ right vs. wrong response scale, GOOD $=$ good vs. bad response scale, IMP $=$ important vs. unimportant response scale.

\section{Culture-specific Value Type Hierarchies}

$M D S$. In order to examine associations of both response scales and cultural samples, a MDS was conducted across these variables. The scree plot indicated a twodimensional solution. Tucker's coefficients of congruence for the one- to fourdimensional solution were $.97, .99, .99$, and .99 . Adding more dimensions than just two did not substantially affect the Tucker's coefficients. Hence, a two-dimensional solution was retained. The map is depicted in Figure 5.4. Similarly to the pan-cultural analysis, there is a polarization between rating of values as societal expectations and personal preferences. 'GOOD' and 'RIGHT' evaluations are located in the middle of dimension 1. Furthermore, there is a relatively strong partition of hypothesized individualistic and collectivistic samples on dimension 2. Individualistic samples (Germany, Finland, New Zealand and the U.K.) tend to be clustered in the upper part of the two-dimensional space. Collectivistic samples (Brazil, Japan, the Philippines and Turkey) tend to cluster 
around the lower part of the map. Hence, dimension 2 appears to reflect the expected individualism- collectivism distinction of the cultural samples.

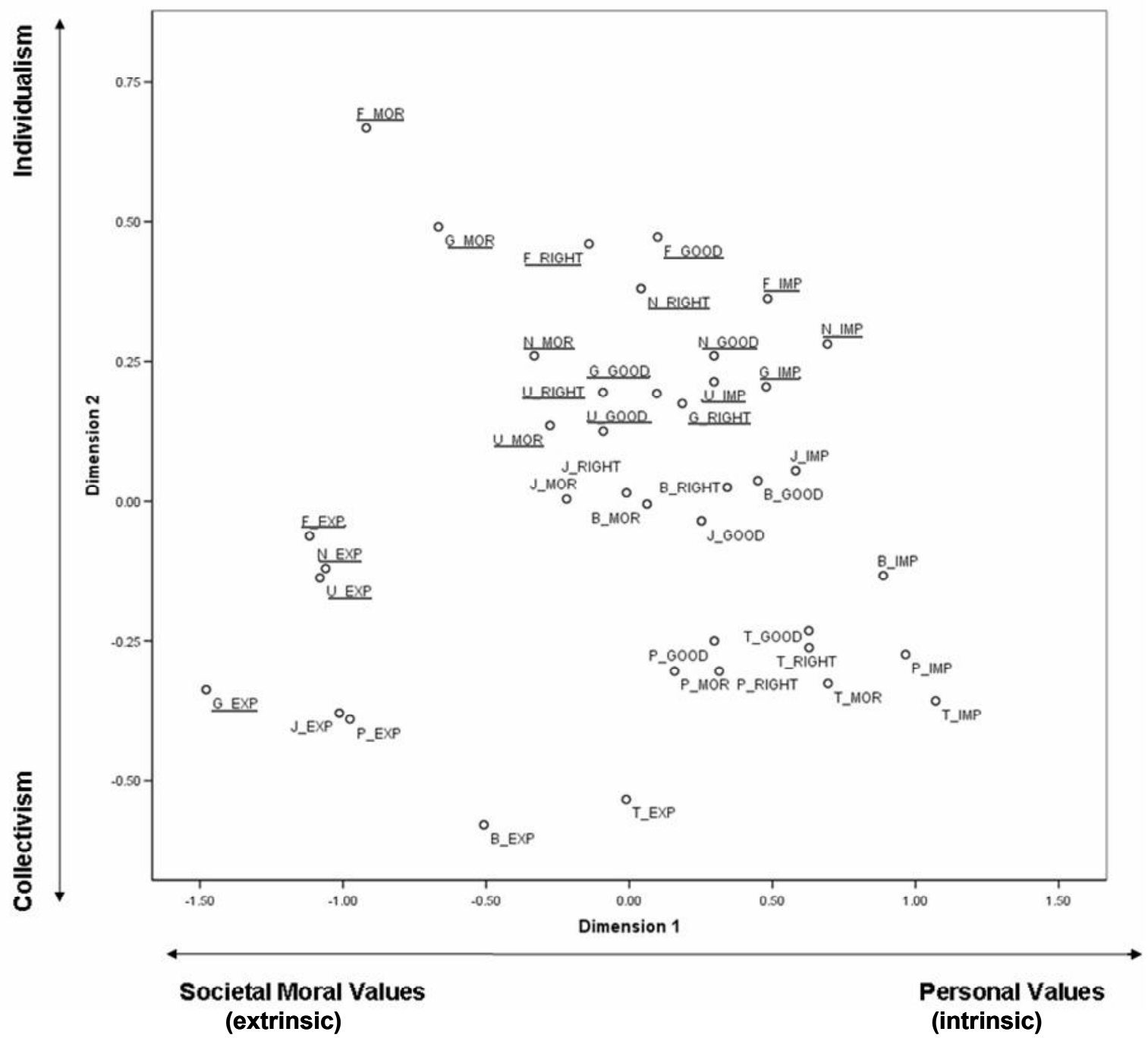

Figure 5.4: Two-dimensional MDS representation of response scales and cultural

samples.

Note. Underlined objects denote individualistic cultural samples according to the literature. $\mathrm{B}=$ Brazil, $\mathrm{F}$ $=$ Finland, $\mathrm{G}=$ Germany, $\mathrm{J}=$ Japan, $\mathrm{N}=$ New Zealand, $\mathrm{P}=$ the Philippines, $\mathrm{T}=$ Turkey, $\mathrm{U}=$ United Kingdom, EXP = 'expectation vs. free choice' response scale, MOR = 'moral vs. immoral' response scale, RIGHT $=$ right vs. wrong' response scale, GOOD $=$ 'good vs. bad' response scale, IMP $=$ 'important vs. unimportant' response scale. 
Follow-up Analyses. This strong partition into individualistic and collectivistic samples may be due to the effect of cultural response styles, or response sets. A response set is the systematic tendency to respond to a range of items on some basis other than the item content (Paulhus, 1991). There are two response styles which have caught the attention of cross-cultural psychologists: extreme response style (ERS) and acquiescence response style (ARS, Cheung \& Rensvold, 2000). ERS is the tendency to use the extremes of a rating scale. ARS occurs when individuals from one group systematically give higher responses than another group. There is empirical evidence for cross-cultural differences in ARS, with consistent evidence now that an ARS tendency is more pronounced in collectivistic-oriented cultures (e.g. Fischer, Fontaine, van de Vijver, \& van Hemert, in press; Johnson, Kulesa, Llc, Cho, \& Shavitt, 2005; Smith, 2004; Smith \& Fischer, 2008). According to van de Vijver and Leung (1997) response styles constitute a method bias and are part of measurement error. Hence, they should be detected and controlled for. In a similar vein, Schwartz (1992) recommended applying a centering procedure in which means are adjusted in order to eliminate national differences in the use of the response scale. Schwartz and Bardi's (2001) pan-cultural value hierarchy, for instance, is based on centered means. In order to find out whether there is indeed a possible response style, mean ratings across all value items were computed for each response scale and for the individualistic sample, the collectivistic sample and the combined pan-cultural sample. Figure 5.5 displays the results and it appears that the collectivistic samples indeed showed the tendency to score systematically higher than the pan-cultural mean and the individualistic sample, which can be interpreted as a sign of acquiescence. 


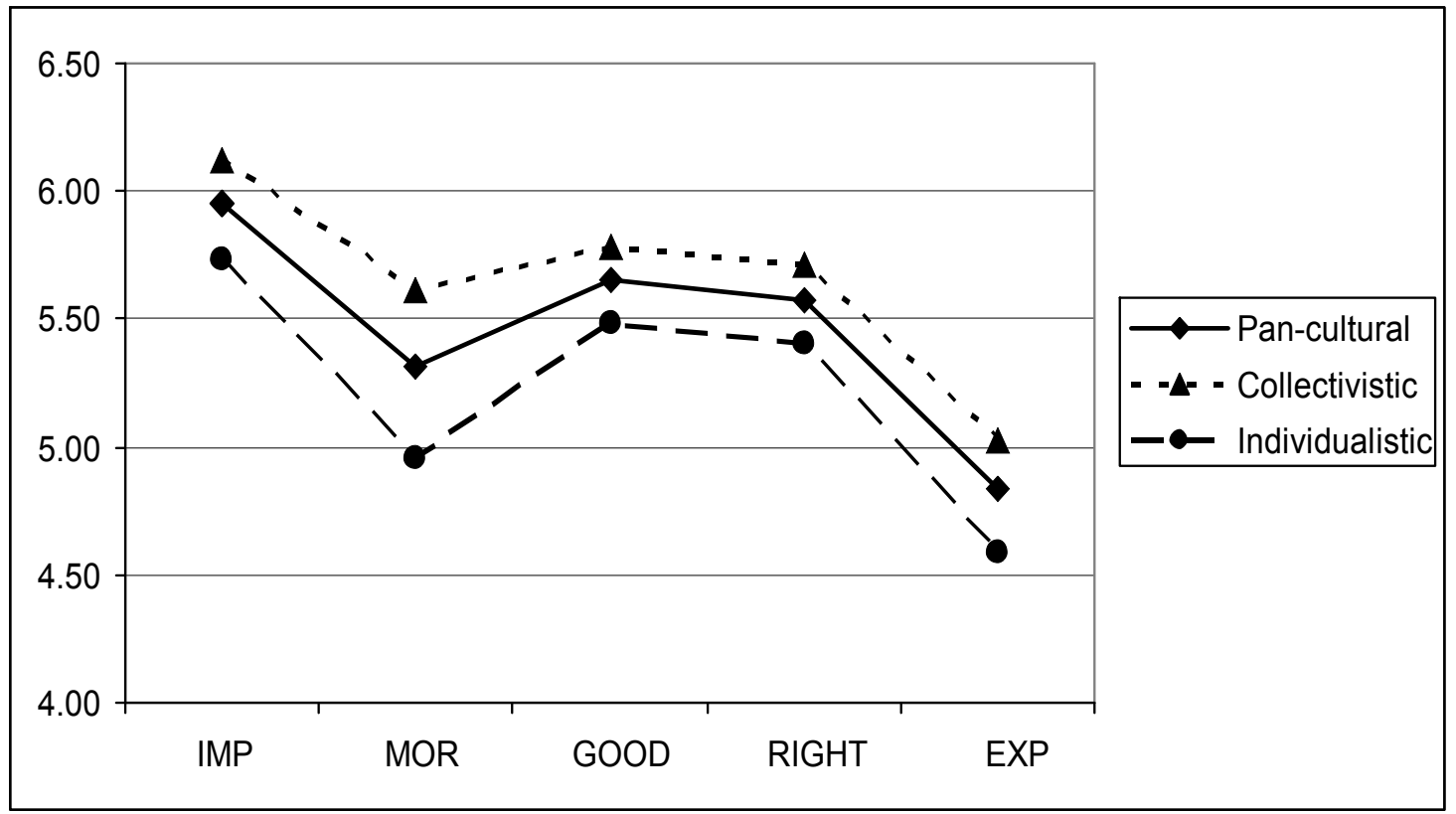


correlated at $.37(p<.05)$. Spearman correlations were very $\operatorname{similar}\left(r_{s \operatorname{dim} 1}(39)=.63, p\right.$ $\left.<.001, r_{s \operatorname{dim} 2}(39)=.44, p<.01\right)$. What can be seen from Appendix $\mathrm{E}$ is that there is still an individualism/ collectivism distinction in dimension one which is especially pronounced for IMPortance and EXPectation ratings, but less evident for MORal, GOOD and RIGHT ratings. Hence, there is some overlap between the original rating and the transformed ranking solution, especially for the distinction of the different response scales. However, this overlap is far from being perfect, for dimension two in particular. Hence, acquiescence may be a confounding variable in the detection of cultural differences between individualistic and collectivistic samples in the present study. Therefore, for subsequent analyses, the mean ratings were corrected for scale use following Schwartz' (1992, 2007a) recommended procedure for aggregated culturelevel data: the pan-cultural mean on all values was computed, the cultural sample mean was subtracted from the pan-cultural mean, and the result was added to the score of each value dimension. This was done separately for each response scale.

To conclude, regardless of whether the value type ratings or rankings were analyzed, the response scales IMPortance and EXPectation yielded different findings. The analyses have also shown that the GOOD as well as RIGHT evaluation is relatively similar to the IMPortance value assessment. Hence, the former two response scales appeared to be redundant and were dropped from subsequent analyses. Yet, the MORal ratings were retained because of their ambiguous position in between IMPortance and EXPectation ratings (Figure 5.3. and 5.4, Appendix E) as well as the theoretical reason that personal values and personal moral values may constitute separate constructs (see chapter two).

The Content of Moral Values. A comparison of individualistic and collectivistic samples regarding the content of their personal moral and perceived societal moral values can be seen in Figure 5.6. Because of the issue of response sets, the value ratings 
of the ten value types were transformed into rank orders in order to be able to compare them across cultures. What Figure 5.6 shows is that individualistic and collectivistic samples are very similar in their value profile in regard to personal moral values. This is also reflected in the mean Spearman correlation for personal moral value hierarchies across cultural samples which was .90 . The values that were mostly rated as personal moral values were of the benevolence ( $1^{\text {st }}$ rank), universalism $\left(2^{\text {nd }}\right.$ and $3^{\text {rd }}$ rank) and conformity ( $3^{\text {rd }}$ and $2^{\text {nd }}$ rank) value type. As least moral were judged values belonging to the value types of power $\left(10^{\text {th }}\right.$ rank $)$, hedonism $\left(9^{\text {th }}\right.$ rank in collectivistic sample) and tradition $\left(9^{\text {th }}\right.$ rank in individualistic sample) and stimulation $\left(8^{\text {th }}\right.$ rank $)$.

Societal moral value ratings showed greater variability between individualistic and collectivistic samples. This is also reflected in the mean Spearman correlation for societal moral value hierarchies across cultural samples which was .69. Individualistic samples perceived achievement $\left(1^{\text {st }}\right.$ rank), self-direction $\left(2^{\text {nd }}\right.$ rank), and benevolence $\left(3^{\text {rd }}\right.$ rank) as most salient. Collectivistic sample on the other hand perceived conformity $\left(1^{\text {st }}\right.$ rank), security $\left(2^{\text {nd }}\right.$ rank), and also benevolence ( $3^{\text {rd }}$ rank) as the most salient values. There was more concurrence in regard to the least ranking value types: hedonism and stimulation $\left(10^{\text {th }}\right.$ and $9^{\text {th }}$ rank) as well as tradition $\left(8^{\text {th }}\right.$ rank) were at the bottom of the rank order. Hence, values associated with a personal focus were more salient as a societal expectation in the individualistic sample, whereas values with a social focus were more relevant in the collectivistic sample. 


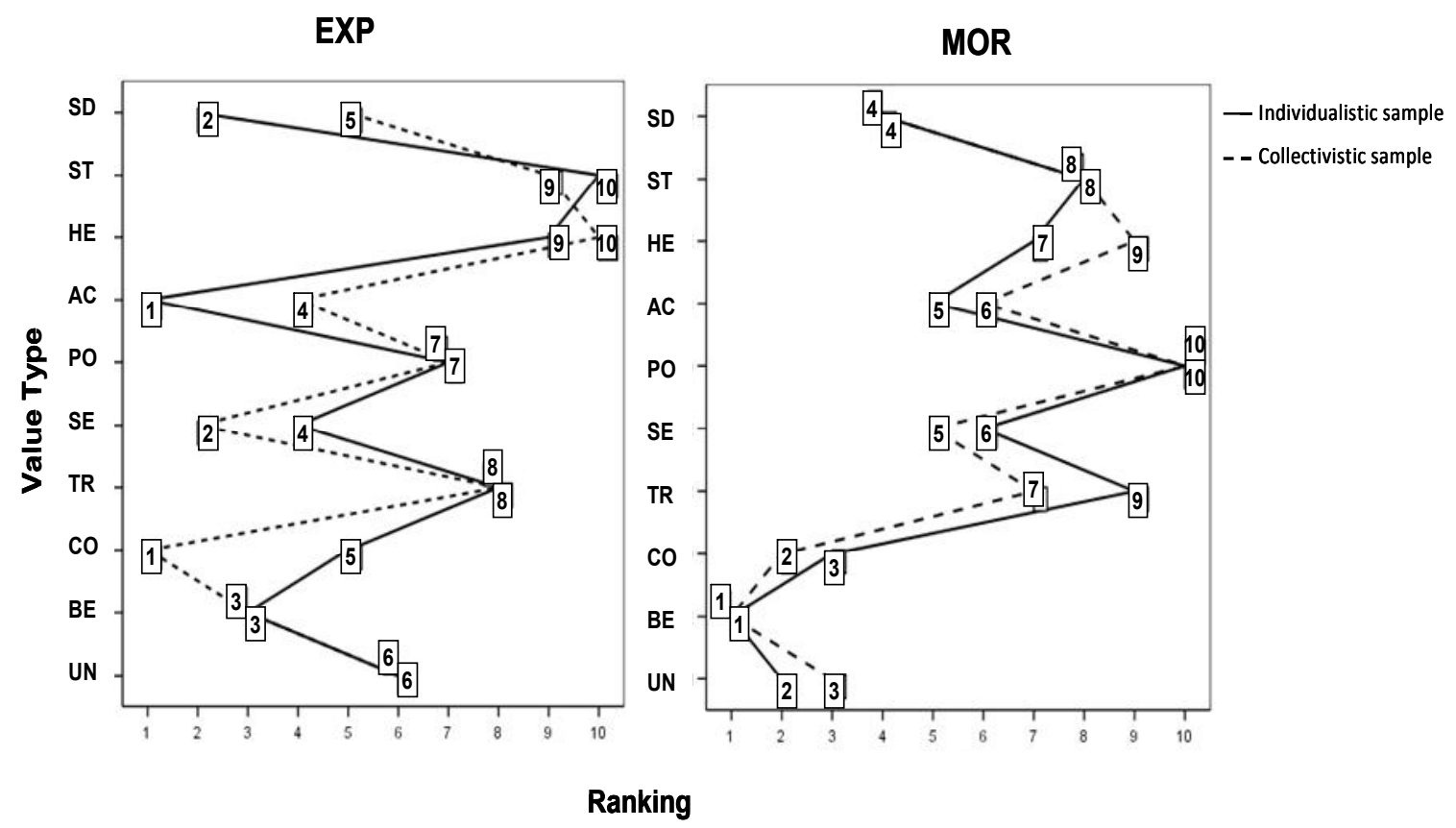

Figure 5.6: Value ranking profile of individualistic and collectivistic samples for societal moral values (societal expectation ratings) and personal moral values (moral value ratings).

Note. $\mathrm{SD}=$ self-direction, $\mathrm{ST}=$ stimulation, $\mathrm{HE}=$ hedonism, $\mathrm{AC}=$ achievement, $\mathrm{PO}=$ power, $\mathrm{SE}=$ security, $\mathrm{TR}=$ tradition, $\mathrm{CO}=$ conformity, $\mathrm{BE}=$ benevolence, $\mathrm{UN}=$ universalism.

\section{Multilevel Regression Analysis}

A multi-level hierarchical regression analysis using the CLOP (James \& Williams, 2000) procedure was conducted. Predictors were entered in four different blocks. As in Study 2 (chapter three), socio-demographic variables which have previously been shown to be related to moral attitudes were entered first to control for them (Harding \& Phillips, 1986). These variables were gender, age, political orientation and religiosity. At Step 2, the individual's response set across all values was entered as a control variable (cf. Schwartz, 2007a). This variable consisted of the individual's mean ratings (MeanRATing) across all values for all three response scales (IMPortance, MORal, EXPectation). Including MeanRATing as a covariate in the regression model ensured that individual differences in the scale use were controlled for. Step 3 contained 
values at the individual-level: openness-to-change vs. conservation assessed with the IMPortance, MORal and EXPectation response scales. Hence, there were three predictors for values at the individual-level. Step 4 consisted of the following culturelevel variables: openness-to-change vs. conservation measured with the IMPortance, MORal and EXPectation response scale. Thus, there were three culture-level predictors.

Table 5.5 shows the correlations of all variables in the model. Some bivariate correlations were greater than .70 which may cause problems of multicollinearity when included in one and the same regression model (Tabachnick \& Fidell, 2007). Multicollinearity is the extent to which a variable can be explained by other variables included in the regression model (Hair, et al., 2006). It may lead to undesirable outcomes in the analysis such as affecting the statistical tests of the predictors or the overall model, incorrect estimates of predictors or even reversing the signs of the predictors. Running the full model as described above did show some of these statistical problems. For instance, the 'EXPectation' rating of openness-to-change vs. conservation values at the culture-level was negatively correlated with the outcome variable $(\mathrm{r}=-.27, \mathrm{p}<.001)$, but emerged as a positive predictor with a very high regression weight in the regression model $(\beta=.72, \mathrm{p}<.001)$. Multicollinearity can also be detected with more direct means, such as the 'tolerance' measure. Tolerance is defined as "the amount of variability of the selected independent variable not explained by the other independent variables" (Hair, et al., p. 227). There are no hard rules to determine multicollinearity, but a rule of thumb is that if the tolerance value is low (ranging between 0 and 1), it indicates a high degree of multicollinearity. For the present model, the tolerance value was fairly low for all three culture-level variables $(.13, .24, .24)$ indicating that multicollinearity may be a problem for the model. 
Table 5.5: Pearson correlations of predictors and criterion variable.

\begin{tabular}{|c|c|c|c|c|c|c|c|c|c|c|c|c|c|}
\hline & & 1 & 2 & 3 & 4 & 5 & 6 & 7 & 8 & 9 & 10 & 11 & 12 \\
\hline & $\begin{array}{l}\text { 1. MA } \\
\text { (outcome variable) }\end{array}$ & - & & & & & & & & & & & \\
\hline & 2. Gender & .03 & - & & & & & & & & & & \\
\hline & 3. Age & -.05 & $-.10^{\mathrm{b}}$ & - & & & & & & & & & \\
\hline & 4. Political orientation & $.22^{\mathrm{c}}$ & $-.09^{\mathrm{a}}$ & .03 & - & & & & & & & & \\
\hline & 5. Religiosity & $.17^{\mathrm{c}}$ & .00 & $-.14^{\mathrm{c}}$ & .05 & - & & & & & & & \\
\hline & 6. MRAT & $.24^{\mathrm{c}}$ & -.04 & $-.10^{b}$ & .04 & .05 & - & & & & & & \\
\hline \multirow[t]{3}{*}{$\begin{array}{l}\text { Individual- } \\
\text { level }\end{array}$} & 7. OPCH vs. CONS: IMP & $-.38^{\mathrm{c}}$ & -.03 & .06 & $-.23^{c}$ & $-.10^{b}$ & $-.21^{\mathrm{c}}$ & - & & & & & \\
\hline & 8. OPCH vs. CONS: EXP & $-.10^{\mathrm{b}}$ & -.06 & $.08^{\mathrm{a}}$ & .04 & $-.12^{b}$ & -.04 & $.13^{\mathrm{c}}$ & - & & & & \\
\hline & 9. OPCH vs. CONS: MOR & $-.22^{\mathrm{c}}$ & -.04 & .00 & $-.17^{\mathrm{c}}$ & $-.11^{b}$ & -.05 & $.44^{\mathrm{c}}$ & $.14^{\mathrm{c}}$ & - & & & \\
\hline \multirow[t]{3}{*}{$\begin{array}{l}\text { Culture- } \\
\text { level }\end{array}$} & 10. OPCH vs. CONS: EXP & $-.27^{\mathrm{c}}$ & $.07^{\mathrm{a}}$ & $.16^{\mathrm{c}}$ & -.01 & $-.14^{\mathrm{c}}$ & $-.30^{\mathrm{c}}$ & $.33^{\mathrm{c}}$ & $.30^{\mathrm{c}}$ & $.22^{\mathrm{c}}$ & - & & \\
\hline & 11. OPCH vs. CONS: IMP & $-.40^{\mathrm{c}}$ & $.07^{\mathrm{a}}$ & $.17^{\mathrm{c}}$ & $-.09^{\mathrm{a}}$ & -.03 & $-.33^{c}$ & $.38^{\mathrm{c}}$ & $.24^{\mathrm{c}}$ & $.17^{\mathrm{c}}$ & $.85^{\mathrm{c}}$ & - & \\
\hline & 12. OPCH vs. CONS: MOR & $-.32^{\mathrm{c}}$ & .03 & $.11^{\mathrm{b}}$ & -.01 & $-.21^{\mathrm{c}}$ & $-.22^{\mathrm{c}}$ & $.26^{\mathrm{c}}$ & $.26^{\mathrm{c}}$ & $.26^{\mathrm{c}}$ & $.85^{\mathrm{c}}$ & $.67^{\mathrm{c}}$ & - \\
\hline
\end{tabular}

Note. ${ }^{\mathrm{a}} p<.05 .{ }^{\mathrm{b}} p<.01,{ }^{\mathrm{c}} p<.001$

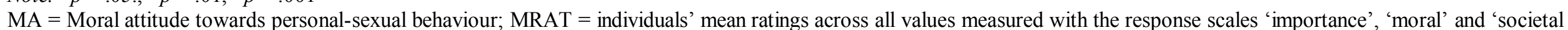

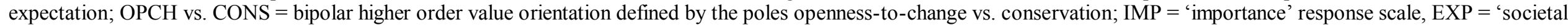
expectation response scale', MOR = 'moral' response scale. 
It appeared from the correlation table that the problem was mostly associated with the high intercorrelation between the culture-level variables EXPectation and MORal as well as EXPectation and IMPortance. Hence, two separate models were conducted, one with all individual-level predictors as described above, but only EXPectation as a culture-level predictor (Model 1) and another one with all the individual-level predictors and IMPortance as well as MORal as culture-level predictors (Model 2). The tolerance values for Model 1 were acceptable with a minimum of .74. The tolerance values for Model 2 were relatively low with .44 and .49 for the culture-level predictors. However, this model did not show the 'symptoms' of the previous full model, such as reversed signs or very high regression weights. Other multicollinearity indices were also acceptable, for instance, the maximum VIF (Variance Inflation Factor), which is the reciprocal of the tolerance and should not be greater than 10 , was here 2.25 . On the basis of these criteria, it was decided to retain IMPortance and MORal as culture-level predictors in Model 2.

Table 5.6 shows the final regression weights for Model 1. On the first step, demographic variables alone explained $8 \%$ of the variance, with conservative political orientation and religiosity making a significant contribution to the prediction of strict moral attitudes, corroborating findings of past research (Harding \& Phillips, 1986; Vauclair \& Fischer, 2009, see also chapter three). Age may not have emerged as a predictor of moral attitudes, since the sample consisted of students which is a relatively homogeneous age group. Gender may also not have been a predictor, because gender differences are possibly less pronounced in student samples than they are in more heterogeneous samples (see, for example, Study 2 of this thesis). Adding MeanRATing in the second step increased the explained variance to $13.1 \%$. This indicates that individuals with the tendency to score highly on their value ratings also scored highly on their moral attitude ratings, which may be a sign of individual-level acquiescence. 
The addition of individual-level values to the prediction of moral attitudes increased the explained variance to $21.5 \%$. The only significant predictor was the IMPortance rating of openness-to-change vs. conservation values, with endorsing conservative values (negative pole) being related to a more strict moral attitude which is consistent with what was expected. The final step consisted of the culture-level variable openness-tochange vs. conservation values rated as a societal expectation. The variable was a significant predictor and increased the explained variance to $22.7 \%$. This means that conservation values rated as a societal expectation in some cultural samples were related to individuals' strict stance on personal-sexual issues and vice versa for openness-to-change values.

Table 5.6: Summary of hierarchical regression analysis predicting individuals' strictness in their attitudes towards personal-sexual issues

(Survey Version 1, Model 1).

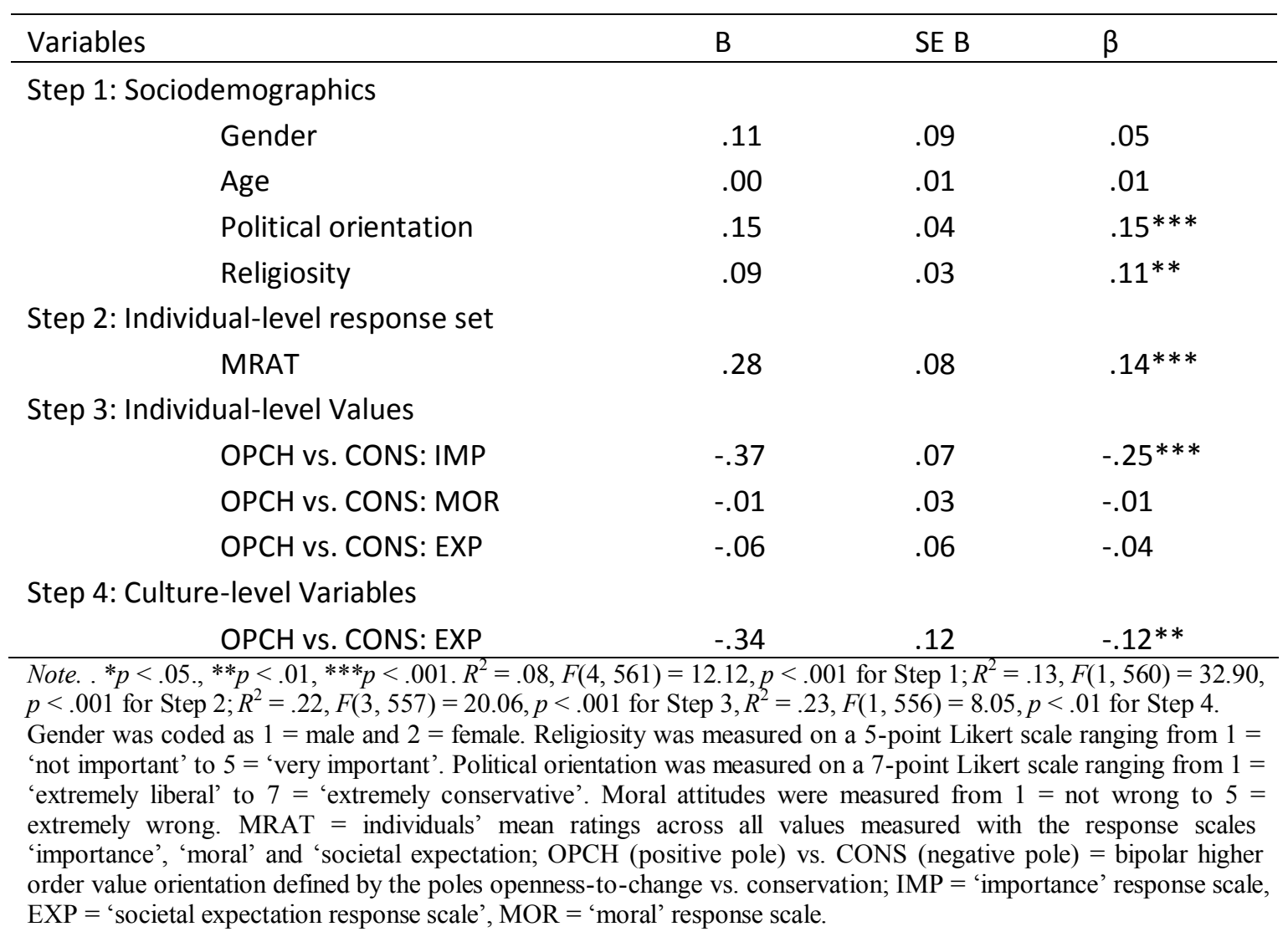


Model 2, which was based on all individual-level predictors from above, but contained IMPortance and MORal as culture-level predictors instead of EXPectation, yielded virtually the same results for Step 1, 2 and 3 of the previous model (see Table 5.7 for the final regression weights) explaining $21.5 \%$ of the variance in step 3 . At the culture-level, IMPortance ratings emerged as a significant cross-level predictor, whereas MORal was not significant. This final step increased the explained variance to $27.4 \%$.

Table 5.7: Summary of hierarchical regression analysis predicting individuals' strictness in their attitudes towards personal-sexual issues

(Survey Version 1, Model 2).

\begin{tabular}{|c|c|c|c|}
\hline Variables & $\mathrm{B}$ & SE B & $\beta$ \\
\hline \multicolumn{4}{|l|}{ Step 1: Sociodemographics } \\
\hline Gender & .14 & .09 & .06 \\
\hline Age & .01 & .01 & .03 \\
\hline Political orientation & .15 & .04 & $.15^{* * *}$ \\
\hline Religiosity & .10 & .03 & $.12^{* *}$ \\
\hline \multicolumn{4}{|l|}{ Step 2: Individual-level response set } \\
\hline MRAT & .20 & .08 & $.10 * * *$ \\
\hline \multicolumn{4}{|l|}{ Step 3: Individual-level Values } \\
\hline OPCH vs. CONS: IMP & -.29 & .07 & $-.20 * * *$ \\
\hline OPCH vs. CONS: MOR & .02 & .03 & .02 \\
\hline OPCH vs. CONS: EXP & -.05 & .06 & -.04 \\
\hline \multicolumn{4}{|l|}{ Step 4: Culture-level Variables } \\
\hline OPCH vs. CONS: IMP & -.90 & .21 & $-.23 * * *$ \\
\hline OPCH vs. CONS: MOR & -.40 & .30 & -.07 \\
\hline \multicolumn{4}{|c|}{$\begin{array}{l}\text { Note. }{ }^{*} p<.05 ., * * p<.01, * * * p<.001 . R^{2}=.08, F(4,561)=12.12, p<.001 \text { for Step } 1 ; R^{2}=.13, F(1, \\
560)=32.90, p<.001 \text { for Step } 2 ; R^{2}=.22, F(3,557)=20.06, p<.001 \text { for Step } 3, R^{2}=.26, F(2,555)= \\
22.45, p<.001 \text { for Step } 4 . \\
\text { Gender was coded as } 1=\text { male and } 2=\text { female. Religiosity was measured on a } 5 \text {-point Likert scale } \\
\text { ranging from } 1=\text { 'not important' to } 5=\text { 'very important'. Political orientation was measured on a 7-point } \\
\text { Likert scale ranging from } 1=\text { 'extremely liberal' to } 7=\text { 'extremely conservative'. Moral attitudes were } \\
\text { measured from } 1 \text { = not wrong to } 5=\text { extremely wrong. MRAT = individuals' mean ratings across all } \\
\text { values measured with the response scales 'importance', 'moral' and 'societal expectation; OPCH } \\
\text { (positive pole) vs. CONS (negative pole) = bipolar higher order value orientation defined by the poles } \\
\text { openness-to-change vs. conservation; IMP = 'importance' response scale, EXP = 'societal expectation } \\
\text { response scale', MOR = 'moral' response scale. }\end{array}$} \\
\hline
\end{tabular}


To conclude, after controlling for demographic variables, as well as response sets in value ratings and individual-level values, 'EXPectation' ratings of openness-tochange vs. conservation values at the culture-level showed the same effect in predicting individuals' moral attitudes as 'IMPortance' ratings. Interestingly, 'EXPectation' ratings were not a predictor at the individual-level.

\section{Follow-up Analyses: Individual-level Mediation Model}

The correlations in Table 5.5. show that EXPectation and MORal ratings of openness-to-change versus conservation values at the individual-level were related to the outcome variable, however they did not account for unique variance in the outcome variable over and above IMPortance ratings. The non-significant result for EXPectation and MORal ratings at the individual-level may be due to a mediation effect of IMPortance ratings. Testing explicitly the interrelationship between the three variables EXPectation, IMPortance and the dependent variable (DV), as well as MORal, IMPortance and the DV, can provide some insight in why people hold certain attitudes and what the mechanism is that explains it. In the present case, it may be that moral attitudes can be explained with the internalization of values assessed through personally important values. This would be consistent with Ryan and Deci's (2000) SelfDetermination Theory which posits a progressive transformation of external values into personally important values. Hence, a mediation model was conducted as a follow-up analysis. Two mediation models were run using MedGraph (Jose, 2003). Figure 5.7 presents the results for the mediation analyses. 

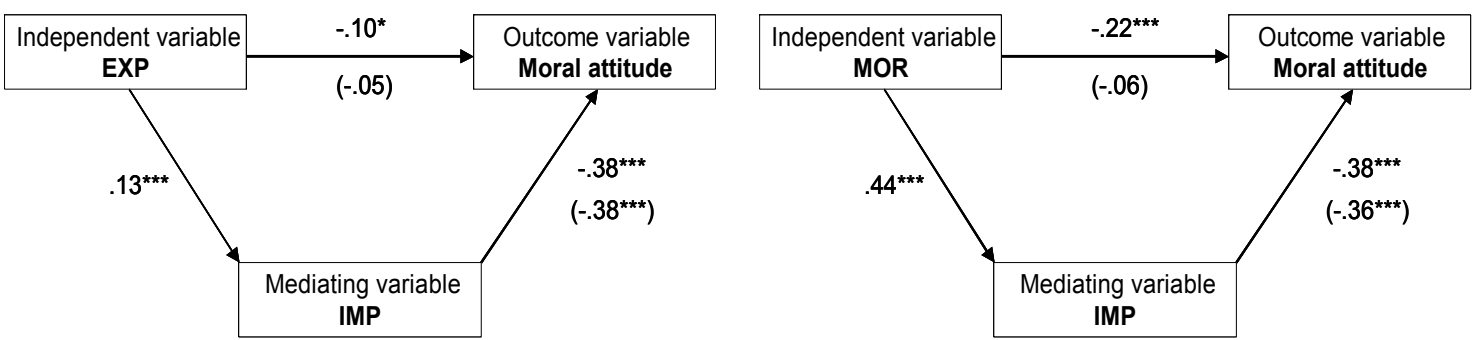

Figure 5.7: Two mediation models for different value ratings at the individual-level.

Note. Figures in parentheses denote the indirect effect.

Baron and Kenny's (1986) method was used to test for mediation which consists in three conditions. Starting with EXPectation ratings of openness-to-change versus conservation, they predicted moral attitudes, $\beta=-.10, p<.05$, fulfilling the first condition specified by Baron and Kenny (1986). EXPectation ratings also significantly predicted IMPortance ratings, $\beta=.13, p<.001$, fulfilling the second condition. When moral attitudes were finally regressed simultaneously from EXPectation ratings and IMPortance ratings, the regression weight for IMPortance ratings was significant, $\beta=$ $.38, p<.001$, while the regression weight for EXPectation ratings became nonsignificant, $\beta=-.05, p>.05$. The Sobel test showed a significant drop in the reduction of the effect of EXPectation, $z=-3.12, p<.001$, indicating a full mediation. MORal ratings as the independent variable showed virtually the same effect, although the regression weights were in general somewhat higher than for the EXPectation rating model. To conclude, it appears that personal values (IMPortance) intervene between societal expectations (EXPectation) and moral attitudes as well as between personal moral values (MORal) and moral attitudes. 


\section{Cross-validation}

The main findings from above were cross-validated with the second version of the survey answered by an independent sample and using somewhat different values (see Table 5.2).

\section{MDS on Value Hierarchies}

Similarly to the analysis of the first version of the survey, a MDS was first performed on the pan-cultural value hierarchies produced by the different response scales. As can be seen in Figure 5.3 the two versions produced virtually the same result: Personal values (IMPortance rating scale) and societal moral values (EXPectation rating scale) were located opposite to each other. MORal ratings are located in between societal expectations and personal preferences.

Next, a MDS on the cultural value hierarchies produced by the five different response scales was performed. In order to statistically compare the similarity of results of the two survey versions, the coordinates of the elements in the two-dimensional space were correlated with each other. This was done after rotating the two solutions to maximal similarity with GPA. Both dimensions correlated very highly with each other. Product-moment correlation coefficients for dimension 1 and 2 were $.98(p<.001)$ and $.85(p<.001)$. Spearman correlations were very similar to Pearson correlation coefficients $\left(r_{s \operatorname{dim} 1}(39)=.98, p<.001 ; r_{s \operatorname{dim} 2}(39)=.87, p<.001\right)$.

This validates two conclusions drawn from the analysis of the first survey version. First, values measured as societal expectations and personal preferences yield different value hierarchies, whereas personal moral values are somewhat similar to both of them. Secondly, cultural samples are ordered along the hypothetical individualism/ collectivism dimension in regard to their value hierarchies no matter what kind of values are assessed. 
Regression Analyses

Two multilevel regression analyses using the CLOP method were run on the data of the second version of the survey. The two models were again identical except for the culture-level predictors. Model 1 contained EXPectation ratings of openness-tochange vs. conservation values as culture-level variables and Model 2 contained IMPortance and MORal ratings of these values. The results showed the same pattern of significance as for Version 1 of the survey (see Table 5.8 and 5.9): A conservative political orientation, religiosity, acquiescence response set and the personal importance of conservation values at the individual-level were related to a stricter attitude towards personal-sexual issues. At the culture-level, the perceived societal expectation (Model 1) and the personal importance of conservation values (Model 2) predicted stricter moral judgments. 
Table 5.8: Summary of cross-validating hierarchical regression model predicting individuals' strictness in their attitudes towards personal-sexual issues (Survey Version 2, Model 1).

\begin{tabular}{|c|c|c|c|}
\hline Variables & $\mathrm{B}$ & SE B & $\beta$ \\
\hline \multicolumn{4}{|l|}{ Step 1: Sociodemographics } \\
\hline Gender & .10 & .08 & .04 \\
\hline Age & .01 & .01 & .06 \\
\hline Political orientation & .12 & .03 & $.12 * * *$ \\
\hline Religiosity & .08 & .02 & $.12 * * *$ \\
\hline \multicolumn{4}{|l|}{ Step 2: Individual-level response set } \\
\hline MRAT & .25 & .07 & $.13 * * *$ \\
\hline \multicolumn{4}{|l|}{ Step 3: Individual-level Values } \\
\hline OPCH vs. CONS: IMP & -.45 & .05 & $-.35 * * *$ \\
\hline OPCH vs. CONS: MOR & -.06 & .04 & -.05 \\
\hline OPCH vs. CONS: EXP & .01 & .03 & .02 \\
\hline \multicolumn{4}{|l|}{ Step 4: Culture-level Variables } \\
\hline OPCH vs. CONS: EXP & -.39 & .11 & $-.13 * *$ \\
\hline \multicolumn{4}{|c|}{$\begin{array}{l}\text { Note. }{ }^{*} p<.05, * * p<.01,{ }^{* * *} p<.001 . R^{2}=.11, F(4,670)=20.50, p<.001 \text { for Step } 1 ; R^{2}=.20, F(1, \\
669)=78.53, p<.001 \text { for Step } 2 ; R^{2}=.33, F(3,666)=42.10, p<.001, R^{2}=.34, F(1,665)=12.51, p< \\
.001 \text { for Step } 4 . \\
\text { Gender was coded as } 1=\text { male and } 2=\text { female. Religiosity was measured on a 5-point Likert scale } \\
\text { ranging from } 1=\text { 'not important' to } 5=\text { 'very important'. Political orientation was measured on a 7-point } \\
\text { Likert scale ranging from } 1=\text { 'extremely liberal' to } 7=\text { 'extremely conservative'. Moral attitudes were } \\
\text { measured from } 1=\text { not wrong to } 5=\text { extremely wrong. MRAT = individuals' mean ratings across all } \\
\text { values measured with the response scales 'importance', 'moral' and 'societal expectation; OPCH } \\
\text { (positive pole) vs. CONS (negative pole) = bipolar higher order value orientation defined by the poles } \\
\text { openness-to-change vs. conservation; IMP = 'importance' response scale, EXP = 'societal expectation } \\
\text { response scale', MOR = 'moral' response scale. }\end{array}$} \\
\hline
\end{tabular}


Table 5.9: Summary of cross-validating hierarchical regression model predicting

individuals' strictness in their attitudes towards personal-sexual issues (Survey Version

2, Model 2).

\begin{tabular}{|c|c|c|c|}
\hline Variables & $\mathrm{B}$ & SE B & $\beta$ \\
\hline \multicolumn{4}{|l|}{ Step 1: Sociodemographics } \\
\hline Gender & .10 & .08 & .04 \\
\hline Age & .01 & .01 & .06 \\
\hline Political orientation & .12 & .03 & $.12 * * *$ \\
\hline Religiosity & .06 & .02 & $.09 * *$ \\
\hline \multicolumn{4}{|l|}{ Step 2: Individual-level response set } \\
\hline MRAT & .15 & .07 & $.08^{*}$ \\
\hline \multicolumn{4}{|l|}{ Step 3: Individual-level Values } \\
\hline OPCH vs. CONS: IMP & -.38 & .06 & $-.30 * * *$ \\
\hline OPCH vs. CONS: MOR & -.06 & .05 & -.06 \\
\hline OPCH vs. CONS: EXP & .02 & .02 & .02 \\
\hline \multicolumn{4}{|l|}{ Step 4: Culture-level Variables } \\
\hline OPCH vs. CONS: IMP & -.69 & .15 & $-.26 * * *$ \\
\hline OPCH vs. CONS: MOR & .10 & .16 & .03 \\
\hline \multicolumn{4}{|c|}{$\begin{array}{l}\text { Note. }{ }^{*} p<.05,{ }^{*} p<.01,{ }^{* *} p<.001 . R^{2}=.11, F(4,670)=20.50, p<.001 \text { for Step } 1 ; R^{2}=.20, F(1, \\
669)=78.53, p<.001 \text { for Step } 2 ; R^{2}=.33, F(3,666)=42.10, p<.001, R^{2}=.37, F(2,664)=18.77, p< \\
.001 \text { for Step } 4 . \\
\text { Gender was coded as } 1=\text { male and } 2=\text { female. Religiosity was measured on a 5-point Likert scale } \\
\text { ranging from } 1=\text { 'not important' to } 5=\text { 'very important'. Political orientation was measured on a 7-point } \\
\text { Likert scale ranging from } 1=\text { 'extremely liberal' to } 7=\text { 'extremely conservative'. Moral attitudes were } \\
\text { measured from } 1=\text { not wrong to } 5=\text { extremely wrong. MRAT = individuals' mean ratings across all } \\
\text { values measured with the response scales 'importance', 'moral' and 'societal expectation; OPCH } \\
\text { (positive pole) vs. CONS (negative pole) = bipolar higher order value orientation defined by the poles } \\
\text { openness-to-change vs. conservation; IMP = 'importance' response scale, EXP = 'societal expectation } \\
\text { response scale', MOR = 'moral' response scale. }\end{array}$} \\
\hline
\end{tabular}

Individual-level Mediation Model

Two mediation models were again conducted as a follow-up analyses using MedGraph (Jose, 2003). The results for the mediating effect of personal values (IMP) on personal moral values (MOR) and moral attitudes were virtually the same as in the analysis for Version 1 of the survey. MORal ratings predicted moral attitudes, $\beta=-.33$, $p<.001$ and also significantly predicted IMPortance ratings, $\beta=.54, p<.001$. When moral attitudes were regressed simultaneously from MORal ratings and IMPortance ratings, the regression weight for IMPortance ratings was significant, $\beta=-.33, p<.001$, while the regression weight for MORal ratings became non-significant, $\beta=-.07, p>$ 
.05. The Sobel test showed a significant drop in the reduction of the effect of MORal, $z$ $=-10.63, p<.001$, indicating a full mediation. The result looked very different for EXPectation ratings. EXPectation ratings were not significantly correlated with moral attitudes $(r=-.01, p>.05)$ meaning that the first condition for pursuing a mediation model as specified by Baron and Kenny (1986) was not fulfilled ${ }^{22}$.

To conclude, the mediating effect of IMPortance ratings on the relationship between MORal ratings and moral attitudes was crossvalidated. However, no mediation model could be established for EXPectation ratings and moral attitudes, since there was no significant relationship between the two variables. Hence, it seems that perceived societal moral values are not reliably related to moral attitudes at the individual-level.

\section{General Discussion}

Study 3 confirmed that openness-to-change vs. conservation values at the culture-level are related to moral attitudes towards personal-sexual behaviours if rated as (1) personal values (IMPortance), (2) personal moral values (MORal), and

societal moral values (EXPectation). Due to multicollinearity problems between IMPortance and EXPectation ratings, it was not possible to test the incremental validity of openness-to-change rated as societal expectations over and above these values rated

\footnotetext{
${ }^{22}$ More recently, Preacher and Hayes (2004) suggested that this condition does not need to be fulfilled in order to test a mediation model. They distinguish between mediated effects and indirect effects. A mediated effect is a special case of indirect effects when there is only one mediating variable and a significant relationship between the IV and DV in the first place. There is no such assumption in the assessment of indirect effects. It is possible to find a significant indirect effect even when the IV and DV are not significantly correlated. The Sobel test (Sobel, 1982) provides a test of an indirect effect by comparing the strength of the indirect effect of the IV on the DV to the null hypothesis that it equals zero. Preacher and Hayes (2004) provide a macro for SPSS that allows testing the significance of the indirect effect both parametrically and non-parametrically (in case of non-normal distributions). Using the macro with the present data showed that the Sobel test was not significant $(z=-.19, p>.05)$, therefore suggesting no mediation. The non-parametric test which consists of bootstrapped estimates of the indirect effect (here for 1,000 bootstrap iterations) also indicated a non-significant finding. The bias corrected and accelerated bootstrapped $95 \%$ confidence interval indicated that the true indirect effect was estimated to lie between -0.04 and 0.03 . Hence, it can be concluded that the mediation model was not replicable with Version 2 of the survey.
} 
personally important. Nevertheless, EXPectation ratings at the culture-level performed as well as IMPortance ratings in the prediction of moral attitudes after controlling for demographics, individuals' response set in rating value items, and individual-level value ratings (IMPortance, MORal, and EXPectation). Personal moral values at the culture-level were significantly correlated with the outcome variable, but did not add incremental validity over and above the importance ratings.

Although the incremental validity of EXPectation value ratings at the culturelevel could not be tested (or could not be confirmed in the case of MORal ratings respectively) it is an important finding that EXPectation value ratings performed as well as IMPortance ratings at the culture-level in the prediction of moral attitudes. It has been pointed out in the literature that there is the problem of causal inconsistency if micro- and macro-level constructs are not operationalized differently (Liska, 1990). This is an important issue if the research focus is, for example, on value change with the underlying assumption that cultural values (macro-level variables) influence individuals' values (micro-level variable). It is hard to comprehend how a change in cultural values can cause a change in individual values if the change in cultural values is measured through the aggregated changes in individual values (Roe \& Ester, 1999). This methodological flaw can be avoided if macro-level constructs are measured independently from micro-level constructs (Liska, 1990). EXPectation ratings of values at the culture-level fulfil conceptually and methodologically the requirements of a valid macro-level construct: the EXPectation rating scale measures conceptually the perceived 'cultural press' and I showed that it significantly predicted the criterion variable corroborating established theories on the cultural variability of morality. Future research is needed in order to investigate whether EXPectation value ratings as a macrolevel variable predict other criterion variables than attitudes towards personal-sexual behaviours. 
Furthermore, value ratings at the individual-level showed that IMPortance ratings were relatively strong predictors for moral attitudes and that EXPectation and MORal did not add unique variance. Follow-up analysis testing whether personal values (IMP) were a mediator for the relationship between societal moral values (EXP) and moral attitudes as well as personal moral values (MOR) and moral attitudes showed that full mediation occurred only reliably in two different samples in the case of personal moral values. This leads to two conclusions: (1) values rated as societal expectations seem to be a 'truly' culture-level construct, (2) internalization mechanisms, here the rating of values as personally important, may explain the overriding of personal moral values as a predictor for moral attitudes. Endorsing a value as personally important may be one step further in the internalization process than acknowledging a value as morally relevant. This would also be in line with Self-Determination Theory that proposes how extrinsic values and behaviours can be gradually internalized and become part of the self (Ryan \& Deci, 2000). The theory specifies that internal rewards and punishments come before endorsing a value or behaviour as personally important. Internal rewards and punishments may be regarded as conceptually related to personal moral values arousing feelings of remorse if they are violated; whereas personal importance ratings assess personal values which implies a conscious valuing. This theory would also suggest that highly internalized values are more strongly associated with other relevant variables such as moral attitudes, since they become part of a person's identity. This is what may have been found in the mediation analysis: personally important values override personal moral values in the prediction of individuals' moral attitudes.

This study also showed that students from very different cultures, differentiated a priori into collectivistic and individualistic samples, commonly regarded benevolence, universalism and conformity values as personal moral values. These three value types 
correspond to the three functions of morality proposed by Helkama (2004): benevolence relates to prosocial action, universalism to moral problem-solving (issues of justice), and conformity to the prevention of antisocial behaviour. These values may provide the internalized motivational basis for cooperation and mutually supportive social relations which is a basic requirement for every society in order to function smoothly (cf. Krebs, 2008). The findings are also consistent with research on qualities people value in their children: Baer, Curtis, Grabb and Johnston (1996) showed that there was great similarity across individuals from 15 countries with the most often mentioned qualities being honesty, tolerance and respect for other people, good manners, and the feeling of responsibility. These values can be assigned to the value types benevolence, universalism, and conformity respectively.

The findings for societal moral values provided a different picture. There was a clear partition between individualistic and collectivistic samples in regard to their societal moral value hierarchy (see MDS, Figure 5.4). This partition remained even after taking into account that response sets may enhance the differences between the two cultural groups (see MDS, Appendix E). The individualistic samples perceived achievement, self-direction and benevolence values as the most predominant societal expectations. In contrast, the collectivistic samples perceived conformity, security, and benevolence as prevalent societal expectations. Except for benevolence, the two cultural groups differed by perceiving values with either a personal (individualistic samples) or social focus (collectivistic samples) as societal expectations. This is consistent with theories on collectivism and individualism suggesting that in individualistic cultures, the basic unit of society is the individual with his/her attitudes, values, skills and goals, whereas in collectivistic cultures, the basic unit of society is the group (e.g. the family) to which people are expected to be very loyal and to be conforming to group norms (Hofstede, 1980; Kağıtçıbaşı, 1997; Triandis, 1995). Nevertheless, my finding deviates 
somewhat from theories on the cultural variability of morality. Shweder, et al. (Miller, et al., 1990; Shweder, et al., 1997) proposed that cultures differ in their emphasis on the ethics of Autonomy or Community. Furthermore, Graham et al. (2009) suggested that cultures differ in the sources of their moral intuitions which can be distinguished into a individualizing and binding foundation. Both theories parallel the individualism/ collectivism distinction in cross-cultural psychology. While the findings for the collectivistic samples are in line with the ethic of Community and the binding foundation of moral intuition, the findings for the individualistic samples are less evident. Although, Self-direction with its focus on a person's self-determination and autonomy is related to a rights-based morality, and hence in line with the two moral theories, the value of achievement is less so.

Graham et al. (2009) noted that Schwartz' value type of achievement does not fit into their own established moral taxonomies, nor the one Shweder et al. (1997) proposed. They suggested that achievement may point to an additional psychological mechanism that supports moral systems. Both self-direction and achievement are values with a personal focus serving individuals' interests (Schwartz, 1992). It is interesting that individuals from individualistic-oriented countries perceived these values as predominant expectations in their societies. One would think that societies would want their members to follow values with a social focus, so that social interactions are smooth and social life is organized. For example, Benevolence fits into this assumption: it is a value with a social focus and emerged as a high ranking perceived societal expectation in both individualistic and collectivistic samples.

How can the personal focus of societal expectations be explained in individualistic cultures? Moghaddam, Slocum, Finkel, Tzili, and Harré (2000) have pointed out that individuals from individualistic cultures may endorse duties, but interpret them in relation to the self and only indirectly to the community. For instance, 
young Americans completed the sentence 'My most important duty is . . ' to 'do well at school' or to 'fulfill my potential' etc. Hence, they focused more on a personal duty than on duties in relation to community. The value of achievement fits into this reasoning and would indicate that people from individualistic-oriented cultures also experience a sense of duty, but it is directed towards themselves and not others, as it is the case in collectivistic-oriented cultures. This would mean that a duty-based morality in Western cultures is also prevalent besides a rights-based morality. The fundamental difference in the Western duty-based morality is that its content refers to the individuals themselves in terms of an 'individual responsibility', a belief in 'self-help' and 'getting ahead personally' (see also Moghaddam, et al., 2000) which is very different to the duties in relation to community which are emphasized in collectivistic-oriented cultures.

The present study also showed that asking individuals to rate values as good vs. bad or right vs. wrong yielded very similar results in these samples (see, for example, MDS on response scales, Figure 5.3). This is somewhat contrary to what Wierzbicka (2006) has pointed out. She suggested that right and wrong possesses a unique meaning in the English language and only the evaluation terms good versus bad are recognized universally. In the present study, there were no major differences between the results produced by these two response scales. Contrary to my expectations, asking individuals to rate values as moral yielded meaningful results. Despite the term being relatively ambiguous, it appears that laypeople intuitively associate a certain set of values with morality. It is also very meaningful that findings of the MORal ratings were consistently located in-between personally important values and societal expectation (see, for example, MDS Figure 5.3 and 5.4). There appears to be an underlying continuum reflecting different degrees of internalization of values ranging from completely extrinsic (societal expectations) to somewhat extrinsic (personal moral values) and completely intrinsic (personal values). 
Last but not least, for cross-cultural research, it is noteworthy that the most clear partition into individualistic and collectivistic samples was found with values rated as societal expectations and as personal values (see MDS, Figure, 5.5) even after taking into account response sets (see Appendix E).

\section{Limitations}

There are a number of limitations to consider when evaluating the findings of this study. First, by recruiting university students, there remains the question whether the results are generalizable to the wider cultural population from which the students were sampled. It may be, for instance, that achievement was regarded as a societal expectation in the individualistic sample, because we are dealing with student populations for which success may be more salient than for other samples. Nevertheless, it is interesting to note, that the matched collectivistic sample also faces the pressure of being successful in regard to academic requirements, but did not rate it as a predominant societal expectation. Furthermore, the frame of reference was deliberately chosen as referring to the wider society assessing the 'cultural press' that applies to everyone in society. Future research will need to examine whether the personal focus of individualistic samples in regard to perceived societal expectations remains if nonstudent samples, for example employees, are surveyed.

A further limitation concerns the data from the U.K. Data were here collected online and hence differed in the administration format compared to the other samples. There is an ongoing debate in the literature whether online responses differ from paper and pencil responses. Recently, Meade, Michels and Lautenschlager (2007) showed that scores in personality scales collected via the internet and via paper and pencil were comparable with one another. It emerged as more important to either allow all respondents to choose the administration format or to impose it on participants. Since in 
the present study students were not given a choice between administration formats, there was no inconsistency in this regard. Furthermore, responses of the U.K. sample showed the expected association with other individualistic and collectivistic samples. In regard to the sample from the U.K., it is also noteworthy that it was relatively small. Nevertheless, in analyses involving country-level data, there is a substantial benefit from adding further data points at the higher level, even if some samples do not contain many cases (Smith, et al., 2002).

Another point to consider is that sample characteristics differed in regard to the percentages of females, average age and also religion and economic development of the country. Age and gender differences as well as religiosity were taken into account in the hierarchical regression models by controlling for demographics in the first step. In regard to the value ratings and their analysis through multidimensional scaling, differences in these individual- and country-level variables may have confounded with cultural differences. There are several reasons which render this very unlikely. First, past research has not yielded consistent evidence for the existence of gender differences in morality (Walker, 1991). Secondly, the age differences across samples were not very big. All samples were, in average, above 20 and below 27 years old. It would have been more problematic if some cultural samples were mainly adolescents, who still are in the process of defining their identity, which may have influenced what is regarded as personally important to them or morally relevant. Third, the observed differences between the cultural samples corroborated what was expected in terms of a partition into individualistic and collectivistic samples. Hence, age, gender, religion and also economic development can be largely ruled out as rival explanations for the findings.

It is also noteworthy that, although culture-level values added significant explained variance in the CLOP regression analysis, the magnitude of this variance was not very big, adding only $1.2 \%$ unique variance, for example, in Model 1 (Survey 
Version 1). This can be explained to some extent with the characteristics of CLOP analyses which uses both individual-level and unit-level independent variables to explain total variance in the dependent variable. Contrary to HLM, CLOP analysis does not partition into within- and between-unit components leading to the effect that CLOP reports effect sizes much smaller than in HLM (Klein, et al., 2000). Apart from that, it is a common finding that contextual variables often explain little variance in individuallevel dependent variables and some researchers have used this as an argument to remain on the individual-level only (Liska, 1990). However, as Liska (1990) puts it "even if contextual effects are small, they are theoretically significant because they operate over a wide range of research areas and dependent variables" (p. 299). Future research could examine the effect of societal expectations at the culture-level on other dependent variables than moral attitudes.

Another important point to consider is the fact that I used the same value types at the individual- and culture-level. Schwartz $(1994,2006)$ clearly indicated that the individual-level value structure is different from the culture-level value structure. Taking individuals" values to describe cultures means committing the "reverse ecological fallacy" (Hofstede, 1980, p. 29) which, it could be argued, I have done here. Nevertheless, equating the individual-level value types with culture-level values has already been done sporadically in the cross-cultural literature. For instance, Kağıtçıbaşı (1997) theoretically compared Schwartz' value orientation openness-to-change versus conservation with Hofstede's culture-level dimensions individualism versus collectivism. Lönnqvist et al. (2009) performed a group-level analysis to relate values to self-esteem in 14 cultural samples. They used Schwartz' ten individual-level value types for this purpose and not the seven culture-level values. In a similar vein, Verkasalo et al. (2009) used Schwartz' higher order value orientations at the individual-level to investigate cross-cultural differences across 25 European countries. 
A last point to consider concerns the value survey. Since moral values are a multifaceted concept which may be evaluated in different ways (such as something good as opposed to bad or right as opposed to wrong), a number of response scales have been included in this survey. Each respondent rated each value on five different response scales. This task is certainly cognitively demanding, and to some extent, tedious. It may also invite mistakes if the direction of the bipolar response scales is changed to counteract response sets. The scale may be suitable for university students but would need to be simplified for other samples such as children or uneducated adults. A possibility would be to take the Portrait Value Survey, which has been developed for the purpose of assessing values in a simpler way, and to add response scales that tap into the moral evaluation of values. It would not be necessary to provide five different response scales, since my research has shown that the evaluation of right/wrong, $\mathrm{good} / \mathrm{bad}$ is largely equivalent to moral/immoral.

\section{Future Prospects}

This study yielded a number of new findings. Two of them are especially worthwhile to pursue in future research. First, the perception of prescriptive values imposed from society (values as societal expectations) shows a social focus in collectivistic samples and a personal focus in individualistic ones. This finding does only partially fit into previous theories of morality (Haidt \& Graham, 2006; Haidt \& Joseph, 2004, for Moral Foundation Theory; Shweder, et al., 1997, for the three ethics of moral discourse). The finding that achievement is perceived as a very salient societal expectation in individualistic-oriented societies is surprising and worth further research since it points to an additional psychological system overlooked so far that may contribute to moral judgments (see Graham, et al., 2009). 
Secondly, it has been emphasized that potential effects of response bias should be acknowledged and addressed in data analysis involving cross-cultural comparisons and unpackaging studies (Matsumoto \& Yoo, 2006; Smith, 2005). This study has shown that cultural samples showed a virtually perfect partition into collectivistic and individualistic samples when response sets were not controlled for (see MDS, Figure 5.4). This is an interesting finding since other studies found the opposite, i.e. only after controlling for response sets, the predicted contrast between individualistic and collectivistic samples emerged (e.g. Schimmack, Oishi, \& Diener, 2005). The problem with removing response sets is that true differences may also be removed from the data. It is worthwhile to examine this bias itself, because it may reveal useful information about a culture's influence on psychological processes (Bond, 2002) or communication style affecting how people respond to questionnaires (Smith, 2004). Future studies may uncover the function and effect of certain response sets associated with culture. More systematic research in this area would reveal important insights into values research and the role of response sets, as something that needs to be controlled for or that can be examined as an expression of culture. 


\section{An Implicit Measure of Moral Values: \\ Lay People's Associations of the Moral Person}

\section{Introduction}

In chapter two of this thesis, I pointed out that cultural values can be conceptualized as general guidelines that regulate individuals' behaviour so that a more organized collective life is achieved. New members are explicitly and implicitly taught what is appropriate or socially desirable in a particular culture. Conventional approaches in deriving cultural values (e.g. Schwartz, 1992) do not directly assess this social component of values. Instead they are based on the assumption that what is good and socially desirable (the cultural ideals respectively) can be inferred from aggregated data on what is personally important (cf. Schwartz, 2006b). However, whether this inference is justified is not only a conceptual question, but also an empirical one.

Throughout the present thesis it has been emphasized that one of culture's functions is to regulate collective life by imposing constraints on what is acceptable and what is not. These binding normative expectations reflect a conventional kind of morality. Hence, the study of morality, and more particularly of moral values, may add an important insight into the cultural aspect of values. Similarly to Study 3 in this thesis, this chapter is targeted at investigating the moral component of values. What is entirely different is the approach that is taken here: instead of measuring values explicitly by asking for their moral relevance, the aim is to uncover moral values of different cultural groups with the help of an implicit method, i.e. through laypeople's associations of the concept of a moral person. 
In other words, the aim is to uncover what kind of attributes come into laypersons' mind when thinking of a 'moral person'. Hence, the methodological approach taken here is prototype analysis (see e.g. Fehr \& Russell, 1984; Rosch, 1975; Walker \& Pitts, 1998b).

Rosch (1975), who developed Prototype Theory, argued that many everyday concepts, such as fruits or tools, lack defining attributes. Instead they are organized around examples or exemplars which are referred to as a prototype. Rosch (1975) found, for instance, that exemplars of the prototype fruit were plum, lemon, cranberry, and coconut. Prototype analysis attempts to detect the exemplars of a prototype by examining the associations that a group of people has in regard to a concept's meaning. Since a prototype is usually based on group-generated exemplars, it describes the normative associative meaning of a term. It reflects a form of "collective wisdom" (Horowitz \& Turan, 2008, p. 1059) and is therefore especially appropriate for this study's cross-cultural focus.

In the case of a moral prototype, an exemplar would also mean a conception of attributes that an ideal person should have in order to qualify as a moral person (Walker \& Pitts, 1998b). Recent research has pointed out that there is an important linkage between exemplars and the study of culture. Fu and Chiu (2007), for example, stated that exemplary persons are "public carriers of culture as well as vehicles of intergenerational transmission of cultural values" (p. 650). Hence, detecting the associations with a 'moral person' in different cultures should provide an important insight into what it means to be 'moral' in a particular culture, and therefore into the most precious cultural values.

In the next section, the rationale of taking an implicit approach to moral values and the study of culture is outlined in more detail. 


\section{Why Implicit?}

It is widely acknowledged that cultural knowledge consists not only of explicit, but also of implicit knowledge (e.g. D'Andrade, 1984; Kroeber \& Kluckhohn, 1952). Triandis (1995), for instance, pointed out that one of culture's most important aspects is its 'unstated assumptions'. They are so fundamental that we are often unaware of them. This may equally apply to the area of morality and cultural variations defining what it means to be a good or a moral person respectively. Markus and Hamedani (2007), for example, referred to this very issue when saying that the "exposure to meanings and practices prevalent in a given context can provide a network of implicit and explicit associations to guide what it means to be a good person and competent social actor in that context" (pp. 23, italics added).

Not only cross-cultural psychologists have referred to cultural knowledge as consisting of implicit and explicit associations, but researchers in the area of morality have also recently emphasized the importance of implicit processes in moral functioning (Haidt, 2001; Haidt \& Joseph, 2004; Haidt, et al., 1993). Haidt and colleagues (e.g. Haidt, et al., 1993) found that moral judgments of right and wrong consisted of intuitive, reflexive and automatic mechanisms rather than of explicit moral reasoning. The request to explicitly articulate why an issue had been judged as right or wrong commonly led to a phenomenon that Haidt called 'moral dumbfounding' (see Haidt, 2001). Moral dumbfounding occurs when individuals stutter, laugh and express surprise at their inability to find reasons for their moral judgments.

Since the term implicit is widely used, but rarely defined (De Houwer \& TeigeMocigemba, 2009), and even within cross-cultural psychology it may be understood in different ways - for example, explicit as referring to physical objects of culture and implicit as referring to the psychological aspect of culture (cf. Matsumoto, 2007) - I shall briefly outline how it is understood in the scope of this thesis. When referring to 
explicit versus implicit knowledge assessment, what is meant here is the contrast between assessing a psychological construct by asking individuals directly about it (e.g. through self-reports) versus assessing the construct indirectly by inferring it from a person's automatic responses (cf. De Houwer \& Teige-Mocigemba, 2009). An automatic response is one that lacks awareness, intention, efficiency and control (Uleman, Saribay, \& Gonzalez, 2008). Associations have been commonly regarded as automatic responses (De Houwer \& Teige-Mocigemba, 2009; Greenwald \& Banaji, 1995). Furthermore, Smith, et al. (2007) pointed out that feature-listing instructions used in prototype analyses evoke individuals' implicit cognition, since there are no external cues as to the specific content of the prototype.

The benefits of taking an implicit approach to moral values are threefold: First, it is in line with the theoretical propositions outlined above that culture and morality consist not only of explicit, but also of implicit knowledge and processes. Secondly, findings from an implicit measure on moral values that converge with an explicit measure, as employed in Study 3 of this thesis, would corroborate the validity of measuring moral values. This would be a multi-method validation according to Campbell and Fiske (1959). Third, an open-ended approach that detects moral values in different cultures provides an opportunity to identify new moral values that are not contained in expert-designed explicit self-reports on values. A number of researchers have pointed out the advantage of taking a naturalistic approach to morality (Smith, Türk Smith, \& Christopher, 2007; Walker \& Pitts, 1998b). By relying on laypeople's everyday understanding of morality it is possible to understand how morality is construed in everyday life. This may provide a new perspective on the construct of moral values beyond the predefined value categories used in Study 3.

Hence, the present study was designed to tackle the implicit cultural knowledge of moral values. In the following sections, past research focusing on morality and 
culture using prototype analyses is briefly summarized. The research questions of the present study are embedded in the context of this research.

\section{Prototype Analyses}

One of the general principles of knowledge activation is accessibility. Accessibility can be defined as the activation potential of available knowledge (Higgins, 2000). The activation of knowledge structures can be studied through a prototype analysis (cf. Smith, et al., 2007). A prototype of a concept is "an ordered list of features that informants judge to be the most important associates for describing some of the concept's associative meaning" (Horowitz \& Turan, 2008, p. 1057). When prototypes are studied, researchers typically rely on two samples of informants: the 'nominators' who generate features of the prototype through free-listings, and the 'raters' who rate the importance of each feature in terms of how well it defines the prototype in question (Horowitz \& Turan, 2008). From these importance ratings it can be derived which features are more central or more peripheral in their association with the prototype (for practical examples see e.g. Fehr, 1988; Fehr \& Russell, 1984; Kearns \& Fincham, 2004; Lapsley \& Lasky, 2001; Walker \& Pitts, 1998b). However, more recently prototypes have also been inferred from the first step alone, i.e. from the free-listed features produced by nominators (Gregg, Hart, Sedikides, \& Kumashiro, 2008; Smith, et al., 2007). The underlying assumption hereby is that the group-generated frequencies of traits are already an index of everyday accessibility in the particular sample in which it has been produced. Hence, a prototype defined in terms of accessibility and operationalized through the frequency of output, can also be delivered by a 'nominator' sample.

The content of a prototype has been referred to by various names such as features, characteristics, exemplars or attributes (Horowitz \& Turan, 2008). In this 
study, the associations with a 'moral person' can be understood as traits, for example, being honest or helpful (see also Smith, et al., 2007). However, the same examples can also be understood as values and have been included as such in a number of value surveys (e.g. Rokeach, 1973; Schwartz, 1992).

Traits refer to relatively stable characteristics of an individual that guide his/her behaviour. Traits can be merely descriptive of a person, but they can also relate more closely to values as 'ought' and 'should' in that a particular trait is good or bad, right or wrong, or that someone ought to have such a disposition (Kilmann, 1981). Values and traits have usually been researched separately (Bilsky \& Schwartz, 1994; Wojciszke, 1997). Roccas, Sagiv, Schwartz and Knafo (2002) pointed out that the same term may refer either to a trait or a value. They emphasize, however, that there is a difference in their psychological meaning. For instance, 'competence' may be a guiding principle in people's life (a value), but not all individuals share the trait of behaving 'competently', and vice versa. This distinction is important when investigating individual differences in traits and their link to personal values in the way Roccas and colleagues have done. However, for the present study the distinction of traits and values becomes trivial. What is studied here are the group-generated associations with a 'moral person' and not the differential characteristics of participants. Hence, the output may as well be considered as a set of values, and more specifically as moral values since the associations refer to attributes of a 'moral person'. In this sense, the present study can be regarded as an implicit measure of moral values. 
Research in Moral Psychology

Walker and colleagues (Walker \& Frimer, 2007; Walker \& Hennig, 2004; Walker \& Pitts, 1998b) were the pioneers in the analyses of prototypes in the area of morality. Walker and Pitts (1998) used the two-step approach of 'nominators' and 'raters' to generate attributes of a highly 'moral person'. In a subsequent study, they employed a similarity sorting task and applied multidimensional scaling techniques to uncover people's conception of a moral exemplar. They found that moral attributes were arranged along two dimensions: the first dimension characterized attributes that refer to the 'self' or 'others'; the second dimension showed attributes of 'internal' versus 'external' moral standards. Hence, individuals' knowledge of a moral person could be distinguished along these two dimensions. Since their study explored individuals' conceptions of a moral person in a Western cultural context (Canada), the authors called for additional research to uncover moral prototypes in different cultures.

\section{Research in Cross-cultural Psychology}

Smith et al. (2007) followed Walker and Pitt's (1998) call for cross-cultural research on moral prototypes. They employed a feature-listing procedure in seven different cultures (Chamorro, Filipino, Taiwanese, Turkish, US-American, Venezuelan, and Palauan). Their choice of countries consisted of individualistic (e.g. AngloAmericans) and collectivistic-oriented samples (e.g. Filipinos) as well as samples from urban and rural areas and those belonging to multiple religious traditions (e.g. Catholics and Muslims). Instead of using the 'moral person' as a target to elicit free-listings (Walker \& Pitts, 1998), they used the 'good person' as a target. Their results showed that attributes of the 'good' person overlapped to some extent across cultures. However, a cross-cultural comparison of the rank orders of the trait frequencies showed considerable variation. In an attempt to interpret the meaning of the 'good person' 
prototype, they matched the free-listed attributes with experts' traits and values classifications and conducted a content analysis. Their results showed that (1) interpersonal benevolence (caring, humanity) dominated as the most accessible attribute of a 'good person' across all cultural samples, (2) qualities of the competence-kind category (e.g. achievement, confidence, drive) varied widely across cultures in terms of accessibility. They also correlated the trait frequencies with Walker and Pitt's (1998) two-dimensional coordinates on attributes of a moral person. They found that the 'other'- and 'internal'-relevant pole significantly correlated with the frequencies of freelisted attributes of their 'good person' target. Hence, interpersonal qualities, as well as those not referring to others, seem to be related to the trait accessibility of a 'good' person.

Despite their great effort and approach in studying moral prototypes across cultures, there still remain questions, some of which I will address in the present study.

\section{The Present Study}

The present study aims to investigate moral prototypes across cultures and to address some of the limitations of Smith, et al.'s (2007) research. Smith and colleagues used the 'good person' as a target to elicit a moral prototype. However, one may argue that a moral prototype should relate to the specific concept of a 'moral person'. Hence, the present study aimed to answer the following questions:

RQ1: What kinds of attributes are associated with a 'moral person'?

RQ2: Are there cultural differences and similarities in associations of a 'moral person'?

In regard to $R Q 2$, it was expected that cultural groups that can be classified as individualistic or collectivistic based on prior research (e.g. Hofstede, 1980) would show qualitatively different moral prototypes. If morality is closely related to culture - 
which is an underlying assumption in this thesis (see chapter one for more details) - then there should be a difference between moral prototypes produced by individualistic and by collectivistic samples. However, what kind of differences are to be expected? Shweder and colleagues' (1997) theory on moral discourse across cultures may provide an indication. They proposed, and showed empirically, that moral discourses can be distinguished cross-culturally into the ethics of Autonomy, Community, and Divinity ${ }^{23}$. The former is more strongly endorsed in individualistic countries, and is based on moral reasoning in terms of individual rights and subjective well-being. The latter two, on the other hand, are more endorsed in collectivistic countries and represent a moral reasoning with reference to duties/ social obligations and spirituality/ purity respectively. Hence, it was expected that the most accessible moral attributes produced by individualistic and collectivistic cultural groups would differ in regard to these three categories.

Since the prototype analysis of a 'moral person' is regarded as an implicit measure of moral values, there is the unique opportunity to study its relationship with an explicit measure of moral values from study three of this thesis (obtained through selfreported ratings on the moral relevance of values). Hence, the research question was:

RQ3: Are implicit and explicit measures of moral values related to each other?

It was expected that the group-generated frequencies of the implicitly assessed moral values would correlate positively with the sample-based societal moral value hierarchy, as assessed in Study 3. If implicit moral values reflect a form of 'collective wisdom' about right and wrong which is presumably subject to cultural variation, then they should also be related to societal expectations in the respective cultural sample. Personal values should not be correlated with the frequencies of moral attributes since they are conceptually unrelated to morality (see introduction of this thesis for more

\footnotetext{
${ }^{23}$ See chapter one and three for more details on Shweder et al's (1997) theory.
} 
details). Initially, there were no specific expectations in regard to personal moral values. However, since the personal moral value hierarchy proved to be very similar across cultures in Study 3, it was not expected that these values would be related to implicit moral values which are supposed to assess a form of 'collective' or 'cultural wisdom'.

The last research question of this study concerned the question whether the 'good person' and the 'moral person' are interchangeable targets to elicit moral attributes.

RQ4: Does the 'good person' elicit the same characteristics as the 'moral person'?

Smith, et al. (2007) used the 'good person' as a target to elicit moral prototypes across cultures. Their rationale for this target choice was that 'good' is a fundamental concept in all cultures and that attributes that are central to meeting the challenges of life within a particular culture are likely to be the ones that discriminate between good and less good persons. However, a legitimate and important question is whether targets are interchangeable. Smith, et al. (2007), for example, found only partial overlap between their list of attributes and Walker and Pitts' moral attributes. The discrepancy between the 'good person' and 'the moral' person as targets was even more accentuated in a study conducted by Lapsley and Lasky (2001). The authors compared their freelistings of the 'good character' to Walker and Pitts' (1998) moral attributes and found that the two lists shared only the feature of honest. They concluded that it may not be justified to equate the target of a 'moral person' with the target of a 'good person'. It is possible that the 'good person' is a more general concept whereas the 'moral person' elicits more specific concepts related to issues of morality. The good-bad distinction as a cue may, for example, elicit not only what is socially good-bad (morality-related), but also what is intellectually good-bad (competence-related) (see Rosenberg \& Sedlak, 
1972). A limitation of Smith, et al.'s (2007) cross-target comparison with Walker and Pitts' findings is that the two datasets are based on different cultural groups. Hence, the mere partial overlap between the two datasets cannot only be explained with the use of different targets, but also with the use of different cultural samples. In order to rule out culture as a competing explanation for identified difference in target use, free-listings of the 'good' and the 'moral' target were compared between samples from the same cultural background.

To summarize, the goals of this research were:

(1) to describe and compare cross-culturally laypersons' concepts of the moral person in terms of the features that are accessible to them;

(2) to compare the group-generated traits of a 'moral person' to explicit measures assessing personal and moral values.

(3) to compare features of the 'good' and the 'moral' target.

This study is the first to investigate implicit knowledge about moral attributes cross-culturally.

\section{Method}

\section{Participants}

Ethics approval for this study was granted by the Victoria University of Wellington Human Ethics Committee. Participants were recruited from four different countries. The choice of countries was determined by sampling individuals from collectivistic countries (Brazil and the Philippines) and individualistic countries (Germany and New Zealand; Hofstede, 1980; Schwartz, 2006b) belonging to different parts of the world. As already pointed out in the previous chapter, geographical distance may be a rival explanation for cultural differences (cf. van de Vijver \& Leung, 1997). However, if differences identified between individualistic and collectivistic countries 
generalize across samples from different parts of the world, it can be ruled out that they are solely due to geographical distance. Instead the conclusion can be drawn with more confidence that the differences are attributable to the underlying cultural dimension individualism-collectivism.

A total of 540 university students from the social sciences participated in this study. Respondents were only included in the analysis if they identified with the country of residence as assessed in the survey. Hence, responses were analyzed from 98 Brazilians, 119 Germans, 108 Filipinos and 172 students from New Zealand (see Table 6.1 for more details about the samples). There were significant age differences across the five samples, $F(4,497)=56.18, p<.001$, and also differences in the proportion of females, $\chi^{2}(4)=24.01, p<.001$. Respondents' average age was highest in Brazil and Germany $\left(M_{\text {Brazil }}=23.62, S D=4.77 ; M_{\text {Germany }}=23.61, S D=4.94\right)$ and lowest in the Philippines $(M=17.32, S D=1.15)$. These age groups correspond roughly to differences in student profiles as a result of different university entry requirements in each country. The New Zealand sample that received the moral target showed the highest percentage of females (71\%) and the Brazilian sample the lowest (49\%). The samples reflect the typical gender composition in the social sciences with females being often in the majority. It was surprising that one of the New Zealand sample was composed of almost three-quarter of females, whereas the other New Zealand sample which was recruited simultaneously exhibited a considerably lower proportion of females (59\%). Since the questionnaire was distributed randomly to these participants, the most likely explanation for the differences in gender composition is chance alone. 
Table 6.1: Sample characteristics of Study 4.

\begin{tabular}{lcccccc}
\hline & & Brazilian & German & Filipino & $\begin{array}{l}\text { New Zealand } \\
\text { (moral target) }\end{array}$ & $\begin{array}{l}\text { New Zealand } \\
\text { (good target) }\end{array}$ \\
\hline$N$ & & 98 & 125 & 119 & 90 & 82 \\
Age & Mean & 23.62 & 23.61 & 17.32 & 19.02 & 19.07 \\
Female & $(S D)$ & $(4.77)$ & $(4.94)$ & $(1.15)$ & $(3.33)$ & $(4.04)$ \\
\hline
\end{tabular}

Note. SD denotes 'standard deviation'.

\section{Materials}

Participants were first asked to provide demographic information. They were then given the moral person as the target concept and asked to write down what kind of characteristics they associate with it. New Zealand students received randomly either the 'moral' or the 'good person' as a target in their survey. The following instruction was given to the respondents and was adapted from Lapsley and Lasky (2001).

The following question has to do with the sort of things we have in mind when we hear and use words. For example, if you heard the word "fruit" you might think of such things as apples and pears. If you heard the word "furniture," you might think of sofa, couch, or table. If you heard the word "extrovert," you might think of outgoing, friendly, and sociable. What comes to mind when you think of a person who has "moral character"24?

The instruction was translated into German and Portuguese by the author, and its accuracy was verified with at least one bilingual colleague in both cases. The Filipino sample received the English version, since English is one of the official languages in the Philippines. Participants in New Zealand were asked to write down as many attributes they could think of within 10 minutes and were provided with a maximum of 20 lines for their open-ended answers (cf. Walker \& Pitts, 1998). This response format yielded in some cases very long and essay-like statements which rendered the answers more difficult to analyze. Hence, in the remaining three samples the response format was structured by providing six bullet points which could be completed with moral features.

\footnotetext{
${ }^{24}$ In the New Zealand sample about half of the students received the same instruction in which 'moral character' was replaced with 'good character'.
} 
The number of chosen bullet points corresponded to the average number of traits reported in the New Zealand sample in which a 'moral person' has been used as a target.

\section{Procedure}

Participants in New Zealand completed the feature-listing task within a mass testing session. Students in Brazil and in the Philippines responded to the survey in a class setting. For the data collection in Germany, the feature-listing was part of a survey not related to morality or moral attributes. For all samples it was ensured by the collaborators that participants listed the features silently and independently.

\section{Analytical Strategy}

An example of all original responses from one sample can be seen in Appendix F. Traits listed by German and Brazilian participants were translated into English by the author and bilingual consultants. Judgments rules (see also Fehr, 1988; Walker \& Pitts, 1998b) were developed in order to facilitate the final cross-cultural frequency analysis of moral attributes. Judgments rules were used in two different stages: the first stage consisted of an intra-cultural analysis; and the second stage of a pan-cultural analysis of free-listings. In the intra-cultural stage, frequencies of moral attributes were analyzed separately in each culture. The pan-cultural analysis consisted of pooling all free-listed features regardless of the respective cultural group in which they have been listed to identify universal categories that may underlie the data.

Figure 6.1 depicts the process for both stages. The aim during the whole procedure was to (1) reduce the list of descriptors as much as possible, while at the same time remaining as conservative as necessary to preserve culture-specific features, 
and (2) to fit the specific traits into more general attributes, so that a computerized frequency analysis could detect and count these attributes.

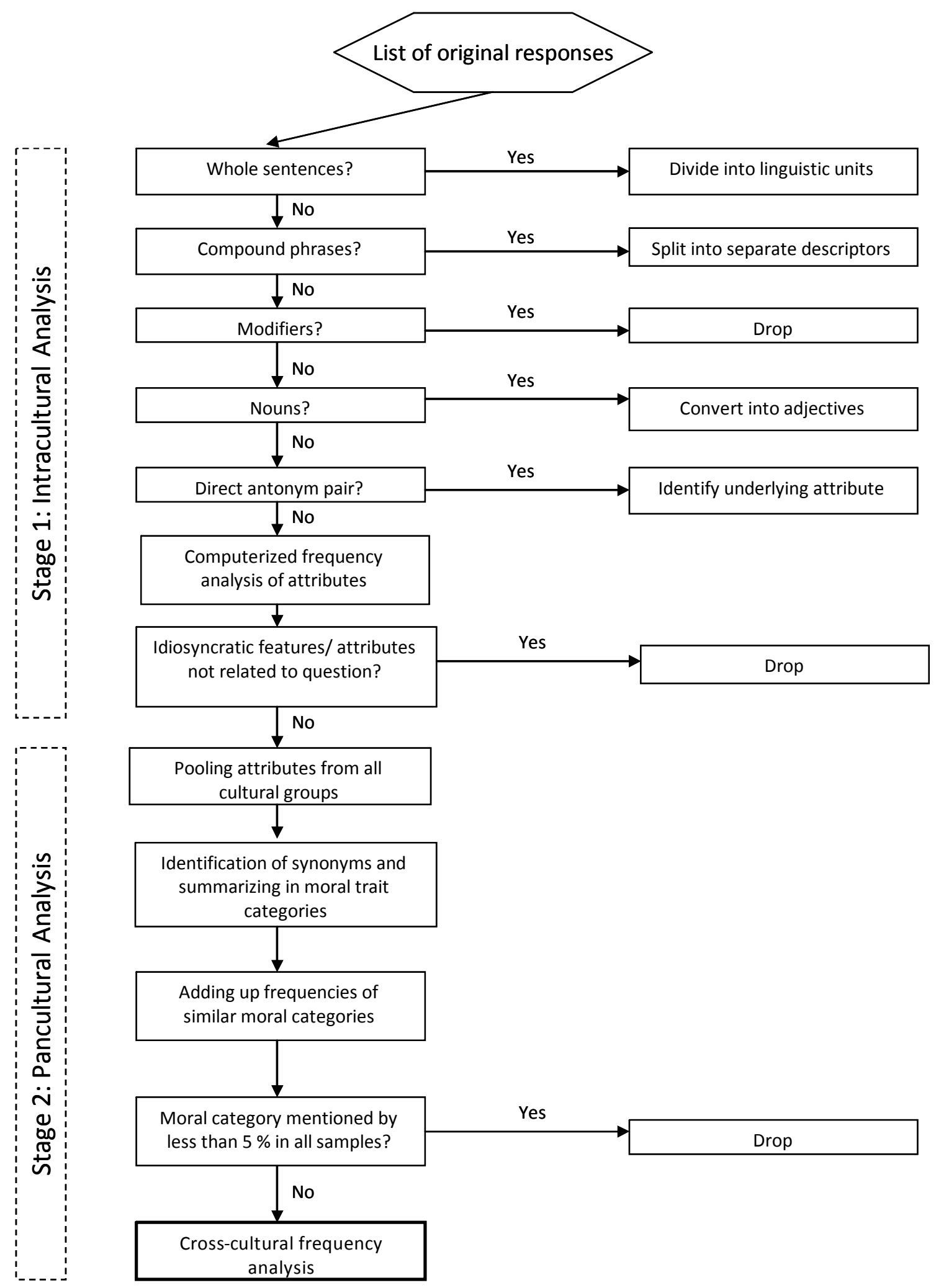

Figure 6.1: Judgment rules for the content analysis of moral attributes. 
The intra-cultural analysis consisted of the following steps: Dividing whole freelisted sentences into linguistic units; separating compound phrases into separate descriptors if they could stand alone (e.g. just and open-minded became just and openminded); dropping modifiers (e.g. very loving became loving); converting nouns into adjectives whenever possible (e.g. courage became courageous); and summarizing direct antonym pairs (e.g. fair and unfair were both counted under the attribute of fair since they belong to the same underlying meaning of fairness; decision about the polarity of this attribute was determined by the most frequently mentioned pole). The remaining attributes were then analyzed with the software program MAXQDA 2007 (Kuckartz, 2007) in terms of how frequently they occurred. Idiosyncratic features (i.e. those that were mentioned by only one person) and also attributes not directly related to the target were dropped. This was done separately for each sample.

The responses were then pooled across samples and further analyzed in a pancultural fashion. The main aim herein was to identify synonyms of moral attributes. These synonyms were then summarized under a moral trait category. Pooling the attributes across samples ensured that this could be done without 'cultural bias', i.e. without treating an attribute as culture-specific when in fact it was synonymous to a similar attribute mentioned in other cultural samples. For instance, stable was only mentioned in the Brazilian sample; however, well-balanced was identified as a synonym occurring in the German and Filipino sample. Hence, the moral trait category was labeled well-balanced (because well-balanced occurred more frequently than stable) and Brazilian responses which yielded the trait stable were counted as falling under the category of well-balanced. The categorization of all moral traits was discussed with another colleague until agreement was achieved. The frequencies of the final moral trait categories were eventually added up. Only categories that were mentioned by at least 
five percent of the respondents in any one of the samples were retained and further analyzed cross-culturally.

\section{Results and Discussion}

\section{Comparing Moral Attributes across Cultural Groups}

The number of elicited traits were 512 in the Brazilian sample $(M=5.68, S D=$ $0.84), 608$ in the German sample $(M=5.79, S D=0.64)$, and 590 in the Filipino sample $(M=5.94, S D=0.31)$. The differences in the number of free-listed traits was significant $(F(2,335)=5.25, p<.01)$. In the New Zealand sample in which the unstructured response format encouraged participants to write as much as they wanted, there were 581 single attributes $(M=6.50, S D=3.97)$ and 157 sentences for the 'moral target' $(M$ $=2.71, S D=1.69)$ and 888 features for the 'good target' $(M=10.83, S D=5.01)$. Interestingly, the 'good' target survey yielded more single attributes than the 'moral' target and there were no whole sentences free-listed despite the same response format. It may be that the 'moral person' is a more complex concept than the 'good person' which stimulates individuals to provide more information on their associations and the underlying meaning. In contrast, the 'good person' as a stimulus seems to be more readily defined in terms of single uncontested meanings. For the sake of consistency in regard to the other cultural samples, only the first six traits were analyzed in the New Zealand sample, which yielded 496 attributes with the 'moral target' and 504 attributes with the 'good target'.

After applying the judgment rules described above, the descriptors were reduced to 49 distinct moral trait categories. Table 6.2 shows the features for all four cultural groups and the associated frequencies. Since there were significant differences in the number of free-listed traits, the frequencies were adjusted by the respective sample size and then expressed in percentages. 
Table 6.2: Attributes of a moral person in four cultures.

\begin{tabular}{|c|c|c|c|c|c|c|c|c|}
\hline & Brazilian & $\%$ & German & $\%$ & New Zealand & $\%$ & Filipino & $\%$ \\
\hline 1 & honest & 99 & honest & 53 & honest & 67 & religious & 66 \\
\hline 2 & just & 39 & just & 45 & good & 24 & good & 54 \\
\hline 3 & respectful & 33 & helpful & 24 & kind & 24 & honest & 40 \\
\hline 4 & educated & 22 & has standards & 23 & ethical & 22 & kind & 35 \\
\hline 5 & correct & 20 & open & 20 & trustworthy & 22 & respectful & 33 \\
\hline 6 & ethical & 20 & considerate & 19 & caring & 21 & right & 21 \\
\hline 7 & responsible & 18 & religious & 18 & has standards & 20 & well-mannered & 21 \\
\hline 8 & serious & 18 & critical & 17 & helpful & 19 & just & 19 \\
\hline 9 & good & 16 & obedient & 16 & friendly & 18 & friendly & 17 \\
\hline 10 & loyal & 15 & friendly & 13 & right & 18 & generous & 13 \\
\hline 11 & sociable & 15 & reliable & 13 & strong & 18 & obedient & 13 \\
\hline 12 & trustworthy & 14 & unselfish & 11 & considerate & 17 & ethical & 12 \\
\hline 13 & dignified & 12 & conservative & 10 & compassionate & 11 & has standards & 12 \\
\hline 14 & reasonable & 10 & tolerant & 9 & respectful & 11 & humble & 11 \\
\hline 15 & determined & 9 & well-mannered & 9 & proud & 10 & loving & 9 \\
\hline 16 & has standards & 9 & respectful & 8 & reliable & 10 & virtuous & 9 \\
\hline 17 & kind & 8 & sociable & 8 & firm & 9 & disciplined & 8 \\
\hline 18 & firm & 7 & conscientious & 8 & generous & 9 & exemplary & 8 \\
\hline 19 & humble & 7 & ethical & 8 & just & 8 & considerate & 7 \\
\hline 20 & personality* & 7 & kind & 8 & loyal & 8 & helpful & 7 \\
\hline 21 & conscientious & 6 & determined & 7 & obedient & 8 & trustworthy & 7 \\
\hline 22 & rigid & 6 & loyal & 7 & unselfish & 8 & caring & 6 \\
\hline 23 & disciplined & 5 & self-confident & 7 & respected & 7 & responsible & 6 \\
\hline 24 & obedient & 5 & firm & 6 & tolerant & 7 & determined & 6 \\
\hline 25 & self-confident & 5 & good & 6 & intelligent & 6 & unselfish & 6 \\
\hline 26 & helpful & 4 & intelligent & 6 & religious & 6 & wise & 6 \\
\hline 27 & loving & 4 & trustworthy & 6 & well-mannered & 6 & compassionate & 5 \\
\hline 28 & respected & 4 & correct & 5 & reasonable & 4 & dignified & 5 \\
\hline 29 & considerate & 3 & easy-going & 5 & responsible & 4 & loyal & 5 \\
\hline 30 & critical & 3 & reasonable & 5 & self-confident & 4 & conscientious & 4 \\
\hline 31 & friendly & 3 & responsible & 5 & virtuous & 4 & educated & 4 \\
\hline 32 & intelligent & 3 & exemplary & 4 & determined & 3 & self-confident & 4 \\
\hline 33 & religious & 3 & right & 4 & correct & 2 & sociable & 4 \\
\hline 34 & strong & 3 & rigid & 4 & dignified & 2 & respected & 3 \\
\hline 35 & open & 2 & disciplined & 3 & exemplary & 2 & intelligent & 2 \\
\hline 36 & virtuous & 2 & loving & 3 & rigid & 2 & open & 2 \\
\hline 37 & exemplary & 1 & strong & 3 & sociable & 2 & correct & 1 \\
\hline 38 & right & 1 & compassionate & 2 & wise & 2 & critical & 1 \\
\hline 39 & wise & 1 & respected & 2 & educated & 1 & easy-going & 1 \\
\hline 40 & caring & 0 & serious & 2 & humble & 1 & firm & 1 \\
\hline 41 & compassionate & 0 & caring & 1 & loving & 1 & reasonable & 1 \\
\hline 42 & conservative & 0 & generous & 1 & conscientious & 0 & reliable & 1 \\
\hline 43 & easy-going & 0 & humble & 1 & conservative & 0 & rigid & 1 \\
\hline 44 & generous & 0 & dignified & 0 & critical & 0 & strong & 1 \\
\hline 45 & proud & 0 & educated & 0 & disciplined & 0 & tolerant & 1 \\
\hline 46 & reliable & 0 & personality* & 0 & easy-going & 0 & conservative & 0 \\
\hline 47 & tolerant & 0 & proud & 0 & open & 0 & personality* & 0 \\
\hline 48 & unselfish & 0 & virtuous & 0 & personality* & 0 & proud & 0 \\
\hline 49 & well-mannered & 0 & wise & 0 & serious & 0 & serious & 0 \\
\hline
\end{tabular}




\section{Central and Peripheral Moral Attributes}

To obtain a quantitative measure of similarity for the 49 moral trait categories across cultural groups, the sample profiles were correlated with each other. Significance tests for product-moment correlation coefficients assume that the population is normally distributed. However, the frequency counts of moral attributes were skewed (combined sample: skewness $=3.7$, range $=1-65, M d n=6.1$ ). Therefore, Spearman correlation coefficients were computed. The results are summarized in Table 6.3. The coefficients show that all cultural profiles are different from each other, except for the Filipino and New Zealand sample. In line with Smith and colleagues' (2007) study on 'good' character traits across cultural groups, the correlations in this study were also consistently fairly low and tend to indicate cultural differences rather than similarities. The moral attribute profile of the two individualistic cultures New Zealand and Germany was marginally correlated with each other. The finding that the frequencies of the free-listed features from the New Zealand and Filipino sample were significantly similar to each other was surprising, even though the correlation was only of moderate size $\left(r_{s}=.40, p<.05\right)$. Positive correlations of frequencies were expected between individualistic or collectivistic samples, but not across them.

It may be that the individualism and collectivism distinction of samples is more pronounced when examining central moral features as opposed to all moral features. Central moral features were operationalized as those that are highly accessible and therefore occur with a relatively high frequency compared to non-central traits (cf. Gregg, et al., 2008; Smith, et al., 2007). Hence, central moral trait categories were examined next and compared cross-culturally. 
Table 6.3: Correlations between percentages of free-listed moral features across four cultures.

\begin{tabular}{lllll}
\hline Sample & 1 & 2 & 3 & 4 \\
\hline 1. Brazilian & - & & \\
2. German & .09 & - & - & \\
3. New Zealand & .11 & .29 & $.40^{*}$ & - \\
4. Filipino & .12 & .21 & .27 & .24 \\
Mean $r_{s}$ & .11 & .20 & ${ }^{\dagger}$ & .05, two-tailed. Correlations are Spearman coefficients. Features having zero \\
\hline Note. ${ }^{\dagger} p<.10,{ }^{*} p<$. & \\
frequency in either of the given two samples were excluded from the analysis. Hence, degrees of freedom \\
and critical values for coefficients vary depending on the number of features with nonzero frequencies (N \\
$=33-45)$. Correlations based on all features including $(0,0)$ data pairs $(\mathrm{N}=49)$ were very similar to the \\
correlations in the table above.
\end{tabular}

Central Moral Attributes

Past research has usually identified central features by asking an independent sample to rate the features that the so-called 'nominator' sample ${ }^{25}$ has produced (Horowitz \& Turan, 2008). However, even within this approach there is little agreement about how many attributes can be considered as making up central features of a prototype (cf. Fehr, 1988; Lapsley \& Lasky, 2001). Furthermore, there is no established approach in the literature that determines centrality of features purely based on frequency data.

Researchers using the Natural Semantic Network technique employ the scree test to determine the network core in order to investigate the psychological meaning of a given concept (Milfont, personal communication, July 16, 2008). Although semantic networks and free associations are products of different processes (Figueroa, González, \& Solís, 1976), a scree test may be useful for the latter in order to differentiate between central (frequent) and peripheral (non-frequent) trait categories.

A scree test (Cattell, 1966) is usually employed in factor analysis in which eigenvalues are plotted against factors. Factors are arranged in descending order along the abscissa and eigenvalues on the ordinate. This forms a descending curve. The

${ }^{25}$ The 'nominator' sample is the sample that free-lists characteristics of a specific target. 
number of factors to interpret is the point where this curve shows an 'elbow' (change in gradient). A similar approach can be used to determine the centrality of free-listed features. Here, the relative frequencies can be arranged in descending order along the ordinate and the features along the abscissa.

An example of a scree plot for two cultural groups is depicted in Figure 6.2. The figure shows a somewhat different pattern for the sample from New Zealand compared to the sample from the Philippines. In New Zealand, the first moral category yielded a very high relative frequency compared to subsequent categories. Hence, the 'elbow' would indicate that only one moral feature is central. For the Filipino sample on the other hand, the 'elbow' appears after about six moral trait categories. The figure also shows clearly that when moving to the end of the ordinate, there are practically no bigger drops in relative frequencies. Hence, it appears that the 'conventional' use of the scree plot would suppress a great deal of additional information, especially in the case of the New Zealand sample.

Another point to consider is that information processing theory suggests that we can process 5-9 chunks of information (Miller, 1956). Hence, it is likely that the number of moral features that are distinct from each other, but central to people's perception of a moral person is close to seven plus or minus two; and in fact, the average number of traits mentioned in the New Zealand sample, i.e. under the non-structured condition, was around six traits. On the other hand, central features should also occur with considerable higher frequencies than peripheral features. Therefore, the following judgment rule were developed to identify centrality of free-listed moral categories: (1) the category needed to be mentioned by at least ten percent of the sample, (2) after this ten percent threshold and following the increasing frequencies along the ordinate, the next largest increase in frequencies was identified with the aid of the scree plot, (3) the number of central traits should be not much less than five and not substantively more 
than nine. This procedure yielded eleven central categories in the Brazilian and German sample, twelve in the New Zealand and nine in the Filipino sample (see Table 6.2.).

The results show that honest was a 'universal' central moral trait category (occurring in all four countries), followed by friendly, good and just (central in three countries). Culturally idiosyncratic central moral categories were open, critical, reliable and obedient in the German sample and correct, serious, educated, responsible, loyal and sociable in the Brazilian sample. Well-mannered was an idiosyncratic central moral category in the Filipino sample and caring, strong, and trustworthy were central and specific to the New Zealand sample.

Spearman correlations of central moral attributes yielded a similar picture to the correlations of all 49 moral trait categories (see Table 6.4). Correlations did not reach significance in any of the samples, except for the New Zealand and Filipino sample which showed a marginally significant correlation of .41 $(p=.05)$. Hence, in regard to the research question whether there are cultural differences in associations of the 'moral person', it seems that there are indeed pronounced cultural differences.

Table 6.4: Correlations between percentages of free-listed 'central' moral features across four cultures.

\begin{tabular}{|c|c|c|c|c|}
\hline Sample & 1 & 2 & 3 & 4 \\
\hline 1. Brazilian & - & & & \\
\hline 2. German & -.03 & - & & \\
\hline 3. New Zealand & -.12 & .01 & - & \\
\hline 4. Filipino & .14 & .21 & $.41^{\dagger}$ & - \\
\hline Mean $r_{s}$ & .00 & .06 & .10 & .26 \\
\hline \multicolumn{5}{|c|}{$\begin{array}{l}\text { Note. }{ }^{1} p<.10 \text {, two-tailed. Moral attributes may be central in one culture, but peripheral in another. In any } \\
\text { case, all frequencies associated with the traits in question were taken into account. Frequencies of features } \\
\text { having zero frequency in either of the given two samples were excluded from the analysis. Hence, } \\
\text { degrees of freedom and critical values for coefficients vary depending on the number of features with } \\
\text { nonzero frequencies }(\mathrm{N}=20-25) \text {. Correlations based on all features including }(0,0) \text { data pairs }(\mathrm{N}=26) \\
\text { were very similar to the correlations in the table above. }\end{array}$} \\
\hline
\end{tabular}



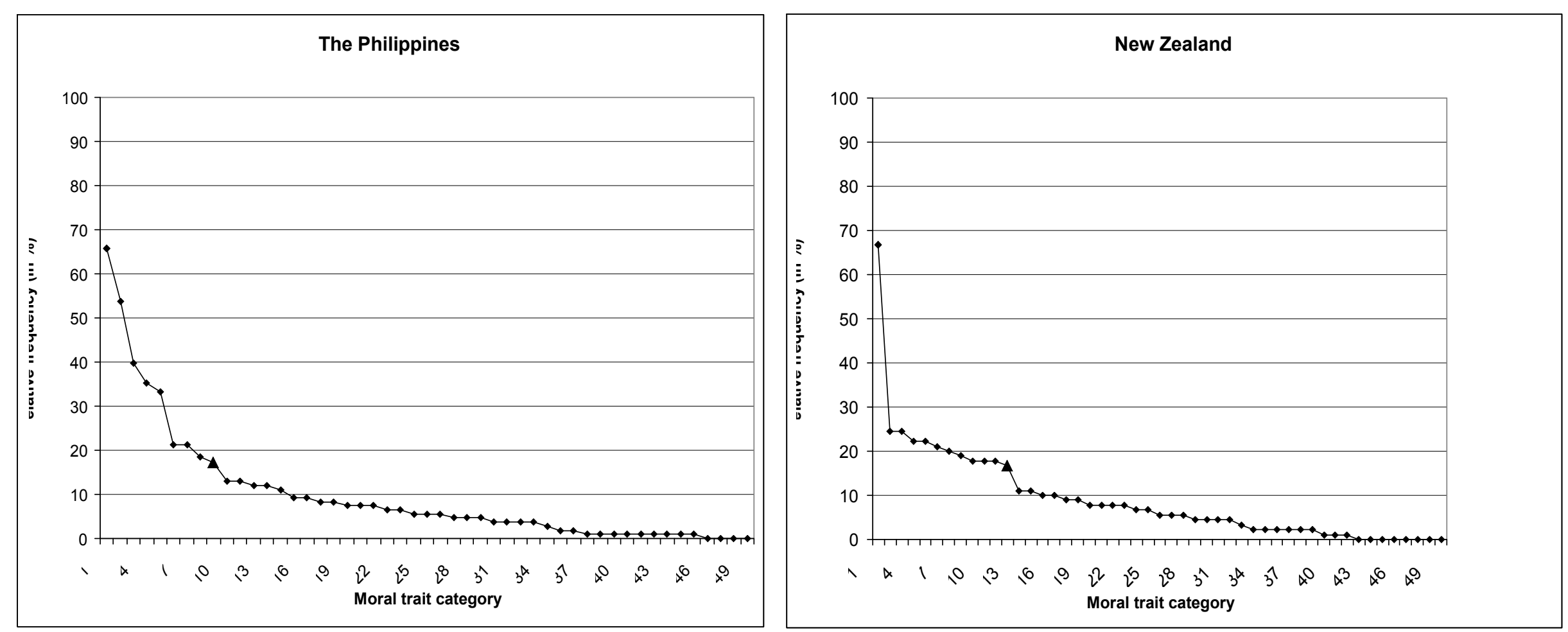

Figure 6.2: Examples of frequency scree plots of free-listed moral attributes in two samples

Note. The triangle symbol indicates the limit between moral trait categories determined to be central or peripheral. Relative frequencies are expressed in percentages. They do not sum to $100 \%$ as respondents were encouraged to list more than one attribute. 
In order to understand what kind of attributes are associated with a 'moral person' which may facilitate the interpretation of cultural differences, a correspondence analysis on central moral features was conducted.

\section{Interpreting Central Moral Attributes Cross-culturally}

\section{The Use of Correspondence Analysis}

Correspondence analysis is a multidimensional scaling technique that creates a perceptual map. Variables and objects are simultaneously plotted in the map based on the association between them (for technical details on this technique, see Greenacre, 1984; Hair, et al., 2006). Similarly to traditional multidimensional scaling techniques, it has only a few assumptions. Since it is a compositional technique, i.e. it combines separate attribute evaluations into an overall evaluation, the attributes should be as complete as possible (Hair, et al, 2006). This means that the analyzed attributes should be a good approximation of all attributes which would fully describe the construct. This assumption should be fulfilled when data is based on open-ended responses as it is the case in this study.

The underlying principle is similar to factor analysis in the sense that it allows factoring variables and determining the amount of explained variance for individual dimensions and the solution as a whole. However, in contrast to factor analysis, nonmetric data such as cross-tabulated frequencies can be used. Correspondence analysis is particularly useful when the two-way contingency table contains many rows and columns which may render it difficult to detect dependencies. These dependencies can be more easily detected by examining the perceptual map which displays patterns of association between objects and variables in a joint space: objects (here cultural samples) that are highly associated with certain variables (here moral trait categories) 
are located closer to them and farther away from variables with which they are not or not as much associated.

The perceptual map is produced by using chi-square distances between row and column profiles. The maximum possible number of dimensions is one less than the smaller of the number of rows or columns. A perfect representation can be obtained with the maximum number of dimensions possible. However, there is a trade-off between incremental explanation gained and interpretability of the solution. It is much easier to interpret the plot in a low- or preferably two-dimensional space. Whether a low-dimensional solution is appropriate can be examined by the proportion of total 'inertia' explained by the dimensions. In SPSS, the total 'inertia' is the variance and reflects the spread of points around the centroid, i.e. the origin of the correspondence map. Additional dimensions that do not add substantially to the explanation of the total inertia may be dropped. A rule of thumb is that dimensions with inertia lower than .20 may be excluded from the analysis (Hair, et al., 2006, p. 671). The dimensions are best interpreted by the points which contribute the most to the inertia of the respective dimensions. This is similar to interpreting factor loadings in conventional factor analysis. It is also worth pointing out that points that are close to the centroid have the least inertia. This means that they do not play a great role in defining the dimension. Applied to this study it means, that moral trait categories located close to the centroid represent attributes which occur with similar frequencies across the four samples.

A biplot is commonly used as a visual representation of the data. It displays both row and column points in a single multidimensional map. In order to directly compare frequencies of column and row profiles they need to be standardized which is called symmetrical normalization. Yet, it is important to note that even under this standardization procedure, one cannot precisely interpret the distance between a row point and a column point. It is preferable to make rather general interpretations, for 
instance, whether row and column points (or objects and variables respectively) appear in the same quadrant of the multidimensional space.

\section{The Resulting Biplot}

A correspondence analysis in SPSS 14 on the central moral trait categories (variables) across four cultural samples (objects) was conducted. In some cases, moral trait categories were central in some cultural groups, but peripheral in others. In these cases, the associated frequencies of culture-specific peripheral trait categories were also included in the analysis. The correspondence analysis produced a significant chi-square $\left(\chi^{2}(75)=745.23, p<.001\right)$ indicating that the total inertia was significantly different from zero. This means that there was a significant relationship between the samples and moral trait categories. Hence, it was justified to interpret the perceptual map in order to identify the nature of these dependencies. The total inertia was high indicating that the three dimensions explained $50.7 \%$ of the variance in the original correspondence table. The first dimension explained $45 \%$ of the $50.7 \%$, the second $30.9 \%$ of it and the third dimension 24\%. Even though the third dimension added a considerable amount of explained variance, the first two dimensions already allowed explaining about threequarters of the total inertia. Hence, to facilitate the interpretation of the perceptual map, it was decided to retain only two dimensions. The resulting two-dimensional biplot is depicted in Figure 6.3.

A striking feature of the map is its triangular shape. The samples from Germany, the Philippines and Brazil form the endpoints of this triangle. Points further from the origin play a greater role in defining the dimension, whereas points located close to the origin represent shared variance. The latter seems to be the case for the New Zealand sample which is located closely to the centroid. The position of the New Zealand sample may be interpreted as sharing common variance with all other samples. This is a 
somewhat different interpretation than the previous one based on Spearman correlations between moral trait frequency profiles in which the conclusion was drawn that the New Zealand sample is only similar to the Filipino sample. The similarity between these two samples is also replicated in the biplot in which they are relatively close to each other. However, it seems that the Spearman correlations which only consider the rank-ordering of moral trait categories did not detect the various dependencies that exist between the New Zealand sample and all other samples.

All samples exhibited some culture-specific central moral trait categories. However, the triangular shape of the biplot appears to be due to the responses of the German, Filipino and Brazilian sample. It may be explained by the fact that culturally idiosyncratic traits from these three samples occurred with very high frequencies in the respective samples and very low frequencies in all the other samples; this tendency was somewhat less accentuated in the case of New Zealand. Note that trustworthy, for example, emerged as an idiosyncratic central trait in New Zealand and occurred with a lower frequency in all other samples, however not as low as 0,1 or 2 as, for instance, the Brazilian-specific trait serious (cf. Table 6.2).

Taking the attributes in the triangle of the biplot as an anchor for interpretation of dimensions, dimension one may be interpreted as a 'self' versus 'other' dimension. Dimension two can be regarded as a 'liberal' versus 'traditional' orientation. There is a general tendency that the traits cluster on the 'other'-oriented side of the map which is associated with the German, New Zealand and Filipino sample. The 'self'-oriented elements were more specific to the Brazilian sample. Moral attributes also tended to cluster around the 'traditional' pole of the map which shows associations with the Filipino and Brazilian sample. Their perception of a moral person seem to reflect a more conservative kind of morality targeted at maintaining already existing moral standards. The German sample defined the opposite pole of dimension two labelled as 'liberal'. It 
is characterized by items such as being open and critical which may indicate a more autonomous and thoughtful handling of moral standards.

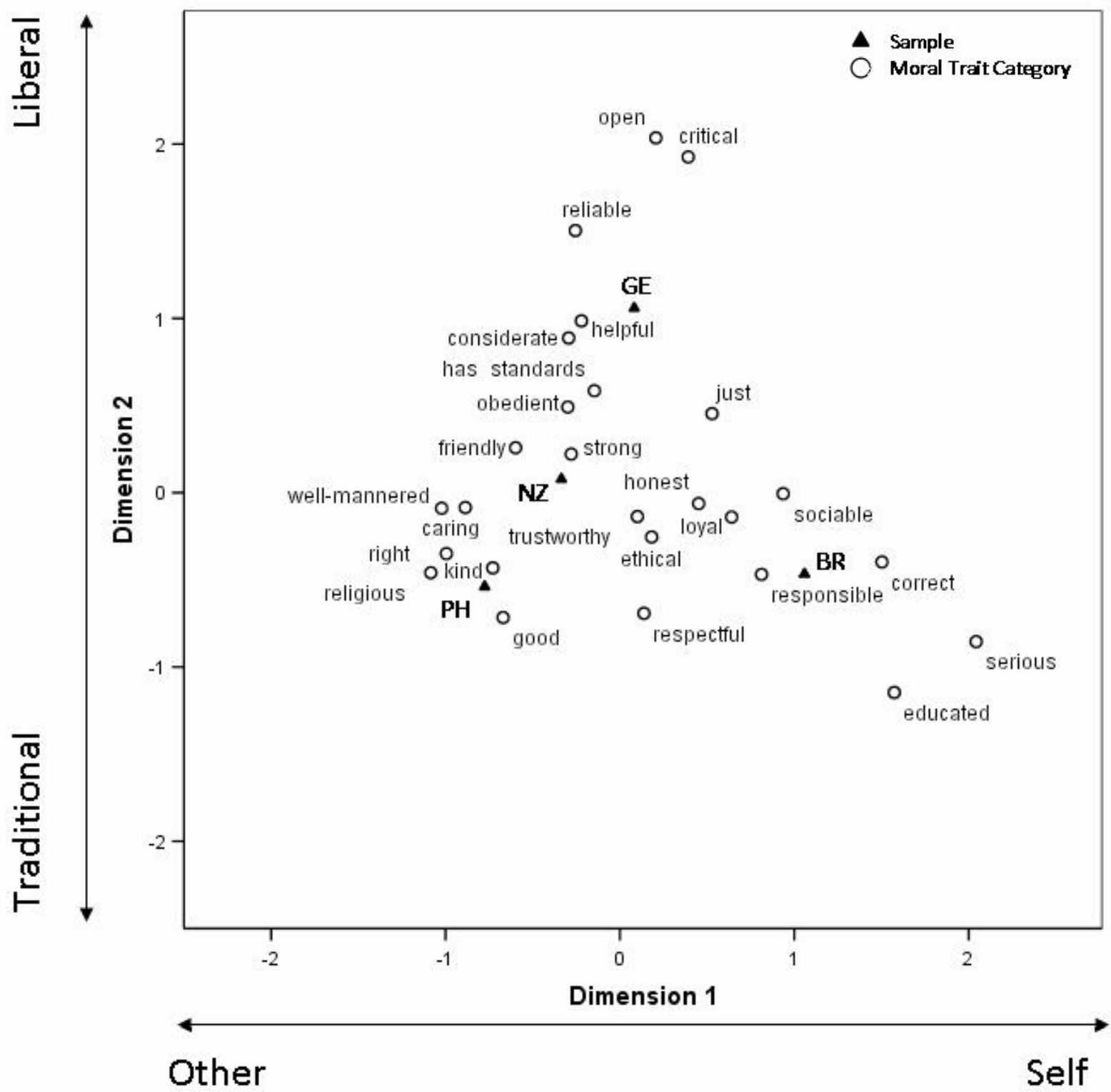

Figure 6.3: Biplot of relative frequencies of moral trait categories across four cultural samples.

Note. BR, GE, NZ and PH denote samples from Brazil, Germany, New Zealand and the Philippines respectively. 
A conclusion that can be drawn is that moral attributes tend to belong to the traditional kind of traits focused on the welfare of 'others'. Cultural differences and similarities on the two dimensions may be interpreted in line with Shweder et al.'s (1997) distinction of an ethic of Autonomy and Community/ Divinity. Traditional kinds of attributes that are oriented towards the individual fitting into the social group, i.e. an ethic of Community, can be regarded as widely shared across all samples (attributes are located close to the centroid in the biplot). The German sample, representing an individualistic culture, shows a culture-specific tendency that may indicate a form of ethic of Autonomy. However, autonomy is here not so much about the acknowledgment of individual rights as in Shweder and colleagues (1997) theory and findings, but about the person's ability to critically reflect about what is right and wrong in a selfdetermined way (e.g. critical, open). It also appears from the biplot that an ethic of Community goes hand-in-hand with an ethic of Divinity (represented by the moral trait category religious).

There was also an interesting culture-specific orientation that cannot be explained within Shweder, et al.'s (1997) typology. The Brazilian sample associated 'self-related' concepts with a moral person, such as being serious. Despite these traits being related to the 'self' they share little similarity with Shweder's ethic of Autonomy. They are better understood as personality traits related to the very 'self' of moral exemplars which differentiate them from others. Even though Walker and Pitts (1998) reported also a self-other dimension in their data, the content of their dimension differs from the present study. Walker and Pitts' (1998) self-focused attributes are more directly related to moral values (e.g. being righteous, having high standards, p. 414). The self-focused associations of the Brazilian sample (e.g. being educated, serious) are distantly related to the concept of a 'moral person' and could in fact be applied to a number of other persons, too. 
The findings from the correspondence analysis provide an intriguing insight into the associations between cultural samples and the underlying dimensions on which they are similar and different respectively. However, before a finding can be regarded as established evidence, it needs to be replicated (Amir \& Sharon, 1990). The aim of the following analyses is to validate the triangular shape of the biplot. This was done by examining whether the result is dependent on the attributes to be included in the analysis (cf. Hair, et al., 2006).

\section{Replicating the Biplot}

There are two possible reasons why the perceptual map displays a triangular shape. First, correspondence analysis is sensitive to outliers. The prevalence of culturespecific moral trait categories in the Brazilian, German and Filipino sample may have distorted the graphical association of objects. Even if 'outliers' provide valuable information regarding the prevalence of cultural differences and idiosyncrasies they may also lead to an overestimation of cultural dissimilarities in the perceptual map. Hence, consideration of a correspondence analysis without 'outliers' is highly worthwhile. Secondly, one of the few assumptions of correspondence analysis is that attributes should be complete, i.e. a good approximation of the items that would fully describe the construct. Adding or deleting categories may change the results (Hair, et al., 2006). In the analysis above, only central moral traits had been examined. An analysis that includes all 49 moral trait categories may produce another picture of cultural similarities and differences. Hence, these two analyses were also conducted in an attempt to validate the results.

The first analysis excluded all 14 culturally idiosyncratic moral trait categories. The chi-square was still significant $\left(\chi^{2}(33)=336.02, p<.001\right)$, but the inertia (variance) of .32 was somewhat less than in the previous analysis. Dimension 1 accounted for 
$54.7 \%$ of the inertia, dimension 2 for $27.5 \%$ and dimension 3 for $17.9 \%$ which is consistent with the first correspondence analysis. The perceptual map can be seen in Appendix G. There was still a triangular shape indicating that the German and New Zealand sample are similar to each other, whereas the Brazilian and Filipino sample are located opposite to each other. Even though with this reduced number of items it is more difficult to interpret the dimensions, there is still a 'traditional-liberal' dimension and arguably also a less well-defined 'self-other' dimension. The location of the cultural samples in the map was replicated.

The second analysis was conducted using all 49 moral categories. The chisquare was significant $\left(\chi^{2}(144)=1018.32, p<.001\right)$ and the inertia was .55. Dimension 1 accounted for $41.9 \%$ of the inertia, dimension 2 for $33 \%$ and dimension 3 for $25.1 \%$ suggesting that a three-dimensional solution may be more adequate for the data. Note that including all moral categories only explained an additional $4 \%$ of variance compared to the correspondence analysis based on central moral categories. This supports the importance of the central moral trait categories.

For ease of interpretation and comparison, the perceptual map was created in a two-dimensional space and can be seen in Appendix G. The same triangular shape emerged as in previous analyses. The cultural groups were arranged in the same fashion as in the correspondence analysis on central trait categories. The interpretation of the underlying dimension is here facilitated through the higher number of items. In single cases items seem to be misplaced such as conservative which is associated with the German sample and located on the liberal pole of the underlying dimension. Yet, the general picture is relatively clear and confirms the very first interpretation. For instance, the 'self'-orientation pole which is associated with the Brazilian sample includes here compatible attributes such as having a personality (to be understood in the sense of 'backbone' or fortitude in English) and being dignified. 
To conclude, the relative association of the four cultural samples and the underlying dimensions on which they seem to differ or to be similar respectively can be regarded as replicated with different sets of moral trait categories (i.e. central traits without culture-specific attributes as well as all trait categories).

\section{Comparing Implicit Moral Attributes with Explicit Measures of Values}

In the following analysis, the findings of the implicit method for deriving moral trait categories were compared to an expert-designed explicit method of measuring values. The Schwartz Value Survey (SVS, Schwartz, 1992) was taken for this purpose and moral trait categories were matched to the values from the SVS. Appendix H shows

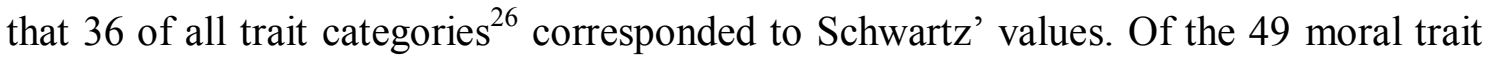
categories, there were 18 that could not be matched with specific Schwartz' values, which were: critical, easy-going, educated, ethical, exemplary, firm, friendly, generous, good, has standards, kind, has personality, reasonable, right, rigid, self-confident, serious, and sociable. When examining the matches between moral trait categories and value types, it was found that central moral attributes were predominantly from the value type benevolence and conformity (see Appendix H). Peripheral moral features were mostly from the universalism, tradition, and achievement value types. Hedonism did not occur at all. Hence, traits associated with power, security and stimulation as well as hedonism were not very salient characteristics of a person with moral character. Table 6.5 provides an overview of reported frequencies of moral trait categories falling under Schwartz' value types.

\footnotetext{
${ }^{26}$ To enhance the number of matches between moral trait categories and the SVS, traits that occurred with a frequency of less than five percent in all samples and which were excluded from the correspondence analysis earlier were here included.
} 
Table 6.5: Adjusted frequencies of matches between moral trait categories and Schwartz' value types.

\begin{tabular}{|c|c|c|c|c|c|c|c|c|c|c|c|}
\hline & $\begin{array}{l}\text { Pan- } \\
\text { cultural }\end{array}$ & & Brazil & & Germany & & $\begin{array}{l}\text { New } \\
\text { Zealand }\end{array}$ & & $\begin{array}{l}\text { The } \\
\text { Philippines }\end{array}$ & & $\begin{array}{l}\text { Pan- } \\
\text { cultural } \\
\text { Value } \\
\text { Hierarchy } \\
\text { (Schwartz } \\
\text { \& Bardi, } \\
\text { 2001) }\end{array}$ \\
\hline Schwartz' Value Types & $\Sigma \Sigma f / n$ & $\Sigma \Sigma f / n k$ & $\Sigma f / n$ & $\Sigma f / n k$ & $\Sigma f / n$ & $\Sigma f / n k$ & $\Sigma f / n$ & $\Sigma f / n k$ & $\Sigma f / n$ & $\Sigma f / n k$ & Mean rank \\
\hline Benevolence & 405 & 82 & 136 & 28 & 102 & 21 & 108 & 22 & 59 & 11 & 1 \\
\hline Conformity & 181 & 44 & 43 & 10 & 36 & 9 & 25 & 6 & 77 & 19 & 6 \\
\hline Universalism & 180 & 22 & 45 & 5 & 83 & 11 & 21 & 3 & 31 & 3 & 3 \\
\hline Tradition & 129 & 24 & 11 & 2 & 31 & 6 & 8 & 1 & 79 & 15 & 9 \\
\hline $\mathrm{X}$ & 65 & 5 & 15 & $<1$ & 11 & 1 & 24 & 3 & 15 & 1 & - \\
\hline Achievement & 38 & 9 & 10 & 2 & 10 & 2 & 7 & 3 & 11 & 2 & 4 \\
\hline Self-direction & 17 & 3 & 1 & $<1$ & 7 & 1 & 4 & 1 & 5 & 1 & 2 \\
\hline Stimulation & 8 & 3 & 0 & 0 & 3 & 1 & 3 & 1 & 2 & 1 & 8 \\
\hline Security & 3 & $<1$ & 0 & 0 & 0 & 0 & 1 & $<1$ & 2 & $<1$ & 5 \\
\hline Power & 3 & 1 & 3 & 1 & 0 & 0 & 0 & 0 & 0 & 0 & 10 \\
\hline
\end{tabular}

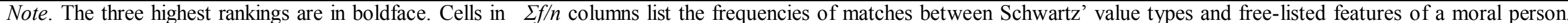

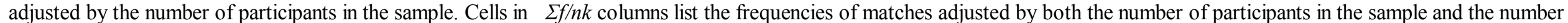

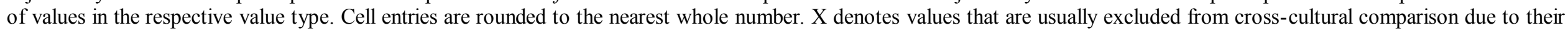
unstable meaning (Schwartz, 1992; 2007a). 
The mean Spearman correlation between cultural samples was relatively high with a value of .88 (for frequencies adjusted for the number of participants in the respective sample, $\Sigma \mathrm{f} / \mathrm{n}$ ) and .80 (for frequencies adjusted by both number of participants and number of values in the respective value type, $\Sigma \mathrm{f} / \mathrm{nk}$ ). Therefore, matching moral attributes with Schwartz' expert-designed value categories, shows that cultural groups are very similar in terms of the accessibility of moral values.

In order to examine whether accessibility of moral trait categories is indeed related to an expert-designed explicit theory, a procedure utilized by Smith, et al. (2007) was adopted. Multidimensional coordinates for Schwartz' personal value priorities were taken and correlated with the relative frequencies of moral attributes. For this purpose, Schwartz' data on value priorities from 26,024 students residing in 66 different countries were used. A two-dimensional solution was imposed on the pooled-within correlation matrix which weighs the correlation matrices of each country equally so that they have the same influence on the overall matrix (Fontaine \& Fischer, in press; Muthén, 1994) ${ }^{27}$. This pooled-within value structure has been found to be fairly stable (Fontaine, et al., 2008). Table 6.6 shows the results of the analysis. Dimension one of the SVS significantly correlated with the relative frequencies of moral trait categories, except for the New Zealand sample which could be due to the smaller sample size. This dimension is characterized by a personal vs. social value orientation (see also Fontaine, et al., 2008; Schwartz, 1992; Schwartz, 2005). Hence, moral trait categories were related to values with a social orientation. Dimension two of the SVS on the other hand did not correlate with moral trait frequencies in any of the samples. This dimension has been labelled as 'self-expansion and growth' versus 'self-protection against threat' value orientation (Schwartz, 2005). The results indicate that moral attributes that were matched with the SVS are not related to these kinds of self-focused values.

${ }^{27}$ See also chapter two for more details on sample characteristics of Schwartz' dataset as well as the multidimensional and pooled-within analyses. 
Table 6.6: SVS dimensions correlated with frequencies of moral trait categories.

\begin{tabular}{|c|c|c|c|c|c|}
\hline & \multicolumn{5}{|c|}{ Sample } \\
\hline & $\begin{array}{l}\text { Pan- } \\
\text { cultural }\end{array}$ & Brazilian & German & $\begin{array}{l}\text { New } \\
\text { Zealand }\end{array}$ & Filipino \\
\hline $\begin{array}{l}\text { SVS dim } 1 \\
\text { (personal vs. social) }\end{array}$ & $.36^{* * *}$ & $.36^{*}$ & $.46^{*}$ & .21 & $.45^{* *}$ \\
\hline $\begin{array}{l}\text { SVS } \operatorname{dim} 2 \\
\text { (growth vs. protection) }\end{array}$ & .05 & .23 & -.02 & .11 & .33 \\
\hline
\end{tabular}

A content analysis demonstrated that participants from all cultural samples accessed some of the value type categories more frequently than others (pan-cultural sample: $\chi^{2}(8)=266.09, p<.001$; Germany: $\chi^{2}(7)=53.54, p<.001$; New Zealand: $\chi^{2}(7)$ $=70.00, p<.001$; Brazil: $\chi^{2}(5)=66.75, p<.001$; The Philippines: $\chi^{2}(7)=56.13, p<$ $.001)^{28}$. Benevolence was assessed more frequently than other value types followed by conformity and universalism. One exception is the Filipino sample for which tradition and conformity value type categories were assessed most frequently.

In order to compare the implicit with the explicit measure of values, value type hierarchies based on frequencies (implicit measure) were correlated with value type hierarchies based on explicit ratings. There were five datasets of explicit value hierarchies with which the implicit measure could be compared to: (1) Schwartz and Bardi's (2001) pan-cultural value hierarchy based on students' importance ratings of values from 54 different nations (see also Table 6.5); and from study three of this thesis (2) personal importance ratings, (3) personal moral value ratings, and (4) societal moral value ratings derived from the cultural samples Brazil, Germany, New Zealand and the

\footnotetext{
${ }^{28}$ Chi-square statistics were computed on frequencies corrected for number of values in a value type.
} 
Philippines ${ }^{29}$. The correlations for the pan-cultural sample as well as for the four culture-specific samples are displayed in Table 6.7.

The Spearman correlations between the value type hierarchies produced by implicit and explicit measure of values showed that the implicit moral value measure was not related to personal importance ratings or perceived societal expectations. However, the implicit moral value measure was related to self-reported personal moral values. This correlation pattern was prevalent in both the pan-cultural sample as well as the four culture-specific samples. Two conclusions can be drawn: First, there is a relationship between implicit and explicit measures of moral values. This can be regarded as a validation of the measurement of moral values in the sense that different methods, that were supposed to assess moral values, converged (cf. Campbell \& Fiske, 1959). Secondly, the relationship between implicit and explicit moral values was only established for personal moral values, but not for perceived societal expectations. This finding sheds further light on the meaning of moral prototypes. A culture-specific moral prototype seems to measure what individuals in a culture regard as being morally relevant to themselves. Societal expectations on the other hand seem to measure something else than just the 'collective wisdom' about moral values. It may be that moral prototypes and personal moral values reflect an ideal conception of what is right and wrong. Benevolence, universalism and conformity were here the value types that ranked highest. They relate to the welfare of close and distant others and to self-restraint (Schwartz, 1992). They can be interpreted as typical ideal moral values that facilitate coordinated social interactions. Societal expectations on the other hand may reflect the actual normative pressure about how to behave and what to value, which is considerably different to the ideal conception of morality as often described in the literature.

\footnotetext{
${ }^{29}$ For the actual rank orders of values see Appendix D.
} 
Table 6.7: Spearman correlations between implicit and explicit measures of values.

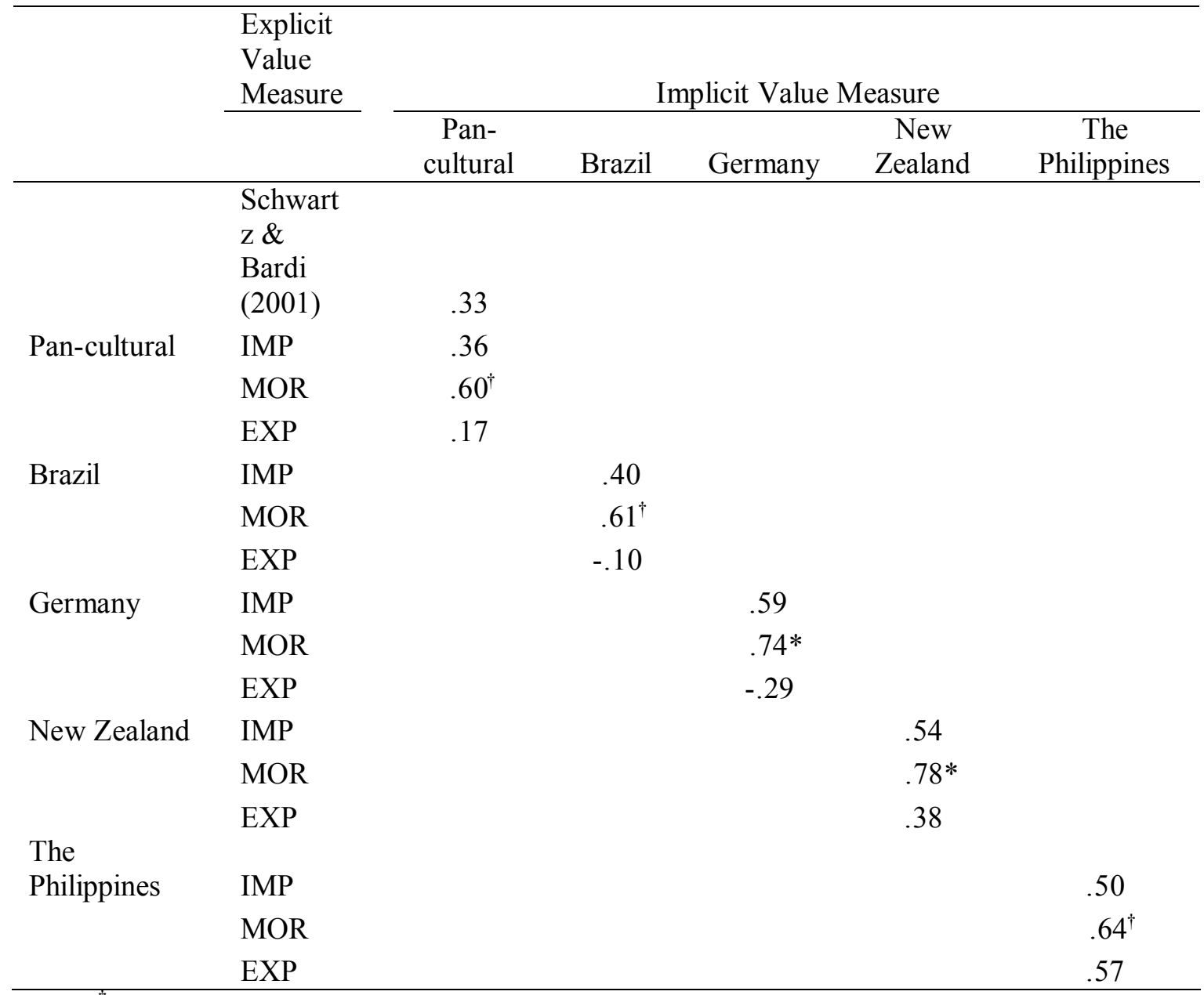

Note. ${ }^{~} p<.10,{ }^{*} p<.05$, two-tailed, $\mathrm{N}=9$ value types. IMP $=$ value hierarchy based on the personal importance rating scale, $\mathrm{MOR}=$ value hierarchy based on the personal moral relevance rating scale, EXP

$=$ value hierarchy based on the societal expectation rating scale.

Personally important value hierarchies differ from personal moral value hierarchies in the way that self-focused values, such as self-direction, seem to play a subordinate role in the latter.

\section{Comparing Features of the 'Moral Person' and the 'Good Person'}

Finally, whether the target for studying moral prototypes is interchangeable with the concept of a 'good person' was examined. Responses by New Zealand students on the 'good' and 'moral' target were compared with each other. Furthermore, Filipino responses from this study using the 'moral' target were compared to data from Smith, et al. (2007) in which Filipino students were presented the 'good' target. There was an 
overlap of 23 trait categories produced by the two Filipino samples. The trait categories that emerged only in Smith, et al.'s (2007) Filipino sample were: active, sense of humour, athletic and attractive. A total of 13 moral traits were unique to the 'moral character' target in my Filipino sample (see Table 6.9): good, right, just, obedient, ethical, has standards, humble, disciplined, virtuous, exemplary, determined, unselfish and wise. The Spearman correlation, including zero frequencies, between both lists of attributes was not significant $\left(r_{s}(39)=-.14, p>.05\right)$, but became marginally significant when zero frequencies were excluded $\left(r_{s}(22)=.42, p=.05\right)$.

In the New Zealand sample, the 'good character' descriptors were reduced to 25 trait categories using the judgment rules as described above. There were three attributes of the 'good person' that did not emerge in the moral trait sample: easy-going, fun and open. A total of six moral trait categories were idiosyncratic to the moral target sample: firm, obedient, proud, religious, respected and right. Table 6.8 displays all attributes and their relative frequencies adjusted by sample size. A Spearman correlation between both targets including all zero frequencies showed that there was no significant correlation $\left(r_{s}(33)=.28, p>.05\right)$ between the two rank orderings when zero frequencies were included. However, the correlation was significant when zero frequencies were excluded $\left(r_{s}(24)=.47, p<.05\right)$. 
Table 6.8: Attributes of a 'good' and a 'moral' person free-listed by two New Zealand samples.

\begin{tabular}{lllll}
\hline & New Zealand 'good' & & New Zealand 'moral' & \\
& target & $\%$ & target & $\%$ \\
\hline 1 & friendly & 67 & honest & 67 \\
2 & honest & 56 & good & 24 \\
3 & kind & 46 & kind & 24 \\
4 & fun & 28 & ethical & 22 \\
5 & caring & 27 & trustworthy & 22 \\
6 & reliable & 26 & caring & 21 \\
7 & helpful & 21 & has standards & 20 \\
8 & loyal & 20 & helpful & 19 \\
9 & trustworthy & 18 & friendly & 18 \\
10 & considerate & 17 & right & 18 \\
11 & generous & 17 & strong & 18 \\
12 & sociable & 16 & considerate & 17 \\
13 & ethical & 12 & compassionate & 11 \\
14 & just & 11 & respectful & 11 \\
15 & happy & 10 & proud & 10 \\
16 & open & 10 & reliable & 10 \\
17 & easy-going & 9 & firm & 9 \\
18 & good & 7 & generous & 9 \\
19 & strong & 7 & just & 8 \\
20 & unselfish & 7 & loyal & 8 \\
21 & determined & 6 & obedient & 8 \\
22 & respectful & 6 & unselfish & 8 \\
23 & warm-hearted & 6 & respected & 7 \\
24 & well-mannered & 6 & tolerant & 7 \\
25 & compassionate & 5 & intelligent & 6 \\
26 & tolerant & 4 & religious & 6 \\
27 & has standards & 2 & well-mannered & 1 \\
28 & intelligent & 2 & determined & 0 \\
29 & firm & 0 & sociable & 0 \\
30 & obedient & 0 & happy & 0 \\
31 & proud & 0 & warm-hearted & 0 \\
32 & religious & 0 & easy-going & \\
33 & respected & 0 & fun & \\
34 & right & 0 & open & \\
& & & & 0 \\
\hline
\end{tabular}


Table 6.9: Attributes of a 'good' and a 'moral' person in two Filipino samples.

\begin{tabular}{|c|c|c|c|c|}
\hline & Filipino 'moral' target & $\%$ & $\begin{array}{l}\text { Filipino 'good' target } \\
\text { (Smith, et al., 2007) }\end{array}$ & $\%$ \\
\hline 1 & religious & 66 & honest & 46 \\
\hline 2 & good & 54 & $\operatorname{kind}^{\mathrm{a}}$ & 44 \\
\hline 3 & honest & 40 & caring & 38 \\
\hline 4 & kind & 35 & sense of humour & 34 \\
\hline 5 & respectful & 33 & understanding & 32 \\
\hline 6 & right & 21 & loving & 26 \\
\hline 7 & well-mannered & 21 & considerate & 26 \\
\hline 8 & just & 19 & helpful & 25 \\
\hline 9 & friendly & 17 & friendly & 21 \\
\hline 10 & generous & 13 & generous & 21 \\
\hline 11 & obedient & 13 & sociable & 21 \\
\hline 12 & ethical & 12 & educated $^{\mathrm{b}}$ & 20 \\
\hline 13 & Has standards & 12 & intelligent & 18 \\
\hline 14 & humble & 11 & responsible & 15 \\
\hline 15 & disciplined & 10 & athletic & 15 \\
\hline 16 & loving & 9 & respectful & 14 \\
\hline 17 & virtuous & 9 & attractive & 14 \\
\hline 18 & exemplary & 8 & well-mannered $^{c}$ & 12 \\
\hline 19 & considerate & 7 & religious & 11 \\
\hline 20 & helpful & 7 & trustworthy & 11 \\
\hline 21 & trustworthy & 7 & active & 11 \\
\hline 22 & caring & 6 & patient & 10 \\
\hline 23 & responsible & 6 & compassionate & 9 \\
\hline 24 & determined & 6 & loyal & 9 \\
\hline 25 & unselfish & 6 & positive & 9 \\
\hline 26 & wise & 6 & hardworking & 9 \\
\hline 27 & compassionate & 5 & self-confident ${ }^{\mathrm{d}}$ & 8 \\
\hline 28 & loyal & 5 & good & 0 \\
\hline 29 & understanding & 4 & right & 0 \\
\hline 30 & sociable & 4 & just & 0 \\
\hline 31 & educated & 4 & obedient & 0 \\
\hline 32 & positive & 4 & ethical & 0 \\
\hline 33 & self-confident & 4 & has standards & 0 \\
\hline 34 & intelligent & 2 & humble & 0 \\
\hline 35 & patient & 1 & disciplined & 0 \\
\hline 36 & hardworking & 1 & virtuous & 0 \\
\hline 37 & sense of humour & 0 & exemplary & 0 \\
\hline 38 & athletic & 0 & determined & 0 \\
\hline 39 & attractive & 0 & unselfish & 0 \\
\hline 40 & active & 0 & wise & 0 \\
\hline
\end{tabular}

Note. Smith, et al. (2007) counted some of the features separately which fall here under one and the same trait category: ${ }^{\mathrm{a}}$ is the sum of frequencies for kind and nice, ${ }^{\mathrm{b}}$ is the sum of frequencies for educated and knowledgeable, ${ }^{\mathrm{c}}$ is the sum of frequencies for courteous and well-mannered, ${ }^{\mathrm{d}}$ is the sum of frequencies for assertive and confident. 
To facilitate again the interpretation of features associated with specific samples as well as the comparison of the relative similarity of the samples, a correspondence analysis on all four samples was conducted. The two-dimensional biplot is depicted in Figure 6.4.

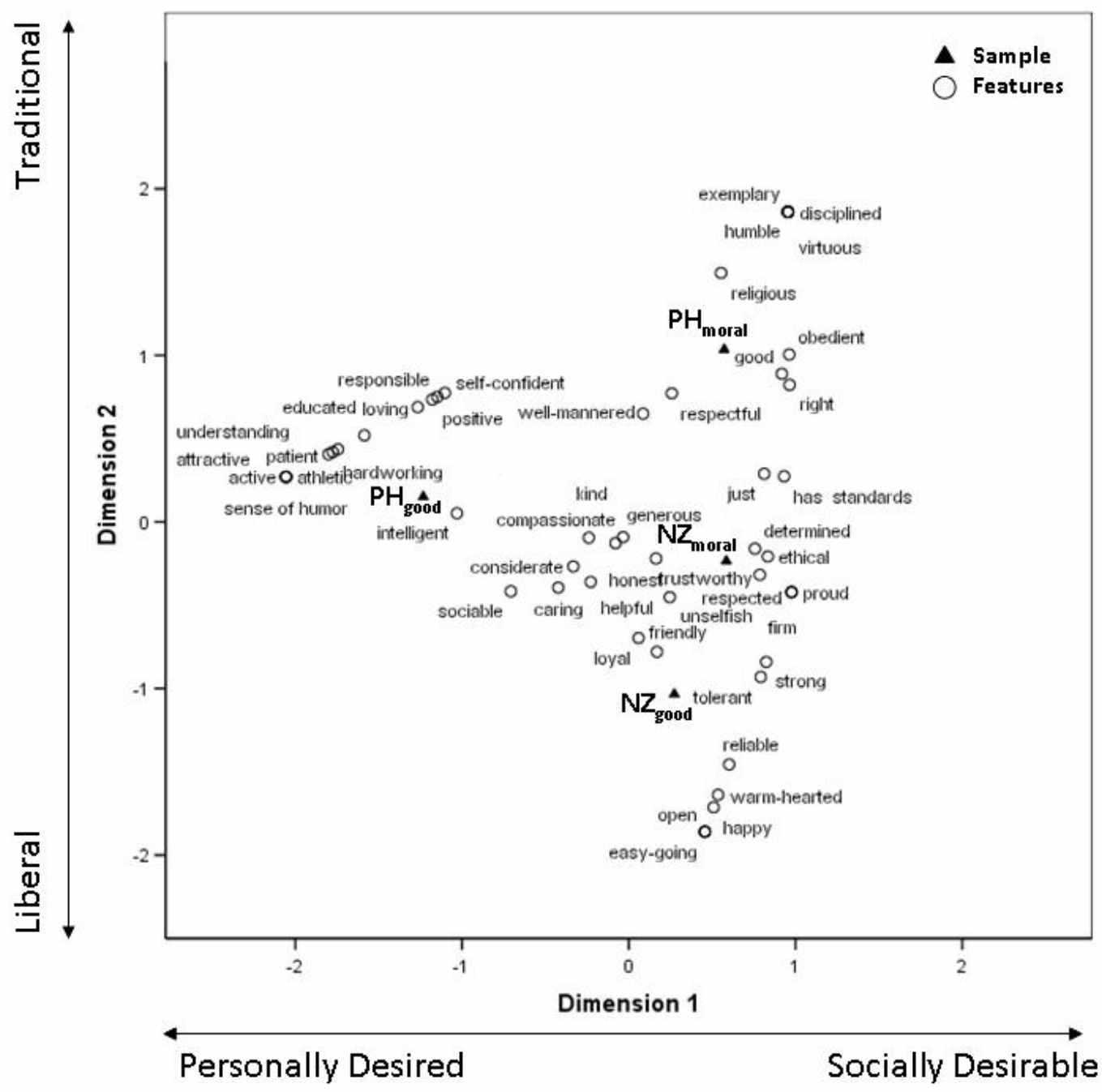

Figure 6.4: Two-dimensional representation of correspondence analysis results for frequencies of 'moral character' and 'good character' trait categories across four samples.

Note. $\mathrm{PH}_{\text {moral }}, \mathrm{PH}_{\text {good, }} \mathrm{NZ}_{\text {moral }}$ and $\mathrm{NZ}_{\text {good }}$ denote samples from the Philippines or New Zealand respectively which received either the 'moral' or 'good' character target. $\mathrm{PH}_{\text {good }}$ is based on data from Smith, et al. (2007). 
The summary statistics produced a significant chi-square $\left(\chi^{2}(150)=1550.43, p\right.$ $<.001)$. The total inertia was high with all dimensions explaining $81.4 \%$ of the variance in the original correspondence table. The first dimension explained $44.1 \%$ of the $81.4 \%$, the second $38 \%$ of it and the third dimension $17.9 \%$ which is lower than the $20 \%$ cutoff recommended by Hair, et al. (2006). Hence, only two dimensions were retained.

An immediately striking feature is that the shape of the points in the map form once more a triangle. The endpoints of this triangle are characterized by the New Zealand 'good target' sample, the Filipino 'good target' sample and the Filipino 'moral target' sample. The New Zealand 'moral target' sample is located close to the origin of the map. Inspection of the sample-specific traits shows that there is again a 'liberaltraditional' distinction characterized by features such as being open-minded, tolerant versus being disciplined and religious. The New Zealand 'good' and 'moral' target samples are both more closely located to the liberal pole (dimension two) whereas the Filipino samples tend to be closer to the traditional pole. In this analysis, it became evident that there were only minor differences between the New Zealand 'good' and 'moral character' target.

The two Filipino samples show a different picture. Their location in the perceptual map shows that they are differently arranged along the first dimension. The interpretation of this dimension is more difficult. Yet, one pole seems to be made up of items that are more about personally desired characteristics (e.g. being attractive and athletic) and the other of socially desirable characteristics (e.g. being just and ethical). Hence, the poles of this dimension may be labelled 'personally desired' versus 'socially desirable'. Interestingly, it appears then that the New Zealand sample yielded traits that are more of the 'liberal' and 'socially desirable' kind regardless of the target. 
Does it matter whether the 'good' or the 'moral' person is used as a target to elicit moral prototypes? The answer seems to be - 'it depends'. It depends whether the samples studied belong to collectivistic or individualistic cultures. The findings suggest that the target does not matter that much when individualistic cultures are studied. However, there may be a considerable difference between the two targets when collectivistic cultures are sampled. The Filipino sample associated with a 'moral person' more traditional traits and values. The Filipino sample with he 'good person' as a target yielded not only what is socially good as opposed to bad, but also what is intellectually good (e.g. being intelligent) and interestingly also a number of characteristics which refer to what is physically good (e.g. being attractive). Hence, in the collectivistic sample the target 'good' yielded a broader category of associations, including what is personally desired, than the target 'moral'.

\section{General Discussion}

\section{Summary}

The present study was designed to answer four central research questions: What kinds of attributes are associated with a 'moral person' (RQ1)? Are there cultural differences and similarities in associations of a 'moral person' (RQ2)? Are implicit and explicit measures of moral values related to each other (RQ3)? Does the 'good person' elicit the same characteristics as the 'moral person' (RQ4)?

In regard to the first research question, I found that the concept of a 'moral person' evokes characteristics which can be arranged along two dimensions in a perceptual map: other- vs. self-focused attributes and traditional vs. liberal attributes. Most of the attributes tended to be located on the 'other-'pole and to imply a 'traditional' connotation. 
Research question two can be answered in the affirmative. There were clear cultural differences in associations regarding a 'moral person'. The Brazilian sample defined the 'traditional' and 'self-focused' dimension in the perceptual map, the Filipino sample the 'traditional' and 'other-focused' dimension, and the German sample the 'other-focused' and 'liberal' dimension. The New Zealand sample was located close to the centroid which means that it shared variance with all the other samples and did not contribute to the definition of any of the dimensions through culturally idiosyncratic attributes. The location of the samples in the map corroborated the expected individualism-collectivism distinction: the Filipino and Brazilian sample were both located at the 'traditional' pole, the German sample at the opposite 'liberal' pole, and the New Zealand sample somewhat in-between.

Regarding research question three, I matched the moral attributes with values from the SVS (Schwartz, 1992). There were a number of trait categories that did not fit with Schwartz' values, such as being friendly, generous, and kind which constitute a set of moral values that is not assessed through the SVS. Furthermore, I found that moral attributes were related to Schwartz' social dimension of his circumplex model. The value types that were most frequently accessed in the free-listing task were benevolence, conformity and universalism which belong to the higher order value orientations of selftranscendence and conservation. I correlated the frequencies of the implicit moral values with explicit ratings of Schwartz' value types from Study 3. I found that only ratings of personal moral values were correlated with frequencies of implicit moral values. This is an interesting finding since it was expected that implicit moral values are a kind of 'collective wisdom' about what is right or wrong and would be related to societal expectation ratings, because the latter reflects culturally determined 'oughts' and 'shoulds'. It seems that what is measured with the implicit method is related to what individuals regard as personally right and wrong. It is not related to what they 
personally regard as important in their lives corroborating the distinction of values as the 'desired' (personal values) and the 'desirable' (moral values). And there is not much cultural variability in the accessibility of moral attributes when they are matched with an expert-designed value inventory. Hence, there is a 'widespread' consensus that a personal morality emphasizes values of benevolence, conformity and universalism.

In regard to research question four whether the targets are interchangeable, it seems that it depends on the cultural sample. In the more collectivistic sample (Filipino sample) the 'moral' target elicited attributes that are defined by "socially desirable" features related to morality. However, the 'good' target elicited attributes that can be described as "personally desired" (e.g. being intelligent, attractive) and that are related to competence and sociability. Hence, the two targets provoked a distinction between 'morality' on the one hand and 'competence' and 'sociability' on the other hand (see also Leach, Ellemers, \& Barreto, 2007, for a distinction of these three concepts). In the more individualistic sample (New Zealand), this distinction did not occur. The 'moral' target was conflated with the 'good' target. It appears that the concept of a good person comprises here the concept of a moral person.

\section{Limitations}

The series of analyses in this study have produced insightful findings in what it means to be a 'moral' or 'good' person in different cultures. When drawing conclusions from the findings, there are also limitations to consider. One of them is the different proportion of females in the samples. The New Zealand sample showed the highest proportion of females which leads to the question whether the responses may not exhibit a gender effect as opposed to a cultural effect. Gender differences in morality have been discussed in moral psychology since Gilligan (1982) argued that women adopt the principle of care in their moral reasoning. However, a careful analysis of the literature 
by Walker (1991) revealed that studies of moral reasoning have generally failed to find gender differences. Nevertheless, it may be that men and women differ in their conception of a 'moral person'. Yet, Walker and Pitts (1998) found that men and women were very similar in their moral prototypicality ratings and concluded that they share a common conception of the 'moral person'. Furthermore, if there was a gender bias in the New Zealand sample, one would expect that certain attributes, such as caring, occurred with a relatively high frequency in the female dominated sample. However, by comparing the two New Zealand samples (moral vs. good target) which differ in their proportion of females ( $71 \%$ versus $59 \%)$, we can see that the presumably 'female' attribute caring occurs almost with the same frequency. Even though, it is not possible to fully exclude gender as an explanation for the emerging New Zealand moral prototype, it seems very unlikely that it has induced a bias.

Another point of consideration is that the cross-cultural comparison of the 'good' and the 'moral' target involves primary data analysis of two New Zealand and a Filipino sample and a secondary data analysis of another Filipino sample provided by Smith, et al. (2007). It is noteworthy that responses of all three samples collected in my study did not differ along the 'personally desired' vs. 'socially desirable' dimension. They only differed in terms of their 'liberal' or 'traditional' orientation. The only sample that defined the 'personally desired' dimension is the one that stems from a secondary data source, i.e. the Filipino sample responding to the 'good person' target. The question is whether this is a genuine result or due to different analytical strategies adopted by the different researchers. A comparison of the content of 'good' and 'moral' Filipino attributes shows that there are indeed very different pattern of responses. What is frequently mentioned in regard to a 'good person' (e.g. sense of humour) is not at all, or not as frequently mentioned in regard to a 'moral person'. Even though Smith et al. (2007) may have used a different categorization scheme, there are such pronounced 
differences in the content of associated attributes that it is unlikely that they are due to analytical differences for deriving the trait categories.

A last point to consider is that no inter-rater reliabilities were obtained for the qualitative analysis. However, the moral trait categories were discussed with another colleague until agreement was achieved. Both approaches have their advantages and disadvantages and they parallel somewhat procedures frequently employed for the translation of surveys: translation-backtranslation and committee approach (cf. Harkness, 2003). Inter-rater reliabilities and translation-backtranslation provide a more objective measure of agreement. At the same time they are also very time consuming and tedious since they need to be conducted by at least two persons separately and the solutions can then be discussed. A group-based analysis, and the committee approach for translating surveys, have the advantage that the problem can be discussed from different perspectives and a good solution can be obtained by critically taking into account the different points of view right from the beginning. Considering that this study produced 692 traits to categorize (after the computerized frequency analysis in Stage 1), it was regarded as more efficient to adopt a group-based analytical approach.

\section{Conclusion}

This study is the first to report a cross-cultural comparison on moral prototypes, using different analytical techniques such as correspondence, content and correlational analysis. The main contributions to the literature are threefold. First, the study offers a detailed account of how qualitative responses can be analyzed to obtain a list of trait categories that are central and peripheral to a prototype. I also presented a more objective criterium (scree plot) to decide which trait categories in a prototype analysis are rather central than peripheral. Since qualitative data analysis is not as structured as 
quantitative analysis, this may be an important step forward for a consistent prototype analysis.

Secondly, the study shows that there are pronounced cultural differences in moral prototypes when laypeople are given the opportunity to free-list their associations of a 'moral person'. It is noteworthy that 'forcing' the free-listed moral attributes into expert-designed categories (here the Schwartz' value types) diminished the differences and rendered the samples more similar to each other. Hence, if cultural differences are the research focus, it is worthwhile to examine laypeople's opinions and beliefs through open-ended responses. This is also consistent with the indigenous psychology movement which often uses qualitative methods (see Kim \& Berry, 1993; Kim, Yang, \& Hwang, 2006, for an introduction into indigenous psychology). Studies examining indigenous notions conceptualized as personality traits, social norms or values are regarded as the most fruitful studies when it comes to the detection of cross-cultural differences (Berry, et al., 2002). Future research in cross-cultural psychology could employ prototype analyses using other concepts than the 'moral person' to uncover cultural differences.

The third main contribution is that the study investigated whether the 'moral person' and the 'good person' produce indeed similar attributes as it has been implicitly claimed in past research (e.g. Lapsley \& Lasky, 2001; Smith, et al., 2007). The findings showed that when the sample is individualistic, it may not make a big difference if the 'good' or the 'moral person' target is used. However, if the research objective is to investigate the moral prototype and the sample is collectivistic-oriented, the 'moral person' should be taken as a target since it produces different attributes compared to the 'good person'. Although this finding warrants further research to confirm its generalizability, it may be an important aspect to consider when conducting crosscultural research on the moral prototype. So far, only one cross-cultural study has been 
conducted with this aim - and this study used the 'good person' as a target (Smith, et al., 2007).

Numerous other researchers used moral traits in their investigation of morality, but did not conduct cultural comparative research so far (e.g. Aquino \& Reed, 2002; Leach, et al., 2007; Narvaez, Lapsley, Hagele, \& Lasky, 2006). Aquino and Reed (2002; Reed \& Aquino, 2003), for instance, developed the moral identity scale which assesses the participants' self-importance of some moral traits. Most of their traits have been identified as central and widely shared among the cultural samples in my research. Nevertheless, some of Aquino and Reed's moral traits (e.g. hardworking) did not emerge as central and shared moral traits in my research. If a cross-cultural study is conducted with this moral identity scale, it would be important to include traits that are 'universal' moral traits to ensure cross-cultural equivalence of this psychometric measure. My research can provide a first indication in this regard. For example, attributes related to Schwartz' (1992) benevolence, conformity and universalism value type would fulfil this requirement.

My results are also highly relevant for future research on moral chronicity. Moral chronicity is defined as the chronic availability of moral knowledge structures (Lapsley \& Lasky, 2001). Narvaez, et al. (2006), for example, argued that the moral personality can be conceptualized in terms of moral chronicity. In order to decide what qualifies as moral knowledge structures, a prototype analysis is conducted beforehand. Lapsley and Lasky (2001) conducted a prototype analysis using the 'good person' as a target in a regional university in the American Midwest. My research showed that the 'good person' and 'moral person' target yield similar attributes in an individualisticoriented sample, but considerably different ones in a collectivistic-oriented sample. Hence, if non-Western samples are studied using the moral chronicity paradigm, I would recommend using the 'moral person' as a target in the prototype analysis to 
distinguish moral chronics from non-chronics in subsequent studies. Future research should be conducted in order to ensure that the differences in targets found across cultural samples show a generalizable trend. 


\section{Introduction}

This final chapter draws a thread through the results of the four empirical studies which aimed to critically examine Schwartz' cultural values concept, and to investigate its relationship to morality. Three of the four studies (Study 2, 3 and 4) were designed to bridge the gap between research on morality on the one hand and values research on the other hand. I have pointed out in the introduction and in chapter two that values are a central concept to the definition and measurement of culture. The common approach in cross-cultural psychology is to ask individuals from different cultures about their personal values and to statistically aggregate the responses at the culture-level, which can be further analyzed with structure-oriented techniques (e.g. factor analysis, MDS). This practice has been criticized as neglecting the external constraints and norms (see e.g. Gelfand, et al., 2006), such as prescriptive beliefs about what people should do or ought to do in a certain culture. Moral psychologists, on the other hand, have not paid much attention to the content of morality, i.e. the moral values that guide people in their lives (e.g. Kohlberg, 1984; Turiel, 1983). The study of cultural differences in moral values has been hindered by the normative epistemological approach to define a priori what belongs in- and outside any definition of morality. Yet, theories proposed by cultural psychologists provide a justification for defining 'moral' in terms of what laypeople judge as moral (Haidt, 2001), and for allocating the cultural variation in these judgments to the domain of morality (Shweder, et al., 1997). These theories also fit with another theoretical perspective in the study of morality (Walker \& Pitts, 1998b): the naturalistic approach focusing on the analysis of laypeople's notions of morality, embedded in common understanding and ordinary language, without imposing philosophical

constraints. 
This thesis touches upon these different research frameworks, and I will outline the contribution made to the literature in these areas, after briefly summarizing the main findings of my studies and pointing out their strengths and limitations. I will finish with some general ideas for future research and practical implications.

\section{Summary of Main Findings}

A schematic overview of the main findings from each study is depicted in Figure 7.1. A common feature of all four studies is that they assessed values at the culturelevel. In culture-level analyses, each nation or ethnic/ cultural group is treated as a single case by aggregating responses from individuals, usually through means (see Study 1, 2, and 3). Study 4, which is based on a prototype analysis, also operates at the culture-level, since the prototype is based on the frequency counts reported in a sample. The main findings of all studies are primarily based on correlational and regression analysis. Three of the four studies have been interconnected with each other: Study 3 includes a replication of the main findings from Study 2; and Study 4 has been used to validate some of the results from Study 3 using a multimethod approach (Campbell \& Fiske, 1959) 


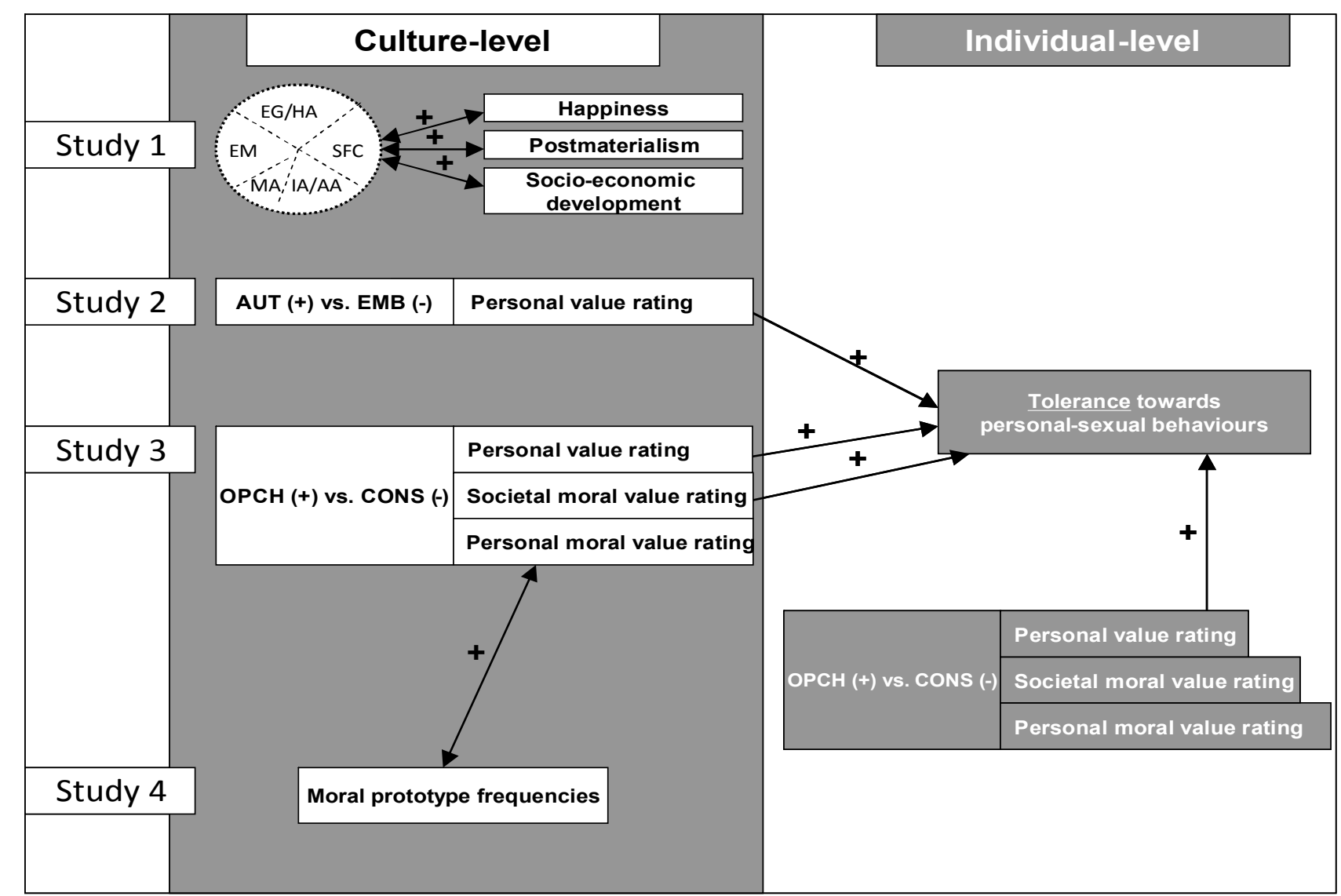

Figure 7.1: Schematic overview of main findings and linkages between studies.

Note. IA $/ \mathrm{AA}=$ Intellectual Autonomy/ Affective Autonomy, MA= Mastery, EM = Embeddedness, EG/ HA = Egalitarianism/ Harmony, SFC $=$ Self-fulfilled Connectedness, AUT $(+)$ vs. EMB $(-)=$ value dimension: Autonomy (positive pole) vs. Embeddedness (negative pole), OPCH (+) vs. CONS (-) = Openness-to-change (positive pole) vs. Conservation (negative pole), + signifies a positive correlation (double arrow) or positive prediction (simple arrow). Tolerance towards personal-sexual behaviours was rated on a $10 \mathrm{pt} \mathrm{Likert}$ scale ranging from never justifiable (1) to always justifiable (10) in Study 2 and on a 5 pt Likert scale ranging from (1) not at all wrong to (5) extremely wrong in Study 3 . The coding of Study 3 has been reversed for the schematic overview so that higher scores represent higher tolerance towards the moral issues. 
The focus in Study 1 was to examine whether Schwartz' $(1994,2006)$ Culturelevel Value Theory is replicable using different samples and a different value inventory. The Rokeach Value Survey (RVS, Rokeach, 1968, 1973, a forerunner of the SVS) was used for this purpose. The RVS is based on a ranking procedure and contains values which match conceptually with half of the items (28 out of 56) of the SVS. A metaanalysis was conducted on the RVS to gather data from samples all around the world. The data were aggregated at the culture-level and value rank orders of 37 cultural groups were analyzed using a multidimensional scaling technique. Qualitative and quantitative comparisons with the SVS value structure corroborated the existence of the main conflicting value types, i.e. Embeddedness vs. Affective/ Intellectual Autonomy and Mastery vs. Egalitarianism/ Harmony, as predicted by Schwartz (1994). Furthermore, some of the Rokeach value items, which were not included in Schwartz' culture-level analysis, were found to form a new value type labelled Self-fulfilled Connectedness (SFC). I proposed that $S F C$ may be an individualistic value orientation that shares some similarity with Autonomy values. However, unlike Autonomy it emphasizes the importance of relationships. Correlations with country indices of happiness, post-materialism and socio-economic development supported the idea that $S F C$ is related to the pursuit of non-material goals and endorsed in countries in which basic needs are fulfilled.

The finding of an additional value cluster is intriguing and it would have been desirable to examine the meaning of the $S F C$ values at the individual-level as well as their link to morality. Time and space constraints made it impossible to do so in this thesis. Nevertheless, there are indications in the literature that SFC, with its self- and other-related value items, is a meaningful value type at the individual-level (see, e.g., Kağıtçıbaşı, 1997, for a theoretical review on the compatibility of autonomy and relatedness). Pohjanheimo (1997), for instance, found an almost identical value type 
when examining the structure of Schwartz' values at the individual-level in a Finnish sample (Helkama, personal communication, November 9, 2009). Furthermore, the literature suggests that the kind of values found in $S F C$ may indeed be related to morality. For example, Waterman (1981) pointed out that the value system of individualism is not necessarily a selfish orientation. He argues that individualism can also be conceptualized as ethical individualism containing a sense of flourishing (eudamonia) and relatedness to others, which parallels the values of SFC. Waterman (1981) suggested that some aspects of ethical individualism underlie what Kohlberg (1969) regards as the highest stage of moral reasoning. Furthermore, it seems that individuals who endorse an ethical kind of individualism are those who can stand up in defiance of the group to defend their principles and engage in political protest activities if necessary (Haan, Smith, \& Block, 1968). Hence, there may be a close relationship between endorsing $S F C$ values and moral reasoning in terms of Kohlberg's principle of justice. There is at least one real-life example that underscores the link between endorsement of $S F C$ values (e.g. happiness, love and harmony) in a subculture and political protests in the name of a universal justice: the hippie movement in the late 1960’s.

Study 2 examined whether cultural values, based on the statistical aggregation of personal values, account for individuals' moral attitudes. The main objective was to test the predictive validity of Schwartz' cultural values in the moral realm and to shed light on the universalism and relativism debate in moral psychology by showing that the answer depends on the moral issues studied. I first conducted a multigroup confirmatory factor analysis on ten items of the Morally Debatable Behaviour Scale (MDBS) supposed to measure different moral attitudes. The data were obtained from the European/ World Value Survey (EVS/ WVS). Metric invariance was confirmed for a two-factor solution (dishonest-illegal and personal-sexual issues) across 80 countries. 
Drawing upon evolutionary and cultural psychological theories, I expected attitudes towards dishonest-illegal behaviours to be independent of cultural values, whereas attitudes towards personal-sexual behaviours would depend on cultural conceptions of the self as either independent or interdependent with others. In two multilevel regression models, Schwartz' cultural values were the independent variables and the dishonestillegal and personal-sexual factor served as dependent variables. After controlling for individual-level differences in moral attitudes as well as the socio-economic development of countries, the findings corroborated that (1) breaches of social cooperation (i.e. dishonest-illegal behaviours) were not related to cultural values, whereas (2) matters of individuals' choices (i.e. personal-sexual behaviours) were judged more strictly in Embeddedness cultures and more leniently in Autonomy-oriented cultures. This study sheds not only light on the universalism and relativism debate, but also on the discriminant validity of cultural values.

Study 1 and 2 showed that Schwartz' cultural values are generalizable and possess predictive validity in the moral realm. The latter contradicts, to some extent, what I have originally argued in my theoretical chapter (chapter two). I scrutinized Schwartz' (1994) measurement of cultural values and argued that it measures what is personally desired instead of what is desirable, the latter consisting of moral beliefs in terms of what is right or wrong and which is intrinsically related to culture (see also Eckensberger, 2007; Miller, 2001). Nevertheless, Study 2 showed that Schwartz' cultural values are consistent with theories of culture and morality concerning the prediction of moral attitudes (Graham, et al., 2009; Shweder, et al., 1997). Hence, it appears that Schwartz' cultural values do assess the general guidelines of what is regarded as right or wrong in a particular culture. Although the finding contradicts my previous argument, it is still explicable. Internalization processes may transform some 
values that are culturally promoted as moral into values that are personally important (cf. Deci \& Ryan, 2000; Ryan \& Deci, 2000).

Nevertheless, at this point, I could have turned away from investigating moral values as an alternative measure of culture. However, there were two reasons why I found it worthwhile to proceed studying moral values as a separate construct. The first reason is conceptual in nature: as with the confusion between values and attitudes which impedes a deeper understanding of the relationship between these two concepts (Rohan, 2000), the failure to distinguish between personal values and moral values may hinder an understanding of how they are related to each other. The second reason is methodological in nature and addresses the common practice in cross-cultural research of measuring cultural values through the statistical aggregation of personal values. There is the problem of causal inconsistency if personal and cultural values are not operationalized differently (Roe \& Ester, 1999): if the assumption is that cultural values influence individuals' values and the aim is to study value change, it becomes difficult to comprehend how a change in cultural values can cause a change in individual values if the change in cultural values is measured through the aggregated changes in individual values. In order to avoid such problems of circularity, it would be necessary to conceptualize and operationalize macro-level constructs differently from micro-level constructs (Liska, 1990). Hence, I designed Study 3 and 4 to measure moral values as a separate construct from personal values and as a valid alternative to Schwartz' cultural values.

In Study 3, I developed a measure of moral values based on my value taxonomy previously put forward in chapter two, in which I distinguished between personal moral values and societal moral values. I expected them to be a valid alternative for the measurement of culture. I constructed a value survey using the SVS in which I modified the response scale in order to measure personal moral values, societal moral values and 
personal values. Data were gathered from more than 1,400 university students from eight countries. I tested the validity of the moral value measures as a macro-level variable for culture by replicating the multi-level regression model from Study 2. Two multilevel regression models using CLOP analyses (James \& Williams, 2000) showed that the following values predicted individuals' moral attitudes after controlling for socio-demographics (age, gender, political orientation, and religiosity) and individuallevel response sets in value ratings: (1) the personal importance of openness-to-change (vs. conservation) at both the individual-and aggregated culture-level was related to a more lenient attitude towards personal-sexual issues, replicating conceptually the crosslevel effect from Study 2; personal moral values did not add incremental validity to this prediction; and (2) the societal expectation of openness-to-change (vs. conservation) at the culture-level was likewise related to a more lenient attitude. However, societal expectation ratings at the individual-level were not reliably related to individuals' moral attitudes, suggesting that it may be a 'true' macro-level concept which operates independently from the micro-level. It seems that a person's (subjective) perception of the societal expectation is not sufficient to have an effect on her moral attitude. Only if there is an (objective) 'agreement' that certain values are expected in the respective society, does it have an effect on a person's attitude towards personal-sexual issues.

Finally, Study 4 was aimed at deriving moral values with an entirely different method. Instead of relying on an 'expert-designed' list of values, the objective was to provide laypeople the opportunity to freely generate moral values by asking them what kind of attributes they associate with a 'moral person'. This is consistent with the naturalistic position in moral psychology. I proposed that these automatic associations could be regarded as an implicit method for deriving moral values. Prototype analysis was used based on counting the occurring frequencies of reported moral attributes, with high frequencies indicating high accessibility and centrality to the prototype. A 
correspondence analysis on the free-listed moral attributes from four cultural samples showed a partition of collectivistic samples reporting more 'traditional' attributes and individualistic samples listing more 'liberal' attributes. When matching the moral trait categories with values from the Schwartz' Value Survey, it became evident that attributes falling into the value types benevolence, conformity and universalism were accessed most in all samples. The moral trait hierarchies were highly correlated across cultural samples when forced into Schwartz' categorization scheme of value types, and were also positively correlated with the value hierarchy produced by personal moral value ratings from Study 3. The latter findings suggest that the moral prototype is associated with a kind of personal morality. This kind of morality tends to be more similar than different across different cultures when matched with the values of the SVS. Hence, Studies 2 and 3 suggest that my theoretical proposition from chapter two needs to be revised: Values assessed as societal expectations exhibit pronounced cultural differences, however, values as assessed as personal moral values tend to be shared across cultures. These studies have provided an important insight into alternative measures of cultural values.

\section{Strengths of this Research}

This thesis has a number of methodological and theoretical strengths. In terms of methodology, very large samples have been used in Study 1 and 2 (minimum of 44,047 respondents and 37 cultural groups), made possible through the use of archival data, meta-analysis and secondary data analyses. Large scale surveys such as the SVS and EVS/ WVS offer a unique opportunity for cross-cultural researchers to test their hypotheses of cultural differences on a more valid basis than a two or three cultural sample approach (cf. van de Vijver \& Leung, 1997). Cross-cultural meta-analyses are hugely beneficial for circumventing large scale primary data collections (cf. 
Norenzayan \& Heine, 2005; van Hemert, 2003) and have already been employed sporadically in cross-cultural research (e.g. Bond \& Smith, 1996; Fischer \& Mansell, in press; Oyserman, et al., 2002; van Hemert, Poortinga, \& van de Vijver, 2007).

Even though primary data collection in a short amount of time is more limited in terms of sampling, a decent sample size was achieved in both Study $3(1,456$ respondents from eight cultures) and Study 4 (560 respondents from four cultures). This is in particular notable for Study 4, which is based on a qualitative method involving open-ended questions that are more time-consuming in their analysis than quantitative data. Nevertheless, the relative big sample size in this study renders the findings more 'trustworthy' - a term used to refer to reliability in qualitative research (Seale, 1999).

Another strength concerns the use of multi-level regression methods in Study 2 and 3. It has been suggested that multilevel modelling is part of a new generation of cross-cultural research methods (Matsumoto \& Yoo, 2006). It comes closer to the reality of culture by taking into account that individuals are nested within cultures. If culture is 'unpackaged' at the societal-level, i.e. decomposed into measurable antecedents (van de Vijver \& Leung, 1997), multilevel modelling is needed to take into account the dependencies that exist between individuals from the same culture. At the same time, this kind of regression modelling also allows testing the effects of culture while statistically controlling for the effect of other non-cultural variables (cf. Matsumoto \& Yoo, 2006). Study 2, for instance, controlled for socio-economic development of countries and socio-demographic characteristics of individuals (see also Study 3) to examine the unique effect of cultural values on the outcome variable (attitudes towards personal-sexual issues). The major strength of this multilevel approach is also that both a micro- and macro-level morality can be simultaneously assessed (here moral values at the individual- and culture-level). This offers a more 
holistic picture, since human morality is commonly seen as a meeting point between the individual and the society.

A further strength of this thesis is the focus on replicating findings. Replication is generally regarded as an important goal for the scientific advancement of psychology (Amir \& Sharon, 1990). Study 1 successfully replicated Schwartz' (1994, 2006) culturelevel theory. In Study 2, I replicated (or cross-validated respectively) the effect of cultural values on individuals' moral attitudes with values derived from a different sample (Schwartz' teacher data). In Study 3, I replicated a main finding from Study 2, i.e. that certain cultural values predict personal-sexual attitudes. In this study all main findings were cross-validated with different individuals and a different set of values (Version 2 of the survey). In Study 4, I successfully triangulated the qualitative findings on an implicit assessment of moral values, with the quantitative findings from Study 3 on an explicit assessment of moral values. I found that implicit moral values from Study 4 correlated only with explicit personal moral value ratings from Study 3. This multimethod validation (Campbell \& Fiske, 1959) clarified that a personal morality is different from a societal morality, and that priming people with the concept of a 'moral person' (Study 4) triggers associations of their personal morality.

At this point, I would also like to emphasize my approach to operationalizing moral values as positive constructs, and not in terms of immoral behaviours or transgressions, as has often been done in the past (Camacho, Higgins, \& Luger, 2003). This approach is also consistent with the recent flourishing of Positive Psychology, and the emphasis that virtues and character strengths are equally important for the understanding of human psychology as the study of psychopathologies (Seligman, Steen, Park, \& Peterson, 2005). The concept of happiness, for example, has been attributed an outstanding role in positive psychology. Happiness is a value that is not contained in the SVS, but that emerged as part of the new individualistic-oriented value 
type SFC in Study 1. From a Positive Psychology perspective, it may be intriguing to further examine $S F C$ as a cultural variable. The cultural emphasis on $S F C$ and happiness values may, for example, have the effect that specific positive and happy aspects in people's life (e.g. a satisfying marital relationship) provide a stronger buffer against depression and suffering in individualistic than in collectivistic countries.

\section{Limitations}

There are a number of limitations to consider when drawing conclusions from this research. One of the major limitations for Study 3 and Study 4 is the use of student samples which are limited in allowing inferences to be drawn about the whole cultural population. Most cross-cultural research relies on university student samples, because it has the advantage that sampling is convenient and somewhat equivalent across cultures (Matsumoto \& Yoo, 2006). Nevertheless, university entry requirements do vary across cultures- for example, in terms of age and also socio-economic status. An age difference across cultural samples was also noticeable in Study 3 and 4. The Filipino sample contained students that were in average relatively young, whereas the German and Brazilian sample were consistently six to seven years older, on average. I paid attention to these issues and controlled for them wherever possible to ensure that between-sample differences were not confounded with non-cultural variables. As it happened age, for example, was not a predictor of moral attitudes in Study 3, contrary to what was found in Study 2 with representative national samples. It appears that the age differences across samples in Study 3 were not big enough to have an effect on the criterion variable. It was not possible to control for all possible variables which may have an effect on the observed cultural differences. For instance, socio-economic status was not assessed in Study 3 and could not be taken into account as a covariate in the regression 
model. Nonetheless, the between-sample effects that were found support the theory and hypotheses, so that the effect of any confounding variables appears to be negligible.

Another point to consider is that, except for Study 1, cultures were treated as equivalent to nations which is a common practice in cross-cultural research (Smith, et al., 2002). This is certainly an oversimplification and it would be desirable to pay more attention to the diversity of cultural and ethnic groups within nations. On the other hand, individuals live in a cultural environment in which the national culture provides a shared source of socialisation and social control (Smith, et al., 2002). Since members of a nation tend to share the same legal and institutional system, it is commonly regarded as justifiable to use nation as a proxy for culture in cross-cultural research. Especially, in Study 3 and 4 the sampled cultural groups are geographically very different, so that between-sample differences are more likely to be due to cultural differences between nations. Relying on nations instead of ethnic groups also helps reducing the complexity of cross-cultural research. For instance, the broad theoretical cultural frameworks and 'maps' that exist so far (e.g. Schwartz, 1993, 2006) mostly focus on nations which make it difficult to employ a theoretical sampling based on ethnic groups. Future research should pay more attention to the issue of ethnic groups within cultures. It would be fascinating to examine whether there are differences between ethnic groups embedded in the same nation. A multilevel modelling could be employed to take into account that ethnic groups are nested within nations. A three-level model is then imaginable in the sense that nation is at the highest level (Level 3), ethnic group at the intermediate level (Level 2) and individuals at the lowest level (Level 1) of analysis. The effects of value orientations on the criterion variable could then be examined at each level. Such a study would also reveal how much variance is explained through values if they are used as predictors at the ethnic group-level or country-level. This would provide some insight into the arguments around whether it is justified to treat nation as a proxy for culture. 
This is certainly a difficult endeavour that may open up even more questions than answers, for example, how to classify and define ethnic groups. To start with, archival data may be very useful, such as data from the EVS/ WVS in which respondents have already been classified into different ethnicities.

Another limitation is that I have committed the reverse ecological fallacy (Hofstede, 1980) in Study 3 by using individual-level concepts (openness-to-change versus conservation values) to describe cultures. This was done on purpose and can be justified on two grounds. First, recent research in which the degree of isomorphism between Schwartz' individual- and culture-level structure has been examined, indicates that the cross-level structural differences are not very big (Fischer, Vauclair, et al., in press). Fischer, et al.'s study did not allow drawing clear conclusions whether the structures are similar enough so that they can be used in an interchangeable way. However, further analyses by Fischer and Poortinga (2009) on isomorphism of value structures using personal, social and cultural value systems (assessed with the SVS) led to the conclusion that a single value structure is most parsimonious. There was little empirical evidence that would suggest that individual- and culture-level value structures are different from each other, to the extent that they needed to be treated as distinct. Fischer and Poortinga (2009) concluded that using the same ten value types at both the individual and culture-level would simplify value research without a substantive loss of information. Recent empirical studies have already employed this practice (Lönnqvist, et al., 2009; Verkasalo, et al., 2009).

The second reason for committing the reverse ecological fallacy lay in the opportunity to use the same value types at the individual- and culture-level. In this way it was possible to test and directly compare the influence of values at both levels of analysis. Study 3 has shown that the effects of personal importance ratings for the value orientation openness-to-change versus conservation were the same at the individual- 
and culture-level. What Study 3 then empirically suggests for the first time is that the individual- and culture-level structure may indeed be so similar that they do not have a different effect when it comes to the prediction of other variables. However, this finding would need to be replicated in future research using other criterion variables, so that it can be claimed as generalizable to psychological domains other than morality. The distinction of levels of analysis is still vexing and hard to comprehend, but my research may stimulate future studies to empirically shed light on the usefulness of this distinction.

A last point to consider is that I focused mainly on the Schwartz' value theory in order to investigate moral values in this thesis. Even though Schwartz' theory is one of the most important and widely used in contemporary values research, there are a number of other value taxonomies and theories that are worthwhile to explore in terms of their relation to morality. Depending on the aims and goals of the research, indigenous value theories (e.g., Enriquez, 1990), domain-specific value taxonomies (see e.g., De Clercq, Fontaine, \& Anseel, 2008, for the organizational context) or even classification of character strengths and virtues (e.g., Peterson \& Seligman, 2004) can be used for this purpose.

\section{Contribution to the Literature}

\section{Cross-cultural Psychology}

Value orientations are commonly regarded as crucial variables for the comparison of cultures (Zavalloni, 1980). It is not surprising that most of the current models of cultural variation in cross-cultural psychology are based on analyses of value differences (cf. Smith \& Schwartz, 1997). How values have been measured in the past can be distinguished into two approaches (see also Harding \& Phillips, 1986): the measurement of (1) values as expressions of personal goals or ideals, preferred or 
desired by the individual, and (2) values as an expression of the preferable, or what 'ought' to be desired, as a matter of conscience. The first approach has a strong tradition and has been employed, for example, by Rokeach (1973) and continued by Schwartz (1992). The second approach has often been acknowledged in value definitions but, as I discussed in the introduction, has rarely been implemented in empirical value research. Some researchers investigating moral values have tended to take the first approach, and claimed that some values are moral values without conceptual justification (e.g. Shen \& Yuan, 1999; Sikula \& Costa, 1994). Hence, moral evaluations of values and personal preferences have not been differentiated in the past, possibly because researchers assumed that what is personally important is also morally relevant. However, whether this is the case is an empirical question to which there has been so far no satisfactory answer (Braithwaite \& Scott, 1991).

Another related controversy in cross-cultural psychology is that, with survey methods, cultural values are determined by the use of aggregated responses on personal values obtained from a sample of individuals and expressed through average statistics. The underlying assumption is that the individual is an informant who can provide information about a group's values when speaking about his own (Zavalloni, 1980), but this alleged conceptual isomorphism between the individual and the group is an oversimplification. It may be misleading to assume that general cultural values are also the personal values held by an individual (Smith, 1969).

\section{Value Measurement}

In her review on definitions of values, Rohan (2000) warned against the inconsistencies in the way values have been defined in the social sciences. The aim of my research was not to add a 'new construct' under the definition of values. In fact, what I have examined here (values as the desirable) and the way I have examined it, is 
new empirically, but long established theoretically (e.g. Kluckhohn, 1952; Parsons \& Shils, 1952). My aim has rather been to use the theoretical evidence and to develop a way of measuring moral values in order to test the utility of this construct as an alternative to conventional measures of cultural values. The contribution of this research to cross-cultural psychology lies in the operationalization of culture as an intrapsychic construct by using the concept of moral values. The collective nature of culture was obtained by combining the responses of individuals from a cultural sample. The difference to previous approaches lies in the fact that 'culture' as an external constraint is already assessed at the individual-level (here as a perceived societal expectation). Correlational and MDS analyses on cultural value hierarchies showed that there were stronger cultural differences when assessing societal moral values as opposed to personal moral values (Study 3). Hence, I would not recommend studying personal moral values if the focus is on examining cultural differences. Personal moral values may be examined in future research if the focus is on individual differences in moral values and their link to other individual-level variables such as moral attitudes or moral behaviour. I showed, for instance, in Study 3 that personal values mediate the relationship between personal moral values and moral attitudes at the individual-level. Future research is needed to confirm that this phenomenon is generalizable to other criterion variables than moral attitudes, such as moral behaviour. I would recommend using Self-Determination Theory (Ryan \& Deci, 2000) which is as a promising framework for this kind of research, since it proposes different stages of value internalization which may explain conceptual differences between personal values and personal moral values.

My analyses in Study 3 also showed that personal values were a significant predictor at both the individual- and culture-level. In contrast, societal moral values were a significant predictor of individuals' moral attitudes at the culture-level, but not 
reliably at the individual-level, which suggests that it might be a purely culture-level construct. What this study can suggest for future research is that societal moral values at the culture-level seem to be a valid macro-level alternative to personal importance ratings. This may be beneficial if the distinction of micro- and macro-level variables is a desirable one, as for example in the study of value change.

\section{Individualism-Collectivism and Values}

The cultural construct of individualism-collectivism, or autonomyembeddedness respectively, plays an important role in my research. I used it to theoretically sample cultural groups, so that differences between individualistic and collectivistic samples were hypothesized and could be examined. My findings corroborated this distinction: Studies 3 and 4 showed a partition of individualistic and collectivistic samples in a perceptual map. Furthermore, Study 2 demonstrated that only values tapping into the individualism-collectivism distinction were significant predictors of individuals' moral attitudes towards personal-sexual issues. This effect was replicated in Study 3 with conceptually related values (openness to changeconservation). Last not least, Autonomy-Embeddedness emerged as a very robust culture-level dimension in Study 1.

In sum, individualism and collectivism seems to be a very strong cultural construct and it is not surprising that it has dominated cross-cultural theory and research over the past 15 years (Matsumoto \& Yoo, 2006). Nevertheless, it has also been heavily criticized for being defined too broadly and inclusively so that it loses its explanatory power. A concept that explains everything can be said to explain nothing (cf. Kağıtçıbaşı, 1997; Poortinga \& Van Hemert, 2001). I have purposely examined the discriminant validity of Autonomy/ Embeddedness in Study 2. Based on evolutionary and cultural theory, I expected that Autonomy/ Embeddedness would not be related to 
moral attitudes towards cheating behaviours, but only to attitudes towards personalsexual matters. The results corroborated the expectation and is an important contribution to the literature showing that the cultural construct of individualism-collectivism cannot explain everything, but has its limitations at least in the moral realm with the items used in this study.

\section{Individualism-Collectivism and Morality}

The individualism-collectivism distinction is also very salient in cultural theories of morality. Shweder, et al. (1997) proposed the ethics of Autonomy and Community which parallels the individualism-collectivism, or Autonomy-Embeddedness, distinction. Although they suggest an additional ethic of Divinity, it seems that it falls under the ethics of Community, since they are both based on obligations, duties, obedience and respect for authorities (Haidt, et al., 1993). Recently, Haidt (2008; see also Graham, et al., 2009) proposed that there are two different kinds of moral systems that suppress selfishness: the individualizing and the binding system. In the individualizing system, cultures try to suppress selfishness by protecting individuals directly (e.g. through the legal system). This approach focuses on the 'individual' as the locus of moral value. Other cultures try to suppress selfishness by strengthening groups and institutions and by binding individuals into roles, duties, and social obligations. Here, the 'group' is the locus of moral value. Even though Haidt does not explicitly refer to individualismcollectivism, by grounding his theory on the individualizing-binding dichotomy he implicitly acknowledges the importance of the individualism-collectivism distinction in morality. My studies (Study 2 and 3) support Haidt's (2008) and Shweder, et al.'s (1997) theories showing that a cultural emphasis on Autonomy (the individualizing system) is related to more tolerance towards behaviours that concern individuals' choices, whereas Embeddedness (the binding system) is related to a stricter attitude 
towards these behaviours because individuals may take into account the consequences of their choices in the context of social obligations and duties to others. These studies contribute to the literature by empirically relating culture and morality. Although theorists have already pointed out that culture and morality are inextricably related to each other (e.g. Miller, 2001), only a few studies have shown this link using quantitative methods. One of them is recent research conducted by Guerra (2008; Guerra \& GinerSorolla, submitted). Guerra developed a survey on the basis of Shweder, et al.'s (1997) ethnographic theory. Future research could relate moral discourses, as measured by Guerra, to moral values. It would be insightful to find out what explains moral behaviour across cultures - a person's moral values or her moral reasoning or both?

\section{Beyond Individualism-Collectivism?}

Although individualism-collectivism played an important role in my research, I also discovered something new that may add to our understanding of this cultural construct. I found that individualism, in the way Schwartz $(1994,2006)$ defined and operationalized it, may lack a relational component. An additional culture-level value type Self-fulfilled Connectedness (SFC) emerged in Study 1, which is closely related to Autonomy and may therefore be regarded as an additional facet to Schwartz' Affective and Intellectual Autonomy. Although this does not suggest a value dimension that is entirely different from the Autonomy-Embeddedness dimension, it is still a promising discovery for our understanding of individualism as it indicates a transcending of selfishness and the relatedness to others (see also Waterman, 1981). Individualism and collectivism are regarded as multidimensional constructs (Berry, et al., 2002) and SFC broadens the concept of individualism by including relationships and the pursuit of nonmaterial goals. It also integrates aspects of another important societal value orientation which is postmaterialism (Inglehart, 2006). Hence, $S F C$ is not a new value orientation 
beyond individualism-collectivism, but it adds an important aspect to individualism as a cultural value orientation, which has so far been neglected. Therefore, it provides a more holistic picture of individualism.

\section{Moral Psychology}

Kohlberg's (1984) stage theory of moral reasoning dominated research in moral psychology for more than two decades. He doubted the usefulness of studying the content of morality in the form of moral values or moral traits and virtues, since one person's integrity is another person's stubbornness and one person's honesty is another person's insensitivity to the feelings of others (Kohlberg \& Mayer, 1972, p. 479). Nevertheless, more recently researchers have pointed out that moral reasoning and the exclusive focus on structure and not content of morality is not sufficient for a comprehensive understanding of moral functioning (e.g. Frimer \& Walker, 2008; Krebs \& Denton, 2005; Walker \& Pitts, 1998b). There is a call for an integrated, multi-level model of moral functioning that includes not only a 'micro-level morality', i.e. face-toface relations or individual differences in morality (Rest, Narvaez, Bebeau, \& Thoma, 1999), but also a macro-level morality including important elements such as culture (cf. Reed \& Stoermer, 2008). It is an ambitious endeavour and this thesis can only contribute pieces to it. Nonetheless, by envisaging a multi-level model through which morality can be explained with individual- and culture-level variables, this research has contributed to a more integrated understanding of morality and its content in form of values.

\section{Investigating Morality from a Descriptive Approach}

A number of theorists in moral psychology have pointed out that moral values may have an important influence on moral functioning and moral behaviour (Blasi, 
1980; Rest, 1984). What is missing so far is a more detailed account of this concrete content (Biel, et al., 1997), especially when it comes to values as defined in the social psychological literature in terms of a conception of the desirable (e.g. Kluckhohn, 1952; Schwartz, 1992) represented by instrumental and terminal values (Rokeach, 1973). The gap in the literature in regard to moral values may be due to the controversy of how to define morality. In the normative approach, moral values are predefined by deriving them from philosophical reasoning, and hence there is only a limited class of values that justify to be called moral values (for instance, justice, fairness; e.g. Kohlberg, 1984; Turiel, 1983). However, philosophically-driven notions of what is morality lead to a conceptual skew in the field, which can be circumvented by focusing on laypeople's conceptions and understanding (Walker \& Hennig, 2004). Hence, in the descriptive approach to morality, moral values can be inferred from laypeople's understanding of right and wrong which allows broadening the concept of moral values from issues of justice to social conventions and expectations (e.g. Haidt, 2001; Shweder, et al., 1997; Walker \& Pitts, 1998b).

I adopted the descriptive approach in my thesis and used the social intuitionism model (Haidt, 2001) as a suitable theoretical framework for the study of moral values. This framework suggests that studying laypeople's intuitive evaluations of right and wrong is a valid approach in investigating morality and moral functioning. I applied this framework in Study 3 by asking individuals to intuitively evaluate which values are morally relevant. The social intuitionism model also corresponds to recent propositions in the literature suggesting the adoption of a more naturalistic conception of morality rooted in everyday language and common understandings. Within this approach, morality can be studied through moral traits and prototype analyses which I implemented in Study 4 (Lapsley \& Lasky, 2001; Walker \& Hennig, 2004; Walker \& Pitts, 1998a). My research has contributed to these recent theoretical developments in 
moral psychology by studying moral values as a special group of values which laypeople experience as prescriptive.

\section{Moral Values}

I examined two kinds of prescriptive values: (1) personal moral values, which may reflect an individual's personal morality as well as the internalized moral values of society, and (2) societal moral values which are the culturally based ideals and expectations set for oneself or for others about how people should live their lives. To the best of my knowledge, this distinction has empirically not been made before. I reasoned that personal moral values can theoretically be in accordance with the moral values of the wider society; however, in individual cases personal moral values may also be distinct from the shared moral values of the collective, because society is not the only source of moral value education (cf. Bandura, 1991). What I empirically found in Study 3 was that personal moral values tended to reflect cultural similarities rather than differences. There was a consensus between individualistic and collectivistic samples, showing that the top ranking personal moral values were benevolence, universalism and conformity. In contrast, there were pronounced cultural differences in terms of societal moral values. The top ranking societal moral values implied a focus on the self (e.g. achievement) in individualistic-oriented samples, whereas in collectivistic samples they tended to be focused on others (e.g. conformity). Furthermore, societal moral value ratings were not correlated with implicit moral values from Study 4, but personal moral value ratings were. This corroborates that personal moral values and societal moral values are different constructs.

To the best of my knowledge, these two studies take a novel approach in examining which of the Schwartz' values are moral values in a variety of different cultures. Furthermore, Study 4 is the first to investigate cross-culturally a moral 
prototype using the 'moral person' as a target for eliciting associations. The finding that achievement is a societal moral value is especially intriguing. Graham, et al. (2009) pointed out that the Schwartz' value type of achievement does neither fit with their Moral Foundation Theory nor with Shweder et al.'s (1997) big three theory on morality. Nevertheless, they suggested that achievement may point to an additional psychological mechanism that supports a moral system. There is also theoretical support for that from a model proposed very recently by Janoff-Bulman, Sheikh and Baldacci (2008). They crossed the approach-avoidance distinction from motivation theory with a self- versus other-focus resulting in four moral motives, of which one is the self-reliance motive. This motive is an approach-based orientation that involves providing for the self. The focus is on activation for one's own advancement and it entails industriousness and independence. The authors point out that this motive may not appear to be related to morality, yet it is akin to a highly valued moral conception in Western culture, the Protestant ethic, with its emphasis on autonomy and hardwork. Further research is needed to examine the link between achievement in individualistic cultures and morality in more detail. Janoff-Bulman et al.'s moral motive of self-reliance, and even McClelland's (1961) research into achievement orientation, may be helpful.

Moral Universalism versus Relativism. It appears that the findings for personal moral values and societal moral values are related to the controversy of whether morality is universal or relative. Evolutionary psychologists and cultural psychologists studying human morality have long suggested that people's moral values are dictated partly by universal moral laws and in part by culturally specific processes (e.g. Haidt, 2001; Haidt \& Joseph, 2004; Krebs, 2008). Boyd and Richerson (1985), for instance, proposed the Dual Inheritance Theory which suggests that human behaviour is shaped by both biological and socio-cultural inheritance systems with their own selective mechanisms and forms of transmissions. Biological evolution operates under the 
premise of natural selection through which a certain genetic trait enhances the probability of survival. Therefore, it is more likely to be transmitted to successive generations through reproduction. This can eventually change the population's genetic makeup regarding this specific trait. It has been proposed that social cooperation has significantly enhanced human survival and hence transmission of genes. In the cultural inheritance paradigm it is argued that values, beliefs and practices of cultures develop in response to environmental needs, and fulfil important social functions such as group stability. Cultural information per se is not transmitted genetically, but through social learning, based on the principles of observation and imitation and with consequences for the whole social group. Therefore, universal values, which prevent any form of harm doing and enhance social cooperation, have been crucial for human survival in the ancestral environment (cf. Krebs, 2008). Culturally specific values on the other hand fulfil the function to adapt to the respective social and physical environments. This may have produced a wide array of different norms and values depending on the demands of the respective ecological context (cf. Krebs, 2004).

The findings from Study 3 (and to some extent also from Study 4) showed that personal moral values are more 'universal' than culturally different, which may be explained with the universal code of cooperation requiring taking care for close others (i.e. prosocial action, benevolence), resolving value conflicts (i.e. exerting justice, universalism) as well as respecting the social order (i.e. controlling antisocial action, conformity, cf. Helkama, 2004). Nevertheless, individualistic and collectivistic cultures may also have developed their own values as an adaptation to the respective environment producing different societal moral values with a greater focus on the individual in individualistic cultures and the social group in collectivistic cultures. Study 2 showed a similar finding: moral attitudes related to breaches of social cooperation were not predicted by cultural values and were therefore judged as wrong 
independently of the cultural context as measured through Schwartz' cultural values. On the other hand, moral attitudes related to personal choices in regard to life and death matters (personal-sexual issues) were predicted by cultural values with a focus on the self (Autonomy) versus others (Embeddedness). In sum, this shows that notions of right and wrong do indeed tell us something about culture, but it depends on (1) the issues studied (personal-sexual issues), and (2) the kind of values measured (societal moral values).

Tentative Explanations. An examination of human history would be desirable at this point to arrive at an explanation for the development of different societal moral values. Yet, such an endeavour is often speculative and leaves some questions unanswered - such as the exact mechanisms underlying cultural differences that persist for centuries or even millennia (Mesoudi, 2007). A tentative explanation may be that societies are characterized by different social structures in which individuals may be tightly linked with one another or alternatively see themselves as independent from each other (see Berry's, 1976, ecocultural model; cf. Moghaddam, et al., 2000; Triandis, 1989). Agricultural societies where the whole family is involved in the production process can be taken as an example of a social group with strong interdependencies between their members. This may have led to the development of a societal moral code that is more based on focusing towards others than on focusing towards the self. The industrial revolution in Western countries may have played an important role in changing the social structure from interdependencies to independence.

Moghaddam et al. (2000) argue that individuals in modern industrial societies still have a sense of duty. Duty may just have another meaning to them by reinterpreting duties towards others into duties towards themselves. Hence, my finding that 'achievement' (i.e. being successful) is a salient societal moral value in the individualistic sample may be explained in terms of a personal duty in individualistic- 
oriented cultures. Being successful is possibly part of a general 'social contract' to participate and contribute to society when individuals are rather independent from each other, and social control is not achieved through the interconnectedness with others as in collectivistic-oriented cultures.

\section{Suggestions for Future Research}

Since morality refers ultimately to moral action (cf. Blasi, 1980), future research could examine moral behaviour and the role of moral values as the motivational component (see also Rest, 1984). It has been suggested that individuals may not act according to the moral principles they claim to value, because of a lack of integrity or self-control (e.g. Bandura, Caprara, Barbaranelli, Pastorelli, \& Regalia, 2001; see also Baumeister \& Exline, 2000). Consider, for example, people who claim to value the environment and who consider 'protection of the environment' to be a moral value. Many people would still take their cars to go to work, even if they could take the bus, their bikes or even just walk. Self-control, i.e. the ability to overcome selfish goals (Baumeister \& Exline, 2000), may be a mediator between evaluating a value as morally relevant and acting according to it. This research would have great practical implications in terms of how to achieve behavioural change such as saving energy. The appeal that protecting the environment is a moral obligation may not be sufficient, but would need to be complemented with an appeal on selfish concerns, for example, that taking the stairs instead of the lift is a good daily exercise to keep oneself fit.

Another valuable area of research would be the study of value fit between the individual and the cultural environment. By operationalizing different values for the macro- and micro-level, as it has been done in this thesis, insight can be gained into individuals' difficulties in adapting to new cultures or into the phenomenon of cultural estrangement. For instance, Bernard, Gebauer and Maio (2006) measured personal 
values and perceived social values (important to British society’s values) and found that discrepancies in these values predicted estrangement, but were not related to subjective well-being. Discrepancies between personal and societal values may be more pronounced when the latter is operationalized in moral terms, such as the perceived societal expectation. Societal expectations are more prescriptive and assess more directly the 'cultural press' a person is exposed to. Since morality regulates what is regarded as right or wrong in a society, violations of prescriptive beliefs may result in sanctions ranging from a raised eyebrow to overt hostility. An applied example can be provided with research conducted by Cortazzi and Jin (1997), who noted that Chinese and British students have different expectations about student and teacher roles. British students and teachers regard the teacher as a facilitator who promotes autonomy. To Chinese students, however, the teacher is a transmitter of knowledge and a role model. Hence, Chinese students tend to participate passively in the classroom, whereas British teachers would expect them to participate actively and to think critically and independently. This mismatch of expectations may be expressed in sanctions in the sense that Chinese students in Britain could receive worse grades because they are perceived as 'lacking' active participation or showing insufficient independent thinking in their coursework. Differences in expectations are especially difficult to deal with if a person's own moral values and those of society are very different. It may finally result in cultural estrangement or marginalization in the case of sojourners. Bernard et al. (2006) called for research investigating the cross-cultural effects of value discrepancies. I believe that assessing the societal expectations herein would be beneficial and could possibly also show that there is indeed a relationship between value discrepancies and subjective well-being.

The investigation of moral values in rhetorics would also be worthwhile. Bain, Kashima and Haslam (2006) pointed out that values are a common tool of mass 
influence in the public domain, but there is surprisingly little research in social psychology that has investigated the role of values in rhetorics. For example, 'moral values' have been regarded as people's main motivation to vote for President Bush in the 2004 presidential election in the United States (Skitka \& Bauman, 2008). It would be interesting to see whether political conservatives use the term 'moral values' more often in political speeches than liberals. Graham et al. (2009) found that there were important differences in the understanding of morality between political liberals and conservatives. Liberals showed greater endorsement of the principles harm/ care and fairness/ reciprocity when it came to moral issues. In contrast, conservatives endorsed additionally the principles of ingroup/ loyalty, authority/ respect and purity/ sanctity. It is possible that conservatives, whose understanding of morality is apparently broader, use the term 'moral' or 'moral values' more often in political speeches than liberals.

It would also be interesting to look at the effect of current economic factors and the relation to moral attitudes. I showed in Study 2 that low human development significantly predicted a stricter attitude towards issues such as abortion and homosexuality. It would be insightful to find out whether the current global economic crisis is reflected in a greater rejection of these kinds of issues. This would help explaining the dynamic underpinnings in the formation of such attitudes.

Last but not least, what should or could be studied in order to understand culture depends on how culture is defined (Toomela, 2003). If culture is defined as a rule system organizing social life which posits boundaries on what is accepted and tolerated, then Kluckhohn's (1952) suggestion could be implemented which would also provide an insight into the moral values of a culture. He stated that social approval and disapproval is manifested in the form of gossip: "Where gossip is most current is where that culture is most heavily laden with values" (p. 404). Such a study could be implemented through qualitative interviews or diaries in order to collect detailed 
information about events that triggered gossip in people's daily lives. Alternatively, educational material could be analyzed for moral values such as school books or even soap operas on TV (see also Kilby, 1993). Based on my findings, I would expect that self-direction and achievement are more emphasized in soap operas in individualistic countries, whereas conformity is more salient in collectivistic ones. There are many research opportunities in this area which have not been fully exhausted yet and it would be insightful to examine whether the findings converge. If so, a more generalizable picture of culture and macro-morality could be obtained.

\section{Practical Implications}

I believe that we have to [...] treat values and moral obligations as a [...] human problem - the most important of all human problems.

\section{- Albert Einstein}

Morality is about organizing social life and getting along with each other. I will not make normative claims on the basis of my research findings, since this would mean committing the naturalistic fallacy (Moore, 1903) which occurs when what 'is' is equated with what 'should be'. Hence, I will not claim that striving for certain values is better than others. Nevertheless, I see some important practical implications in my findings. There were clear cultural differences in societal expectations. Individualisticoriented samples yielded that their society is perceived as achievement-oriented. On the other hand, collectivistic-oriented samples showed that they perceived their society to be more conformity-oriented. Knowing about these kinds of fundamental differences can be very important in intercultural encounters, especially when it is in situations in which performance is central such as at the workplace or at school or university.

I also found that people from different cultures agree on certain moral values. People seem to commonly acknowledge that being nice to others (benevolence), being just (universalism) and maintaining the social order (conformity) are important moral 
values. This may provide a foundation upon which moral dialogue can be based on. Schwartz and Bardi (2000) have already drawn parallels between values and moral discourse. They found that benevolence, self-direction and universalism were the most widely shared personal values, and they argued that these values may provide a common moral ground. I agree with Schwartz and Bardi (2000) that shared values may enable a worldwide moral dialogue. Nevertheless, my findings suggest that conformity may be more accepted as a shared moral value than self-direction. My findings would also support the universality of Habermas' (2005) Theory of Communicative Rationality specifying how to engage in moral discourse. He proposes that there are conditions under which the discourse must take place, meaning that people need to accept certain ground rules (the value of conformity): all men are to be treated equal (the value of universalism) and participants must be truthful and try to mutually understand each other (the value of benevolence). Only if these requirements are fulfilled, ethical truth can be achieved. Emphasizing this common understanding of right and wrong may provide the ground for discourse and rapprochement beyond cultural differences.

\section{Final Conclusion}

The task of developing research on values that may capture the dialectical relationship between the individual and the environment remains formidable.

- Marisa Zavalloni (1980, p. 76)

I have investigated morality as a mental phenomenon that consists of values and attributes that can be judged as right or wrong. I expected that people from different cultures and societies prioritize these traits and values in different ways. My final goal was to show that moral values can be taken as an alternative to the conventional measurement of cultural values derived from aggregated personal values. It has been a 
difficult endeavour which is certainly related to the abstract nature of the moral values concept, but also to the interface of individual and society that is implied in morality. Nevertheless, there are two main conclusions that can be pointed out. First, my research has shown that cultural variability in moral values can be measured by asking for societal expectations. Secondly, I also found that one of the challenges in the study of morality, which lies in the central question whether morality is universal or relative (Miller, 2001), cannot be answered in absolute terms. I found that whether the answer to the question What is right? What is wrong? tells something about culture depends (1) on the issue (personal-sexual issues), and (2) on the kind of values studied (societal moral values). Hence, the moral universalism/ relativism debate is not black or white, and warrants further research in order to understand the grey area. There are still a number of open questions such as: How do individuals negotiate their personal morality with the perceived societal expectations? How can cultures with different societal moral principles (e.g. self-direction versus tradition) come to a common ground? These exemplary questions are intriguing to research and of great societal importance. It would be of great value to see more studies in cross-cultural psychology that devote their focus on a micro- and macro-morality to answer these kinds of questions. 


\section{REFERENCES}

Aijzen, I. (1991). The theory of planned behavior. Organizational Behavior and Human Decision Processes, 50, 179-211.

Allport, G. W. (1955). Becoming: Basic considerations for a psychology of personality. New Haven, CT: Yale University Press.

Amir, Y., \& Sharon, I. (1990). Replication research: A "Must" For the scientific advancement of psychology. Journal of Social Behavior and Personality, Special Issue, 5, 51-69.

Amodio, D. M., Devine, P. G., \& Harmon-Jones, E. (2007). A dynamic model of guilt: Implications for motivation and self-regulation in the context of prejudice. Psychological Science, 18, 524-530.

Aquino, K., \& Reed, A., II (2002). The self-importance of moral identity. Journal of Personality and Social Psychology 83, 1423-1440.

Axelrod, R. (1984). The evolution of cooperation. New York: Basic Books.

Axelrod, R., \& Hamilton, W. D. (1981). The evolution of cooperation. Science, 211, 1390-1396.

Baer, D., Curtis, J., Grabb, E., \& Johnston, W. (1996). What values do people prefer in children? A comparative analysis of survey evidence from fifteen countries. In C. Seligman, J. M. Olson \& M. P. Zanna (Eds.), The psychology of values: The ontario symposium (Vol. 8, pp. 299-328). Hillsdale, NJ, England: Lawrence Erlbaum Associates.

Bain, P. G., Kashima, Y., \& Haslam, N. (2006). Conceptual beliefs about human values and their implications: Human nature beliefs predict value importance, value trade-offs, and responses to value-laden rhetoric. Journal of Personality and Social Psychology, 91, 351-367.

Baldwin, J. R., Faulkner, S. L., Hecht, M. L., \& Lindsley, S. L. (2006). Redefining culture: Perspectives across the disciplines. Mahwah, NJ, US: Lawrence Erlbaum Associates Publishers.

Bandura, A. (1991). Social cognitive theory of moral thought and action. In W. M. Kurtines \& J. L. Gewirtz (Eds.), Handbook of moral behavior and development: Theory, research and applications (pp. 71-129). Hillsdale, NJ: Erlbaum.

Bandura, A., Caprara, G. V., Barbaranelli, C., Pastorelli, C., \& Regalia, C. (2001). Sociocognitive self-regulatory mechanisms governing transgressive behavior. Journal of Personality and Social Psychology, 80, 125-135.

Bardi, A., \& Schwartz, S. H. (2003). Values and behavior: Strength and structure of relations. Personality and Social Psychology Bulletin, 29, 1207-1220.

Batson, C. D., Engel, C. L., \& Fridell, S. R. (1999). Value judgments: Testing the somatic-marker hypothesis using false physiological feedback. Personality and Social Psychology Bulletin, 25, 1021-1032.

Baumeister, R. F., \& Exline, J. J. (2000). Self-control, morality, and human strength. Journal of Social \& Clinical Psychology. Special Issue: Classical Sources of Human Strength: A Psychological Analysis, 19, 29-42.

Baumeister, R. F., Stillwell, A. M., \& Heatherton, T. F. (1994). Guilt: An interpersonal approach. Psychological Bulletin, 115, 243-267.

Bedford, O., \& Hwang, K.-K. (2003). Guilt and shame in Chinese culture: A crosscultural framework from the perspective of morality and identity. Journal for the Theory of Social Behaviour, 33, 127-144.

Bentler, P. M. (1992a). Comparative fit indexes in structural models. Psychological Bulletin, 112, 400-404. 
Bentler, P. M. (1992b). On the fit of models to covariances and methodology to the bulletin. Psychological Bulletin, 112, 400-404.

Bernard, M. M., Gebauer, J. E., \& Maio, G. R. (2006). Cultural estrangement: The role of personal and societal value discrepancies. Personality and Social Psychology Bulletin, 32, 78-92.

Berry, J. W. (1976). Human ecology and cognitive style: Comparative studies in cultural and psychological adaptation. New York: Sage/ Halsted/Wiley.

Berry, J. W., Poortinga, Y. H., Segall, M. H., \& Dasen, P. R. (2002). Cross-cultural psychology: Research and applications (2 ed.). Cambridge: University Press.

Biel, A., Fransson, N., \& Dahlstrand, U. (1997). Moral values assigned to real-life events. Scandinavian Journal of Psychology, 38, 21-28.

Bilsky, W., \& Schwartz, S. H. (1994). Values and personality. European Journal of Personality, 8, 163-181.

Blasi, A. (1980). Bridging moral cognition and moral action: A critical review of the literature. Psychological Bulletin, 88, 1-45.

Bond, M. H. (2002). Reclaiming the individual from Hofstede's ecological analysis- a 20-year odyssey: Comment on Oyserman et al. (2002). Psychological Bulletin, $128,73-77$.

Bond, M. H., Leung, K., Au, A., Tong, K.-K., de Carrasquel, S. R., Murakami, F., et al. (2004). Culture-level dimensions of social axioms and their correlates across 41 cultures. Journal of Cross-Cultural Psychology, 35, 548-570.

Bond, M. H., \& Pang, M. K. (1991). Trusting the tao: Chinese values and the recentering of psychology. Bulletin of the Hong Kong Psychological Society, 26/27, 5-27.

Bond, R., \& Smith, P. B. (1996). Culture and conformity: A meta-analysis of studies using Asch's (1952b, 1956) line judgment task. Psychological Bulletin, 119, 111-137.

Borg, I., \& Groenen, P. J. F. (1997). Modern multidimensional scaling. Theory and applications. New York Springer-Verlag.

Bovasso, G., Jacobs, J., \& Rettig, S. (1991). Changes in moral values over three decades, 1958-1988. Youth and Society, 22, 468-481.

Braithwaite, V. A., \& Scott, W. A. (1991). Values. In J. P. Robinson, P. R. Shaver \& L. S. Wrightsman (Eds.), Measures of personality and social psychological attitudes (Vol. 1, pp. 661-753). San Diego, California: Academic Press, Inc.

Brewer, M. B., \& Chen, Y.-R. (2007). Where (who) are collectives in collectivism? Toward conceptual clarification of individualism and collectivism. Psychological Review, 114, 133-151.

Briggs, S. R., \& Cheek, J. M. (1986). The role of factor analysis in the development and evaluation of personality scales. Journal of Personality, 54, 106-148.

Brökelmann, S., Fuchs, C.-M., Kammhuber, S., \& Thomas, A. (2005). Beruflich in Brasilien [Professionally in Brazil]. Göttingen: Vandenhoek \& Ruprecht.

Broom, D. M. (2003). The evolution of morality and religion. Cambridge: Cambridge University Press.

Brotemarkle, R. A. (1922). A comparison test for investigating the ideational content of the moral concepts. Journal of Applied Psychology, 6, 235-242.

Browne, M. W., \& Cudeck, R. (1992). Alternative ways of assessing model fit. Sociological Methods \& Research, 21, 230-258.

Bruner, J. S. (1990). Acts of meaning. Cambridge, MA: Harvard University Press.

Camacho, C. J., Higgins, E. T., \& Luger, L. (2003). Moral value transfer from regulatory fit: What feels right is right and what feels wrong is wrong. Journal of Personality and Social Psychology, 84, 498-510. 
Campbell, D. T. (1986). Science's social system of validity-enhancing collective believe change and the problems of the social sciences. In D. W. Fiske \& R. A. Shweder (Eds.), Metatheory in social science (pp. 108-135). Chicago: University of Chicago Press.

Campbell, D. T., \& Fiske, D. W. (1959). Convergent and discriminant validation by the multitrait-multimethod matrix. Psychological Bulletin, 56, 81-105.

Campbell, R. L., Christopher, J. C., \& Bickhard, M. H. (2002). Self and values: An interactivist foundation for moral development. Theory \& Psychology, 12, 795822.

Cattell, R. B. (1966). The scree test for the number of factors. Multivariate Behavioral Research, 1, 245-276.

Chen, F. F. (2008). What happens if we compare chopsticks with forks? The impact of making inappropriate comparisons in cross-cultural research. Journal of Personality and Social Psychology, 95, 1005-1018.

Cheung, G. W., \& Rensvold, R. B. (2000). Assessing extreme and acquiescence response sets in cross-cultural research using structural equations modeling. Journal of Cross-Cultural Psychology, 31, 187-212.

Cheung, G. W., \& Rensvold, R. B. (2002). Evaluating goodness-of-fit indexes for testing measurement invariance. Structural Equation Modeling, 9, 233-255.

Chinese Culture Connection (1987). Chinese values and the search for culture-free dimensions of culture: The Chinese Culture Connection. Journal of CrossCultural Psychology, 18, 143-164.

Chiu, C., Dweck, C. S., Tong, J. Y., \& Fu, J. H. (1997). Implicit theories and conceptions of morality. Journal of Personality and Social Psychology, 73, 923940.

Chiu, C., \& Hong, Y. (1997). Justice from a Chinese perspective. In H. S. R. Kao \& D. Sinha (Eds.), Asian perspectives on psychology (pp. 164-184). Thousand Oaks, CA: Sage.

Cialdini, R. B., Reno, R. R., \& Kallgren, C. A. (1990). A focus theory of normative conduct: Recycling the concept of norms to reduce littering in public places. Journal of Personality and Social Psychology, 58, 1015-1026.

Commandeur, J. J. F. (1991). Matching configurations. Leiden, The Netherlands: DSWO Press.

Consalvi, C. (1971). Some cross- and intracultural comparisons of expressed values of Arab and American college students. Journal of Cross-Cultural Psychology, 2, 95-107.

Cortazzi, M., \& Jin, L. (1997). Communication for learning across cultures. In D. McNamara \& R. Harris (Eds.), Overseas students in higher education (pp. 7690). London: Routledge.

Cortina, J. M. (1993). What is coefficient alpha? An examination of theory and applications. Journal of Applied Psychology, 78, 98-104.

Cosmides, L., \& Tooby, J. (1992). Cognitive adaptations for social exchange. In J. H. Barkow, L. Cosmides \& J. Tooby (Eds.), The adapted mind: Evolutionary psychology and the generation of culture (pp. 163-228). New York, NY: Oxford University Press.

Crissman, P. (1942). Temporal change and sexual difference in moral judgments. Journal of Social Psychology, 16, 29-38.

Cronbach, L. J. (1951). Coefficient alpha and the internal structure of tests. Psychometrika, 31, 93-96.

D'Andrade, R. G. (1984). Cultural meaning systems. In R. A. Shweder \& R. LeVine (Eds.), Culture theory: Essays on mind, self, and emotion (pp. 88-119). Cambridge: Cambridge University Press. 
Darwin, C. (1874). The descent of man and selection in relation to sex. New York: Rand, McNally \& Company.

Dawkins, R. (1976). The selfish gene. Oxford: Oxford University Press.

De Clercq, S., Fontaine, J. R. J., \& Anseel, F. (2008). In search of a comprehensive value model for assessing supplementary person organization fit. Journal of Psychology, 142, 277-302.

De Houwer, J., \& Teige-Mocigemba, S. (2009). Implicit measures: A normative analysis and review. Psychological Bulletin, 135, 347-368.

De Munck, V. C. (2001). In the belly of the beast: Two incomplete theories of culture and why they dominate the social sciences (part 2). Cross-Cultural Psychology Bulletin, 35, 5-17.

de Waal, F. (1996). Good natured: The origins of right and wrong in humans and other animals. London: Harvard University Press.

Deci, E. L., \& Ryan, R. M. (1995). Human autonomy: The basis for true self-esteem. In M. Kemis (Ed.), Efficacy, agency, and self-esteem (pp. 31-49). New York: Plenum.

Deci, E. L., \& Ryan, R. M. (2000). The "what" and "why" of goal pursuits: Human needs and the self-determination of behavior. Psychological Inquiry, 11, 227268.

Dekker, S., \& Fischer, R. (2008). Cultural differences in academic motivation goals: A meta-analysis across thirteen societies. Journal of Educational Research, 102, 99-110.

Dewey, J. (1939). The theory of valuation. Chicago: University of Chicago Press.

Diener, E., Diener, M., \& Diener, C. (1995). Factors predicting the subjective wellbeing of nations. Journal of Personality and Social Psychology, 69, 851 -864.

Diessner, R., Mayton, D., \& Dolen, M. A. (1993). Values hierarchies and moral reasoning. Journal of Social Psychology, 133, 869-871.

Durkheim, E. (1893/1984). The division of labour in society (W. D. Halls, Trans.). London: Macmillan.

Dworkin, R. (1978). Taking rights seriously. Cambridge, MA: Harvard University Press.

Earley, P. C. (2006). Leading cultural research in the future: A matter of paradigms and taste. Journal of International Business Studies, 37, 922-931.

Earley, P. C., \& Ang, S. (2003). Cultural intelligence: Individual interactions across cultures. Stanford University Press: Palo Alto, CA.

Earley, P. C., \& Mosakowski, E. (2002). Linking culture and behavior in organizations: Suggestions for theory development and research methodology. In F. Dansereau \& F. J. Yammarino (Eds.), Research in multi-level issues: The many faces of multi-level issues (Vol. 1, pp. 279-319). San Francisco: Elsevier Science.

Eckensberger, L. H. (2007). Morality from a cultural psychology perspective. In G. Zheng, K. Leung \& J. G. Adair (Eds.), Perspectives and progress in comtemporary cross-cultural psychology (pp. 25-34). Beijing: China Light Industry Press.

Eisenberg, N. (2000). Emotion, regulation and moral development. Annual Review of Psychology, 51, 665-697.

Enriquez, V. G. (1990). Indigenous personality theory. In V. G. Enriquez (Ed.), Indigenous psychology: A book of readings. (pp. 285-308). Quezon City: Akademya Ng Sikoholhiyang Pilipino.

Feather, N. T. (1985). Values in education and society. New York: Free Press.

Feather, N. T. (1996). Values, deservingness, and attitudes toward high achievers: Research on tall poppies. In C. Seligman, J. M. Olson \& M. P. Zanna (Eds.), The 
ontario symposium: The psychology of values (Vol. 8, pp. 215-251). Mahwah, NJ: Lawrence Erlbaum Associates, Inc.

Feather, N. T. (1999). Values, achievement, and justice: Studies in the psychology of deservingness. New York: Kluwer Academic/ Plenum Publishers.

Fehr, B. (1988). Prototype analysis of the concepts of love and commitment. Journal of Personality and Social Psychology, 55, 557-579.

Fehr, B., \& Russell, J. A. (1984). Concept of emotion viewed from a prototype perspective. Journal of Experimental Psychology: General, 113, 464-486.

Festinger, L. (1957). A theory of cognitive dissonance. Stanford, CA: Stanford University Press.

Figueroa, J. G., González, E. G., \& Solís, V. M. (1976). An approach to the problem of meaning: Semantic networks. Journal of Psycholinguistic Research, 5, 107-115.

Fischer, R. (2006). Congruence and functions of personal and cultural values: Do my values reflect my culture's values? Personality and Social Psychology Bulletin, $32,1419-1431$.

Fischer, R. (2008a). Multilevel approaches in organizational settings: Opportunities, challenges and implications for cross-cultural research. In F. J. R. v. d. Vijver, D. A. v. Hemert \& Y. H. Poortinga (Eds.), Individuals and cultures in multilevel analysis (pp. 173-196). Mahwah, NJ: Lawrence Erlbaum Associates.

Fischer, R. (2008b). Rewarding seniority: Exploring cultural and organizational predictors of seniority allocations. Journal of Social Psychology, 148, 167-186.

Fischer, R., \& Chalmers, A. (2008). Is optimism universal? A meta-analytical investigation of optimism levels across 22 nations. Personality and Individual Differences, 45, 378-382.

Fischer, R., Fontaine, J. R. J., van de Vijver, F., \& van Hemert, D. (in press). What is style and what is bias in cross-cultural comparisons? An examination of response styles in cross-cultural research. In A. Gari \& K. Mylonas (Eds.), Quod erat demonstrandum: From herodotus' ethnographic journeys to cross-cultural research. Athens: Pedio.

Fischer, R., \& Mansell, A. (in press). Commitment across cultures: A meta-analytical approach. Journal of International Business Studies.

Fischer, R., \& Poortinga, Y. H. (2009). An examination of similarities in personal, social and cultural value structures.Unpublished manuscript, Victoria University of Wellington, Wellington.

Fischer, R., Smith, P. B., Richey, B. E., Ferreira, M. C., Assmar, E. M. L., Maes, J., et al. (2007). Organizational reward allocation principles: Testing organizational and cross-cultural differences. Journal of Cross-Cultural Psychology, 38, 1-16.

Fischer, R., Vauclair, C.-M., Fontaine, J. R. J., \& Schwartz, S. H. (in press). Are individual- and culture-level value structures different? Testing Hofstede's legacy with Schwartz' data. Journal of Cross-Cultural Psychology.

Fishbein, M., \& Ajzen, I. (1975). Belief, attitude, intention, and behavior: An introduction to theory and research. Reading, Mass.: Addison-Wesley Pub.

Flanagan, J. (1954). The critical incident technique. Psychological Bulletin, 51, $327-$ 358.

Fontaine, J. R. J. (1999). Culturele vertekening in schwartz' waardeninstrument: Een exemplarische onderzoek naar culturele vertekening in sociaal-psychologische en persoonlijkheidsvragenlijsten [cultural bias in schwartz's value instrument: An exemplary study into cultural bias in social-psychological and personality questionnaires]. Unpublished doctoral dissertation, Katholieke Universiteit Leuven, Leuven, Belgium.

Fontaine, J. R. J. (2008). Traditional and multilevel approaches in cross-cultural research: An integration of methodological frameworks. In F. J. R. van de 
Vijver, D. A. van Hemert \& Y. H. Poortinga (Eds.), Individuals and cultures in multi-level analysis (pp. 65-92). Mahwah, NJ: Lawrence Erlbaum Associates.

Fontaine, J. R. J., \& Fischer, R. (in press). Multilevel structural equivalence. In D. Matsumoto \& F. Van de Vijver (Eds.), Handbook of cross-cultural research methods. Oxford: University Press.

Fontaine, J. R. J., Poortinga, Y. H., Delbeke, L., \& Schwartz, S. H. (2008). Structural equivalence of the values domain across cultures: Distinguishing sampling fluctuations from meaningful variation. Journal of Cross-Cultural Psychology, 39, 345-365.

Fontaine, J. R. J., Poortinga, Y. H., Setiadi, B., \& Markam, S. (2002). Cognitive structure of emotion terms in Indonesia and the Netherlands. Cognition and Emotion, 16, 61-86.

Freedom House (no date). Annual survey of freedom country scores 1972-1973 to 1998-1999. Retrieved from http://www.freedomhouse.org/rankings.pdf, January 2008.

Frimer, J. A., \& Walker, L. J. (2008). Towards a new paradigm of moral personhood. Journal of Moral Education, 37, 333 - 356.

Fu, J. H., \& Chiu, C. (2007). Local culture's responses to globalization: Exemplary persons and their attendant values. Journal of Cross-Cultural Psychology, 38, 636-653.

Fuchs, C.-M. (2004). Identifikation brasilianischer Kulturstandards im Handlungsfeld deutscher Fach- und Führungskräfte [Identification of the main cultural differences between Germans and Brazilians at the workplace]. Unpublished Master thesis, University of Regensburg, Regensburg.

Fuchs, C.-M. (2005). Arbeitsplatz Globus - kulturelle Unterschiede als "Störvariable" [Workplace globe - cultural differences as "confounding variable"]. In H. Stubbe \& C. d. Santos-Stubbe (Eds.), Kölner Beiträge zur Ethnopsychologie und transkulturellen Psychologie (Vol. 6, pp. 41-72). Göttingen: Vandenhoek \& Ruprecht.

Fuchs, C.-M. (2006). Brasilianische Spielregeln [Brazilian rules of the game]. In D. Studienpreis (Ed.), Mythos markt? Die ökonomische, rechtliche und soziale Gestaltung der Arbeitswelt [Myth market? The economical, legal, and social creation of the work environment] (pp. 231-252). Wiesbaden: VS Verlag für Sozialwissenschaften.

Gabrenya, W. (1999). Psychological anthropology and the "levels of analysis" problem: We married the wrong cousins. In J. C. Lasry, J. Adair \& K. Dion (Eds.), Latest contributions to cross-cultural psychology (pp. 333-351). Lisse, the Netherlands: Swets \& Zeitlinger.

Gelfand, M. J., Nishii, L. H., \& Raver, J. L. (2006). On the nature and importance of cultural tightness-looseness. Journal of Applied Psychology, 91, 1225-1244.

Gewirth, A. (1984). Ethics. Encyclopaedia britannica (Vol. 6, pp. 976-998). Chicago: Encyclopaedia Britannica.

Gilligan, C. (1982). In a different voice: Psychological theory and women's development. Cambridge, MA, US: Harvard University Press.

Gorsuch, R. L., \& Barnes, M. L. (1973). Stages of ethical reasoning and moral norms of Carib youths. Journal of Cross-Cultural Psychology, 4, 283-301.

Gouldner, A. W. (1960). The norm of reciprocity: A preliminary statement. American Sociological Review. Special Issue: Looking forward, looking back: Continuity and change at the turn of the millenium, 25, 161-178.

Gouveia, V. V., \& Ros, M. (2000). Hofstede and Schwartz's models for classifying individualism at the cultural level: Their relation to macro-social and macroeconomic variables. Psicothema, 12, 25-33. 
Graham, J., Haidt, J., \& Nosek, B. A. (2009). Liberals and conservatives rely on different sets of moral foundations. Journal of Personality and Social Psychology, 96, 1029-1046.

Graham, J. W. (2009). Missing data analysis: Making it work in the real world. Annual Review of Psychology, 60, 549-576.

Greenacre, M. J. (1984). Theory and applications of correspondence analysis. London: Academic Press.

Greene, J. (2007, October 9). The biology of morality: Neuroscientists respond to Killen and Smetana. Human Development, 1, Letters to the Editor. Retrieved from http://www.karger.com/hde/, February 2009.

Greenwald, A. G., \& Banaji, M. R. (1995). Implicit social cognition: Attitudes, selfesteem, and stereotypes. Psychological Review, 109, 3-25.

Gregg, A. P., Hart, C. M., Sedikides, C., \& Kumashiro, M. (2008). Everyday conceptions of modesty: A prototype analysis. Personality and Social Psychology Bulletin, 34, 978-992.

Guerra, V. M. (2008). Community, autonomy, and divinity: Studying morality across cultures. Unpublished Doctoral Dissertation, University of Kent, Kent.

Guerra, V. M., \& Giner-Sorolla, R. (2010). Community, autonomy, and divinity scale (CADS): Development of a theory-based moral codes scale for research across and within cultures. Journal of Cross-cultural Psychology, 41, 35-50.

Haan, N. (1982). Can research on morality be "Scientific"? American Psychologist, 37, 1096-1104

Haan, N., Smith, M. B., \& Block, J. (1968). Moral reasoning of young adults: Politicalsocial behavior, family background, and personality correlates. Journal of Personality and Social Psychology, 10, 183-201.

Habermas, J. (2005). The meaning of moral ought. In W. Edelstein \& G. NunnerWinkler (Eds.), Morality in context (pp. 27-40). Amsterdam, Netherlands: Elsevier.

Haidt, J. (2001). The emotional dog and its rational tail: A social intuitionist approach to moral judgment. Psychological Review, 108, 814-834.

Haidt, J. (2003). The moral emotions. In R. J. Davidson, K. R. Scherer \& H. H. Goldsmith (Eds.), Handbook of affective sciences. Oxford: Oxford University Press.

Haidt, J. (2008). Morality. Perspectives on Psychological Science, 3, 65-72.

Haidt, J., \& Graham, J. (2006). When morality opposes justice: Conservatives have moral intuitions that liberals may not recognize. Social Justice Research, 20, 98116.

Haidt, J., \& Hersh, M. (2001). Sexual morality: The cultures and reasons of liberals and conservatives. Journal of Applied Social Psychology, 31, 191-221.

Haidt, J., \& Joseph, C. (2004). Intuitive ethics: How innately prepared intuitions generate culturally variable virtues. Daedalus, Fall, 55-66.

Haidt, J., \& Joseph, C. (2007). The moral mind: How 5 sets of innate moral intuitions guide the development of many culture-specific virtues, and perhaps even modules. In P. Carruthers, S. Laurence \& S. Stich (Eds.), The innate mind (Vol. 3, pp. 367-392). New York: Oxford University Press.

Haidt, J., Koller, S. H., \& Dias, M. G. (1993). Affect, culture, and morality, or is it wrong to eat your dog? Journal of Personality and Social Psychology, 65, 613628.

Hair, J. F., Jr., Black, W. C., Babin, B. J., Anderson, R. E., \& Tatham, R. L. (2006). Multivariate data analysis. Upper Saddle River, NJ: Pearson Prentice Hall.

Halman, L. (1996). Individualism in individualized society? Results from the European values surveys. International Journal of Comparative Sociology, 37, 195-214. 
Halpern, D. (2001). Moral values, social trust and inequality: Can values explain crime? The British Journal of Criminology, 41, 236.

Hamilton, W. D. (1964). The genetical evolution of social behaviour I and II. Journal of Theoretical Biology, 7, 1-16 and 17-52.

Harding, S., \& Phillips, D. (1986). Contrasting values in Western Europe. Unity, diversity and change. London: Macmillan.

Hardy, S. A., Padilla-Walker, L. M., \& Carlo, G. (2008). Parenting dimensions and adolescents' internalisation of moral values. Journal of Moral Education, 37, 205-223.

Harkness, J. (2003). Questionnaire translation. In J. A. Harkness, F. J. R. van de Vijver \& P. P. Mohler (Eds.), Cross-cultural survey methods (pp. 35-56). New York: Wiley.

Haste, H., \& Abrahams, S. (2008). Morality, culture and the dialogic self: Taking cultural pluralism seriously. Journal of Moral Education, 37, 377 - 394.

Heine, S. J., \& Norenzayan, A. (2006). Toward a psychological science for a cultural species. Perspectives on Psychological Science, 1, 251-269.

Helkama, K. (1982). The development of moral reasoning and moral values. Acta Psychologica Fennica, 9, 99-111.

Helkama, K. (2004). Values, role-taking and empathy in moral development. Nouvelle Revue de Psychologie Sociale/ New Review of Social Psychology, 3, 103-111.

Helkama, K., Uutela, A., Pohjanheimo, E., Salminen, S., Koponen, A., \& RantanenVäntsi, L. (2003). Moral reasoning and values in medical school: A longitudinal study in Finland. Scandinavian Journal of Educational Research, 47, 399-411.

Henry, P. J., \& Reyna, C. (2007). Value judgments: The impact of perceived value violations on American political attitudes. Political Psychology, 28, 273-298.

Herndon Jr., N. C., Fraedrich, J. P., \& Yeh, Q.-J. (2001). An investigation of moral values and the ethical content of the corporate culture: Taiwanese versus U.S. Sales people. Journal of Business Ethics, 30, 73-85.

Higgins, E. T. (2000). Social cognition: Learning about what matters in the social world. European Journal of Social Psychology, 30, 3-39.

Higgins, E. T. (2006). Value from hedonic experience and engagement. Psychological Review, 113, 439-460.

Hills, M. D. (2002). Kluckhohn and Strodtbeck's values orientation theory. Online Readings in Psychology and Culture, Unit 6, Chapter 3. Retrieved from http://www.ac.wwu.edu/ culture/index-cc.htm, April 2006.

Hitlin, S., \& Piliavin, J. A. (2004). Values: Reviving a dormant concept. Annual Review of Sociology, 30, 359-393.

Hofstede, G. (1980). Culture's consequences: International differences in work-related values. Beverly Hills, CA Sage.

Hofstede, G. (1998). A case for comparing apples with oranges: International differences in values. International Journal of Comparative Sociology, 39, 1631 .

Hogan, R., \& Dickstein, E. (1972). A measure of moral values. Journal of Consulting and Clinical Psychology, 39, 210-214.

Horowitz, L. M., \& Turan, B. (2008). Prototypes and personal templates: Collective wisdom and individual differences. Psychological Review, 115, 1054-1068.

Inglehart, R. (1990). Culture shift in advanced industrial society. Princeton: Princeton University Press.

Inglehart, R. (1997). Modernization and postmodernization. Cultural, economic, and political change in 43 countries. Princeton, NJ: Princeton University Press.

Inglehart, R. (2006). Mapping global values. Journal of Comparative Sociology, 5, 115 136. 
Inglehart, R., \& Baker, W. E. (2000). Modernization, cultural change, and the persistence of traditional values. American Sociological Review. Special Issue: Looking forward, looking back: Continuity and change at the turn of the millenium, 65, 19-51.

Inglehart, R., \& Welzel, C. (2005). Modernization, cultural change and democracy. New York and Cambridge: Cambridge University Press.

Jahoda, G. (1984). Do we need a concept of culture? Journal of Cross-Cultural Psychology, 15, 139-151.

James, L. R., \& Williams, L. J. (2000). The cross-level operator in regression, ANCOVA, and contextual analysis. In J. K. Klein \& S. W. J. Kozlowski (Eds.), Multilevel theory, research and methods in organizations: Foundations, extensions, and new directions (pp. 349-381). San Francisco: Jossey-Bass.

Janoff-Bulman, R., Sheikh, S., \& Baldacci, K. G. (2008). Mapping moral motives: Approach, avoidance, and political orientation. Journal of Experimental Social Psychology, 44, 1091-1099.

Janoff-Bulman, R., Sheikh, S., \& Hepp, S. (2009). Proscriptive versus prescriptive morality: Two faces of moral regulation. Journal of Personality and Social Psychology 96, 521-537.

Jensen, L. A. (1995). Habits of the heart revisited: Autonomy, community, and divinity in adults' moral language. Qualitative Sociology, 18, 71-86.

Johnson, T., Kulesa, P., Llc, I., Cho, Y. I., \& Shavitt, S. (2005). The relation between culture and response styles: Evidence from 19 countries. Journal of CrossCultural Psychology, 36, 264-277.

Jose, P. E. (2003). Medgraph: A programme to graphically depict mediation among three variables: The internet version. Retrieved from http://www.vuw.ac.nz/psyc/staff/paul-jose/files/medgraph/medgraph.php, June 2009.

Kağıtçıbaşı, C. (1997). Individualism and collectivism. In J. W. Berry, M. H. Segall \& Ç. Kağıtçıbaşı (Eds.), Handbook of cross-cultural psychology (2nd ed., Vol. 3, pp. 1-49). Boston, MA: Allyn \& Bacon.

Kağıtçıbaşı, Ç. (2005). Autonomy and relatedness in cultural context: Implications for self and family. Journal of Cross-Cultural Psychology, 36, 403-422.

Kant, I. (1994). Ethical philosophy: Grounding for the metaphysics of morals (J. W. Ellington, Trans. 2nd ed.). Indianapolis and Cambridge: Hackett Publishing. (Original work published 1785).

Kaplan, H. B., \& Johnson, R. J. (2001). Social deviance: Testing a general theory. New York: Kluwer Academic/Plenum Publishers.

Kashima, Y. (2000). Conceptions of culture and person for psychology. Journal of Cross-Cultural Psychology, 31, 14-32.

Kearns, J. N., \& Fincham, F. D. (2004). A prototype analysis of forgiveness. Personality and Social Psychology Bulletin, 30, 838-855.

Kilby, R. W. (1993). The study of human values. Lanham, MD, England: University Press of America.

Kilmann, R. H. (1981). Toward a unique/ useful concept of values for interpersonal behavior: A critical review of the literature on value. Psychological Reports, 48, 939-959.

Kim, U., \& Berry, J. W. (1993). Indigenous psychologies: Experience and research in cultural context. Newbury Park, CA: Sage.

Kim, U., Yang, K. S., \& Hwang, K. K. (2006). Indigenous and cultural psychology: Understanding people in context. New York: Springer.

Klein, K. J., Bliese, P. D., Kozlowski, S. W. J., Dansereau, F., Gavin, M. B., Griffin, M. A., et al. (2000). Multi-level analytical techniques: Commonalities, differences 
and continuing questions. In J. K. Klein \& S. W. J. Kozlowski (Eds.), Multilevel theory, research and methods in organizations: Foundations, extensions, and new directions (pp. 512-553). San Francisco: Jossey-Bass.

Kline, R. B. (2004). Replication and meta-analysis. In R. B. Kline (Ed.), Beyond significance testing: Reforming data analysis methods in behavioral research (pp. 247-271). Washington, DC, US: American Psychological Association.

Klinger, M. R. B. (1962). Moral values across cultures. Personnel \& Guidance Journal, 41, 139-143.

Kluckhohn, C. (1952). Values and value-orientations in the theory of action: An exploration in definition and classification. In T. Parsons \& E. Shils (Eds.), Toward a general theory of action (pp. 388-433). Cambridge, MA: Harvard University Press.

Kluckhohn, F. R., \& Strodtbeck, F. L. (1961). Variations in value orientations. Evanston, Ill: Row, Peterson.

Kohlberg, L. (1958). The development of modes of moral thinking and choice in the years 10-16. Unpublished Doctoral dissertation, University of Chicago, Chicago.

Kohlberg, L. (1969). Stage and sequence: The cognitive-developmental approach to socialization. In D. A. Goslin (Ed.), Handbook of socialization theory and research (pp. 347-480). Chicago: Rand Mc-Nally.

Kohlberg, L. (1984). The psychology of moral development: Essays on moral development (Vol. 2). San Francisco, CA: Harper \& Row.

Kohlberg, L., \& Mayer, R. (1972). Development as the aim of education. Harvard Educational Review, 42, 449-496.

Krebs, D. (2005). The evolution of morality. In D. M. Buss (Ed.), The Handbook of EvolutionaryPpsychology (pp. 747-771). Hoboken, NJ, US: John Wiley \& Sons Inc.

Krebs, D. L. (2008). Morality: An evolutionary account. Perspectives on Psychological Science, 3, 149-172.

Krebs, D. L., \& Denton, K. (2005). Toward a more pragmatic approach to morality: A critical evaluation of Kohlberg's model. Psychological Review, 112, 629-649.

Kristiansen, C. M., \& Hotte, A. M. (1996). Morality and the self: Implications for the when and how of value-attitude-behavior relations. In C. Seligman, J. M. Olson \& M. P. Zanna (Eds.), The psychology of values: The ontario symposium (Vol. 8, pp. 77-105). Hillsdale, NJ, England: Lawrence Erlbaum Associates.

Kristiansen, C. M., \& Zanna, M. P. (1994). The rhetorical use of values to justify social and intergroup attitudes. Journal of Social Issues, 50, 47-65.

Kroeber, A. L., \& Kluckhohn, C. (1952). Culture: A critical review of concepts and definitions. Cambridge, Mass.: Peabody Museum of American Archaeology and Ethnology, Harvard University.

Kruskal, J. B., \& Wish, M. (1978). Multidimensional scaling. London: Sage.

Kuckartz, U. (2007). Maxqda 2007 reference manual for Windows 2000 and XP. Marburg, Germany: Verbi Software.

Kuper, A. (1999). Culture - the anthropologists' account. London: Harvard University Press.

Kurtines, W., \& Gewirtz, J. (1984). Morality, moral behavior, and moral development. New York: John Willey \& Sons, Inc.

Lapsley, D. K., \& Hill, P. L. (2008). On dual processing and heuristic approaches to moral cognition. Journal of Moral Education, 37, 313 - 332.

Lapsley, D. K., \& Lasky, B. (2001). Prototypic moral character. Identity: An International Journal of Theory and Research, 1, 345-363. 
Lapsley, D. K., \& Narvaez, D. (Eds.). (2004). Moral development, self, and identity. Mahwah, NJ, US: Lawrence Erlbaum Associates Publishers.

Leach, C. W., Ellemers, N., \& Barreto, M. (2007). Group virtue: The importance of morality (vs. Competence and sociability) in the positive evaluation of ingroups. Journal of Personality and Social Psychology 93, 234-249.

Lehman, D. R., Chiu, C. Y., \& Schaller, M. (2004). Psychology and culture. Annual Review of Psychology, 55, 689-714.

Leung, K., Bond, M. H., Reimel de Carrasquel, S., Muñoz, C., Hernández, M., Murakami, F., et al. (2002). Social axioms: The search for universal dimensions of general beliefs about how the world functions. Journal of Cross-Cultural Psychology, 33, 286-302.

Levitin, T. (1973). Values. In J. P. Robinson \& P. R. Shaver (Eds.), Measures of social psychological attitudes (pp. 489-502). Ann Arbor, MI: Institute for Social Research.

Lewin, K. (1952). Constructs in field theory. In D. Cartwright (Ed.), Field theory in social science: Selected theoretical papers by kurt lewin (pp. 30-42). London: Tavistock.

Lievens, F., De Corte, W., \& Schollaert, E. (2008). A closer look at the frame-ofreference effect in personality scale scores and validity. Journal of Applied Psychology, 93, 268-279.

Lipsey, M. W., \& Wilson, D. B. (2001). Practical meta-analysis. Thousand Oaks, CA: Sage.

Liska, A. E. (1990). The significance of aggregate dependent variables and contextual independent variables for linking macro and micro theories. Social Psychology Quarterly, 53, 292-301.

Lönnqvist, J.-E., Verkasalo, M., Helkama, K., Andreyeva, G. M., Bezmenova, I., Rattazzi, A. M. M., et al. (2009). Self-esteem and values. European Journal of Social Psychology, 39, 40-51.

Maccoby, E. (1968). The development of moral values and behaviour in childhood. In J. A. Clausen (Ed.), Socialisation and society (pp. 227-269). Boston: Little, Brown and Co.

Maddison, A. (no date). World population, GDP and per capita GDP, 1-2003 ad. Retrieved from http://www.ggdc.net/Maddison/Historical_Statistics/horizontalfile 03-2007.xls, January 2008.

Maehr, M., \& Nicholls, J. (1980). Culture and achievement motivation: A second look. In N. Warren (Ed.), Studies in cross-cultural psychology (Vol. 2, pp. 221-267). New York: Academic Press.

Marks, G., \& Miller, N. (1987). Ten years of research on the false-consensus effect: An empirical and theoretical review. Psychological Bulletin, 102, 72-90.

Markus, H. R., \& Hamedani, M. G. (2007). Sociocultural psychology: The dynamic interdependence among self systems and social systems. In S. Kitayama \& D. Cohen (Eds.), Handbook of cultural psychology (pp. 3-39): Guilford Press.

Markus, H. R., \& Kitayama, S. (1991). Culture and the self: Implications for cognition, emotion, and motivation. Psychological Review, 98, 224-253.

Matsumoto, D. (2003). The discrepancy between consensual-level culture and individual-level culture. Culture \& Psychology, 9, 89-95.

Matsumoto, D. (2007). Culture, context, and behavior. Journal of Personality 75, 12861319.

Matsumoto, D., \& Yoo, S. H. (2006). Toward a new generation of cross-cultural research. Perspectives on Psychological Science, 1, 234-250.

McClelland, D. C. (1961). The achieving society. New York: Free Press. 
Meade, A. W., Michels, L. C., \& Lautenschlager, G. J. (2007). Are internet and paperand-pencil personality tests truly comparable?: An experimental design measurement invariance study. Organizational Research Methods, 10, 322-345.

Mesoudi, A. (2007). Using the methods of experimental social psychology to study cultural evolution. Journal of Social, Evolutionary, and Cultural Psychology, 1, $35-58$.

Miller, G. A. (1956). The magical number seven, plus or minus two: Some limits on our capacity for processing information. Psychological Review, 63, 81-97.

Miller, J., Bersoff, D. M., \& Harwood, R. L. (1990). Perceptions of social responsibilities in India and the United States: Moral imperatives or personal decisions? Journal of Personality and Social Psychology, 58, 33-47.

Miller, J. G. (2001). Culture and moral development. In D. Matsumoto (Ed.), The Handbook of Culture and Psychology (pp. 151- 170). Oxford: University Press.

Mogghaddam, F. M., \& Studer, C. (1997). Cross-cultural psychology: The frustrated gadfly's promises, potentialities, and failures. In D. Fox \& I. Prilleltensky (Eds.), Critical psychology: An introduction (pp. 185-201). Thousand Oaks, CA: Sage.

Moghaddam, F., Slocum, N. R., Finkel, N., Tzili, M., \& Harré, R. (2000). Toward a cultural theory of duties. Culture and Psychology, 6, 275 - 302.

Moore, G. E. (1903). Principia ethica. Cambridge: Cambridge University Press.

Moors, G., \& Wennekers, C. (2003). Comparing moral values in Western European countries between 1981 and 1999. A multiple group latent-class factor approach. International Journal of Comparative Sociology, 44, 155-172.

Mueller, D. J., \& Wornhoff, S. A. (1990). Distinguishing personal and social values. Educational and Psychological Measurement, 50, 691-699.

Mueller, K. H. (1958). Theory for campus discipline. Personnel and Guidance Journal, 36, 302-309.

Muthén, B. O. (1994). Multilevel covariance structure analysis. Sociological Methods Research, 22, 376-398.

Narvaez, D., Lapsley, D. K., Hagele, S., \& Lasky, B. (2006). Moral chronicity and social information processing: Tests of a social cognitive approach to the moral personality. Journal of Research in Personality, 40, 966-985.

Neuliep, J. W., \& Crandall, R. (1993). Everyone was wrong: There are lots of replications out there. Journal of Social Behavior \& Personality. Special Issue: Replication research in the social sciences, 8, 1-8.

Nevitte, N., \& Cochrane, C. (2006). Individualization in Europe and America: Connecting religious and moral values. Journal of Comparative Sociology, 5, 203-230.

Nisan, M. (1987). Moral norms and social conventions: A cross-cultural comparison. Developmental Psychology, 23, 719-725.

Norenzayan, A., \& Heine, S. J. (2005). Psychological universals: What are they and how can we know? Psychological Bulletin, 131, 763-784.

Norris, P., \& Inglehart, R. (2004). Sacred and secular. Religion and politics worldwide. New York: Cambridge University Press.

Nucci, L. P., \& Turiel, E. (1978). Social interactions and the development of social concepts in preschool children. Child Development, 49, 400-407.

Nucci, L. P., Turiel, E., \& Encarnacion-Gawrych, G. (1983). Children's social interactions and social concepts: Analyses of morality and convention in the virgin islands. Journal of Cross-Cultural Psychology, 14, 469-487.

Nunnally, J. C. (1978). Psychometric theory (2nd ed.). New York: McGraw-Hill.

Osgood, C. E., Suci, G. J., \& Tannenbaum, P. H. (1957). The measurement of meaning. Chicago: University of Illinois Press. 
Oyserman, D., Coon, H. M., \& Kemmelmeier, M. (2002). Rethinking individualism and collectivism: Evaluation of theoretical assumptions and meta-analyses. Psychological Bulletin 128, 3-72.

Pakizeh, A., Gebauer, J. E., \& Maio, G. R. (2007). Basic human values: Inter-value structure in memory. Journal of Experimental Social Psychology, 43, 458-465.

Parsons, R., \& Shils, E. (1952). Systems of value orientations. In T. Parsons \& E. Shils (Eds.), Toward a general theory of action (pp. 159-189). Cambridge, MA: Harvard University Press.

Paulhus, D. L. (1991). Measurement and control of response bias. In J. P. Robinson, P. R. Shaver \& L. S. Wrightsman (Eds.), Measures of personality and social psychological attitudes (pp. 17-59). New York: Academic Press.

Peterson, C., \& Seligman, M. E. P. (2004). Character strengths and virtues: A handbook and classification. Oxford: Oxford University Press.

Piaget, J. (1932/1965). The moral judgment of the child. Oxford, England: Harcourt, Brace.

Pittel, S. M., \& Mendelsohn, G. A. (1966). Measurement of moral values: A review and critique. Psychological Bulletin, 66, 22-35.

Pohjanheimo, E. (1997). Arvojen muutos, työ ja sosiaalinen tausta. Tutkimus työikäisistä pyhtääläisistä 1982-1993. [Changing values, work and social background: A study of the work-aged at pyhtää 1982-1993]. Unpublished Doctoral thesis, University of Helsinki, Helsinki.

Pojman, L. P. (1995). Ethics: Discovering right and wrong. Belmont, CA: Wadsworth Publishing Company.

Poortinga, Y. H. (2003). Coherence of culture and generalizability of data: Two questionable assumptions in cross-cultural psychology. In V. Murphy-Berman \& J. J. Berman (Eds.), Cross-cultural differences in perspectives on the self (Vol. 49 of the Nebraska symposium on motivation, pp. 274-322). Lincoln, NE, US: University of Nebraska Press.

Poortinga, Y. H., \& Van Hemert, D. A. (2001). Personality and culture demarcating between the common and the unique. Journal of Personality, 69, 1033-1060.

Popenoe, D. (1983). Sociology (5th ed.). Englewood Cliffs, NJ: Prentice-Hall.

Power, F. C. (2004). The moral self in community. In D. K. Lapsley \& D. Narvaez (Eds.), Moral development, self, and identity (pp. 47-64). Mahwah, NJ, US: Lawrence Erlbaum Associates Publishers.

Preacher, K. J., \& Hayes, A. F. (2004). SPSS and SAS procedures for estimating indirect effects in simple mediation models. Behavior Research Methods, Instruments \& Computing, 36, 717-731.

Price, M. E., Cosmides, L., \& Tooby, J. (2002). Punitive sentiment as an anti-free rider psychological device. Evolution and Human Behavior, 23, 203-231.

Quinones-Vidal, E., Lopez-Garcia, J. J., Penaranda-Ortega, M., \& Tortosa-Gil, F. (2004). The nature of social and personality psychology as reflected in jpsp, 1965-2000. Journal of Personality and Social Psychology, 86, 435-452.

Raudenbush, S. W., \& Bryk, A. S. (2002). Hierarchical linear models. Applications and data analysis methods (2nd ed.). Thousand Oaks, CA: Sage Publications.

Rawls, J. (1971). A theory of justice. Cambridge, MA: Harvard University Press.

Rawls, J. (1999). A theory of justice (Rev. ed.). Cambridge, MA: Harvard University Press.

Reed, A., II, \& Aquino, K. F. (2003). Moral identity and the expanding circle of moral regard toward out-groups. Journal of Personality and Social Psychology 84, 1270-1286. 
Reed, D. C., \& Stoermer, R. M. (2008). Towards an integrated model of moral functioning: An overview of the special issue. Journal of Moral Education, 37, $417-428$.

Rest, J., Narvaez, D., Bebeau, M., \& Thoma, S. (1999). A neo-Kohlbergian approach: The DIT and schema theory. Educational Psychology Review, 11, 291-324.

Rest, J. R. (1984). The major components of morality. In W. M. Kurtines \& J. L. Gewirtz (Eds.), Morality, moral behavior, and moral development (pp. 24-38). New York: John Willey \& Sons, Inc.

Richerson, P. J., \& Boyd, R. (2005). Not by genes alone: How culture transformed human evolution. New York: Viking.

Roccas, S., Sagiv, L., Schwartz, S. H., \& Knafo, A. (2002). The big five personality factors and personal values. Personality and Social Psychology Bulletin, 28, 789-801.

Roe, R. A., \& Ester, P. (1999). Values and work: Empirical findings and theoretical perspective. Applied Psychology: An International Review. Special Issue: Values and work, 48, 1-21.

Rohan, M. J. (2000). A rose by any name? The values construct. Personality and Social Psychology Review, 4, 255-277.

Rohan, M. J., \& Maiden, W. (2000). Investigations into “value fit.".Unpublished manuscript, University of New South Wales, Sydney.

Rohan, M. J., \& Zanna, M. P. (1998). The "products of socialization": A discussion of self-regulatory strategies and value systems. In J. Cooper \& J. M. Darley (Eds.), Attribution and social interaction: The legacy of edward e. Jones (pp. 279-292). Washington, DC: American Psychological Association.

Rohner, R. P. (1984). Toward a conception of culture for cross-cultural psychology. Journal of Cross-cultural psychology, 15, 111-138.

Rokeach, M. (1968). Beliefs, attitudes, and values. San Francisco: Jossey-Bass.

Rokeach, M. (1973). The nature of human values. New York: Free Press.

Rosch, E. (1975). Cognitive representations of semantic categories. Journal of Experimental Psychology, 104, 192-233.

Rosenberg, M. J., \& Hovland, C. I. (1960). Cognitive, affective, and behavioral components of attitude. In M. J. Rosenberg, C. I. Hovland, W. J. McGuire, R. P. Abelson \& J. W. Brehm (Eds.), Attitude organization and change: An analysis of consistency among attitude components (pp. 1-14). New Haven, CT: Yale University Press.

Rosenberg, S., \& Sedlak, A. (1972). Structural representations of implicit personality theory. In L. Berkowitz (Ed.), Advances in experimental social psychology (Vol. 6). San Diego, CA: Academic Press.

Rosenthal, D. (1955). Changes in some moral values following psychotherapy. Journal of Consulting Psychology, 19, 431-436.

Rosenthal, R. (1991). Replication in behavioral research. In J. W. Neuliep (Ed.), Replication research in the social sciences (pp. 1-30). Newbury Park, CA: Sage.

Ryan, R. M., \& Deci, E. L. (2000). Self-determination theory and the facilitation of intrinsic motivation, social development, and well-being. American Psychologist, 55, 68-78.

Ryan, R. M., \& Deci, E. L. (2002). Overview of self-determination theory: An organismic dialectical perspective. In E. L. Deci \& R. M. Ryan (Eds.), Handbook of self-determination research (pp. 3-36). Rochester, NY: University of Rochester Press.

Schimmack, U., Oishi, S., \& Diener, E. (2005). Individualism: A valid and important dimension of cultural differences between nations. Personality and Social Psychology Review, 9, 17-31. 
Schmidt, P. F. (1988). Moral values of adolescents: Public versus Christian schools. Journal of Psychology and Christianity, 7, 50-54.

Schwartz, S. (1996). Value priorities and behavior: Applying a theory of integrated value systems. In C. Seligman, J. M. Olson \& M. P. Zanna (Eds.), The psychology of values: The Ontario Symposium (Vol. 8, pp. 1-24). Hillsdale, NJ, England: Lawrence Erlbaum Associates.

Schwartz, S. H. (1992). Universals in the content and structure of values: Theoretical advances and empirical tests in 20 countries. Advances in Experimental Social Psychology, 25, 1-65.

Schwartz, S. H. (1994a). Are there universal aspects in the structure and contents of human values? Journal of Social Issues, 50, 19-45.

Schwartz, S. H. (1994b). Beyond individualism/collectivism. New cultural dimensions of values. In U. Kim, H. C. Triandis, Ç. Kağıtçıbaşı, S. Choi \& G. Yoon (Eds.), Individualism and collectivism: Theory, method, and applications (pp. 85-119). Thousand Oaks, CA, US: Sage Publications.

Schwartz, S. H. (2003). A proposal for measuring value orientations across nations [Chapter 7 in the questionnaire development report of the European social survey]. Retrieved from http://www.europeansocialsurvey.org/index.php?option=com_docman\&task=do c view\&gid=126\&itemid $=80$, January 2009.

Schwartz, S. H. (2004). Mapping and interpreting cultural differences around the world. In H. Vinken, J. Soeters \& P. Ester (Eds.), Comparing cultures, dimensions of culture in a comparative perspective (pp. 43-73). Leiden, The Netherlands: Brill.

Schwartz, S. H. (2005). Basic human values: Their content and structure across countries. In A. Tamayo \& J. B. Porto (Eds.), Valores e comportamento nas organizações [values and behavior in organizations] (pp. 21-55). Petrópolis, Brazil: Vozes.

Schwartz, S. H. (2006a). Les valeurs de base de la personne: Théorie, mesures et applications [Basic human values: Theory, measurement, and applications]. Revue Française de Sociologie, 47, 249-288.

Schwartz, S. H. (2006b). A theory of cultural value orientations: Explication and applications. Comparative Sociology, 5, 136-182.

Schwartz, S. H. (2007a). Draft users manual: Proper use of the Schwartz value survey. Retrieved from http://www.crossculturalcentre.homestead.com, September 2007.

Schwartz, S. H. (2007b). Universalism values and the inclusiveness of our moral universe. Journal of Cross-Cultural Psychology, 38, 711-728.

Schwartz, S. H. (2007c). Value orientations: Measurement, antecedents and consequences across nations. In R. Jowell, C. Roberts, R. Fitzgerald \& G. Eva (Eds.), Measuring attitudes cross-nationally - lessons from the European social survey (pp. 169-203). London: Sage Publications.

Schwartz, S. H., \& Bardi, A. (2000). Moral dialogue across cultures: An empirical perspective. In E. W. Lehmann (Ed.), Autonomy and order: An anthology of communitarian thought (pp. 155-184). Lanham, MD: Rowman \& Littlefield.

Schwartz, S. H., \& Bardi, A. (2001). Value hierarchies across cultures. Taking a similarities perspective. Journal of Cross-Cultural Psychology, 32, 268-290.

Schwartz, S. H., \& Bilsky, W. (1987). Toward a universal psychological structure of human values. Journal of Personality and Social Psychology, 53, 550-562.

Schwartz, S. H., \& Bilsky, W. (1990). Toward a theory of the universal content and structure of values: Extensions and cross-cultural replications. Journal of Personality and Social Psychology, 58, 878-891. 
Schwartz, S. H., \& Boehnke, K. (2004). Evaluating the structure of human values with confirmatory factor analysis. Journal of Research in Personality 38, 230-255.

Schwartz, S. H., Melech, G., Lehmann, A., Burgess, S., \& Harris, M. (2001). Extending the cross-cultural validity of the theory of basic human values with a different method of measurement. Journal of Cross-Cultural Psychology, 32, 519-542.

Schwartz, S. H., \& Sagiv, L. (1995). Identifying culture-specifics in the content and structure of values. Journal of Cross-Cultural Psychology, 26, 92-116.

Schwartz, S. H., \& Tessler, R. C. (1972). A test of a model for reducing measured attitude-behavior discrepancies. Journal of Personality and Social Psychology, 24, 225-236.

Scott, W. A. (1959). Empirical assessment of values and ideologies. American Sociological Review, 24, 299-309.

Seale, C. (1999). Quality in qualitative research. Qualitative Inquiry, 5, 465-478.

Segall, A. H. (1984). More than we need to know about culture, but are afraid not to ask. Journal of Cross-Cultural Psychology, 15, 153-162.

Seligman, M. E. P., Steen, T. A., Park, N., \& Peterson, C. (2005). Positive psychology progress. Empirical validation of interventions. American Psychologist, 60, 410421.

Shen, J., \& Yuan, B.-J. (1999). Moral values of only and sibling children in mainland china. The Journal of Psychology, 133, 115-124.

Shweder, R. A. (2003). Why do men barbecue?: Recipes for cultural psychology. Cambridge, MA: Harvard University Press.

Shweder, R. A., \& Haidt, J. (1993). The future of moral psychology: Truth, intuition, and the pluralist way. Psychological Science, 4, 360-365.

Shweder, R. A., \& Haidt, J. (2000). The cultural psychology of the emotions: Ancient and new. In M. Lewis \& J. M. Haviland-Jones (Eds.), Handbook of emotions (2nd ed., pp. 397-414). New York: Guildford Press.

Shweder, R. A., Mahapatra, M., \& Miller, J. (1987). Culture and moral development. In J. Kagan \& S. Lamb (Eds.), The emergence of morality in young children (pp. 183). Chicago: University of Chicago Press.

Shweder, R. A., Much, N. C., Mahapatra, M., \& Park, L. (1997). The "Big three" Of morality (autonomy, community, and divinity), and the "Big three" Explanations of suffering. In A. Brandt \& P. Rozin (Eds.), Morality and health (pp. 119-169). New York: Routledge.

Sikula, A., Sr., \& Costa, A. D. (1994). Are age and ethics related? The Journal of Psychology, 128, 659-665.

Singh, B., \& Forsyth, D. R. (1989). Sexual attitudes and moral values: The importance of idealism and relativism. Bulletin of the Psychonomic Society, 27, 160-162.

Skitka, L. J. (2002). Do the means always justify the ends, or do the ends sometimes justify the means? A value protection model of justice reasoning. Personality and Social Psychology Bulletin, 28, 588-597.

Skitka, L. J., \& Bauman, C. W. (2008). Moral conviction and political engagement. Political Psychology, 29, 29-54.

Skitka, L. J., Bauman, C. W., \& Sargis, E. G. (2005). Moral conviction: Another contributor to attitude strength or something more? Journal of Personality and Social Psychology, 88, 895-917.

Skitka, L. J., \& Houston, D. A. (2001). When due process is of no consequence: Moral mandates and presumed defendant guilt or innocence. Social justice research, $14,305-325$.

Skitka, L. J., \& Mullen, E. (2002). Understanding judgments of fairness in a real-world political context: A test of the value protection model of justice reasoning. Personality and Social Psychology Bulletin, 28, 1419-1429. 
Smetana, J. G. (1981). Preschool children's conceptions of moral and social rules. Child Development, 52, 1333-1336.

Smetana, J. G. (1982). Concepts of self and morality: Women's reasoning about abortion. New York: Praeger.

Smith, K. D., Türk Smith, S., \& Christopher, J. C. (2007). What defines the good person? Cross-cultural comparisons of experts' models with lay prototypes. Journal of Cross-Cultural Psychology, 38, 333-360.

Smith, M. B. (1969). Personal values in the study of lives. In M. B. Smith (Ed.), Social psychology and human values (pp. 97-116). Chicago: Aldine.

Smith, P. B. (2002). Levels of analysis in cross-cultural psychology. Online Readings in Psychology and Culture, Unit 2, Chapter 7. Retrieved from http://www.wwu.edu/ culture, June 2009.

Smith, P. B. (2004). Acquiescent response bias as an aspect of cultural communication style. Journal of Cross-Cultural Psychology, 35, 50-61.

Smith, P. B., Bond, M. H., \& Kağıtçıbaşı, Ç. (2006). Understanding Social Psychology across cultures: Living and working in a changing world. Thousand Oaks, CA: Sage.

Smith, P. B., \& Fischer, R. (2008). Acquiescence, extreme response bias and levels of cross-cultural analysis. In F. J. R. van de Vijver, D. A. van Hemert \& Y. H. Poortinga (Eds.), Individuals and cultures in multi-level analysis (pp. 283-311). Mahwah, NJ: Lawrence Erlbaum Associates.

Smith, P. B., Peterson, M. F., \& Schwartz, S. H. (2002). Cultural values, sources of guidance, and their relevance to managerial behavior: A 47-nation study. Journal of Cross-Cultural Psychology, 33, 188-208.

Smith, P. B., \& Schwartz, S. H. (1997). Values. In J. W. Berry, M. H. Segall \& Ç. Kağıtçıbaşı (Eds.), Handbook of cross-cultural psychology (2nd ed., Vol. 3, pp. 77-118). Needham Heights, MA: Allyn \& Bacon.

Sobel, M. E. (1982). Asymptotic confidence intervals for indirect effects in structural equation models. Sociological Methodology, 13, 290-312.

Spini, D. (2003). Measurement equivalence of 10 value types from the schwartz value survey across 21 countries. Journal of Cross-Cultural Psychology, 34, 3-23.

Stein, R., \& Nemeroff, C. J. (1995). Moral overtones of food: Judgments of others based on what they eat. Personality and Social Psychology Bulletin, 21, 480490.

Sunar, D. (2002). The psychology of morality. Online Readings in Psychology and Culture, Unit 2, Chapter 11. Retrieved from http://www.ac.wwu.edu/ culture/index-cc.htm, Mai 2006.

Tabachnick, B. G., \& Fidell, L. S. (2007). Using multivariate statistics (5th ed.). Boston: Allyn and Bacon.

Tangney, J. P. (2002). Self-conscious emotions: The self as a moral guide. In A. Tesser, D. A. Stapel \& J. V. Wood (Eds.), Self and motivation: Emerging psychological perspectives (pp. 97-117). Washington, DC, US: American Psychological Association.

Tappan, M. B. (2006). Mediated moralities: Sociocultural approaches to moral development. In M. Killen \& J. G. Smetana (Eds.), Handbook of moral development (pp. 351-374). Mahwah, NJ, US: Lawrence Erlbaum Associates Publishers.

Thomas, A. (Ed.). (1996). Psychologie interkulturellen Handelns [Psychology of intercultural actions]. Göttingen: Hogrefe.

Tomeh, A. K. (1968). Moral values in a cross-cultural perspective. Journal of Social Psychology, 74, 137-138.

Toomela, A. (2003). How should culture be studied? Culture \& Psychology, 9, 35-45. 
Triandis, H. C. (1972). The analysis of subjective culture. New York: Wiley.

Triandis, H. C. (1989). The self and social behavior in differing cultural contexts. Psychological Review 96, 506-520.

Triandis, H. C. (1994). Culture and social behavior. New York: McGraw-Hill.

Triandis, H. C. (1995). Individualism and collectivism. Boulder: Westview Press.

Triandis, H. C. (2001). Individualism and collectivism: Past, present, and future. In D. Matsumoto (Ed.), Handbook of culture and psychology (pp. 35-50). New York: Oxford University Press.

Trivers, R. L. (1971). The evolution of reciprocal altruism. Quarterly Review of Biology, 46, 35-57.

Turiel, E. (1983). The development of social knowledge: Morality and convention. Cambridge: Cambridge University Press.

Turiel, E. (2002). The culture of morality. Cambridge: Cambridge University Press.

Turiel, E. (2008). Foreword. Journal of Moral Education, 37, 279 - 288.

Uleman, J. S., Saribay, S. A., \& Gonzalez, C. M. (2008). Spontaneous inferences, implicit impressions, and implicit theories. Annual Review of Psychology, 59, 329-360.

United Nations Development Programme (2006). Summary. Human development report. Beyond scarcity: Power, poverty and the global water crisis. New York: United Nations Development Programme.

van de Vijver, F. J. R., \& Fischer, R. (2009). Improving methodological robustness in cross-cultural organizational research. In R. S. Bhagat \& R. M. Steers (Eds.), Handbook of culture, organization, and work (pp. 491-517). Cambridge: Cambridge University Press.

van de Vijver, F. J. R., \& Leung, K. (1997). Methods and data analysis for crosscultural research. Newbury Park, CA: Sage.

van Hemert, D. A. (2003). Cross-cultural meta-analyses. Online Readings in Psychology and Culture Unit 2, Chapter 13. Retrieved from http://www.ac.wwu.edu/ culture/vanhemert.htm, March 2006.

van Hemert, D. A., Poortinga, Y. H., \& van de Vijver, F. J. R. (2007). Emotion and culture: A meta-analysis. Cognition \& Emotion, 21, 913 - 943.

van Hemert, D. A., van de Vijver, F. J. R., \& Poortinga, Y. H. (2002). The Beck Depression Inventory as a measure of subjective well-being: A cross-national study. Journal of Happiness Studies, 3, 257-286.

van Hemert, D. A., van de Vijver, F. J. R., Poortinga, Y. H., \& Georgas, J. (2002). Structural and functional equivalence of the Eysenck personality questionnaire within and between countries. Personality and Individual Differences, 33, 12291249.

Vauclair, C.-M., \& Fischer, R. (2009). Do cultural values explain individuals' moral attitudes? A cross-cultural multi-level approach. Manuscript under review.

Vauclair, C.-M., Hanke, K., Fischer, R., \& Fontaine, J. R. J. (in press). The structure of human values at the culture level: A meta-analytical replication of Schwartz's value orientations using the Rokeach value survey. Journal of Cross-Cultural Psychology.

Veenhoven, R. (1993). Happiness in nations. Rotterdam, The Netherlands: Risbo.

Vélez García, A. E., \& Ostrosky-Solís, F. (2006). From morality to moral emotions. International Journal of Psychology, 41, 348-354.

Verkasalo, M., Helkama, K., Pohjanheimo, E., Andreyeva, G. M., Dubovskaya, Y. M., Tikhomandritskaya, O. A., et al. (2008). Protestant ethic value in the Schwartz value model in five countries.Unpublished manuscript, University of Helsinki, Helsinki. 
Verkasalo, M., Lönnqvist, J.-E., Lipsanen, J., \& Helkama, K. (2009). European norms and equations for a two dimensional presentation of values as measured with Schwartz's 21-item portrait values questionnaire. European Journal of Social Psychology, 39, 780-792.

Vine, I. (1983). The nature of moral commitments. In H. Weinrich-Haste \& D. Locke (Eds.), Morality in the making (pp. 17-46). Chichester: Wiley.

Walker, L. (1991). Sex differences in moral reasoning. In W. M. Kurtines \& J. L. Gewirtz (Eds.), Handbook of moral behavior and development: Vol. 2. Research (pp. 333-364). Hillsdale, NJ: Erlbaum.

Walker, L. J., \& Frimer, J. A. (2007). Moral personality of brave and caring exemplars. Journal of Personality and Social Psychology, 93, 845-860.

Walker, L. J., \& Hennig, K. H. (2004). Differing conceptions of moral exemplarity: Just, brave, and caring. Journal of Personality and Social Psychology, 86, 629647.

Walker, L. J., \& Pitts, R. C. (1998a). Data can inform the theoretical skew in moral psychology: A rejoinder to Hart. Developmental Psychology, 34, 424425.

Walker, L. J., \& Pitts, R. C. (1998b). Naturalistic conceptions of moral maturity. Developmental Psychology, 34, 403-419.

Wan, C., Chiu, C., Peng, S., \& Tam, K. (2007). Measuring cultures through intersubjective cultural norms. Implications for predicting relative identification with two or more cultures. Journal of Cross-Cultural Psychology, 38, 213-226.

Wan, C., Chiu, C., Tam, K., Lee, S., Lau, Y. I., \& Peng, S. (2007). Perceived cultural importance and actual self-importance of values in cultural identification. Journal of Personality and Social Psychology, 92, 337-354.

Waterman, A. S. (1981). Individualism and interdependence. American Psychologist, $36,762-773$

Wierzbicka, A. (2001). What did Jesus mean? Explaining the sermon on the mount and the parables in simple and universal human concepts. New York: Oxford University Press.

Wierzbicka, A. (2006). English: Meaning and culture. New York: Oxford University Press.

Wierzbicka, A. (2007). 'moral sense'. Journal of Social, Evolutionary, and Cultural Psychology, 1, 66-85.

Wilson, J. Q. (1993). The moral sense. New York: Free Press.

Wojciszke, B. (1997). Parallels between competence- versus morality-related traits and individualistic versus collectivistic values. European Journal of Social Psychology, 27, 245-256.

Wright, J. C., Cullum, J., \& Schwab, N. (2008). The cognitive and affective dimensions of moral conviction: Implications for attitudinal and behavioral measures of interpersonal tolerance. Personality and Social Psychology Bulletin, 34, 14611476.

Wright, R. (1996). The moral animal: Evolutionary psychology and everyday life. London: Abacus.

Zavalloni, M. (1980). Values. In H. Triandis \& R. Brislin (Eds.), Handbook of Crosscultural psychology (Vol. 5, pp. 73-120.). Boston: Allyn and Bacon. 
APPENDIX A: List of references included in the meta-analysis

Addleman, J. A. (1988). Differences between students' values at two schools. Psychological Reports, 63(1), 335-338

Akiba, D., \& Klug, W. (1999). The different and the same: Reexamining East and West in a cross-cultural analysis of values. Social Behavior and Personality, 27(5), 467-473.

Al-Batsh, M. W., \& 'Abd-al-Rahman, H. (1990). The value structure of Jordan university students. Dirasat, 17(3-A), 92-136.

Allen, M. W., Ng, S. H., Ikeda, K., Jawan, J. A., Sufi, A. H., Wilson, M., et al. (2007). Two decades of change in cultural values and economic development in eight East Asian and Pacific Island nations. Journal of Cross-Cultural Psychology, 38(3), 247-269.

Andrews, J. (1973). The relationship of values to identity achievement status. Journal of Youth and Adolescence, 2(2), 133-138.

Balazs, A. L. (1990). Value congruency: The case of the "socially responsible" firm. Journal of Business Research. Special Issue: Social values, 20(2), 171-181.

Balkin, J., Brandt, D., Katz, C., \& Levin, J. (1977). Values of open admissions students, police students, and their teachers. Research in Higher Education, 7(1), 1-11.

Barátová, D., \& Turcek, K. (1999). An insight into the formation of the value system of adolescents/Pohl'ad na tvorbu hodnotového systému adolescentov. Psychológia $a$ Patopsychológia Dietata, 34(1), 35-43.

Barclay, L. K., \& Sharp, A. W. (1982). Is there really a generation gap? Value similarities and differences between a group of junior high girls and their mothers. Journal of Early Adolescence, 2(2), 163171.

Becker, B. W., \& Connor, P. E. (1981). Personal values of the heavy user of mass media. ournal of Advertising Research, 21(5), 37-43.

Becker, B. W., \& Connor, P. E. (2005). Self-selection or socialization of public- and private-sector managers? A cross-cultural values analysis. Journal of Business Research, 58(1), 111-113.

Bennett, R. R., \& Greenstein, T. (1975). The police personality: A test of the predispositional model. Journal of Police Science and Administration, 3(4), 439-445.

Bhushan, A. (1979). Values across sex and family-vocations. Journal of Psychological Researches, 23(1), 11-16.

Bidzinski, K. (1995). The value system of physically impaired and nonimpaired youth/System wartosci mlodziezy z dysfunkcja narzadu ruchu i mlodziezy sprawnej ruchowo. Psychologia Wychowawcza, 38(5), 430-439.

Bilsky, W., \& Schwartz, S. H. (1994). Values and personality. European Journal of Personality, 8(3), 163-181.

Bochenska, D. (1995). A case of international perception: Selected data from a polish-american study. Polish Psychological Bulletin, 26(2), 129-144.

Brzozowski, P. (1986). Polish version of Rokeach's value survey and its theoretical basis/Polska wersja testu wartosci rokeacha i jej teoretyczne podstawy. Przeglad Psychologiczny, 29(2), 527-540.

Brzozowski, P. (1988). Transmission of values within the family/Przekazywanie wartosci w rodzinie. Psychologia Wychowawcza, 31(2), 190-200.

Chen, S., Huang, Y., \& Zhao, G. (2001). A study on values of middle school students of Hangzhou city. Chinese Journal of Clinical Psychology, 9(3), 200-202.

Chernoff, R. A., \& Davison, G. C. (1999). Values and their relationship to HIV/AIDS risk behavior among late-adolescent and young adult college students. Cognitive Therapy and Research, 23(5), 453-468.

Chusmir, L. H., \& Parker, B. (1991). Gender and situational differences in managers' values: A look at work and home lives. Journal of Business Research, 23(4), 325-335.

Çileli, M. (2000). Change in value orientations of Turkish youth from 1989 to 1995. Journal of Psychology: Interdisciplinary and Applied, 134(3), 297-305.

Çileli, M., \& Tezer, E. (1998). Life and value orientations of Turkish university students. Adolescence, 33(129), 219-228.

Cochrane, R. (1971). Research and methodology: The structure of value systems in male and female 
prisoners. British Journal of Criminology, 11(1), 73-87.

Cochrane, R., Billig, M., \& Hogg, M. (1979). Politics and values in Britain: A test of Rokeach's twovalue model. British Journal of Social \& Clinical Psychology, 18(2), 159-167.

Connor, P. E., \& Becker, B. W. (2003). Personal value systems and decision-making styles of public managers. Public Personnel Management, 32(1), 155-180.

Cross, H. J., Doost, M., R., \& Tracy, J. J. (1970). A study of values among hippies. Proceedings of the Annual Convention of the American Psychological Association, 5(Pt. 1), 449-450.

Czerniawska, M. (2002). The empathy and value system/Empatia a system wartosci. Przeglad Psychologiczny, 45(1), 7-18.

D'Amorim, M. A. (1989-1990). Values and attitudes in relation to the future in Canadian university students/Valores e atitudes em relação ao futuro em estudantes universitários canadenses. Arquivos Brasileiros de Psicologia, 42(1), 115-126.

Darden, D. K. (1983). Values and policy: The case of legal services. Basic and Applied Social Psychology, 4(1), 29-37.

DeVito, A. J., Carlson, J. F., \& Kraus, J. (1984). Values in relation to career orientation, gender, and each other. Counseling and Values, 28(4), 202-206.

Domino, G., \& Acosta, A. (1987). The relation of acculturation and values in mexican americans. Hispanic Journal of Behavioral Sciences. Special Issue: Acculturation Research, 9 (2), 131-150.

Elsayed-Elkhouly, S. M., \& Buda, R. (1997). A cross-cultural comparison of value systems of Egyptians, Americans, Africans and Arab executives. International Journal of Commerce and Management, 7(3-4), 102-119.

Feather, N. T. (1971). Value differences in relation to ethnocentrism, intolerance of ambiguity, and dogmatism. Personality and Individual Differences, 2(4), 349-366.

Feather, N. T. (1976). Value systems of self and of Australian expatriates as perceived by indigenous students in Papua New Guinea. International Journal of Psychology, 11(2), 101-110.

Feather, N. T. (1979). Accuracy of judgment of value systems: A field study of own and attributed value priorities in Papua New Guinea. International Journal of Psychology, 14(3), 151-162.

Feather, N. T., 32(1), . . (1980). Similarity of values systems within the same nation: Evidence from Australia and Papua New Guinea. Australian Journal of Psychology, 32(1), 17-30.

Feather, N. T. (1982). Reasons for entering medical school in relation to value priorities and sex of student. Journal of Occupational Psychology, 55(2), 119-128.

Feather, N. T. (1984). Masculinity, femininity, psychological androgyny, and the structure of values. Journal of Personality and Social Psychology, 47(3), 604-620.

Feather, N. T. (1984). Protestant ethic, conservatism, and values. Journal of Personality and Social Psychology, 46(5), 1132-1141.

Feather, N. T. (1985). Attitudes, values, and attributions: Explanations of unemployment. Journal of Personality and Social Psychology, 48(4), 876-889.

Feather, N. T. (1986). Value systems across cultures: Australia and China. International Journal of Psychology, 21(6), 697-715.

Flores, P. J. (1985-1986). Alcoholism treatment and the relationship of native American cultural values to recovery. International Journal of the Addictions, 20(11-12), 1707-1726.

Freitas, K. B., \& Dixon, P. W. (1997). A cosmology of Hawaiian and Western values. Social Behavior and Personality, 25(1), 59-76.

Furnham, A. (1983). The protestant work ethic, human values and attitudes towards taxation. Journal of Economic Psychology, 3(2), 113-128.

Furnham, A. (1988). Values and vocational choice: A study of value differences in medical, nursing and psychology students. Social science \& medicine, 26(6), 613-618.

Furnham, A. F. (1984). Personality and values. Personality and Individual Differences, 5(4), 483-485.

Furnham, A. F. (1984). Value systems and anomie in three cultures. International Journal of Psychology, 19(6), 565-579.

García-Alandete, J., \& Pérez Delgado, E. (2005). Religious attitudes and values preferences in a sample of young spanish university students/Actitudes religiosas y valores en un grupo de jóvenes universitarios españoles. Anales de Psicología, 21(1), 149-169.

Gombo, R., \& Schwartz, S. H. (1989). The value system of ultra-orthodox Israeli girls in comparative perspective. Megamot, 32(3), 332-360.

Greenstein, T. (1976). Behavior change through value self-confrontation: A field experiment. Journal of Personality and Social Psychology, 34(2), 254-262.

Günther, H. (1981). An attempt to translate and adapt the Rokeach scale of values for use in Brazil/Uma tentativa de traduzir e adaptar a escala de valores de Rokeach para uso no Brasil. Arquivos Brasileiros de Psicologia, 33(3), 58-72.

Helkama, K., Uutela, A., Pohjanheimo, E., Salminen, S., Koponen, A., \& Rantanen-Väntsi, L. (2003). Moral reasoning and values in medical school: A longitudinal study in Finland. Scandinavian 
Journal of Educational Research, 47(4), 399-411.

Hodgson, A., Bohning, G., McGee, C., \& Young, B. (1997). Priorities for values of adolescents in multicultural schools. Psychological Reports, 81(3, Pt 2), 1203-1209.

Howard, A., Shudo, K., \& Umeshima, M. (1983). Motivation and values among Japanese and American managers. Personnel Psychology, 36(4), 883-898.

Huang, X., Zhang, J., \& Zhang, S. (1989). Investigation on the values of five cities' Chinese adolescent students. Acta Psychologica Sinica, 21(3), 274-283.

İmamoğlu, E. O., \& Karakitapoğlu Aygün, Z. (1999). Value preferences from 1970s to 1990s: Cohort, generation and gender differences at a Turkish university/1970'lerden 1990'lara degerler: Üniversite düzeyinde gözlenen zaman, kusak ve cinsiyet farkliliklari. Türk Psikoloji Dergisi, 14(44), 1-22.

Kamakura, W. A., \& Mazzon, J. A. (1991). Value segmentation: A model for the measurement of values and value systems. Journal of Consumer Research, 18(2), 208-218.

Katz, B., Juni, S., \& Matz, P. (2002). The values of psychoanalytic psychotherapists at two points in time (1979 vs. 1993): A cross-over comparative study. Current Psychology: Developmental, Learning, Personality, Social, 21(4), 339-361.

Katz, B., Juni, S., Shope, C., \& Tang, L. (1993). The values of Chinese students: At home and abroad. International Journal of Psychology, 28(6), 761-773.

Kauffmann, D., \& Zook, A. (1982). Reply to mellor and andre's "religious group value patterns and motive orientations": Data and comment. Journal of Psychology \& Theology, 10(3), 256-258.

Khan, J. A., \& Cross, D. G. (1984). Mental health professionals: How different are their values? American Mental Health Counselors Association Journal, 6(1), 42-51.

Krampen, G. (1979). Comparison of values of psychotherapists, alcoholics, and nonclients. Zeitschrift für Klinische Psychologie und Psychotherapie, 27(2), 146-150.

Lau, S. (1988). The value orientations of Chinese university students in Hong Kong. International Journal of Psychology, 23(5), 583-596.

Lau, S. (1992). Collectivism's individualism: Value preference, personal control, and the desire for freedom among Chinese in Mainland China, Hong Kong, and Singapore. Personality and Individual Differences, 13(3), 361-366.

Lau, S., \& Wong, A. K. (1992). Value and sex-role orientation of Chinese adolescents. International Journal of Psychology, 27(1), 3-17.

Lemieux, A., \& Sauvé, G. (1999). Values of senior citizens enrolled in a university curricula versus those who are not. Educational Gerontology, 25(1), 67-88.

Linder, F., \& Bauer, D. (1983). Perception of values among male and female undergraduate students. Perceptual and Motor Skills, 56(1), 59-63.

Lorenzo Leal, J. J., \& Hernández Ruiz, B. (1986). Analysis of human values and their organization using Rokeach's scale/Análisis de los valores humanos y su organización según la escala de Rokeach. Revista de Psicología General y Aplicada, 41 (3), 539-553.

Madden, J. M. (1976). Comparisons of judgments using rank ordering and regression models. Bulletin of the Psychonomic Society, 7(3), 295-298.

Manso-Pinto, J. F., \& Finger, A. P. (1989). Terminal and instrumental values for Chilean and Brazilian university students: An exploratory study/Valores terminales e instrumentales de estudiantes universitarios chilenos y brasileños: Un estudio exploratorio. Arquivos Brasileiros de Psicologia, 41(2), 29-33.

Martini, J. L., \& Brook, R. C. (1978). Value comparisons between alcoholics, nonalcoholics, and therapists. International Journal of the Addictions, 13(7), 1169-1176.

McCarrey, M. W., \& Weisbord-Hemmingsen, J. (1980). Impact of ethnicity and sex on personal values and socialization experiences of Canadian anglophones and francophones. Journal of Psychology: Interdisciplinary and Applied, 104(1), 129-138.

Mellor, S., \& André, J. (1980). Religious group value patterns and motive orientations. Journal of Psychology and Theology, 8(2), 129-139.

Minton, J., Shell, J., \& Solomon, L. Z. (2004). A comparative study of values and attitudes of inner-city and middle-class postpartum women. Psychological Reports, 95(1), 235-249.

Minton, J., Shell, J., \& Solomon, L. Z. (2005). Values of fathers for themselves and their newborns. Psychological Reports, 96(2), 323-333.

Minton, J., Shell, J., \& Steinberg, R. (1997). Values of postpartum women from the inner city: An exploratory study. Journal of Child and Family Studies, 6(1), 27-41.

Moore, M. (1975). Rating versus ranking in the Rokeach value survey: An Israeli comparison. European Journal of Social Psychology, 5(3), 405-408.

Moore, M. (1976). A cross-cultural comparison of value systems. European Journal of Social Psychology, 6(2), 249-254.

Musek, J. (1989). Sex, gender role, and values/Spol, spolne vloge in vrednote. Anthropos, 20(3-4), 124- 
137.

Penner, L. A., \& Anh, T. (1977). A comparison of American and Vietnamese value systems. Journal of Social Psychology, 101(2), 187-204.

Pitts, R. E. (1981). Value-group analysis of cultural values in heterogeneous populations. Journal of Social Psychology, 115(1), 109-124.

Punetha, D., \& Giles, H. Y., L. . (1987). Ethnicity and immigrant values: Religion and language choice. Journal of Language and Social Psychology.Special Issue: Language and ethnic identity, 6 (3-4), 229-241.

Ramos, E. (1973). Contemporary youth's values. Pakistan Journal of Psychology, 6(3-4), 37-44.

Rankin, W. L., \& Grube, J. W. (1980). A comparison of ranking and rating procedures for value system measurement European Journal of Social Psychology, 10(3), 233-246.

Rawls, J. (1973). Comparison of Wallace, Nixon, and Humphrey supporters along certain demographic, attitudinal, and value-system dimensions. Psychological Reports, 32(1), 35-39.

Raymond, B., \& King, S. (1973). Value systems of volunteer and non-volunteer subjects. Psychological Reports, 32(3, Pt. 2), 1303-1306.

Reiter, L., \& Steiner, E. (1978). Political values of psychotherapists and psychosocial counselors. Psychotherapie und medizinische Psychologie, 28(3), 99-106.

Reynolds, B. K. (1984). A cross-cultural study of values of Germans and Americans. International Journal of Intercultural Relations, 8(3), 269-278.

Rim, Y. (1970). Values, cognitive width, external-internal control and tendency to increase performance. Psychologia: An International Journal of Psychology in the Orient, 13 (4), 223-226.

Rim, Y. (1972). Values, intelligence, need for achievement and birth-order Revista de Psicología General y Aplicada, 27(114-115), 17-28.

Rim, Y. (1982). Self-monitoring, ethical position, personality, values, and cognitive performance Personality and Individual Differences, 3(2), 219-220.

Rim, Y. (1982). Values, Idealism, Relativism, personality and cognitive performance Personality and Individual Differences, 3(1), 81-83.

Rim, Y. (1984). Importance of values according to personality, intelligence and sex. Personality and Individual Differences, 5(2), 245-246.

Roco, M. (2001). Research on moral thinking and values hierarchies/Cercetari privind gândirea morala si ierarhia valorilor. Revista de Psihologie, 47(3-4), 151-163.

Rokeach, M. (1974). Change and stability in American value systems, 1968-1971. Public Opinion Quarterly, 38(2), 222-238.

Rokeach, M., Miller, M. G., \& Snyder, J. A. (1971). The value gap between police and policed. Journal of Social Issues, 27(2), 155-171.

Rosén, A., \& Nordquist, T. A. (1980). Ego developmental level and values in yogic community. Journal of Personality and Social Psychology, 39(6), 1152-1160.

Sikula, A., \& Costa, A. D. (1994). Are age and ethics related? Journal of Psychology: Interdisciplinary and Applied, 128(6), 659-665.

Sikula, A. F. (1973). The values and value systems of governmental executives. Public Personnel Management, 2(1), 16-22.

Sikula, A. F. (1973). The values and value systems of industrial personnel managers. Public Personnel Management, 2(4), 305-309.

Sikula, J. P., \& Sikula, A. F. (1975). Do black and white university interns differ in their basic values? Journal of Negro Education, 44(2), 200-207.

Standring, D. E. (1976). Values, value change and attitudes to occupations as a function of moral judgment level. Unpublished Master thesis, Victoria University of Wellington, Wellington.

Tate, E. D., \& Miller, G. R. (1971). Differences in value systems of persons with varying religious orientations. Journal for the Scientific Study of Religion, 10(4), 357-365.

Teahan, J. E., Adams, K. M., \& Podany, E. C. (1980). A comparison of the value structure of British and U.S. police. International Journal of Social Psychiatry, 26(4), 246-254.

Upadhyay, S. N., \& Mukherjee, M. (1977). Value system across income levels. Journal of Psychological Researches, 21(2), 105-114.

Wallace, D. H. (1973). Student activist and establishment orientations: Conflict of values or misperception? Psychological Reports, 32(3, Pt. 2), 1183-1188.

Wilson, J. P. (1983). Motives, values and moral judgments. Journal of Personality Assessment, 47(4), 414-426.

Wood, V. R., \& Howell, R. (1991). A note on Hispanic values and subcultural research: An alternative view. Journal of the Academy of Marketing Science, 19(1), 61-67. 
APPENDIX B: Figure illustrating the distribution of countries regarding their socioeconomic development and importance of $S F C$ values

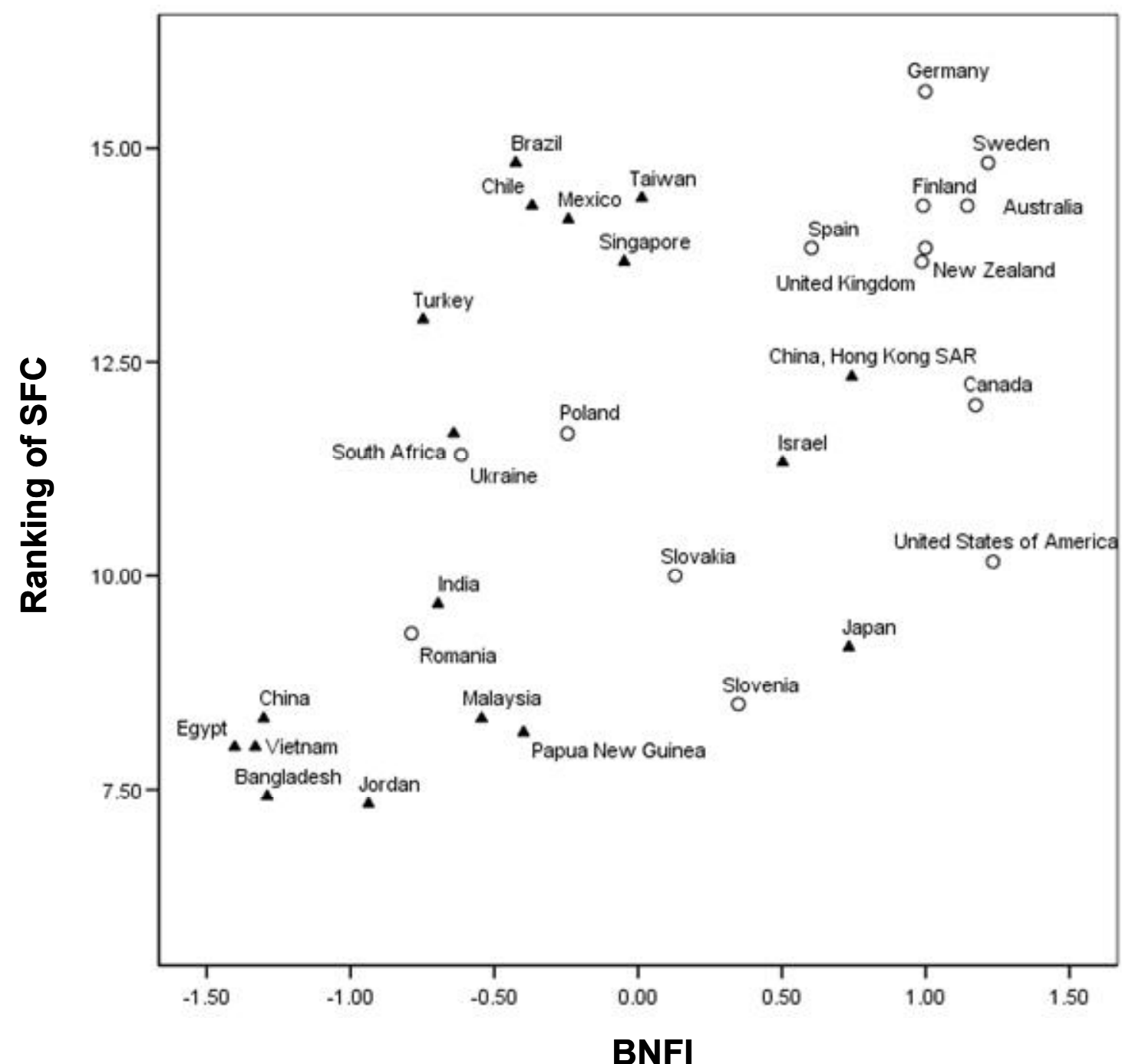

Western Countries

$\Delta$ Non-Western Countries

Note. SFC refers to Self-fulfilled Connectedness values. BNFI is a composite score consisting of: Human Development Index, GDP per capita, Gender Empowerment Measure, Political Rights, and Civil Liberties. The indices were first standardized and then averaged to combine them into the BNF Index. A higher score means higher basic need fulfillment. Higher relative ranking for SFC (Self-fulfilled Connectedness values) means lower importance of these values. 


\section{Information Sheet}

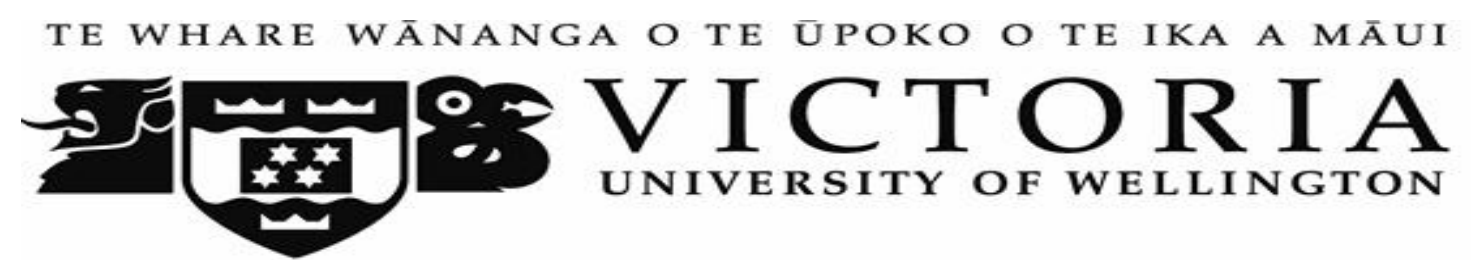

Melanie Vauclair

PhD Student

Email:

Melanie.Vauclair@vuw.ac.nz
Ronald Fischer, PhD

Senior Lecturer

Ronald.Fischer@vuw.ac.nz 463- 6548
Marc Wilson, $\mathrm{PhD}$
Senior Lecturer

Marc.Wilson@vuw.ac.nz 463-5225

\section{Dear Participant,}

The following survey is part of a cross-cultural research project on values.

\section{What is the purpose of this research?}

Cross-cultural psychologists think that values can explain much of the differences that exist between cultures. There are many different types of values and we are especially interested in personal and moral values. This research will allow us to examine which values are regarded as moral values and which ones are seen as personal values. The final interest of this research lies in the question whether people from different cultures make a similar distinction between moral and personal values.

Who is conducting the research?

- Melanie Vauclair is a PhD student. Dr Ronald Fischer and Dr Marc Wilson are supervising this project. This research has been approved by the University ethics committee.

What is involved if you agree to participate?

- If you agree to participate in this study you will be given a questionnaire that takes about 30 minutes to complete.

- During the research you are free to withdraw, without any penalty, at any point before your data have been collected.

Privacy and Confidentiality

- We will keep your data for at least five years after publication.

- You will never be identified in my research project or in any other presentation or publication. The information you provide will be coded by number only.

- In accordance with the requirements of some scientific journals and organisations, your coded data may be shared with other competent researchers.

- Your coded data may be used in other, related studies.

- A copy of the coded data will remain in the custody of Melanie Vauclair and Dr Ronald Fischer.

What happens to the information that you provide?

The data you provide may be used for one or more of the following purposes:

- The overall findings may be submitted for publication in a scientific journal, or presented at scientific conferences.

- The overall findings will form part of a PhD thesis, Masters thesis, or Honours research project that will be submitted for assessment.

If you would like to know the results of this study, they will be available approximately in December 2007 from the following sources:

- posted on the CACR webpage: http://www.vuw.ac.nz/cacr/

- Emailed to you (please write an email to Melanie.Vauclair@vuw.ac.nz to request a summary of the results).

If you have any further questions regarding this study please contact any one of us above.

\section{Thank you.}


Values play an important part in our lives. However, different people may hold different values and not every value may have the same meaning to everybody.

In the following section, you will be given 23 values that represent possible "guiding principles in someone's life". Next to each value you will find a short description in brackets that should clarify what this value means. For each of the 23 values, you will be given always the same five statements and you are asked to indicate to what extent you think this statement (e.g. ... important to me) or its opposite (e.g. ...unimportant to me) is true. Please note, that each statement or its opposite can be "extremely", "quite" or "slightly" true. If you choose the middle category it means that neither one nor the other alternative is true, OR both are equally true OR you do not know what to decide for.

If it says "in my society" it refers to the country in which you have been living for most of the time or the country you identify with.

Please write down which country this would be:

Try to answer the value section as quickly as possible.

Here is an example:

HAPPINESS (to be able to enjoy life) is ...

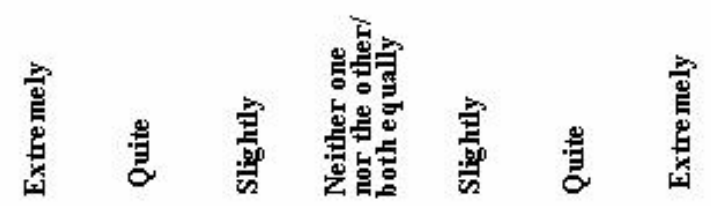

\begin{tabular}{|c|c|c|c|c|c|c|c|c|}
\hline $\begin{array}{l}\text {... important to } \\
\text { me }\end{array}$ & $x$ & 0 & 0 & 0 & 0 & 0 & 0 & $\begin{array}{l}\text {... unimportant } \\
\text { to me }\end{array}$ \\
\hline $\begin{array}{l}\text {... something } \\
\text { that I am } \\
\text { expected to } \\
\text { strive for in my } \\
\text { society }\end{array}$ & 0 & 0 & 0 & 0 & $\times$ & 0 & 0 & $\begin{array}{l}\ldots \text { something } \\
\text { that is my free } \\
\text { choice to strive } \\
\text { for in my } \\
\text { society }\end{array}$ \\
\hline $\begin{array}{l}\text {... a moral value } \\
\text { to me }\end{array}$ & 0 & 87 & 0 & 0 & 0 & 0 & 0 & $\begin{array}{l}\ldots \text { an immoral } \\
\text { value to me }\end{array}$ \\
\hline $\begin{array}{l}\text {... something } \\
\text { bad to follow }\end{array}$ & 0 & 0 & 0 & 0 & 0 & 0 & $\not$ & $\begin{array}{l}\text {... something } \\
\text { good to follow }\end{array}$ \\
\hline $\begin{array}{l}\ldots \text { the right } \\
\text { thing to strive for }\end{array}$ & 0 & 8 & 0 & 0 & 0 & 0 & 0 & $\begin{array}{l}\text {... the wrong } \\
\text { thing to strive } \\
\text { for }\end{array}$ \\
\hline
\end{tabular}


Please cross out the appropriate bubble for each value characteristic to indicate to what extent this characteristic or its opposite is true for you.

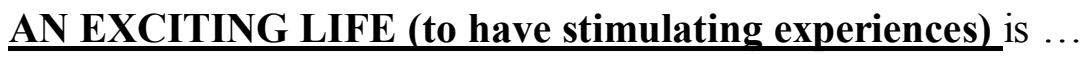

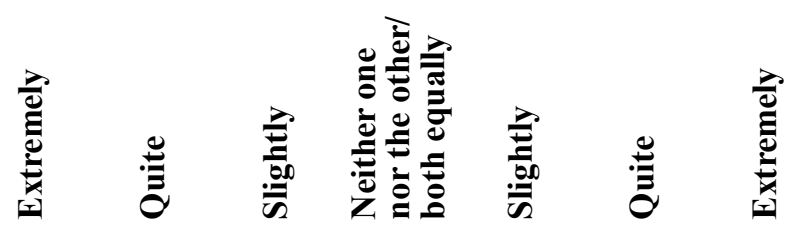

\begin{tabular}{|c|c|c|c|c|c|c|c|c|}
\hline $\begin{array}{l}\text { me important to } \\
\text { me }\end{array}$ & 0 & 0 & 0 & 0 & 0 & 0 & 0 & $\begin{array}{l}\text {... unimportant } \\
\text { to me }\end{array}$ \\
\hline $\begin{array}{l}\text {... something that } \\
\text { I am expected to } \\
\text { strive for in my } \\
\text { society }\end{array}$ & 0 & 0 & 0 & 0 & 0 & 0 & 0 & $\begin{array}{l}\text {... something } \\
\text { that is my free } \\
\text { choice to strive } \\
\text { for in my society }\end{array}$ \\
\hline $\begin{array}{l}\text {... a moral value } \\
\text { to me }\end{array}$ & 0 & 0 & 0 & 0 & 0 & 0 & 0 & \begin{tabular}{|l|}
$\ldots$ an immoral \\
value to me
\end{tabular} \\
\hline $\begin{array}{l}\ldots \text { something bad } \\
\text { to follow }\end{array}$ & 0 & 0 & 0 & 0 & 0 & 0 & 0 & $\begin{array}{l}\text {... something } \\
\text { good to follow }\end{array}$ \\
\hline $\begin{array}{l}\ldots \text { the right thing } \\
\text { to strive for }\end{array}$ & 0 & 0 & 0 & 0 & 0 & 0 & 0 & $\begin{array}{l}\text {... the wrong } \\
\text { thing to strive for }\end{array}$ \\
\hline
\end{tabular}

2. To be AMBITIOUS (to be hardworking, aspiring) is ...

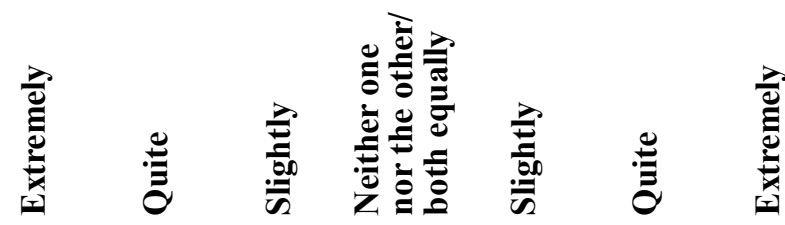

\begin{tabular}{|c|c|c|c|c|c|c|c|c|}
\hline $\begin{array}{l}\text { me important to } \\
\text { me }\end{array}$ & 0 & 0 & 0 & 0 & 0 & 0 & 0 & $\begin{array}{l}\text {... unimportant } \\
\text { to me }\end{array}$ \\
\hline $\begin{array}{l}\text {... something that } \\
\text { I am expected to } \\
\text { strive for in my } \\
\text { society }\end{array}$ & 0 & 0 & 0 & 0 & 0 & 0 & 0 & $\begin{array}{l}\text {... something } \\
\text { that is my free } \\
\text { choice to strive } \\
\text { for in my society }\end{array}$ \\
\hline $\begin{array}{l}\text {... a moral value } \\
\text { to me }\end{array}$ & 0 & 0 & 0 & 0 & 0 & 0 & 0 & \begin{tabular}{|l|}
... an immoral \\
value to me
\end{tabular} \\
\hline $\begin{array}{l}\ldots \text { something bad } \\
\text { to follow }\end{array}$ & 0 & 0 & 0 & 0 & 0 & 0 & 0 & $\begin{array}{l}\text {... something } \\
\text { good to follow }\end{array}$ \\
\hline $\begin{array}{l}\ldots \text { the right thing } \\
\text { to strive for }\end{array}$ & 0 & 0 & 0 & 0 & 0 & 0 & 0 & $\begin{array}{l}\ldots \text { the wrong } \\
\text { thing to strive for }\end{array}$ \\
\hline
\end{tabular}


3. ACCEPTING MY PORTION IN LIFE (to submit to life's circumstances) is ...

\begin{tabular}{|c|c|c|c|c|c|c|c|c|}
\hline & 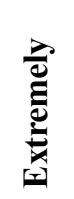 & $\stackrel{\mathscr{\Xi}}{\partial}$ & 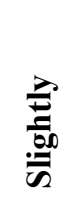 & 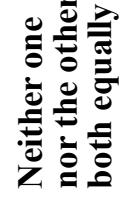 & 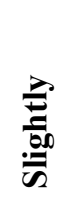 & $\stackrel{\mathscr{\Xi}}{\partial}$ & 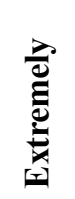 & \\
\hline me important to & 0 & 0 & 0 & 0 & 0 & 0 & 0 & $\begin{array}{l}\ldots \text { unimportant } \\
\text { to me }\end{array}$ \\
\hline $\begin{array}{l}\text {... something that } \\
\text { I am expected to } \\
\text { strive for in my } \\
\text { society }\end{array}$ & 0 & 0 & 0 & 0 & 0 & 0 & 0 & $\begin{array}{l}\text {... something } \\
\text { that is my free } \\
\text { choice to strive } \\
\text { for in my society }\end{array}$ \\
\hline $\begin{array}{l}\text {... a moral value } \\
\text { to me }\end{array}$ & 0 & 0 & 0 & 0 & 0 & 0 & 0 & $\begin{array}{l}\text {... an immoral } \\
\text { value to me }\end{array}$ \\
\hline $\begin{array}{l}\text {... something bad } \\
\text { to follow }\end{array}$ & 0 & 0 & 0 & 0 & 0 & 0 & 0 & $\begin{array}{l}\text {... something } \\
\text { good to follow }\end{array}$ \\
\hline $\begin{array}{l}\ldots \text { the right thing } \\
\text { to strive for }\end{array}$ & 0 & 0 & 0 & 0 & 0 & 0 & 0 & $\begin{array}{l}\ldots \text { the wrong } \\
\text { thing to strive for }\end{array}$ \\
\hline
\end{tabular}

4. A WORLD OF BEAUTY (to appreciate the beauty of nature and the arts) is ...

\begin{tabular}{|c|c|c|c|c|c|c|c|c|}
\hline & 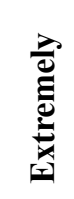 & $\stackrel{\mathscr{\Xi}}{\ddot{\sigma}}$ & 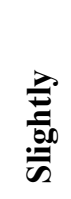 & 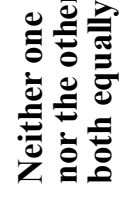 & 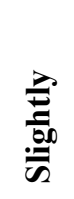 & $\stackrel{\mathscr{\Xi}}{0}$ & 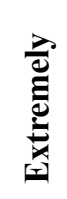 & \\
\hline me important to & 0 & 0 & 0 & 0 & 0 & 0 & 0 & $\begin{array}{l}\ldots \text { unimportant } \\
\text { to me }\end{array}$ \\
\hline $\begin{array}{l}\text {... something that } \\
\text { I am expected to } \\
\text { strive for in my } \\
\text { society }\end{array}$ & 0 & 0 & 0 & 0 & 0 & 0 & 0 & $\begin{array}{l}\text {... something } \\
\text { that is my free } \\
\text { choice to strive } \\
\text { for in my society }\end{array}$ \\
\hline $\begin{array}{l}\text {... a moral value } \\
\text { to me }\end{array}$ & 0 & 0 & 0 & 0 & 0 & 0 & 0 & $\begin{array}{l}\ldots . \text { an immoral } \\
\text { value to me }\end{array}$ \\
\hline $\begin{array}{l}\text {... something bad } \\
\text { to follow }\end{array}$ & 0 & 0 & 0 & 0 & 0 & 0 & 0 & $\begin{array}{l}\text {... something } \\
\text { good to follow }\end{array}$ \\
\hline $\begin{array}{l}\ldots \text { the right thing } \\
\text { to strive for }\end{array}$ & 0 & 0 & 0 & 0 & 0 & 0 & 0 & $\begin{array}{l}\ldots \text { the wrong } \\
\text { thing to strive for }\end{array}$ \\
\hline
\end{tabular}




\section{AUTHORITY (to take the right to lead and command) is ...}

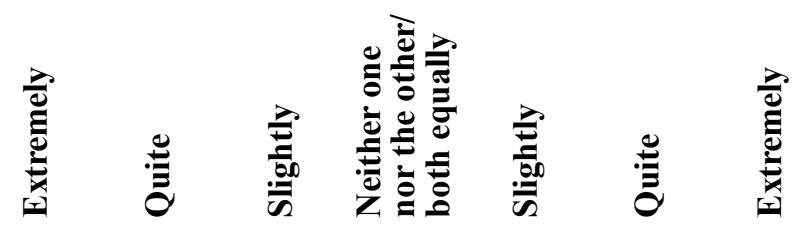

\begin{tabular}{|c|c|c|c|c|c|c|c|c|}
\hline $\begin{array}{l}\text { me important to } \\
\text { me }\end{array}$ & 0 & 0 & 0 & 0 & 0 & 0 & 0 & $\begin{array}{l}\ldots \text { unimportant } \\
\text { to me }\end{array}$ \\
\hline $\begin{array}{l}\text {... something that } \\
\text { I am expected to } \\
\text { strive for in my } \\
\text { society }\end{array}$ & 0 & 0 & 0 & 0 & 0 & 0 & 0 & $\begin{array}{l}\text {... something } \\
\text { that is my free } \\
\text { choice to strive } \\
\text { for in my society }\end{array}$ \\
\hline $\begin{array}{l}\text {... a moral value } \\
\text { to me }\end{array}$ & 0 & 0 & 0 & 0 & 0 & 0 & 0 & $\begin{array}{l}\text {... an immoral } \\
\text { value to me }\end{array}$ \\
\hline $\begin{array}{l}\text {... something bad } \\
\text { to follow }\end{array}$ & 0 & 0 & 0 & 0 & 0 & 0 & 0 & $\begin{array}{l}\ldots \text { something } \\
\text { good to follow }\end{array}$ \\
\hline $\begin{array}{l}\text {... the right thing } \\
\text { to strive for }\end{array}$ & 0 & 0 & 0 & 0 & 0 & 0 & 0 & $\begin{array}{l}\text {... the wrong } \\
\text { thing to strive for }\end{array}$ \\
\hline
\end{tabular}

6. To be BROAD-MINDED (to tolerate different ideas and beliefs) is ...

\begin{tabular}{|c|c|c|c|c|c|c|c|c|}
\hline & 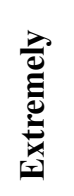 & $\stackrel{\mathscr{\Xi}}{\Xi}$ & 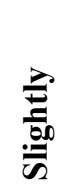 & 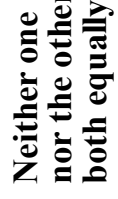 & 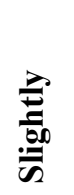 & $\stackrel{\mathscr{E}}{\partial}$ & & \\
\hline $\begin{array}{l}\text { me important to } \\
\text { me }\end{array}$ & 0 & 0 & 0 & 0 & 0 & 0 & 0 & $\begin{array}{l}\text {... unimportant } \\
\text { to me }\end{array}$ \\
\hline $\begin{array}{l}\text {... something that } \\
\text { I am expected to } \\
\text { strive for in my } \\
\text { society }\end{array}$ & 0 & 0 & 0 & 0 & 0 & 0 & 0 & $\begin{array}{l}\text {... something } \\
\text { that is my free } \\
\text { choice to strive } \\
\text { for in my society }\end{array}$ \\
\hline $\begin{array}{l}\text {... a moral value } \\
\text { to me }\end{array}$ & 0 & 0 & 0 & 0 & 0 & 0 & 0 & $\begin{array}{l}\text {... an immoral } \\
\text { value to me }\end{array}$ \\
\hline $\begin{array}{l}\text {... something bad } \\
\text { to follow }\end{array}$ & 0 & 0 & 0 & 0 & 0 & 0 & 0 & $\begin{array}{l}\text {... something } \\
\text { good to follow }\end{array}$ \\
\hline $\begin{array}{l}\text {... the right thing } \\
\text { to strive for }\end{array}$ & 0 & 0 & 0 & 0 & 0 & 0 & 0 & $\begin{array}{l}\ldots \text { the wrong } \\
\text { thing to strive for }\end{array}$ \\
\hline
\end{tabular}




\section{To CHOOSE ONE'S OWN GOALS (to select one's own purposes) is ...}

\begin{tabular}{|c|c|c|c|c|c|c|c|c|}
\hline & 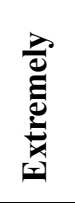 & $\stackrel{\mathscr{E}}{0}$ & 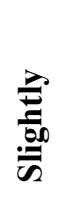 & 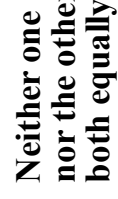 & 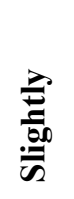 & $\stackrel{\mathscr{\Xi}}{0}$ & : & \\
\hline $\begin{array}{l}\text { me important to } \\
\text { me }\end{array}$ & 0 & 0 & 0 & 0 & 0 & 0 & 0 & $\begin{array}{l}\text {... unimportant } \\
\text { to me }\end{array}$ \\
\hline $\begin{array}{l}\text {... something that } \\
\text { I am expected to } \\
\text { strive for in my } \\
\text { society }\end{array}$ & 0 & 0 & 0 & 0 & 0 & 0 & 0 & $\begin{array}{l}\text {... something } \\
\text { that is my free } \\
\text { choice to strive } \\
\text { for in my society }\end{array}$ \\
\hline $\begin{array}{l}\ldots \text { a moral value } \\
\text { to me }\end{array}$ & 0 & 0 & 0 & 0 & 0 & 0 & 0 & $\begin{array}{l}\text {... an immoral } \\
\text { value to me }\end{array}$ \\
\hline $\begin{array}{l}\text {... something bad } \\
\text { to follow }\end{array}$ & 0 & 0 & 0 & 0 & 0 & 0 & 0 & $\begin{array}{l}\text {... something } \\
\text { good to follow }\end{array}$ \\
\hline $\begin{array}{l}\ldots \text { the right thing } \\
\text { to strive for }\end{array}$ & 0 & 0 & 0 & 0 & 0 & 0 & 0 & $\begin{array}{l}\ldots \text { the wrong } \\
\text { thing to strive for }\end{array}$ \\
\hline
\end{tabular}

8. CLEAN (to lay emphasis on neatness and tidiness) is ...

\begin{tabular}{|c|c|c|c|c|c|c|c|c|}
\hline & 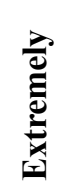 & $\stackrel{\mathscr{\Xi}}{\Xi}$ & 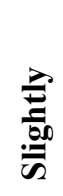 & 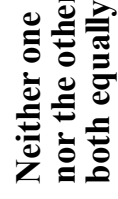 & 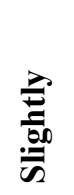 & $\stackrel{\mathscr{\Xi}}{0}$ & 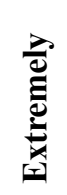 & \\
\hline $\begin{array}{l}\text {... important to } \\
\text { me }\end{array}$ & 0 & 0 & 0 & 0 & 0 & 0 & 0 & $\begin{array}{l}\text {... unimportant } \\
\text { to me }\end{array}$ \\
\hline $\begin{array}{l}\text {... something that } \\
\text { I am expected to } \\
\text { strive for in my } \\
\text { society }\end{array}$ & 0 & 0 & 0 & 0 & 0 & 0 & 0 & $\begin{array}{l}\text {... Something } \\
\text { that is my free } \\
\text { choice to strive } \\
\text { for in my society }\end{array}$ \\
\hline $\begin{array}{l}\text {... a moral value } \\
\text { to me }\end{array}$ & 0 & 0 & 0 & 0 & 0 & 0 & 0 & $\begin{array}{l}\text {... an immoral } \\
\text { value to me }\end{array}$ \\
\hline $\begin{array}{l}\text {... something bad } \\
\text { to follow }\end{array}$ & 0 & 0 & 0 & 0 & 0 & 0 & 0 & $\begin{array}{l}\text {... something } \\
\text { good to follow }\end{array}$ \\
\hline $\begin{array}{l}\text {... the right thing } \\
\text { to strive for }\end{array}$ & 0 & 0 & 0 & 0 & 0 & 0 & 0 & $\begin{array}{l}\ldots \text { the wrong } \\
\text { thing to strive for }\end{array}$ \\
\hline
\end{tabular}


9. To be DARING (to seek adventure, to take risks) is ...

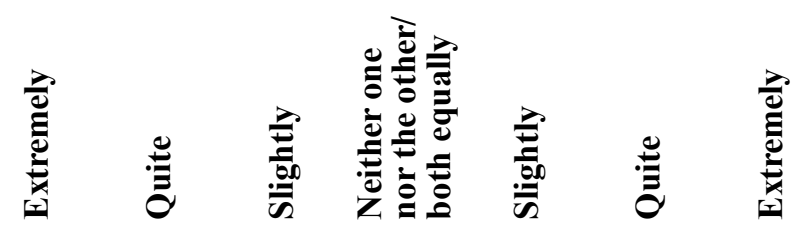

\begin{tabular}{|c|c|c|c|c|c|c|c|c|}
\hline $\begin{array}{l}\text { me important to } \\
\text { me }\end{array}$ & 0 & 0 & 0 & 0 & 0 & 0 & 0 & $\begin{array}{l}\text {... unimportant } \\
\text { to me }\end{array}$ \\
\hline $\begin{array}{l}\text {... something that } \\
\text { I am expected to } \\
\text { strive for in my } \\
\text { society }\end{array}$ & 0 & 0 & 0 & 0 & 0 & 0 & 0 & $\begin{array}{l}\text {... something } \\
\text { that is my free } \\
\text { choice to strive } \\
\text { for in my society }\end{array}$ \\
\hline $\begin{array}{l}\ldots \text { a moral value } \\
\text { to me }\end{array}$ & 0 & 0 & 0 & 0 & 0 & 0 & 0 & $\begin{array}{l}\text {... an immoral } \\
\text { value to me }\end{array}$ \\
\hline $\begin{array}{l}\ldots \text { something bad } \\
\text { to follow }\end{array}$ & 0 & 0 & 0 & 0 & 0 & 0 & 0 & $\begin{array}{l}\text {... something } \\
\text { good to follow }\end{array}$ \\
\hline $\begin{array}{l}\ldots \text { the right thing } \\
\text { to strive for }\end{array}$ & 0 & 0 & 0 & 0 & 0 & 0 & 0 & $\begin{array}{l}\text {... the wrong } \\
\text { thing to strive for }\end{array}$ \\
\hline
\end{tabular}

10. EQUALITY (to seek equal opportunity for all) is ...

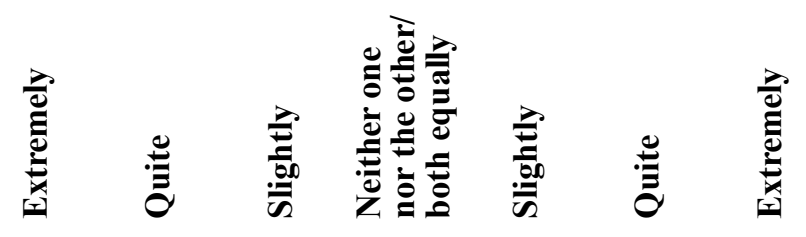

\begin{tabular}{|c|c|c|c|c|c|c|c|c|}
\hline $\begin{array}{l}\text { me important to } \\
\text { me }\end{array}$ & 0 & 0 & 0 & 0 & 0 & 0 & 0 & $\begin{array}{l}\text {... unimportant } \\
\text { to me }\end{array}$ \\
\hline $\begin{array}{l}\text {... something that } \\
\text { I am expected to } \\
\text { strive for in my } \\
\text { society }\end{array}$ & 0 & 0 & 0 & 0 & 0 & 0 & 0 & $\begin{array}{l}\text {... something } \\
\text { that is my free } \\
\text { choice to strive } \\
\text { for in my society }\end{array}$ \\
\hline $\begin{array}{l}\ldots \text { a moral value } \\
\text { to me }\end{array}$ & 0 & 0 & 0 & 0 & 0 & 0 & 0 & $\begin{array}{l}\text {... an immoral } \\
\text { value to me }\end{array}$ \\
\hline $\begin{array}{l}\ldots \text { something bad } \\
\text { to follow }\end{array}$ & 0 & 0 & 0 & 0 & 0 & 0 & 0 & $\begin{array}{l}\text {... something } \\
\text { good to follow }\end{array}$ \\
\hline $\begin{array}{l}\ldots \text { the right thing } \\
\text { to strive for }\end{array}$ & 0 & 0 & 0 & 0 & 0 & 0 & 0 & $\begin{array}{l}\text {... the wrong } \\
\text { thing to strive for }\end{array}$ \\
\hline
\end{tabular}


11. To be HELPFUL (to work for the welfare of others) is ...

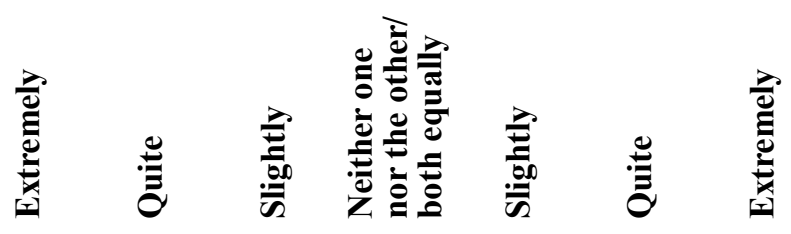

\begin{tabular}{|c|c|c|c|c|c|c|c|c|}
\hline $\begin{array}{l}\text { me important to } \\
\text { me }\end{array}$ & 0 & 0 & 0 & 0 & 0 & 0 & 0 & $\begin{array}{l}\text {... unimportant } \\
\text { to me }\end{array}$ \\
\hline $\begin{array}{l}\text {... something that } \\
\text { I am expected to } \\
\text { strive for in my } \\
\text { society }\end{array}$ & 0 & 0 & 0 & 0 & 0 & 0 & 0 & $\begin{array}{l}\text {... something } \\
\text { that is my free } \\
\text { choice to strive } \\
\text { for in my society }\end{array}$ \\
\hline $\begin{array}{l}\text {... a moral value } \\
\text { to me }\end{array}$ & 0 & 0 & 0 & 0 & 0 & 0 & 0 & $\begin{array}{l}\text {... an immoral } \\
\text { value to me }\end{array}$ \\
\hline $\begin{array}{l}\ldots \text { something bad } \\
\text { to follow }\end{array}$ & 0 & 0 & 0 & 0 & 0 & 0 & 0 & $\begin{array}{l}\text {... something } \\
\text { good to follow }\end{array}$ \\
\hline $\begin{array}{l}\ldots \text { the right thing } \\
\text { to strive for }\end{array}$ & 0 & 0 & 0 & 0 & 0 & 0 & 0 & $\begin{array}{l}\text {... the wrong } \\
\text { thing to strive for }\end{array}$ \\
\hline
\end{tabular}

12. To be HONEST (to be genuine, sincere) is ...

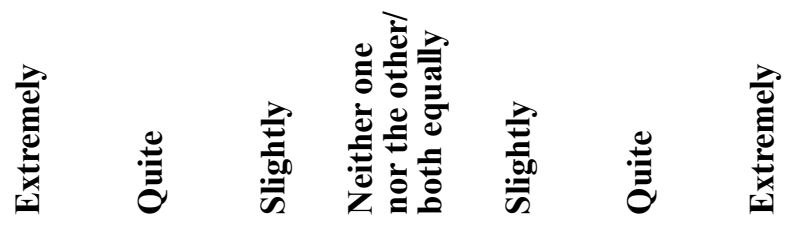

\begin{tabular}{|c|c|c|c|c|c|c|c|c|}
\hline $\begin{array}{l}\text {... important to } \\
\text { me }\end{array}$ & 0 & 0 & 0 & 0 & 0 & 0 & 0 & $\begin{array}{l}\text {... unimportant } \\
\text { to me }\end{array}$ \\
\hline $\begin{array}{l}\text {... something that } \\
\text { I am expected to } \\
\text { strive for in my } \\
\text { society }\end{array}$ & 0 & 0 & 0 & 0 & 0 & 0 & 0 & $\begin{array}{l}\text {... something } \\
\text { that is my free } \\
\text { choice to strive } \\
\text { for in my society }\end{array}$ \\
\hline $\begin{array}{l}\text {... a moral value } \\
\text { to me }\end{array}$ & 0 & 0 & 0 & 0 & 0 & 0 & 0 & $\begin{array}{l}\text {... an immoral } \\
\text { value to me }\end{array}$ \\
\hline $\begin{array}{l}\ldots \text { something bad } \\
\text { to follow }\end{array}$ & 0 & 0 & 0 & 0 & 0 & 0 & 0 & $\begin{array}{l}\text {... something } \\
\text { good to follow }\end{array}$ \\
\hline $\begin{array}{l}\ldots \text { the right thing } \\
\text { to strive for }\end{array}$ & 0 & 0 & 0 & 0 & 0 & 0 & 0 & $\begin{array}{l}\text {... the wrong } \\
\text { thing to strive for }\end{array}$ \\
\hline
\end{tabular}




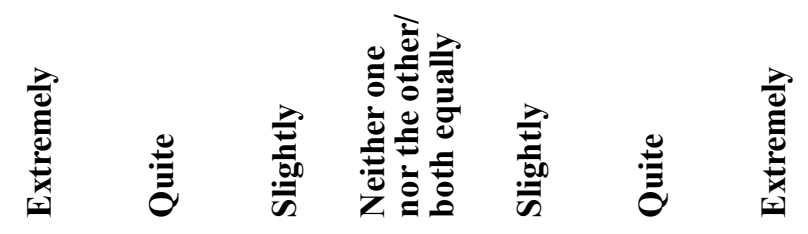

\begin{tabular}{|c|c|c|c|c|c|c|c|c|}
\hline $\begin{array}{l}\text { me important to } \\
\text { me }\end{array}$ & 0 & 0 & 0 & 0 & 0 & 0 & 0 & $\begin{array}{l}\text {... unimportant } \\
\text { to me }\end{array}$ \\
\hline $\begin{array}{l}\text {... something that } \\
\text { I am expected to } \\
\text { strive for in my } \\
\text { society }\end{array}$ & 0 & 0 & 0 & 0 & 0 & 0 & 0 & $\begin{array}{l}\text {... something } \\
\text { that is my free } \\
\text { choice to strive } \\
\text { for in my society }\end{array}$ \\
\hline $\begin{array}{l}\text {... a moral value } \\
\text { to me }\end{array}$ & 0 & 0 & 0 & 0 & 0 & 0 & 0 & $\begin{array}{l}\ldots \text { an immoral } \\
\text { value to me }\end{array}$ \\
\hline $\begin{array}{l}\ldots \text { something bad } \\
\text { to follow }\end{array}$ & 0 & 0 & 0 & 0 & 0 & 0 & 0 & $\begin{array}{l}\text {... something } \\
\text { good to follow }\end{array}$ \\
\hline $\begin{array}{l}\ldots \text { the right thing } \\
\text { to strive for }\end{array}$ & 0 & 0 & 0 & 0 & 0 & 0 & 0 & $\begin{array}{l}\text {... the wrong } \\
\text { thing to strive for }\end{array}$ \\
\hline
\end{tabular}

14. To be INDEPENDENT (to be self-reliant, self-sufficient) is ...

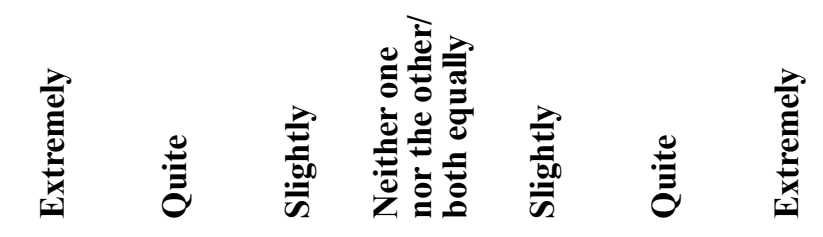

\begin{tabular}{|c|c|c|c|c|c|c|c|c|}
\hline me important to & 0 & 0 & 0 & 0 & 0 & 0 & 0 & $\begin{array}{l}\text {... unimportant } \\
\text { to me }\end{array}$ \\
\hline $\begin{array}{l}\text {... something that } \\
\text { I am expected to } \\
\text { strive for in my } \\
\text { society }\end{array}$ & 0 & 0 & 0 & 0 & 0 & 0 & 0 & $\begin{array}{l}\text {... something } \\
\text { that is my free } \\
\text { choice to strive } \\
\text { for in my society }\end{array}$ \\
\hline $\begin{array}{l}\text {... a moral value } \\
\text { to me }\end{array}$ & 0 & 0 & 0 & 0 & 0 & 0 & 0 & $\begin{array}{l}\ldots \text { an immoral } \\
\text { value to me }\end{array}$ \\
\hline $\begin{array}{l}\text {... something bad } \\
\text { to follow }\end{array}$ & 0 & 0 & 0 & 0 & 0 & 0 & 0 & $\begin{array}{l}\text {... something } \\
\text { good to follow }\end{array}$ \\
\hline $\begin{array}{l}\ldots \text { the right thing } \\
\text { to strive for }\end{array}$ & 0 & 0 & 0 & 0 & 0 & 0 & 0 & $\begin{array}{l}\ldots \text { the wrong } \\
\text { thing to strive for }\end{array}$ \\
\hline
\end{tabular}


15. INTEGRITY ${ }^{30}$ (to walk the talk; to practice what one preaches) is ...

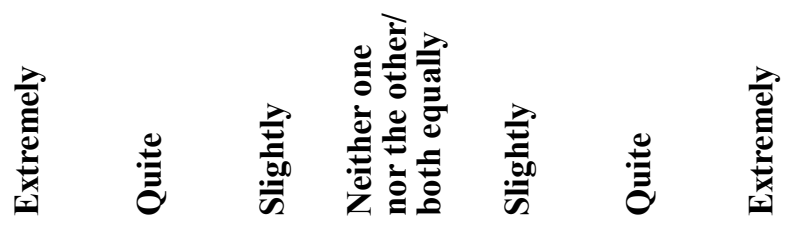

\begin{tabular}{|c|c|c|c|c|c|c|c|c|}
\hline $\begin{array}{l}\text { me important to } \\
\text { me }\end{array}$ & 0 & 0 & 0 & 0 & 0 & 0 & 0 & $\begin{array}{l}\text {... unimportant } \\
\text { to me }\end{array}$ \\
\hline $\begin{array}{l}\text {... something that } \\
\text { I am expected to } \\
\text { strive for in my } \\
\text { society }\end{array}$ & 0 & 0 & 0 & 0 & 0 & 0 & 0 & $\begin{array}{l}\text {... Something } \\
\text { that is my free } \\
\text { choice to strive } \\
\text { for in my society }\end{array}$ \\
\hline $\begin{array}{l}\text {... a moral value } \\
\text { to me }\end{array}$ & 0 & 0 & 0 & 0 & 0 & 0 & 0 & $\begin{array}{l}\text {... an immoral } \\
\text { value to me }\end{array}$ \\
\hline $\begin{array}{l}\ldots \text { something bad } \\
\text { to follow }\end{array}$ & 0 & 0 & 0 & 0 & 0 & 0 & 0 & $\begin{array}{l}\text {... something } \\
\text { good to follow }\end{array}$ \\
\hline $\begin{array}{l}\ldots \text { the right thing } \\
\text { to strive for }\end{array}$ & 0 & 0 & 0 & 0 & 0 & 0 & 0 & $\begin{array}{l}\text {... the wrong } \\
\text { thing to strive for }\end{array}$ \\
\hline
\end{tabular}

16. To be MODERATE (to avoid extremes of feeling and action) is ...

\begin{tabular}{|c|c|c|c|c|c|c|c|c|}
\hline & 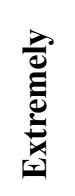 & $\stackrel{\mathscr{E}}{\mathscr{\Xi}}$ & 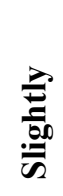 & 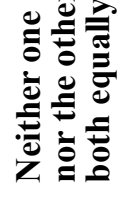 & 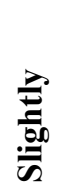 & $\stackrel{\mathscr{\Xi}}{0}$ & 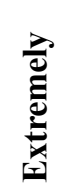 & \\
\hline $\begin{array}{l}\text { me important to } \\
\text { me }\end{array}$ & 0 & 0 & 0 & 0 & 0 & 0 & 0 & $\begin{array}{l}\text {... unimportant } \\
\text { to me }\end{array}$ \\
\hline $\begin{array}{l}\text {... something that } \\
\text { I am expected to } \\
\text { strive for in my } \\
\text { society }\end{array}$ & 0 & 0 & 0 & 0 & 0 & 0 & 0 & $\begin{array}{l}\text {... Something } \\
\text { that is my free } \\
\text { choice to strive } \\
\text { for in my society }\end{array}$ \\
\hline $\begin{array}{l}\text {... a moral value } \\
\text { to me }\end{array}$ & 0 & 0 & 0 & 0 & 0 & 0 & 0 & $\begin{array}{l}\text {... an immoral } \\
\text { value to me }\end{array}$ \\
\hline $\begin{array}{l}\ldots \text { something bad } \\
\text { to follow }\end{array}$ & 0 & 0 & 0 & 0 & 0 & 0 & 0 & $\begin{array}{l}\ldots \text { something } \\
\text { good to follow }\end{array}$ \\
\hline $\begin{array}{l}\text {... the right thing } \\
\text { to strive for }\end{array}$ & 0 & 0 & 0 & 0 & 0 & 0 & 0 & $\begin{array}{l}\ldots \text { the wrong } \\
\text { thing to strive for }\end{array}$ \\
\hline
\end{tabular}

\footnotetext{
${ }^{30}$ This value was added to the SVS in order to provide an equal number of items in the two versions of the survey. It was not included in the analysis.
} 
17. PLEASURE (to seek gratification of desires) is ...

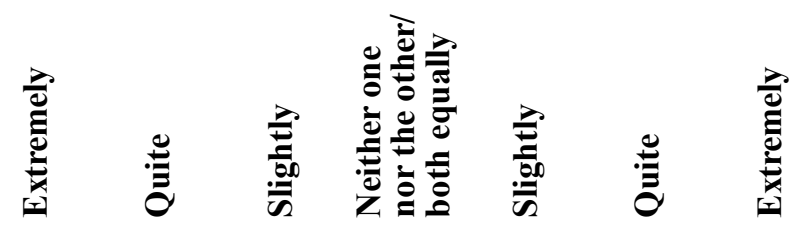

\begin{tabular}{|c|c|c|c|c|c|c|c|c|}
\hline $\begin{array}{l}\text { me important to } \\
\text { me }\end{array}$ & 0 & 0 & 0 & 0 & 0 & 0 & 0 & $\begin{array}{l}\text {... unimportant } \\
\text { to me }\end{array}$ \\
\hline $\begin{array}{l}\text {... something that } \\
\text { I am expected to } \\
\text { strive for in my } \\
\text { society }\end{array}$ & 0 & 0 & 0 & 0 & 0 & 0 & 0 & $\begin{array}{l}\text {... something } \\
\text { that is my free } \\
\text { choice to strive } \\
\text { for in my society }\end{array}$ \\
\hline $\begin{array}{l}\ldots \text { a moral value } \\
\text { to me }\end{array}$ & 0 & 0 & 0 & 0 & 0 & 0 & 0 & $\begin{array}{l}\text {... an immoral } \\
\text { value to me }\end{array}$ \\
\hline $\begin{array}{l}\ldots \text { something bad } \\
\text { to follow }\end{array}$ & 0 & 0 & 0 & 0 & 0 & 0 & 0 & $\begin{array}{l}\text {... something } \\
\text { good to follow }\end{array}$ \\
\hline $\begin{array}{l}\ldots \text { the right thing } \\
\text { to strive for }\end{array}$ & 0 & 0 & 0 & 0 & 0 & 0 & 0 & $\begin{array}{l}\text {... the wrong } \\
\text { thing to strive for }\end{array}$ \\
\hline
\end{tabular}

18. RECIPROCATION OF FAVOURS (to avoid indebtedness) is ...

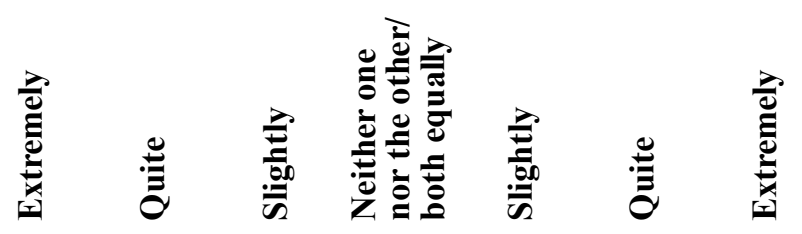

\begin{tabular}{|c|c|c|c|c|c|c|c|c|}
\hline $\begin{array}{l}\text { me important to } \\
\text { me }\end{array}$ & 0 & 0 & 0 & 0 & 0 & 0 & 0 & $\begin{array}{l}\text {... unimportant } \\
\text { to me }\end{array}$ \\
\hline $\begin{array}{l}\text {... something that } \\
\text { I am expected to } \\
\text { strive for in my } \\
\text { society }\end{array}$ & 0 & 0 & 0 & 0 & 0 & 0 & 0 & $\begin{array}{l}\text {... something } \\
\text { that is my free } \\
\text { choice to strive } \\
\text { for in my society }\end{array}$ \\
\hline $\begin{array}{l}\text {... a moral value } \\
\text { to me }\end{array}$ & 0 & 0 & 0 & 0 & 0 & 0 & 0 & $\begin{array}{l}\text {... an immoral } \\
\text { value to me }\end{array}$ \\
\hline $\begin{array}{l}\ldots \text { something bad } \\
\text { to follow }\end{array}$ & 0 & 0 & 0 & 0 & 0 & 0 & 0 & $\begin{array}{l}\text {... something } \\
\text { good to follow }\end{array}$ \\
\hline $\begin{array}{l}\ldots \text { the right thing } \\
\text { to strive for }\end{array}$ & 0 & 0 & 0 & 0 & 0 & 0 & 0 & $\begin{array}{l}\text {... the wrong } \\
\text { thing to strive for }\end{array}$ \\
\hline
\end{tabular}


19. RESPECT FOR TRADITION (to preserve time-honored customs) is...

\begin{tabular}{|c|c|c|c|c|c|c|c|c|}
\hline & 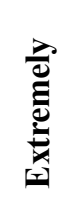 & $\stackrel{\mathscr{\Xi}}{\mathscr{\Xi}}$ & 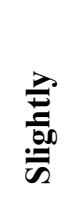 & 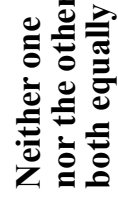 & $\begin{array}{l}\frac{2}{E} \\
\frac{.00}{\bar{b}} \\
\bar{n}\end{array}$ & $\stackrel{\mathscr{\Xi}}{0}$ & 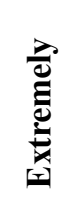 & \\
\hline me important to & 0 & 0 & 0 & 0 & 0 & 0 & 0 & $\begin{array}{l}\ldots \text { unimportant } \\
\text { to me }\end{array}$ \\
\hline $\begin{array}{l}\text {... something that } \\
\text { I am expected to } \\
\text { strive for in my } \\
\text { society }\end{array}$ & 0 & 0 & 0 & 0 & 0 & 0 & 0 & $\begin{array}{l}\text {... Something } \\
\text { that is my free } \\
\text { choice to strive } \\
\text { for in my society }\end{array}$ \\
\hline $\begin{array}{l}\text {... a moral value } \\
\text { to me }\end{array}$ & 0 & 0 & 0 & 0 & 0 & 0 & 0 & $\begin{array}{l}\text {... an immoral } \\
\text { value to me }\end{array}$ \\
\hline $\begin{array}{l}\text {... something bad } \\
\text { to follow }\end{array}$ & 0 & 0 & 0 & 0 & 0 & 0 & 0 & $\begin{array}{l}\text {... something } \\
\text { good to follow }\end{array}$ \\
\hline $\begin{array}{l}\ldots \text { the right thing } \\
\text { to strive for }\end{array}$ & 0 & 0 & 0 & 0 & 0 & 0 & 0 & $\begin{array}{l}\ldots \text { the wrong } \\
\text { thing to strive for }\end{array}$ \\
\hline
\end{tabular}

20. SELF-DISCIPLINE (to restrain oneself, to resist temptation) is ...

\begin{tabular}{|c|c|c|c|c|c|c|c|c|}
\hline & 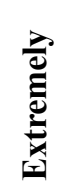 & $\stackrel{\mathscr{\Xi}}{\Xi}$ & 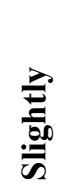 & 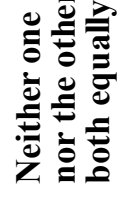 & 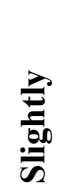 & $\stackrel{\mathscr{\Xi}}{0}$ & 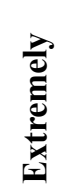 & \\
\hline $\begin{array}{l}\text {... important to } \\
\text { me }\end{array}$ & 0 & 0 & 0 & 0 & 0 & 0 & 0 & $\begin{array}{l}\text {... unimportant } \\
\text { to me }\end{array}$ \\
\hline $\begin{array}{l}\text {... something that } \\
\text { I am expected to } \\
\text { strive for in my } \\
\text { society }\end{array}$ & 0 & 0 & 0 & 0 & 0 & 0 & 0 & $\begin{array}{l}\text {... Something } \\
\text { that is my free } \\
\text { choice to strive } \\
\text { for in my society }\end{array}$ \\
\hline $\begin{array}{l}\text {... a moral value } \\
\text { to me }\end{array}$ & 0 & 0 & 0 & 0 & 0 & 0 & 0 & $\begin{array}{l}\text {... an immoral } \\
\text { value to me }\end{array}$ \\
\hline $\begin{array}{l}\text {... something bad } \\
\text { to follow }\end{array}$ & 0 & 0 & 0 & 0 & 0 & 0 & 0 & $\begin{array}{l}\text {... something } \\
\text { good to follow }\end{array}$ \\
\hline $\begin{array}{l}\text {... the right thing } \\
\text { to strive for }\end{array}$ & 0 & 0 & 0 & 0 & 0 & 0 & 0 & $\begin{array}{l}\ldots \text { the wrong } \\
\text { thing to strive for }\end{array}$ \\
\hline
\end{tabular}


21. SOCIAL JUSTICE (to correct injustice, to care for the weak) is ...

\begin{tabular}{|c|c|c|c|c|c|c|c|c|}
\hline & 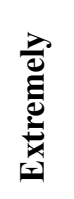 & $\stackrel{\mathscr{\Xi}}{\ddot{\sigma}}$ & 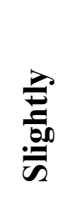 & 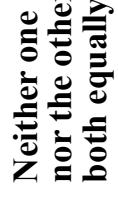 & 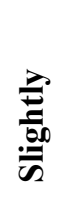 & $\stackrel{\mathscr{E}}{\ddot{\theta}}$ & 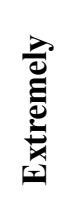 & \\
\hline $\begin{array}{l}\text { me important to } \\
\text { me }\end{array}$ & 0 & 0 & 0 & 0 & 0 & 0 & 0 & $\begin{array}{l}\text {... unimportant } \\
\text { to me }\end{array}$ \\
\hline $\begin{array}{l}\text {... something that } \\
\text { I am expected to } \\
\text { strive for in my } \\
\text { society }\end{array}$ & 0 & 0 & 0 & 0 & 0 & 0 & 0 & $\begin{array}{l}\text {... something } \\
\text { that is my free } \\
\text { choice to strive } \\
\text { for in my society }\end{array}$ \\
\hline $\begin{array}{l}\text {... a moral value } \\
\text { to me }\end{array}$ & 0 & 0 & 0 & 0 & 0 & 0 & 0 & $\begin{array}{l}\ldots \text { an immoral } \\
\text { value to me }\end{array}$ \\
\hline $\begin{array}{l}\text {... something bad } \\
\text { to follow }\end{array}$ & 0 & 0 & 0 & 0 & 0 & 0 & 0 & $\begin{array}{l}\text {... something } \\
\text { good to follow }\end{array}$ \\
\hline $\begin{array}{l}\text {.. the right thing } \\
\text { to strive for }\end{array}$ & 0 & 0 & 0 & 0 & 0 & 0 & 0 & $\begin{array}{l}\ldots \text { the wrong } \\
\text { thing to strive for }\end{array}$ \\
\hline
\end{tabular}

22. To be SUCCESSFUL (to achieve goals) is ...

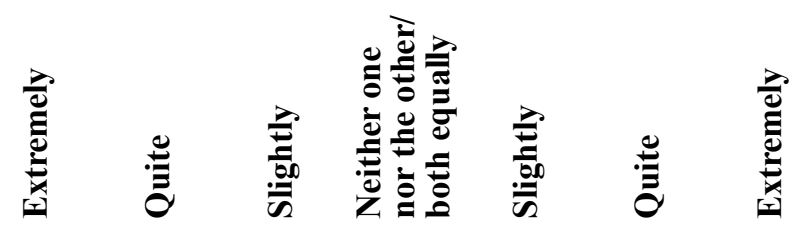

\begin{tabular}{|c|c|c|c|c|c|c|c|c|}
\hline $\begin{array}{l}\text { me important to } \\
\text { me }\end{array}$ & 0 & 0 & 0 & 0 & 0 & 0 & 0 & $\begin{array}{l}\text {... unimportant } \\
\text { to me }\end{array}$ \\
\hline $\begin{array}{l}\text {... something that } \\
\text { I am expected to } \\
\text { strive for in my } \\
\text { society }\end{array}$ & 0 & 0 & 0 & 0 & 0 & 0 & 0 & $\begin{array}{l}\text {... Something } \\
\text { that is my free } \\
\text { choice to strive } \\
\text { for in my society }\end{array}$ \\
\hline $\begin{array}{l}\text {... a moral value } \\
\text { to me }\end{array}$ & 0 & 0 & 0 & 0 & 0 & 0 & 0 & $\begin{array}{l}\ldots \text { an immoral } \\
\text { value to me }\end{array}$ \\
\hline $\begin{array}{l}\ldots \text { something bad } \\
\text { to follow }\end{array}$ & 0 & 0 & 0 & 0 & 0 & 0 & 0 & $\begin{array}{l}\text {... something } \\
\text { good to follow }\end{array}$ \\
\hline $\begin{array}{l}\ldots \text { the right thing } \\
\text { to strive for }\end{array}$ & 0 & 0 & 0 & 0 & 0 & 0 & 0 & $\begin{array}{l}\ldots \text { the wrong } \\
\text { thing to strive for }\end{array}$ \\
\hline
\end{tabular}


23. WEALTH (to emphasize material possessions, money etc.) is ...

\begin{tabular}{|c|c|c|c|c|c|c|c|c|}
\hline & 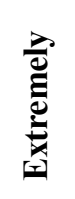 & $\stackrel{\mathscr{\Xi}}{0}$ & 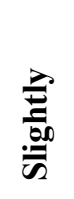 & 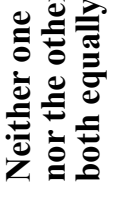 & 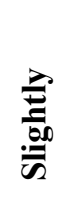 & $\stackrel{\mathscr{\Xi}}{\Xi}$ & 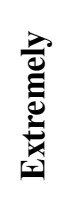 & \\
\hline $\begin{array}{l}\text { me important to } \\
\text { me }\end{array}$ & 0 & 0 & 0 & 0 & 0 & 0 & 0 & $\begin{array}{l}\text {... unimportant } \\
\text { to me }\end{array}$ \\
\hline $\begin{array}{l}\text {... something that } \\
\text { I am expected to } \\
\text { strive for in my } \\
\text { society }\end{array}$ & 0 & 0 & 0 & 0 & 0 & 0 & 0 & $\begin{array}{l}\text {... something } \\
\text { that is my free } \\
\text { choice to strive } \\
\text { for in my society }\end{array}$ \\
\hline $\begin{array}{l}\text {... a moral value } \\
\text { to me }\end{array}$ & 0 & 0 & 0 & 0 & 0 & 0 & 0 & $\begin{array}{l}\text {... an immoral } \\
\text { value to me }\end{array}$ \\
\hline $\begin{array}{l}\text {... something bad } \\
\text { to follow }\end{array}$ & 0 & 0 & 0 & 0 & 0 & 0 & 0 & $\begin{array}{l}\text {... something } \\
\text { good to follow }\end{array}$ \\
\hline $\begin{array}{l}\ldots \text { the right thing } \\
\text { to strive for }\end{array}$ & 0 & 0 & 0 & 0 & 0 & 0 & 0 & $\begin{array}{l}\ldots \text { the wrong } \\
\text { thing to strive for }\end{array}$ \\
\hline
\end{tabular}


What is your opinion regarding the following issues?

In the following section you will be presented some acts that some people may regard as wrong to do. We would like to know how wrong YOU think these acts are.

Please cross the appropriate bubble to indicate to which degree you think the following acts are wrong. The higher the number you use, the more wrong you judge this act; use in-between numbers for in-between wrongness.

\begin{tabular}{|c|c|c|c|c|c|}
\hline & 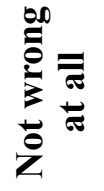 & 竞 & 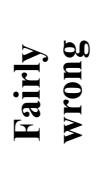 & $\sum^{2}$ & 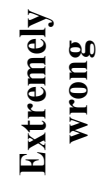 \\
\hline & 1 & 2 & 3 & 4 & 5 \\
\hline $\begin{array}{l}\text { 1. Claiming state benefits that you are not } \\
\text { entitled to }\end{array}$ & O & O & O & 0 & O \\
\hline 2. Avoiding a fare on public transport & O & O & O & O & $\mathrm{O}$ \\
\hline 3. Cheating on tax if you have the chance & 0 & 0 & 0 & O & 0 \\
\hline $\begin{array}{l}\text { 4. Someone accepting a bribe in the course of } \\
\text { their duties }\end{array}$ & O & O & O & O & O \\
\hline 5. Prostitution & O & O & O & O & O \\
\hline 6. Abortion & O & O & O & O & $\mathrm{O}$ \\
\hline 7. Divorce & 0 & O & 0 & O & O \\
\hline $\begin{array}{l}\text { 8. Euthanasia (terminating the life of the } \\
\text { incurable sick) }\end{array}$ & O & O & O & 0 & 0 \\
\hline 9. Suicide & 0 & 0 & 0 & 0 & 0 \\
\hline
\end{tabular}


Finally, we would like to ask you some questions about yourself. Please remember that you will never be personally identified in this research project or in any presentation or publication. The information you give, will be used for statistical purposes only.

Please answer the following question by ticking the appropriate box or writing the answer next to the question.

Gender? $\square$ Female $\square$ Male

What year were you born in?

What is your major?

To which country do you think you belong the most (whether by virtue of citizenship, length of residence, acculturation)?

In general, when it comes to politics, do you usually think of yourself as ...

\begin{tabular}{|c|c|c|c|c|c|c|}
\hline $\begin{array}{c}\text { Extremely } \\
\text { liberal } \\
\square\end{array}$ & $\begin{array}{c}\text { Liberal } \\
\square\end{array}$ & $\begin{array}{c}\text { Slightly } \\
\text { liberal } \\
\square\end{array}$ & $\begin{array}{c}\text { Moderate } \\
\square\end{array}$ & $\begin{array}{c}\text { Slightly } \\
\text { conservative } \\
\square\end{array}$ & $\begin{array}{c}\text { Conservative } \\
\square\end{array}$ & $\begin{array}{c}\text { Extremely } \\
\text { conservati } \\
\text { ve } \\
\square\end{array}$ \\
\hline
\end{tabular}

How important is religion to you?

$\begin{array}{llllllll}\text { Not at all important } & 1 & 2 & 3 & 4 & 5 & \text { Very important }\end{array}$

Thank you for participating. 
APPENDIX D: Value type rankings under five response scales in four individualistic and four collectivistic samples (Version 1)

\begin{tabular}{|c|c|c|c|c|c|c|c|c|c|c|}
\hline \multirow[b]{3}{*}{ Value type } & \multicolumn{10}{|c|}{ 'Important vs. Unimportant' Response Scale } \\
\hline & \multirow{2}{*}{$\begin{array}{l}\text { Pan- } \\
\text { cultural } \\
\text { sample }\end{array}$} & \multicolumn{4}{|c|}{ Individualistic samples } & \multicolumn{4}{|c|}{ Collectivistic samples } & \multirow{2}{*}{$\begin{array}{l}\text { Schwartz \& } \\
\text { Bardi (2001) }\end{array}$} \\
\hline & & Finland & Germany & U.K. & New Zealand & Brazil & Japan & The Philippines & Turkey & \\
\hline $\mathrm{BE}$ & 1 & 1 & 1 & 1 & 2 & 1 & 2 & 1 & 2 & 1 \\
\hline SD & 2 & 2 & 2 & 2 & 1 & 2 & 1 & 3 & 1 & 2 \\
\hline UN & 3 & 3 & 3 & 4 & 3 & 3 & 4 & 5 & 3 & 3 \\
\hline $\mathrm{AC}$ & 4 & 4 & 4 & 3 & 4 & 7 & 6 & 4 & 7 & 4 \\
\hline $\mathrm{CO}$ & 5 & 6 & 6 & 7 & 7 & 4 & 3 & 2 & 5 & 6 \\
\hline $\mathrm{HE}$ & 6 & 5 & 5 & 5 & 5 & 6 & 5 & 8 & 6 & 7 \\
\hline $\mathrm{SE}$ & 7 & 7 & 7 & 6 & 8 & 5 & 7 & 6 & 4 & 5 \\
\hline ST & 8 & 8 & 8 & 8 & 6 & 8 & 8 & 7 & 9 & 8 \\
\hline PO & 9 & 10 & 10 & 9 & 9 & 9 & 10 & 10 & 8 & 10 \\
\hline \multirow[t]{2}{*}{ TR } & 10 & 9 & 9 & 10 & 10 & 10 & 9 & 9 & 10 & 9 \\
\hline & \multicolumn{10}{|c|}{ 'Moral vs. Immoral' Response Scale } \\
\hline $\mathrm{BE}$ & 1 & 1 & 1 & 1 & 1 & 1 & 1 & 1 & 1 & 1 \\
\hline UN & 2 & 2 & 2 & 3 & 2 & 4 & 2 & 3 & 2.5 & 3 \\
\hline $\mathrm{CO}$ & 3 & 3 & 3 & 5 & 4 & 2 & 3 & 2 & 5 & 6 \\
\hline SD & 4 & 4 & 4 & 2 & 3 & 3 & 4 & 4 & 4 & 2 \\
\hline $\mathrm{SE}$ & 5 & 5 & 5 & 6 & 6 & 5 & 5 & 6 & 2.5 & 5 \\
\hline $\mathrm{AC}$ & 6 & 6 & 6 & 4 & 5 & 6 & 6 & 5 & 6 & 4 \\
\hline $\mathrm{TR}$ & 7 & 8 & 7 & 10 & 9 & 8 & 7 & 7 & 8 & 9 \\
\hline $\mathrm{HE}$ & 8 & 7 & 9 & 7 & 8 & 7 & 9 & 10 & 10 & 7 \\
\hline ST & 9 & 9 & 8 & 8 & 7 & 9 & 8 & 8 & 7 & 8 \\
\hline $\mathrm{PO}$ & 10 & 10 & 10 & 9 & 10 & 10 & 10 & 9 & 9 & 10 \\
\hline
\end{tabular}


'Expectation vs. Free choice' Response Scale

Individualistic samples $\quad$ Collectivistic samples

\begin{tabular}{|c|c|c|c|c|c|c|c|c|c|c|}
\hline Value type & $\begin{array}{l}\text { Pan-cultural } \\
\text { sample }\end{array}$ & Finland & Germany & U.K. & New Zealand & Brazil & Japan & The Philippines & Turkey & $\begin{array}{l}\text { Schwartz \& } \\
\text { Bardi (2001) } \\
\end{array}$ \\
\hline $\mathrm{AC}$ & 1 & 1 & 1 & 1 & 1 & 2 & 3 & 4 & 4 & 4 \\
\hline $\mathrm{CO}$ & 2 & 4 & 4.5 & 6 & 6 & 3 & 1 & 1 & 2 & 6 \\
\hline $\mathrm{SE}$ & 3 & 6 & 2 & 7 & 5 & 1 & 4 & 3 & 1 & 5 \\
\hline $\mathrm{BE}$ & 4 & 3 & 4.5 & 2 & 4 & 6 & 5 & 2 & 3 & 1 \\
\hline SD & 5 & 2 & 6 & 4 & 2 & 5 & 2 & 7 & 6 & 2 \\
\hline UN & 6 & 5 & 7.5 & 3 & 3 & 7 & 6 & 5 & 5 & 3 \\
\hline PO & 7 & 7 & 3 & 5 & 7 & 4 & 7 & 8 & 7 & 10 \\
\hline TR & 8 & 8 & 7.5 & 9 & 9 & 9 & 8 & 6 & 8 & 9 \\
\hline $\mathrm{HE}$ & 9 & 9 & 9 & 8 & 8 & 8 & 10 & 10 & 10 & 7 \\
\hline \multirow[t]{2}{*}{ ST } & 10 & 10 & 10 & 10 & 10 & 10 & 9 & 9 & 9 & 8 \\
\hline & \multicolumn{10}{|c|}{ 'Right vs. Wrong' Response Scale } \\
\hline $\mathrm{BE}$ & 1 & 1 & 1 & 1 & 1 & 1 & 1 & 1 & 1 & 1 \\
\hline UN & 2 & 2 & 2 & 3 & 2 & 3 & 3 & 4 & 3 & 3 \\
\hline SD & 3 & 3 & 3 & 4 & 4 & 4 & 2 & 5 & 5 & 2 \\
\hline $\mathrm{CO}$ & 4 & 4 & 4 & 5 & 6 & 2 & 4 & 2 & 4 & 6 \\
\hline $\mathrm{SE}$ & 5 & 5 & 7 & 6 & 5 & 5 & 5 & 6 & 2 & 5 \\
\hline $\mathrm{AC}$ & 6 & 6 & 5 & 2 & 3 & 6 & 6 & 3 & 6 & 4 \\
\hline ST & 7 & 8 & 8 & 8 & 7 & 8 & 7 & 8 & 8 & 8 \\
\hline $\mathrm{HE}$ & 8 & 7 & 6 & 7 & 8 & 7 & 8 & 10 & 9 & 7 \\
\hline TR & 9 & 9 & 9 & 9 & 9 & 10 & 9 & 7 & 10 & 9 \\
\hline $\mathrm{PO}$ & 10 & 10 & 10 & 10 & 10 & 9 & 10 & 9 & 7 & 10 \\
\hline
\end{tabular}


'Good vs. Bad' Response Scale

Individualistic samples

Collectivistic samples

\begin{tabular}{|c|c|c|c|c|c|c|c|c|c|c|}
\hline Value type & $\begin{array}{l}\text { Pan-cultural } \\
\text { sample }\end{array}$ & Finland & Germany & U.K. & New Zealand & Brazil & Japan & The Philippines & Turkey & $\begin{array}{l}\text { Schwartz \& } \\
\text { Bardi }(2001)\end{array}$ \\
\hline $\mathrm{BE}$ & 1 & 1 & 1 & 1 & 1 & 1 & 1 & 1 & 1 & 1 \\
\hline UN & 2 & 2 & 2 & 2 & 2 & 3 & 2 & 4 & 4 & 3 \\
\hline SD & 3 & 3 & 3 & 5 & 4 & 2 & 3 & 5 & 3 & 2 \\
\hline $\mathrm{CO}$ & 4 & 4 & 5 & 6 & 6 & 5 & 5 & 2 & 5 & 6 \\
\hline $\mathrm{AC}$ & 5 & 6 & 4 & 3 & 3 & 6 & 4 & 3 & 6 & 4 \\
\hline SE & 6 & 5 & 7 & 4 & 5 & 4 & 6 & 6 & 2 & 5 \\
\hline ST & 7 & 7 & 8 & 7 & 7 & 8 & 7 & 7 & 7 & 8 \\
\hline HE & 8 & 8 & 6 & 8 & 8 & 7 & 8 & 10 & 9 & 7 \\
\hline TR & 9 & 9 & 9 & 9 & 9 & 10 & 9 & 8 & 10 & 9 \\
\hline PO & 10 & 10 & 10 & 10 & 10 & 9 & 10 & 9 & 8 & 10 \\
\hline
\end{tabular}

Note. Rank orders are based on survey version one. Rank orders for survey version two were very similar (see MDS analysis). UN $=$ Universalism, BE $=$ Benevolence, TR

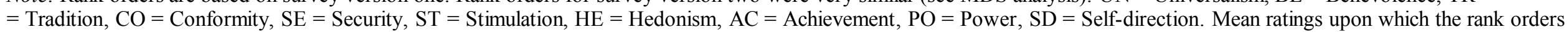
are based as well as standard deviations can be obtained from the author. 
APPENDIX E: Comparison of MDS representations based on rankings (left figure) and mean ratings (right figure) after transformation to maximal similarity with GPA
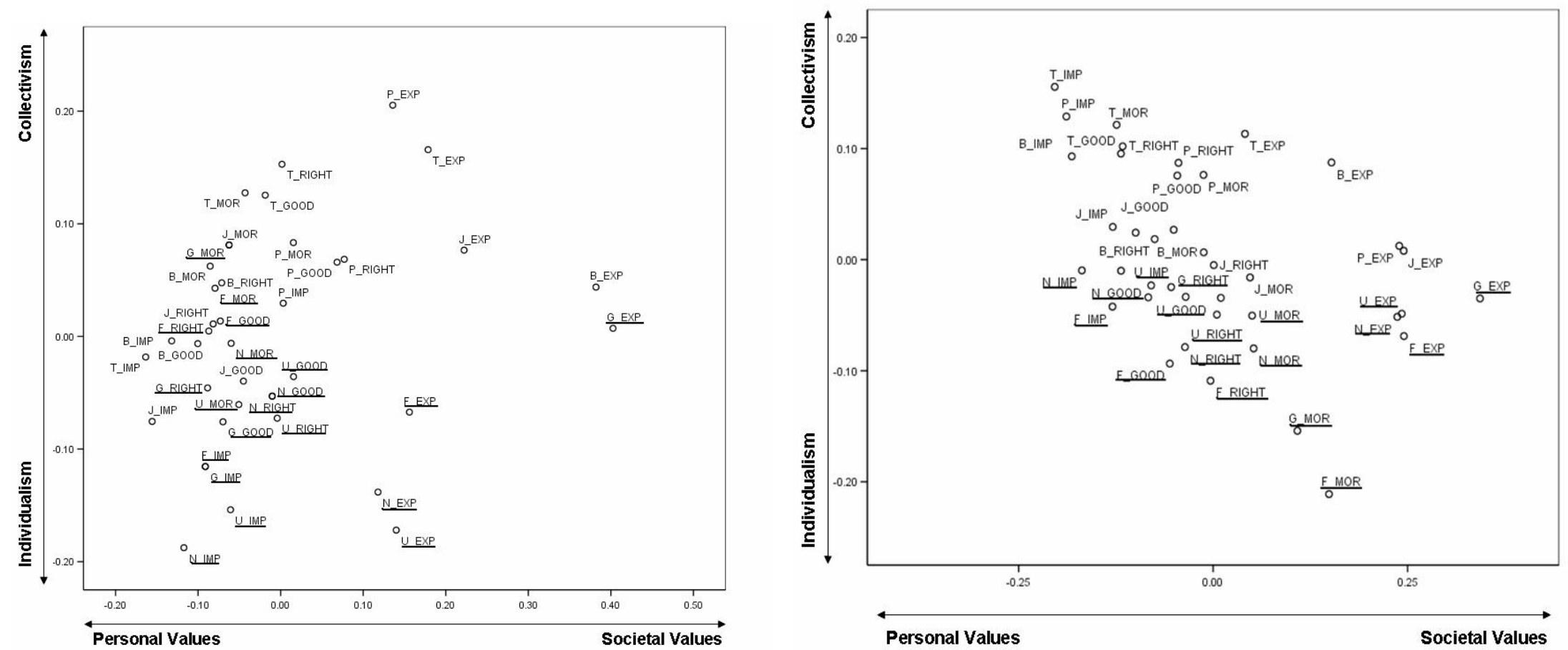

Note. Underlined objects denote individualistic cultural samples according to the literature. $\mathrm{B}=$ Brazil, $\mathrm{F}=$ Finland, $\mathrm{G}=$ Germany, $\mathrm{J}=\mathrm{Japan}, \mathrm{N}=\mathrm{New}$ Zealand, $\mathrm{P}=$ the Philippines, $\mathrm{T}=$ Turkey, $\mathrm{U}=$ United Kingdom, $\mathrm{EXP}=$ 'expectation vs. free choice' response scale, MOR = 'moral vs immoral' response scale, RIGHT = right vs. wrong' response scale, GOOD $=$ 'good vs. bad' response scale, IMP = 'important vs. unimportant' response scale. 
APPENDIX F: Example of free-listed moral traits categories (Filipino responses)

\begin{tabular}{lll}
\hline & Moral trait & Original Responses \\
\hline 1 & accommodating & accomodating \\
\hline 3 & angel & angel \\
\hline 4 & approachable & approachable \\
\hline 5 & balanced & balanced \\
\hline 6 & brave & benevolant \\
\hline 7 & caring & brave/ confident \\
& & caring \\
& & has care for others \\
\hline 8 & catholic & cares for others \\
\hline 9 & charismatic & charismatic based beliefs \\
\hline 10 & christian & $\begin{array}{l}\text { christian } \\
\text { christian doctrine }\end{array}$ \\
\hline 11 & clean & clean \\
& & clean in thought, word and deed \\
\hline 12 & compassionate & $\begin{array}{l}\text { c compassionate person } \\
\text { compassion }\end{array}$ \\
\hline 13 & confident & confident \\
& & brave/ confident \\
\hline 14 & conscientious & conscience \\
& & listens to conscience \\
\hline 15 & conscious & conscious \\
\hline 16 & considerate & considerate \\
\hline
\end{tabular}

someone who thinks of other people's feelings

a person sensitive to the need of others

thinks of other opinions to be considered when making decisions

sensitive to other people

\begin{tabular}{lll}
\hline 17 & controlled & $\begin{array}{l}\text { controlled } \\
\text { self-contro }\end{array}$ \\
\hline 18 & correct & correct \\
\hline 19 & courageous & courageou \\
\hline 20 & courteous & courteous
\end{tabular}

courtesy

attributes good virtues (courteous, truthful)

\begin{tabular}{lll}
\hline 21 & critical & someone who thinks critically \\
\hline 22 & decent & $\begin{array}{l}\text { decent } \\
\text { descent }\end{array}$ \\
\hline 23 & decided & decided
\end{tabular}

makes clear cut decisions

makes decisions not only for herself but also for others

\begin{tabular}{lll}
\hline 24 & democratic & democratic \\
\hline 25 & dependable & dependable \\
\hline 26 & dignified & dignified \\
\hline 27 & disciplined & $\begin{array}{l}\text { disciplined } \\
\text { discipline }\end{array}$ \\
& & well-discipline
\end{tabular}

\begin{tabular}{lll}
\hline 28 & divine & divine \\
\hline 29 & educated & educated \\
\hline 30 & enthusiastic & enthusiastic \\
\hline 31 & ethical & ethical \\
& & someone who is ethical \\
& & ethics \\
\hline 32 & exemplary & role model \\
& & sets a good example for himself and others \\
& & an example \\
\hline
\end{tabular}




\begin{tabular}{|c|c|c|}
\hline & & $\begin{array}{l}\text { good-model } \\
\text { idol } \\
\text { model citizen } \\
\text { model } \\
\text { teacher } \\
\end{array}$ \\
\hline & fair & $\begin{array}{l}\text { fair } \\
\text { someone just and fair }\end{array}$ \\
\hline & faithful & $\begin{array}{l}\text { faithful } \\
\text { faith } \\
\text { someone who believes in faith... }\end{array}$ \\
\hline & firm & firm principles \\
\hline 36 & flexible & flexible \\
\hline & follows rules & $\begin{array}{l}\text { law-abiding } \\
\text { does not disobey laws and rules } \\
\text { follows rules and regulations } \\
\text { follows the norms/ laws of the church } \\
\text { follows the rules } \\
\text { someone who implements the rules }\end{array}$ \\
\hline 38 & forgiving & forgiving \\
\hline 39 & friend & friends \\
\hline 40 & friendly & friendly \\
\hline & generous & $\begin{array}{l}\text { generous } \\
\text { generosity } \\
\text { giving/generous }\end{array}$ \\
\hline & gentle & $\begin{array}{l}\text { gentle } \\
\text { gentle to others } \\
\text { one who is gentle }\end{array}$ \\
\hline 43 & genuine & genuine \\
\hline 44 & giving & giving/ generous \\
\hline & god & $\begin{array}{l}\text { God } \\
\text { God like } \\
\text { Godly }\end{array}$ \\
\hline & god-fearing & $\begin{array}{l}\text { God-fearing } \\
\text { fear of God }\end{array}$ \\
\hline & good & $\begin{array}{l}\text { good } \\
\text { good person } \\
\text { good conduct } \\
\text { good \& evil } \\
\text { one who does good things } \\
\text { has a good disposition } \\
\text { good attitude } \\
\text { with good ethics } \\
\text { good behavior } \\
\text { gives something good to the country } \\
\text { good or bad } \\
\text { good personality } \\
\text { believes in good values } \\
\text { performs good manners } \\
\text { good standing } \\
\text { good will } \\
\text { good practice } \\
\text { good and bad } \\
\text { good breeding } \\
\text { good upbringing } \\
\text { someone who is inherently good... } \\
\text { good trait } \\
\text { one who has good judgement } \\
\text { good citizen } \\
\text { does something good all the time } \\
\text { do gooder } \\
\text { attributes good virtues (courteous, truthful) }\end{array}$ \\
\hline
\end{tabular}




\begin{tabular}{|c|c|c|}
\hline & & $\begin{array}{l}\text { good behavior } \\
\text { has good breeding } \\
\text { good model to others }\end{array}$ \\
\hline 48 & grateful & is grateful for being able to live \\
\hline 49 & hard-working & hardworking \\
\hline 50 & has beliefs & beliefs \\
\hline 51 & has integrity & $\begin{array}{l}\text { integrity } \\
\text { with integrity } \\
\text { has integrity }\end{array}$ \\
\hline 52 & heart & heart \\
\hline 53 & helpful & $\begin{array}{l}\text { helps others (ex: those less fortunate) } \\
\text { a person willing to help others } \\
\text { always willing to help } \\
\text { helpful to others } \\
\text { helpful }\end{array}$ \\
\hline 54 & holy & holy \\
\hline 55 & honest & $\begin{array}{l}\text { honest } \\
\text { honesty } \\
\text { sense of honesty } \\
\text { does not cheat }\end{array}$ \\
\hline 56 & honourable & honorable \\
\hline 57 & humanistic & humanistic \\
\hline 58 & humble & $\begin{array}{l}\text { humble } \\
\text { humility } \\
\text { humbleness }\end{array}$ \\
\hline 59 & independent & independent \\
\hline 60 & influential & $\begin{array}{l}\text { influence of peers } \\
\text { influences }\end{array}$ \\
\hline 61 & innocent & innocence \\
\hline 62 & intangible & something intangible \\
\hline 63 & intelligent & intelligent \\
\hline 64 & just & $\begin{array}{l}\text { just } \\
\text { always does what is right and just } \\
\text { advocates social justice } \\
\text { great sense of justice } \\
\text { have a great sense of justice and righteousness } \\
\text { someone just and fair } \\
\text { fights for justice } \\
\text { unjust }\end{array}$ \\
\hline 65 & kind & $\begin{array}{l}\text { kind } \\
\text { kind-hearted } \\
\text { kindly guy } \\
\text { one who is kind } \\
\text { kindness }\end{array}$ \\
\hline 66 & knowledgeable & knowledgable \\
\hline 67 & leader & leadership \\
\hline 68 & learner & learner \\
\hline 69 & logical & someone who thinks logically... \\
\hline 70 & loving & $\begin{array}{l}\text { loving } \\
\text { love } \\
\text { loving other people } \\
\text { love god } \\
\end{array}$ \\
\hline 71 & loyal & loyal \\
\hline 72 & mature & mature \\
\hline 73 & mindful & mindful \\
\hline 74 & moral & $\begin{array}{l}\text { moral } \\
\text { morality } \\
\text { how one views morality } \\
\text { has good morals }\end{array}$ \\
\hline
\end{tabular}




\begin{tabular}{|c|c|c|}
\hline 75 & nationalist & nationalist \\
\hline 76 & nice & $\begin{array}{l}\text { nice } \\
\text { nice personality }\end{array}$ \\
\hline 77 & not harming & $\begin{array}{l}\text { doesn't cause any harm/ damage } \\
\text { does not intentionally harm others } \\
\text { someone who doesn't do anything bad to others } \\
\text { against harm }\end{array}$ \\
\hline 78 & obedient & $\begin{array}{l}\text { obedient } \\
\text { obedient and can be respected }\end{array}$ \\
\hline 79 & objective & objectivity \\
\hline 80 & open & open-minded \\
\hline 81 & optimistic & $\begin{array}{l}\text { optimism } \\
\text { optimistic }\end{array}$ \\
\hline 82 & outgoing & outgoing \\
\hline 83 & patient & patient \\
\hline 84 & peaceful & $\begin{array}{l}\text { inner peace } \\
\text { aims for peace and equality in the community }\end{array}$ \\
\hline 85 & perfect & almost perfect $=\mathrm{P}$ \\
\hline 86 & perseverant & perseverant \\
\hline 87 & pious & pious \\
\hline 88 & polite & polite \\
\hline 89 & positive & $\begin{array}{l}\text { positive } \\
\text { positive thinker }\end{array}$ \\
\hline 90 & post-conventional & at the post conventional level of moral development \\
\hline 91 & principled & $\begin{array}{l}\text { principled } \\
\text { have principles } \\
\text { has principles } \\
\text { principle } \\
\text { someone with principles and values }\end{array}$ \\
\hline 92 & promising & promising \\
\hline 93 & proper & $\begin{array}{l}\text { Proper } \\
\text { prim and proper }\end{array}$ \\
\hline 94 & quiet & quiet-type \\
\hline 95 & rational & someone who is rational... \\
\hline 96 & relaxed & relaxed \\
\hline 97 & religious & $\begin{array}{l}\text { religious } \\
\text { religiousness } \\
\text { religiously wise } \\
\text { religion } \\
\text { someone who is religious... } \\
\text { religious/ has strong belief } \\
\text { religious term } \\
\text { respects the God of his religion } \\
\text { practices religion consciensciously } \\
\text { has a firm faith in his/ her own religion } \\
\text { lives according to God's will } \\
\text { someone who prays } \\
\text { knows how to pray }\end{array}$ \\
\hline 98 & resilient & resilient \\
\hline 99 & respectable & respectable \\
\hline 100 & respected & $\begin{array}{l}\text { obedient and can be respected } \\
\text { respectful/ respected }\end{array}$ \\
\hline 101 & respectful & $\begin{array}{l}\text { respects the God of his religion } \\
\text { respectful/ respected } \\
\text { respects authority except when unjust } \\
\text { one who is respectful } \\
\text { respects his/ her family } \\
\text { respect } \\
\text { has respect }\end{array}$ \\
\hline
\end{tabular}




\begin{tabular}{|c|c|c|}
\hline & & $\begin{array}{l}\text { a respectful person } \\
\text { respects others and his/ herself } \\
\text { respect }\end{array}$ \\
\hline 102 & responsible & $\begin{array}{l}\text { responsible } \\
\text { responsible in ones' duties }\end{array}$ \\
\hline 103 & reverent & reverent \\
\hline 104 & right & $\begin{array}{l}\text { right } \\
\text { right and wrong } \\
\text { always does what is right and just } \\
\text { always what he know is right } \\
\text { a person who does what is right } \\
\text { always does the right thing } \\
\text { does what is right and not the wrong } \\
\text { the right thing to do } \\
\text { believes what he thinks is right } \\
\text { what is right } \\
\text { inclined to do what is right } \\
\text { follows what is right } \\
\text { does the right thing even under pressure } \\
\text { knows what is right from wrong } \\
\text { has the ability to distinguish right from wrong } \\
\text { knows what is right and wrong } \\
\text { has a sense of what is right and wrong } \\
\text { knows right from wrong } \\
\text { can distinguish what is right and wrong } \\
\text { knows what is wring and right } \\
\text { knows right from wrong }\end{array}$ \\
\hline 105 & righteous & $\begin{array}{l}\text { righteous } \\
\text { have a great sense of justice and righteousness }\end{array}$ \\
\hline 106 & saints & saints \\
\hline 107 & secure & a secure person \\
\hline 108 & self-determined & $\begin{array}{l}\text { has their own set of beliefs } \\
\text { looks at things in their own perspective }\end{array}$ \\
\hline 109 & sharing & sharing \\
\hline 110 & sincere & sincere \\
\hline 111 & sinless & close to being sinless/ free of $\sin$ \\
\hline 112 & smart & smart \\
\hline 113 & sociable & $\begin{array}{l}\text { social skills } \\
\text { social aspects of life } \\
\text { socially accepted }\end{array}$ \\
\hline 114 & soulfulness & soulfulness \\
\hline 115 & standing up & stands up for what he believes in \\
\hline 116 & strict & $\begin{array}{l}\text { strict (overpowering superego) } \\
\text { strict/ restrictions }\end{array}$ \\
\hline 117 & strong & strong principles \\
\hline 118 & successful & success \\
\hline 119 & suck up & a suck up \\
\hline 120 & sympathetic & $\begin{array}{l}\text { who has sympathy for the poor } \\
\text { someone who pities others easily }\end{array}$ \\
\hline 121 & tactful & tactful \\
\hline 122 & thoughtful & thoughtful \\
\hline 123 & tolerant & $\begin{array}{l}\text { tries his/ her best to be not discriminating against other genders and } \\
\text { ethnicities }\end{array}$ \\
\hline 124 & trust & $\begin{array}{l}\text { trust } \\
\text { trustworthy }\end{array}$ \\
\hline 125 & truthful & $\begin{array}{l}\text { truthful } \\
\text { attributes good virtues (courteous, truthful) }\end{array}$ \\
\hline 126 & uebermensch & uebermensch \\
\hline
\end{tabular}




\begin{tabular}{|c|c|c|}
\hline 127 & understanding & $\begin{array}{l}\text { understanding } \\
\text { understands other people's morals }\end{array}$ \\
\hline 128 & unselfish & $\begin{array}{l}\text { thinks of everyone's sake not only his } \\
\text { wellfare of others } \\
\text { service to others' } \\
\text { unselfish } \\
\text { sacrificing for the good of all } \\
\text { selfless }\end{array}$ \\
\hline 129 & upright & $\begin{array}{l}\text { upright } \\
\text { morally upright }\end{array}$ \\
\hline 130 & uptight & uptight \\
\hline 131 & values & $\begin{array}{l}\text { values } \\
\text { protects values } \\
\text { values human rights }\end{array}$ \\
\hline 132 & virtuous & $\begin{array}{l}\text { virtuous } \\
\text { virtue } \\
\text { virtues } \\
\text { has virtues } \\
\text { lives their life with those values/ virtues in mind }\end{array}$ \\
\hline 133 & well-balanced & well-balanced \\
\hline 134 & well-mannered & $\begin{array}{l}\text { well mannered } \\
\text { with manners } \\
\text { proper etiquette }\end{array}$ \\
\hline 135 & well-raised & $\begin{array}{l}\text { well-raised } \\
\text { upbringing }\end{array}$ \\
\hline 136 & well-rounded & well-rounded \\
\hline 137 & wise & $\begin{array}{l}\text { wise } \\
\text { religiously wise } \\
\text { wisdom }\end{array}$ \\
\hline 138 & yin yang & yin yang \\
\hline 139 & $\begin{array}{l}\text { [excluded from } \\
\text { analysis] }\end{array}$ & $\begin{array}{l}\text { pagkantas [meaning unclear] } \\
\text { attitude } \\
\text { attitudes } \\
\text { basis } \\
\text { behavior } \\
\text { characteristic } \\
\text { culture } \\
\text { emotions } \\
\text { environment } \\
\text { family } \\
\text { inbedded in culture } \\
\text { internal attributes of a person (emotional, social, etc.) } \\
\text { learned } \\
\text { mental processes } \\
\text { nature and nurture } \\
\text { part of society } \\
\text { person } \\
\text { personality } \\
\text { phd (doctorate) } \\
\text { point of view of society } \\
\text { sin } \\
\text { subjective, differs in every culture and society } \\
\text { temparent } \\
\text { the standing of a person in a group/ class } \\
\text { theology } \\
\text { traits } \\
\text { wish it ain't put in fundamentalism }\end{array}$ \\
\hline
\end{tabular}

Note. Responses given by one person are put into one single line. 
APPENDIX G: Biplots using correspondence analysis based on culturally idiosyncratic moral trait categories (left figure) and all 49 moral trait categories (right figure).
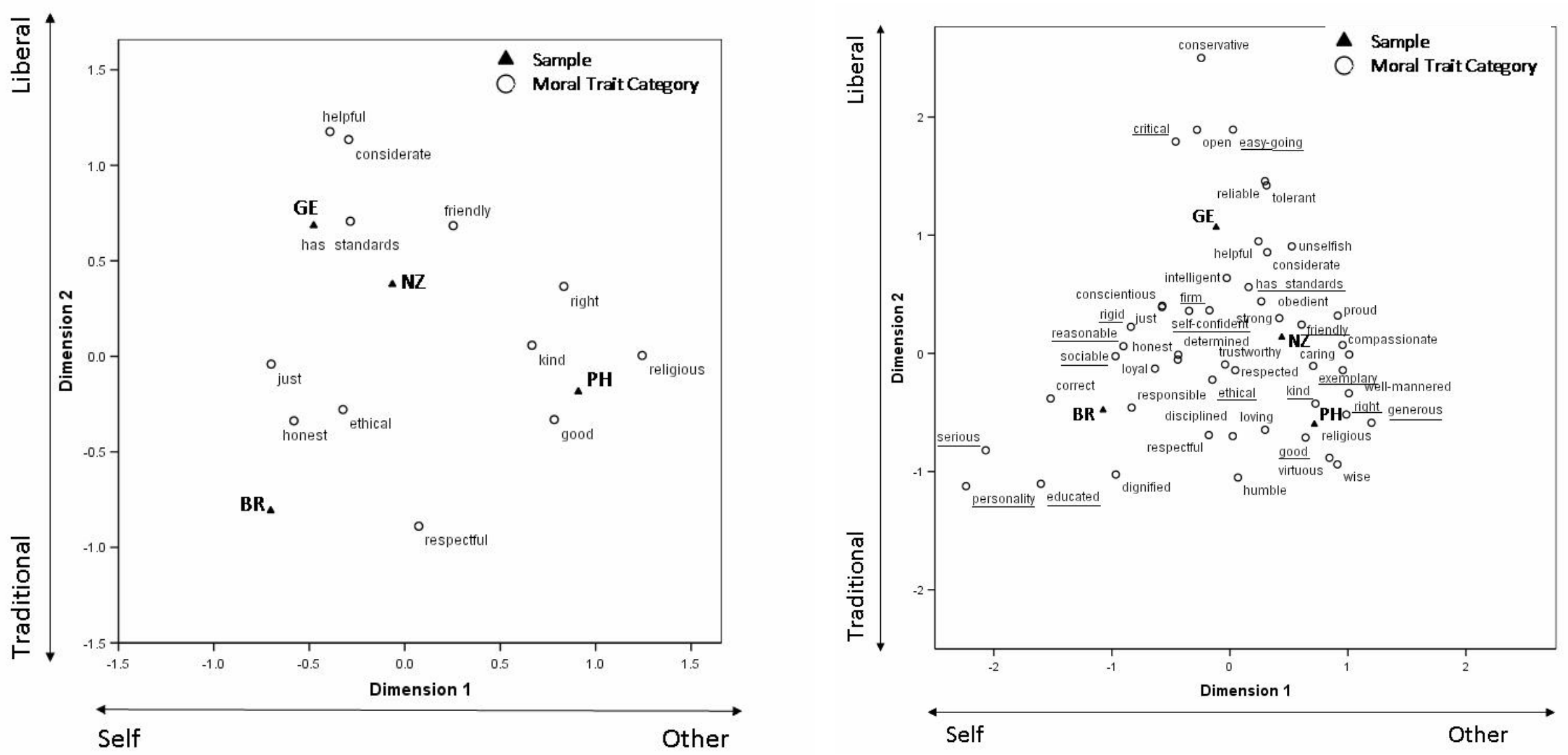

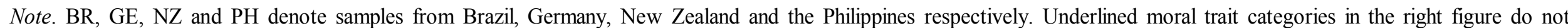
correspond to any of Schwartz' (1992) values from his survey. 
APPENDIX H: Moral trait categories matched to values of the Schwartz' Value Survey

\begin{tabular}{|c|c|c|}
\hline Moral Category & Schwartz' Value Survey & Value type \\
\hline $\begin{array}{l}\text { DETERMINED (ambitious, } \\
\text { decided, perseverant); } \\
\text { HARDWORKING }\end{array}$ & AMBITIOUS (hard-working, aspiring) & $\mathrm{AC}$ \\
\hline \multirow[t]{2}{*}{ LEADER (influential) } & $\begin{array}{l}\text { INFLUENTIAL (having an impact on people and } \\
\text { events) }\end{array}$ & $\mathrm{AC}$ \\
\hline & CAPABLE (competent, effective, efficient) & $\mathrm{AC}$ \\
\hline SUCCESSFUL & SUCCESSFUL (achieving goals) & $\mathrm{AC}$ \\
\hline LOYAL (solidary) & LOYAL (faithful to my friends, group) & $\mathrm{BE}$ \\
\hline $\begin{array}{l}\text { HONEST (authentic, genuine, } \\
\text { has integrity, sincere, } \\
\text { transparent, true, truthful) }\end{array}$ & HONEST (genuine, sincere) & $\mathrm{BE}$ \\
\hline HELPFUL (supportive) & HELPFUL (working for the welfare of others) & $\mathrm{BE}$ \\
\hline \multirow[t]{2}{*}{$\begin{array}{l}\text { RESPONSIBLE; RELIABLE } \\
\text { (dependable) }\end{array}$} & RESPONSIBLE (dependable, reliable) & $\mathrm{BE}$ \\
\hline & FORGIVING (willing to pardon others) & $\mathrm{BE}$ \\
\hline $\begin{array}{l}\text { WELL-MANNERED } \\
\text { (courteous, tactful, polite, well- } \\
\text { raised) }\end{array}$ & POLITENESS (courtesy, good manners) & $\mathrm{CO}$ \\
\hline DISCIPLINED (controlled) & $\begin{array}{l}\text { SELF-DISCIPLINE (self-restraint, resistance to } \\
\text { temptation) }\end{array}$ & $\mathrm{CO}$ \\
\hline RESPECTFUL & $\begin{array}{l}\text { HONOURING OF PARENTS AND ELDERS } \\
\text { (showing respect) }\end{array}$ & $\mathrm{CO}$ \\
\hline \multirow{5}{*}{$\begin{array}{l}\text { OBEDIENT (follows rules, not } \\
\text { rebellious) }\end{array}$} & OBEDIENT (dutiful, meeting obligations) & $\mathrm{CO}$ \\
\hline & PLEASURE (gratification of desires) & $\mathrm{HE}$ \\
\hline & $\begin{array}{l}\text { ENJOYING LIFE (enjoying food, sex, leisure, } \\
\text { etc.) }\end{array}$ & $\mathrm{HE}$ \\
\hline & SELF-INDULGENT (doing pleasant things) & $\mathrm{HE}$ \\
\hline & WEALTH (material possessions, money) & $\mathrm{PO}$ \\
\hline LEADER (influential) & AUTHORITY (the right to lead or command) & $\mathrm{PO}$ \\
\hline \multirow[t]{4}{*}{ CONTROLLING } & $\begin{array}{l}\text { SOCIAL POWER (control over others, } \\
\text { dominance) }\end{array}$ & $\mathrm{PO}$ \\
\hline & $\begin{array}{l}\text { PRESERVING MY PUBLIC IMAGE } \\
\text { (protecting my "face") }\end{array}$ & $\mathrm{PO}$ \\
\hline & $\begin{array}{l}\text { OBSERVING SOCIAL NORMS (to maintain } \\
\text { face) }\end{array}$ & $\mathrm{PO}$ \\
\hline & CREATIVITY (uniqueness, imagination) & SD \\
\hline \multirow[t]{2}{*}{ INDEPENDENT (self-determined) } & INDEPENDENT (self-reliant, self-sufficient) & SD \\
\hline & $\begin{array}{l}\text { CHOOSING OWN GOALS (selecting own } \\
\text { purposes) }\end{array}$ & SD \\
\hline LIBERAL & FREEDOM (freedom of action and thought) & SD \\
\hline \multirow[t]{4}{*}{ INTERESTED (curious) } & CURIOUS (interested in everything, exploring) & SD \\
\hline & $\begin{array}{l}\text { NATIONAL SECURITY (protection of my } \\
\text { nation from enemies) }\end{array}$ & SE \\
\hline & $\begin{array}{l}\text { RECIPROCATION OF FAVOURS (avoidance } \\
\text { of indebtedness) }\end{array}$ & SE \\
\hline & FAMILY SECURITY (safety for loved ones) & $\mathrm{SE}$ \\
\hline \multirow[t]{3}{*}{ CLEAN } & CLEAN (neat, tidy) & SE \\
\hline & SOCIAL ORDER (stability of society) & SE \\
\hline & $\begin{array}{l}\text { A VARIED LIFE (filled with challenge, novelty } \\
\text { and change) }\end{array}$ & ST \\
\hline \multirow[t]{2}{*}{ BRAVE (courageous) } & DARING (seeking adventure, risk) & ST \\
\hline & AN EXCITING LIFE (stimulating experiences) & ST \\
\hline CONSERVATIVE (conventional) & $\begin{array}{l}\text { RESPECT FOR TRADITION (preservation of } \\
\text { time-honoured customs) }\end{array}$ & TR \\
\hline
\end{tabular}




\begin{tabular}{|c|c|c|}
\hline Moral Category & Schwartz' Value Survey & Value type \\
\hline WELL-BALANCED (balanced) & $\begin{array}{l}\text { MODERATE (avoiding extremes of feeling \& } \\
\text { action) }\end{array}$ & TR \\
\hline \multirow[t]{2}{*}{ HUMBLE (modest) } & HUMBLE (modest, self-effacing) & TR \\
\hline & $\begin{array}{l}\text { ACCEPTING MY PORTION IN LIFE } \\
\text { (submitting to life's circumstances) }\end{array}$ & TR \\
\hline $\begin{array}{l}\text { RELIGIOUS (Christian, } \\
\text { faithful, god, god-fearing, holy, } \\
\text { pray) }\end{array}$ & DEVOUT (holding to religious faith \& belief) & TR \\
\hline $\begin{array}{l}\text { JUST (egalitarian, equal, fair, } \\
\text { impartial, righteous) }\end{array}$ & EQUALITY (equal opportunity for all) & UN \\
\hline \multirow[t]{2}{*}{ PEACEFUL } & $\begin{array}{l}\text { A WORLD AT PEACE (free of war and } \\
\text { conflict) }\end{array}$ & UN \\
\hline & UNITY WITH NATURE (fitting into nature) & UN \\
\hline \multirow[t]{2}{*}{ WISE; ENLIGHTENED } & WISDOM (a mature understanding of life) & UN \\
\hline & $\begin{array}{l}\text { A WORLD OF BEAUTY (beauty of nature and } \\
\text { the arts) }\end{array}$ & UN \\
\hline STANDING UP & $\begin{array}{l}\text { SOCIAL JUSTICE (correcting injustice, care for } \\
\text { the weak) }\end{array}$ & UN \\
\hline $\begin{array}{l}\text { OPEN; TOLERANT (non- } \\
\text { judgmental) }\end{array}$ & $\begin{array}{l}\text { BROADMINDED (tolerant of different ideas and } \\
\text { beliefs) }\end{array}$ & UN \\
\hline \multirow[t]{2}{*}{$\begin{array}{l}\text { ENVIRONMENTAL } \\
\text { AWARENESS }\end{array}$} & $\begin{array}{l}\text { PROTECTING THE ENVIRONMENT } \\
\text { (preserving nature) }\end{array}$ & UN \\
\hline & MEANING IN LIFE (a purpose in life) & $\mathrm{X}$ \\
\hline $\begin{array}{l}\text { PROUD (self-respect, high self- } \\
\text { regard) }\end{array}$ & SELF RESPECT (belief in one's own worth) & $\mathrm{X}$ \\
\hline \multirow[t]{3}{*}{ LOVING } & $\begin{array}{l}\text { MATURE LOVE (deep emotional \& spiritual } \\
\text { intimacy) }\end{array}$ & $\mathrm{X}$ \\
\hline & INNER HARMONY (at peace with myself) & $\mathrm{X}$ \\
\hline & PRIVACY ( the right to have a private sphere) & $\mathrm{X}$ \\
\hline $\begin{array}{l}\text { RESPECTED (admired, } \\
\text { honourable) }\end{array}$ & $\begin{array}{l}\text { SOCIAL RECOGNITION (respect, approval by } \\
\text { others) }\end{array}$ & $\mathrm{X}$ \\
\hline \multirow[t]{2}{*}{ FRIEND } & TRUE FRIENDSHIP (close, supportive friends) & $\mathrm{X}$ \\
\hline & $\begin{array}{l}\text { HEALTHY (not being sick physically or } \\
\text { mentally) }\end{array}$ & $\mathrm{X}$ \\
\hline \multirow[t]{3}{*}{ INTELLIGENT (smart) } & INTELLIGENT (logical, thinking) & $\mathrm{X}$ \\
\hline & $\begin{array}{l}\text { A SPIRITUAL LIFE (emphasis on spiritual not } \\
\text { material matters) }\end{array}$ & $\mathrm{X}$ \\
\hline & $\begin{array}{l}\text { SENSE OF BELONGING (feeling that others } \\
\text { care about me) }\end{array}$ & $\mathrm{X}$ \\
\hline \multicolumn{3}{|c|}{$\begin{array}{l}\text { Note. Moral trait categories were here also matched if their frequency did not exceed five percent in any } \\
\text { of the samples (see above explanation of the } 5 \% \text { judgment rule). Some moral trait categories were } \\
\text { matched to several values (see e.g. LEADER). Moral categories in boldface are central moral features in } \\
\text { at least one of the four cultures studied. Features in brackets are those that were allocated to the more } \\
\text { general moral trait category. The key for value types is: AC = Achievement, } \mathrm{BE}=\text { Benevolence, } \mathrm{CO}= \\
\text { Conformity, HE = Hedonism, PO = Power, } \mathrm{SD}=\mathrm{Self}-\text { direction, } \mathrm{SE}=\mathrm{Security}, \mathrm{ST}=\mathrm{Stimulation}, \mathrm{TR}= \\
\text { Tradition, UN = Universalism, } \mathrm{X}=\text { values with inequivalent meaning across cultures according to } \\
\text { Schwartz (1992). }\end{array}$} \\
\hline
\end{tabular}

